
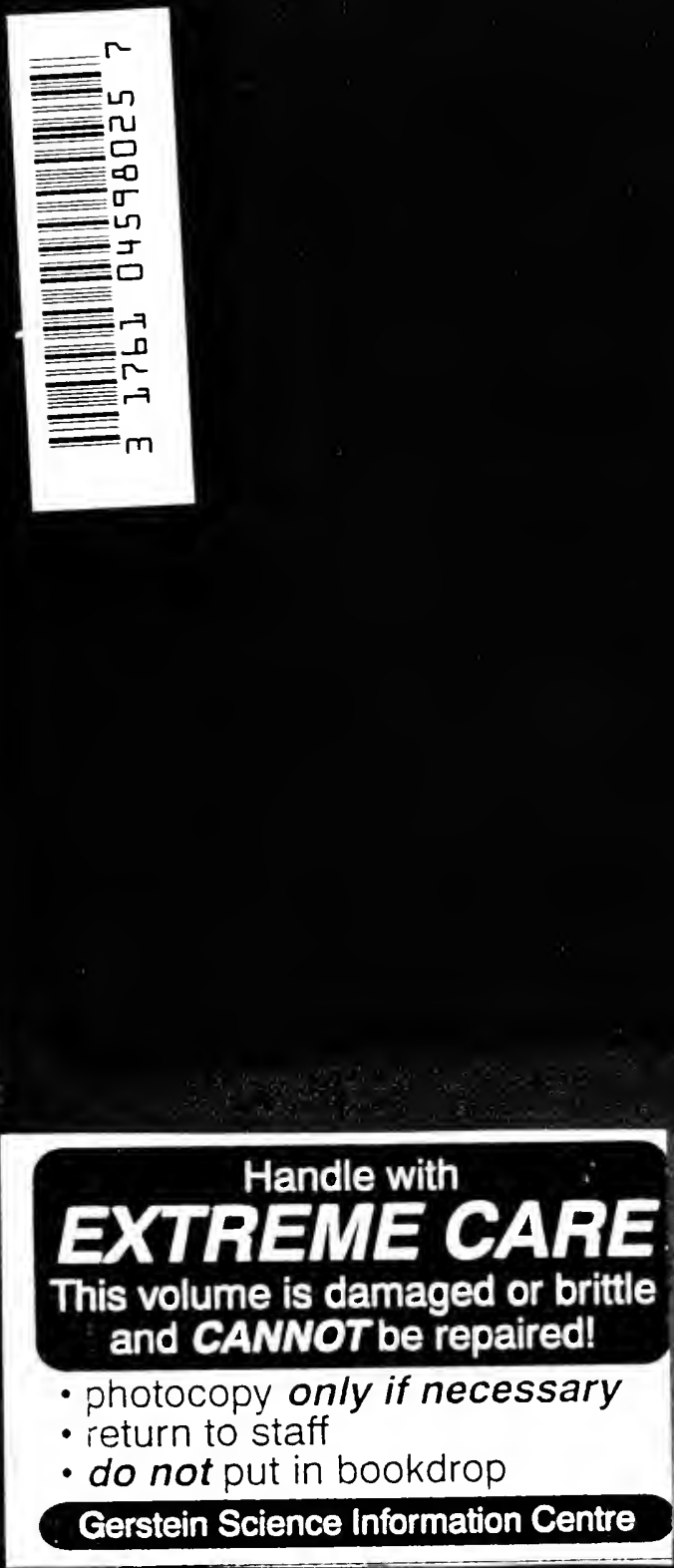
Digitized by the Internet Archive in 2007 with funding from Microsoft Corporation

http//www.archive.org/details/applicationsofdy00thomuoft 


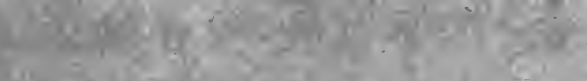

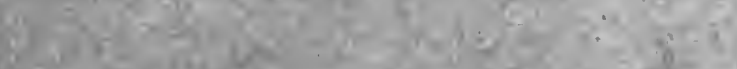

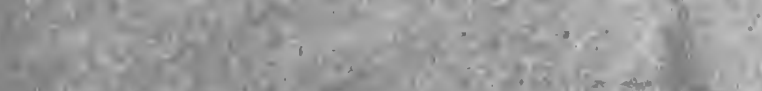

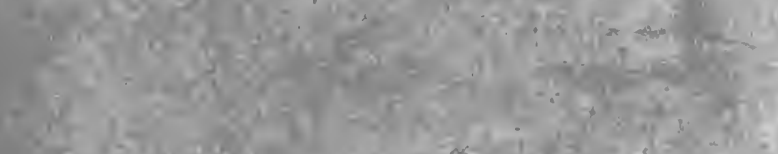

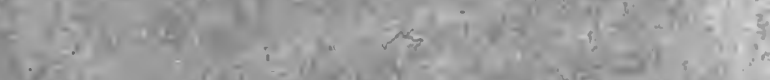

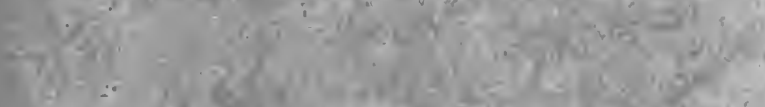
ged

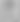

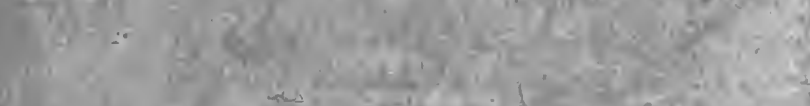

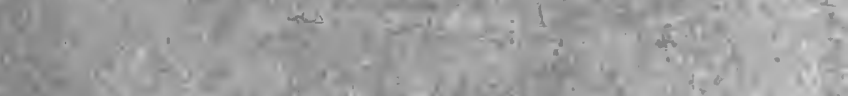

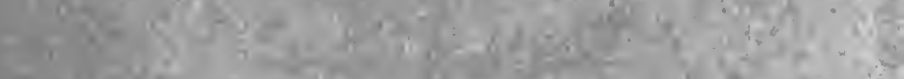

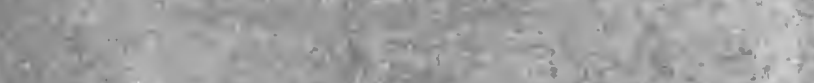

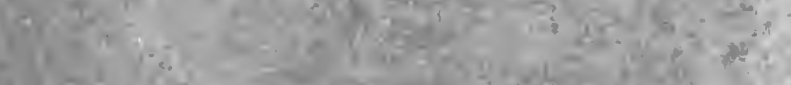

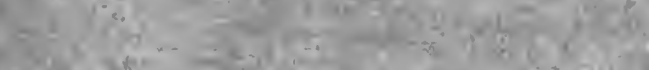
we.

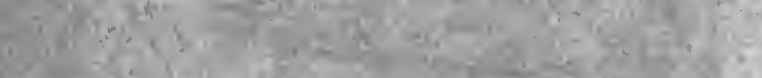

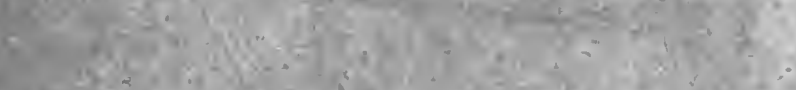

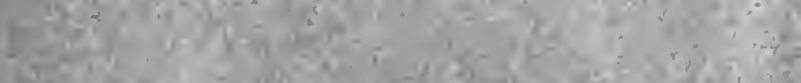

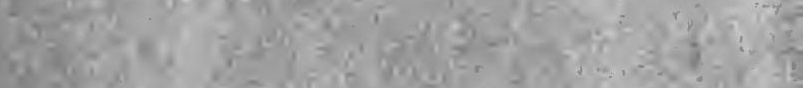

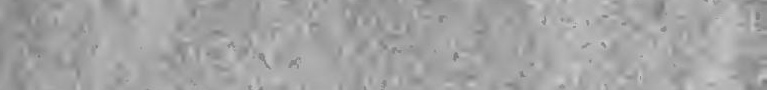

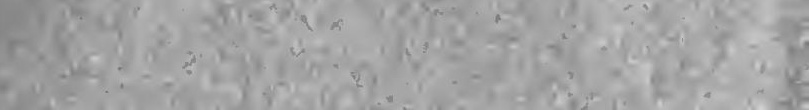

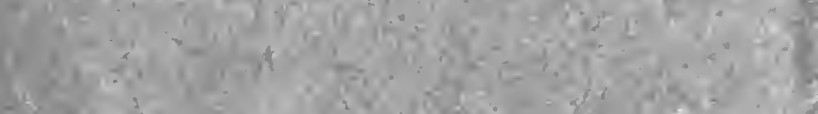

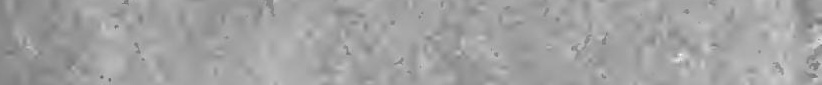

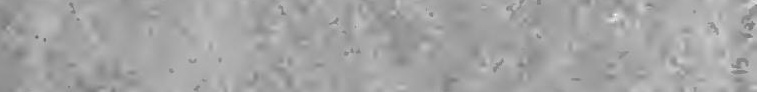

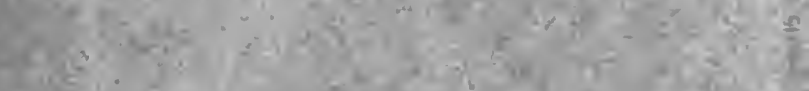

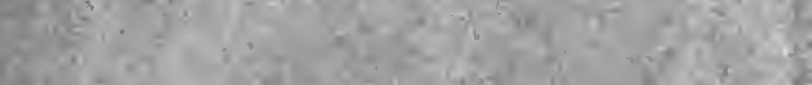

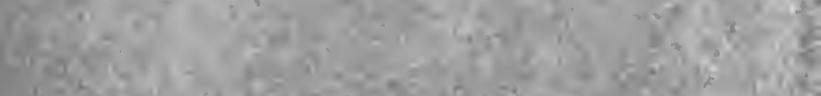

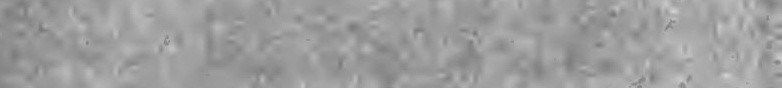

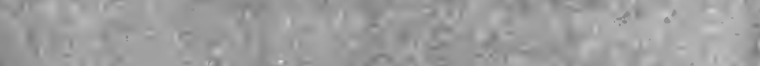
Q

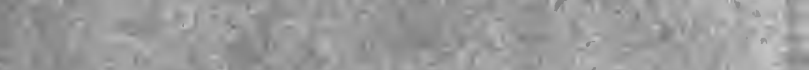

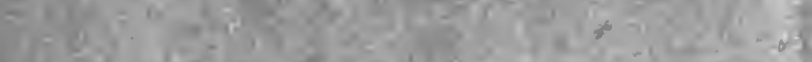

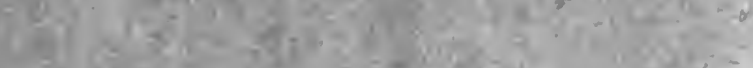




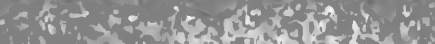

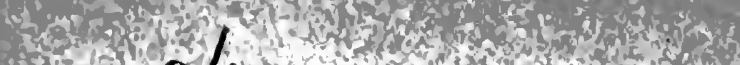

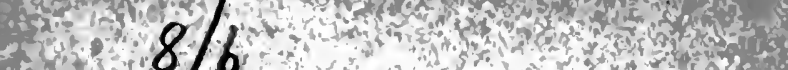

s.

\section{की \\ $\rightarrow \infty$}

tive

$-\frac{30}{x}$

$4 x^{2}, 3+3$

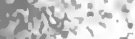

cotfo

Het?

in

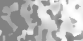

atset

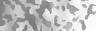

thy

$x^{2}{ }^{2} \geq 5$

tory

रet?

$5 \mathrm{St}$

बद

किश

itsos?

Then

anch

ajis:

atsin

48

$\therefore 115$

ris?

Sof?

$\operatorname{lig}^{2}$

af?

isti

us 3 -

"rint:

ing

axts

expor

and $25+2$

nets a

solver

+18.

$x^{2}+y^{2}-1=1$

at 2 th

$1030^{\circ}$

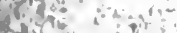

(a)

4)

efing

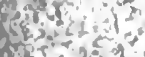

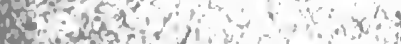
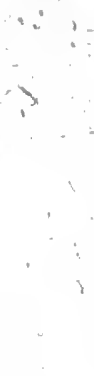

and

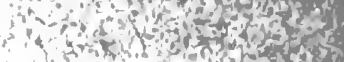

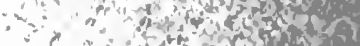

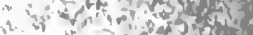

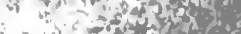

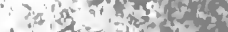

ant-intint

$i ;:-10 x=0$

$\rightarrow+1$ ches

$\dot{4}$

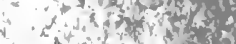

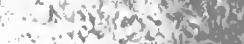

2 in

$\therefore$ hent?

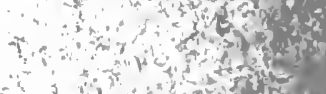

$\therefore$ a $x^{2} x^{2}$ तs

$\therefore x+x^{2}$ cthe

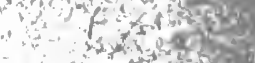

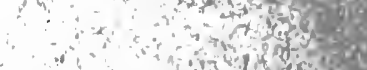

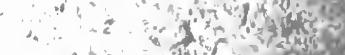

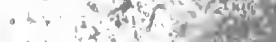

r.

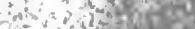

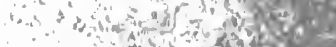

sin $x^{2}$

and $x^{2} \cdot 14$

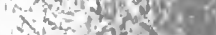

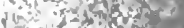

Ty $20 x$

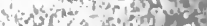

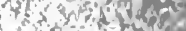

fon tist

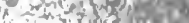

vi engtom

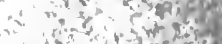

1 3 at

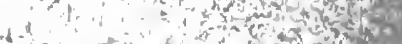

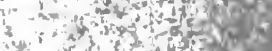

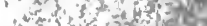

in

P:

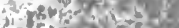

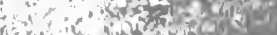

ro : c a

$\cdots+3,130$

ant as ast

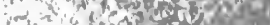

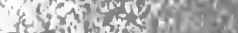

init bes 5

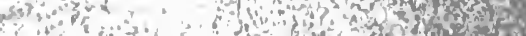




\section{APPLICATIONS OF DYNAMICS}

TO

PHYSICS AND CHEMISTRY. 


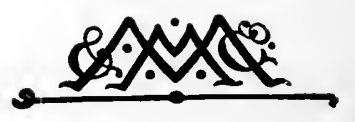


$T$

\section{APPLICATIONS OF DYNAMICS}

TO

\section{PHYSICS AND CHEMISTRY}

\section{J. J. THOMSON, M.A., F.R.S.}

FELLOW OF TRINITY COLLEGE AND CAVENDISH PROFESSOR OF EXPERIMENTAL PHYSICS, CAMBRIDGE.

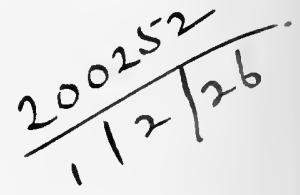

Zlonoron:

MACMILLAN AND CO.

AND NEW YORK.

\section{888}

[All Rights reserved.] 
Cambridge:

PRINTED BY C. J. CLAY, M.A. \& SONS, AT THE UNIVERSITY PRESS. 


\section{PREFACE.}

THE following pages contain the substance of a course of lectures delivered at the Cavendish Laboratory in the Michaelmas Term of 1886.

Some of the results have already been published in the Philosophical Transactions of the Royal Society for I 886 and I 887 , but as they relate to phenomena which belong to the borderland between two departments of Physics, and which are generally either entirely neglected or but briefly noticed in treatises upon either, I have thought that it might perhaps be of service to students of Physics to publish them in a more complete form. I have included in the book an account of some investigations published after the delivery of the lectures which illustrate the methods described therein.

There are two modes of establishing the connexion between two physical phenomena; the most obvious as well as the most interesting of these is to start with trustworthy theories of the phenomena in question and to trace every step of the connexion between them. This however is only possible in an exceedingly limited number of cases, and we are in general compelled to have recourse to the other mode in 
which by methods which do not require a detailed knowledge of the mechanism required to produce the phenomena, we show that whatever their explanation may be, they must be related to each other in such a way that the existence of the one involves that of the other.

It is the object of this book to develop methods of applying general dynamical principles for this purpose.

The methods I have adopted (of which that used in the first part of the book was suggested by Maxwell's paper on the Electromagnetic Field) make everything depend upon the properties of a single function of quantities fixing the state of the system, a result analogous to that enunciated by M. Massieu and Prof. Willard Gibbs for thermodynamic phenomena and applied by the latter in his celebrated paper on the "Equilibrium of Heterogeneous Substances" to the solution of a large number of problems in thermodynamics.

I wish in conclusion to thank my friend $\mathrm{Mr} \mathrm{L}$. R. Wilberforce, M.A., of Trinity College, for his kindness in correcting the proofs and for the many valuable suggestions he has made while the book was passing through the press.

$$
\text { J. J. THOMSON. }
$$

Trinity College, Cambridge, May 2nd, 1888. 


\section{CONTENTS.}

\section{CHAPTER I.}

Preliminary Considerations .

CHAPTER II.

The Dynamical Methods to be employed . $\quad$. $\quad$. $\quad$. 8

CHAPTER III.

Application of these Principles to Physics . . . . . 16

CHAPTER IV.

Discussion of the terms in the Lagrangian Function . . . $3^{\mathrm{I}}$

CHAPTER V.

Reciprocal Relations between Physical Forces when the Systems exerting them are in a Steady State . . . . 80

CHAPTER VI.

Effect of Temperature upon the Properties of Bodies • $\quad$ - 89

CHAPTER VII.

Electromotive Forces due to Differences of Temperature . . 106

CHAPTER VIII.

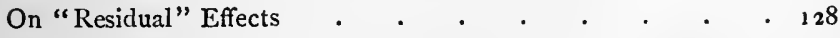


CHAPTER IX.

PAGE

Introductory to the Study of Reversible Scalar Phenomena . . 140

CHAPTER X.

Calculation of the mean Lagrangian Function

CHAPTER XI.

Evaporation

CHAPTER XII.

Properties of Dilute Solutions

CHAPTER XIII.

Dissociation

CHAPTER XIV.

General Case of Chemical Equilibrium

CHAPTER XV.

Effects produced by Alterations in the Physical Conditions on the

Coefficient of Chemical Combination . . . . $\quad 233$

CHAPTER XVI.

Change of State from Solid to Liquid . $\quad$. $\quad$. $\quad 243$

CHAPTER XVII.

The Connexion between Electromotive Force and Chemical Change $. \quad . \quad . \quad . \quad . \quad . \quad . \quad 26_{5}$

CHAPTER XVIII.

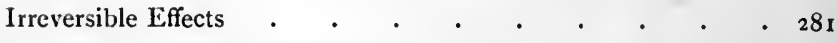




\title{
APPLICATIONS OF DYNAMICS TO PHYSICS AND CHEMISTRY.
}

\author{
CHAPTER I. \\ PRELIMINARY CONSIDERATIONS.
}

I. If we consider the principal advances made in the Physical Sciences during the last fifty years, such as the extension of the principle of the Conservation of Energy from Mechanics to Physics, the development of the Kinetic Theory of Gases, the discovery of the Induction of Electric Currents, we shall find that one of their most conspicuous effects has been to intensify the belief that all physical phenomena can be explained by dynamical principles and to stimulate the search for such explanations.

This belief which is the axiom on which all Modern Physics is founded has been held ever since men first began to reason and speculate about natural phenomena, but, with the remarkable exceptions of its successful application in the Corpuscular and Undulatory Theories of Light, it remained unfruitful until the researches of Davy, Rumford, Joule, Mayer and others showed that the kinetic energy possessed by bodies in visible motion can be very readily converted into heat. Joule moreover proved that whenever this is done the relation between the quantity of kinetic

T. D. 
energy which disappears and the quantity of heat which appears in consequence is invariable.

The ready conversion of kinetic energy into heat convinced these philosophers that heat itself is kinetic energy; and the invariable relation between the quantities of heat produced and of kinetic energy lost, showed that the principle of the Conservation of Energy, or of Vis-Viva as it was then called, holds in the transformation of heat into kinetic energy and vice versâ.

This discovery soon called attention to the fact that other kinds of energy besides heat and kinetic energy can be very readily converted from one form into another, and this irresistibly suggested the conclusion that the various kinds of energy with which we have to deal in Physics, such for example as heat and electric currents, are really fornis of kinetic energy - though the moving bodies which are the seat of this energy must be indefinitely small in comparison with the moving pieces of any machine with which we are acquainted.

These conceptions were developed by several mathematicians but especially by v. Helmholtz, who, in his treatise Ueber die Erhaltung der Kraft, Berlin, 1847, applied the dynamical method of the Conservation of Energy to the various branches of physics and showed that by this principle many well-known phenomena are connected with each other in such a way that the existence of the one involves that of the other.

2. The case which from its practical importance at first attracted the most attention was that of the transformation of heat into other forms of energy and vice versâ.

In this case it was soon seen that the principle of the Conservation of Energy-the First Law of Thermodynamics as it was called-was not sufficient to obtain all the relations 
existing between the effects of heat on the various properties of a body and the heat produced or absorbed when certain changes take place in the body, but that these relations could be deduced by the help of another principle, the Second Law of Thermodynamics-which states that if to a system where all the actions are perfectly reversible a quantity of heat $d Q$ be communicated at the absolute temperature $\theta$, then

$$
\int \frac{d Q}{\theta}=0
$$

the integration being extended over any complete cycle of operations.

This statement is founded on various axioms by different physicists, thus for example Clausius bases it upon the "axiom" that heat cannot of itself pass from one body to another at a higher temperature, and Sir William Thomson on the "axiom" that it is impossible by means of inanimate material agency to derive mechanical effect from any portion of matter by cooling it below the temperature of the coldest of the surrounding objects.

Thus the Second Law of Thermodynamics is derived from experience and is not a purely dynamical principle.

We might have expected $a$ priori from dynamical considerations that the principle of the Conservation of Energy would not be sufficient by itself to enable us to deduce all the relations which exist between the various properties of bodies. For this principle is rather a dynamical result than a dynamical method and in general is not sufficient by itself to solve completely any dynamical problem.

Thus we could not expect that for the dynamical treatment of Physics the principle of the Conservation of Energy would be sufficient by itself, since it is not so in the much simpler cases which occur in ordinary Mechanics. 
The resources of dynamics however are not exhausted even though the principle of the Conservation of Energy has been tried. Fortunately we possess other methods, such as Hamilton's principle of Varying Action and the method of Lagrange's Equations, which hardly require a more detailed knowledge of the structure of the system to which they are applied than the Conservation of Energy itself and yet are capable of completely determining the motion of the system.

3. The object of the following pages is to endeavour to see what results can be deduced by the aid of these purely dynamical principles without using the Second Law of Thermodynamics.

The advantages of this method in comparison with that of the two laws of Thermodynamics are

(I) that it is a dynamical method, and so of a much more fundamental character than that involving the use of the Second Law ;

(2) that one principle is sufficient instead of two;

(3) that the method can be applied to questions in which there are no transformations of other forms of energy from or into heat (except the unavoidable ones due to friction), while for this case the other method degenerates into the principle of the Conservation of Energy, which is often not sufficient to solve the problem.

The disadvantages of the method on the other hand are that, since the method is a dynamical one, the results are expressed in terms of dynamical quantities, such as energy, momentum, or velocity, and so require further knowledge before we can translate them in terms of the physical quantities we wish to measure, such as intensity of a current, temperature, and so on: a knowledge which in all cases we do not possess. 
The Second Law of Thermodynamics, on the other hand, being based on experience does not involve any quantity which cannot be measured in the Physical Laboratory.

For this reason there are some cases where the Second Law of Thermodynamics leads to more definite results than the dynamical methods of Hamilton or Lagrange. Even here I venture to think the results of the application of the dynamical method will be found interesting, as they show what part of these problems can be solved by dynamics, and what has to be done by considerations which are the results of experience.

4. Many attempts have been made to show that the Second Law of Thermodynamics is a consequence of the principle of Least Action; none of these proofs seem quite satisfactory; but even if the connexion had been proved in an unexceptionable way it would still seem desirable to investigate the results of applying the principle of Least Action, or the equivalent one of Lagrange's Equations, directly to various physical problems.

If these results agree with those obtained by the use of the Second Law of Thermodynamics, it will be a kind of practical proof of the connexion between this law and the principle of Least Action.

5. Considering our almost complete ignorance of the structure of the bodies which form most of the dynamical systems with which we have to deal in physics, it might seem a somewhat unpromising undertaking to attempt to apply dynamics to such systems. But we must remember that the object of this application is not to discover the properties of such systems in an altogether $a$ priori fashion, but rather to predict their behaviour under certain circumstances after having observed it under others.

A dynamical example may illustrate what the application 
of dynamics to physical problems may be expected to do, and the way in which it is likely to do it. Let us suppose that we have a number of pointers on a dial, and that behind the dial the various pointers are connected by a quantity of mechanism of the nature of which we are entirely ignorant. Then if we move one of the pointers, $A$ say, it may happen that we set another one, $B$, in motion.

If now we observe how the velocity and position of $B$ depend on the velocity and position of $A$, we can by the aid of dynamics foretell the motion of $A$ when the velocity and position of $B$ are assigned, and we can do this even though we are ignorant of the nature of the mechanism connecting the two pointers. Or again we may find that the motion of $B$ when $A$ is assigned depends to some extent upon the velocity and position of a third pointer $C$ : if in this case we observe the effect of the motion of $C$ upon that of $A$ and $B$ we may deduce by dynamics the way in which the motion of $C$ will be affected by the velocities and positions of the pointers $A$ and $B$.

This illustrates the way in which dynamical considerations may enable us to connect phenomena in different branches of physics. For the observation of the motion of $B$ when that of $A$ is assigned may be taken to represent the experimental investigation of some phenomenon in Physics, while the deduction by dynamics of the motion of $A$ when that of $B$ is assigned may represent the prediction by the use of Hamilton's or Lagrange's principle of a new phenomenon which is a consequence of the one investigated experimentally.

Thus to take an illustration, suppose we investigate experimentally the effect of a current of electricity both steady and variable upon the torsion of a longitudinally magnetized iron wire along which the current flows, then we 
can deduce by dynamics the effects of torsion and variations of torsion in the wire upon a current flowing along it.

The method is really equivalent to an extension and generalization of the principle of the equality of action and reaction, as when we have two bodies $A$ and $B$ acting upon each other if we observe the motion of $B$ which results when $A$ moves in a known way we can deduce by the aid of this principle the motion of $A$ when that of $B$ is known. The more general case which we have to consider in Physics is when instead of two bodies attracting each other we have two phenomena which mutually influence each other. 


\section{CHAPTER II.}

THE DYNAMICAL METHODS TO BE EMPLOYED.

6. As we do not know the nature of the mechanism of the physical systems whose action we wish to investigate, all that we can expect to get by the application of dynamical principles will be relations between various properties of bodies. And to get these we can only use dynamical methods which do not require an intimate knowledge of the system to which they are applied.

The methods introduced by Hamilton and Lagrange possess this advantage and, as they each make the behaviour of the system depend upon the properties of a single function, they reduce the subject to the determination of this function. In general the way that we are able to connect various physical phenomena is by seeing from the behaviour of the system under certain circumstances that there must be a term of a definite kind in this function, the existence of this term will then often by the application of Lagrangian or Hamiltonian methods point to other phenomena besides the one that led to its detection.

7. We shall now for convenience of reference collect the dynamical equations which we shall most frequently have to use.

The most generally useful method is Hamilton's principle 
of Varying Action according to which (see Routh's Advanced Rigid Dynamics, p. 245)

$$
\delta \int_{t_{0}}^{t_{1}}(T-V) d t=\left\{\mathbf{\Sigma} \frac{d T}{d \dot{q}} \delta q\right\}_{t_{0}}^{t_{1}} \ldots \ldots . .(\mathrm{r}),
$$

where $T$ and $V$ are respectively the kinetic and potential energies of the system, $t$ the time, and $q$ a coordinate of any type. In this case $t_{0}$ and $t_{1}$ are each supposed to be constant.

In some cases it is convenient to use the equation in this form but in others it is more convenient to use Lagrange's Equations, which may be derived from equation (I) (Routh's Advanced Rigid Dynamics, p. 249) and which may be written in the form

$$
\frac{d}{d t} \frac{d L}{d \dot{q}}-\frac{d L}{d q}=Q \ldots \ldots \ldots \ldots \ldots \ldots(2) .
$$

where $L$ is written for $T-V$ and is called the Lagrangian function and $Q$ is the external force acting on the system tending to increase $q$.

In the preceding equations the kinetic energy is supposed to be expressed in terms of the velocities of the coordinates. In many cases however instead of working with the velocities corresponding to all the coordinates it is more convenient to work with the velocities corresponding to some coordinates but with the momenta corresponding to the others. This is especially convenient when some of the coordinates only enter the Lagrangian function through their differential coefficients and do not themselves occur explicitly in this function. In a paper "On some Applications of Dynamical Principles to Physical Phenomena" (Phil. Trans. 1885, Part 11.) I have called these "kinosthenic" coordinates. In the following pages the term "speed coordinates" will for the sake of brevity be used instead wherever it will not lead to ambiguity. 
The most important property of such a coordinate is that whenever no external force of its type acts upon the system, the momentum corresponding to it is constant.

For if $\chi$ be a speed coordinate, since

$$
\frac{d L}{d x}=0,
$$

we have by Lagrange's equation since no external force acts on the system

$$
\frac{d}{d t} \frac{d L}{d \dot{\chi}}=0
$$

as the momentum corresponding to $\chi$ is $d L / d \dot{\chi}$, this equation shows that it is constant.

8. Routh (Stability of Motion, p. 6r) has given a general method which enables us to use the velocities of some coordinates and the momenta corresponding to the remainder, and which is applicable whether these latter coordinates are speed coordinates or not.

The method is as follows: suppose that we wish to use the velocities of the coordinates $q_{1}, q_{2} \ldots$ and the momenta corresponding to the coordinates $\phi_{1}, \phi_{2} \ldots$ then Routh has shown that if we use instead of $L$ the new function $L^{\prime}$ given by the equation.

$$
L^{\prime}=L-\dot{\phi}_{1} \frac{d T}{d \dot{\phi}_{1}}-\dot{\phi}_{2} \frac{d T}{d \dot{\phi}_{2}}-\& \mathrm{c}
$$

and eliminate $\dot{\phi}_{1}, \dot{\phi}_{2} \ldots$ by means of the equations

$$
\Phi_{1}=\frac{d T}{d \dot{\phi}_{2}}, \Phi_{2}=\frac{d T}{d \dot{\phi}_{2}}, \ldots \& c .
$$

then as far as the coordinates $q_{1}, q_{2}$, are concerned we may use Lagrange's equations if we substitute $L^{\prime}$ for $L$. Thus we have a series of equations of the type

$$
\frac{d}{d t} \frac{d L^{\prime}}{d \dot{q}}-\frac{d L^{\prime}}{d q}=Q
$$


If we call

$$
\frac{1}{2} \dot{\phi}_{1} \frac{d T}{d \dot{\phi}_{1}}
$$

the part of the kinetic energy corresponding to the coordinate $\phi_{1}$ then we see by (4) that $L^{\prime}=$ the kinetic energy of the system minus its potential energy minus twice the kinetic energy corresponding to the coordinates whose velocities are eliminated.

9. If we do not know the structure of this system all that we can determine by observing its behaviour will be the Lagrangian function or its modified form, and since this function completely determines the motion of the system it is all we require for the investigation of its properties. We see however that when we calculate the "energy" corresponding to any physical condition the interpretation may be ambiguous if the energy is not entirely potential. For what we really calculate is the Lagrangian function or its modified form and this is the kinetic energy minus the potential energy minus twice the kinetic energy corresponding to the coordinates whose velocities are eliminated. So that the term in the energy which we have calculated may be any one of these three things. Thus to take an example, it is said that the energy of a piece of soft iron of unit volume, throughout which the intensity of magnetization is uniform and equal to $I$, is $-I^{2} / 2 k$, where $k$ is the coefficient of magnetic induction of the iron, but all that this means is that the term $I^{2} / 2 k$ occurs in the Lagrangian function (modified or otherwise) of the system whose motion or configuration produces the phenomenon of magnetization. And without further considerations we do not know whether this represents an amount of kinetic energy $I^{2} / 2 k$ or potential energy $-I^{9} / 2 k$, or some kinetic energy corresponding to coordinates whose 
velocities have been eliminated, or some combination of all three of these.

I0. This ambiguity however does not occur if we have the system completely mapped out by coordinates, because in this case whenever we find a term in the Lagrangian function it must be expressed in terms of these coordinates and their velocities, or it may be the momenta corresponding to them, and we can tell by inspection whether the term expresses kinetic or potential energy. Two investigations in the second volume of Maxwell's Electricity and Magnetism afford a good illustration of the way in which this ambiguity is cleared away by an increase in the precision of our ideas about the configuration of the system. In the early part of the volume by considering the mechanical forces between two circuits carrying electric currents, it is shown that two such circuits conveying currents $i, j$ possess a quantity of potential energy $-M i j$ where $M$ is a quantity depending on the shape and size of the two circuits and their relative position. Later on however when coordinates capable of fixing the electrical configuration of the system have been introduced it is shown that the system instead of possessing $-M i j$ units of potential energy really possesses $+M i j$ units of kinetic.

I I. The following considerations may be useful as helping to show that this ambiguity is largely verbal and is probably mainly due to our ignorance of what potential energy really is.

Suppose that we have a system fixed by $n$ coordinates, $q_{1}, q_{2}, \ldots q_{n}$ of the ordinary kind, that is, coordinates which occur explicitly in the expressions for the kinetic or potential energies, and which we shall call positional coordinates, and $m$ kinosthenic or speed coordinates $\phi_{1}, \phi_{2}, \ldots \phi_{m}$. Let us further suppose that there are no terms in the expression for the kinetic energy which involve the product of the 
velocity of a $q$ and a $\phi$ coordinate and that the system has no potential energy.

Then by Routh's method we can use Lagrange's equation for the $q$ coordinates if instead of the ordinary Lagrangian function $L$ which reduces in this case to the kinetic energy we use the modified function $L^{\prime}$ given by the equation

$$
L^{\prime}=L-\Sigma \dot{\phi} \frac{d T}{d \dot{\phi}} \ldots \ldots \ldots \ldots \ldots \ldots \ldots(6),
$$

or,

$$
L^{\prime}=\frac{1}{2} \Sigma \dot{q} \frac{d T}{d \dot{q}}-\frac{1}{2} \Sigma \dot{\phi} \frac{d T}{d \dot{\phi}}
$$

and where $\dot{\phi}_{1}, \dot{\phi}_{2}$ are to be eliminated by the aid of the equations

$$
\Phi_{1}=\frac{d T}{d \phi_{1}}
$$

Thus since the expression for $L$ does not contain any terms involving the product of the velocity of a $q$ and a $\phi$ coordinate, $L^{\prime}$ will be of the form

$$
T_{(q q)}-T_{(\phi \phi)}
$$

where $T_{(\varphi q)}$ is the kinetic energy arising from the motion of the $q$ or positiunal coordinates, $T_{(\phi \phi)}$ that arising from the motion of the kinosthenic or speed coordinates.

By Routh's modification of the. Lagrangian equations we have

$$
\frac{d}{d !} \frac{d}{d \dot{q}_{1}}\left(T_{(q q)}-T_{(\phi \phi)}\right)-\frac{d}{d q_{1}}\left(T_{(q q)}-T_{(\phi \phi)}\right)=0 \ldots(8),
$$

but

$$
\frac{d T_{(\phi \phi)}}{d \dot{q}_{1}}=0
$$

so that equation (8) reduces to

$$
\frac{d}{d t} \frac{d T_{(q q)}}{d \dot{q}_{1}}-\frac{d T_{(q q)}}{d q_{1}}=-\frac{d T_{(\phi \phi)}}{d q_{1}}
$$


If the system fixed by the positional coordinates $q$ had possessed a quantity of potential energy equal to $V$, the equations of motion would have been of the type,

$$
\frac{d}{d t} \frac{d T_{(q q)}}{d \dot{q}_{1}}-\frac{d T_{(q q)}}{d q_{1}}=-\frac{d V}{d q_{1}} \ldots \ldots \ldots \ldots(\mathrm{1}) .
$$

By comparing equations (9) and (10) we see that the system fixed by the positional coordinates $q$ will behave exactly like a system whose kinetic energy is $T_{(q q)}$ and whose potential energy is $T_{(\phi \phi)}$.

Thus we may look on the potential energy of any system as kinetic energy arising from the motion of systems connected with the original system-the configurations of these systems being capable of being fixed by kinosthenic or speed coordinates.

Thus from this point of view all energy is kinetic, and all terms in the Lagrangian function express kinetic energy, the only thing doubtful being whether the kinetic energy is due to the motion of ignored or positional coordinates; this can however be determined at once by inspection.

12. Some of the theorems in dynamics become very much simpler from this point of view. Let us take for example the principle of Least Action-that for the unconstrained motion of a system whose energy remains constant

$$
\int_{t_{0}}^{t_{1}} T d t
$$

is a minimum from one configuration to another-and apply it to the system we have been considering in which all the energy is kinetic but some of it is due to the motion of a system whose configuration can be fixed by kinosthenic coordinates.

As all the energy is kinetic its magnitude remains constant by the principle of the Conservation of Energy, 
and so the principle of Least Action takes the very simple form, that with a given quantity of energy any material system will by its unguided motion go along the path which will take it from one configuration to another in the least possible time. The material system must of course include the kinosthenic systems whose motion produces the same effect as the potential energy of the original system; and two configurations are not supposed to coincide unless the configuration of these kinosthenic systems coincide also.

This view which regards all potential energy as really kinetic has the advantage of keeping before us the idea that it is one of the objects of Physical Science to explain natural phenomena by means of the properties of matter in motion. When we have done this we have got a complete physical explanation of any phenomenon and any further explanation must be rather metaphysical than physical. It is not so however when we explain the phenomenon as due to changes in the potential energy of the system; for potential energy cannot be said, in the strict sense of the term, to explain anything. It does little more than embody the results of experiments in a form suitable for mathematical investigations.

The matter whose motion constitutes the kinetic energy of the kinosthenic systems, the " $\phi$ " systems, which we regard as the potential energy of the " $q$ " systems, may be either that of parts of the system, or the surrounding ether, or both; in many cases we should expect it to be mainly the ether. 


\section{CHAPTER III.}

APPLICATION OF THESE PRINCIPLES TO PHYSICS.

13. IN our applications of Dynamics to Physics it will be well to begin with the cases which are the most nearly allied to those we consider in ordinary Rigid Dynamics. Now in this subject when there is no friction all the motions are reversible and are chiefly relations between vector quantities. We shall therefore begin by considering reversible vector effects and afterwards go on to reversible effects involving scalar as well as vector relations; those for example in which a scalar quantity such as temperature is prominently involved: lastly we shall consider irreversible effects. Thus the order in which we shall consider the subject will be

r. Reversible vector phenomena.

2. Reversible scalar phenomena.

3. Irreversible phenomena.

14. We shall begin by considering the relations between the phenomena in elasticity, electricity, and magnetism and the way in which these depend upon the motion and configuration of the bodies which exhibit the phenomena.

These phenomena differ from some we shall consider later on in that we have the quantities concerned in them entirely under our control and can by applying proper 
external forces make them take any value we please (subject of course to such limitation as the strength of the material and the saturation of magnets may impose). The other phenomena on the other hand depend upon a multitude of coordinates over whose individual motion we have no control though we have some over their average motion. As the first kind of phenomena most closely resemble those we have to do with in ordinary dynamics we shall begin with them.

15. The first thing we have to do when we wish to apply dynamical methods to investigate the motion of a system is to choose coordinates which can fix its configuration.

We shall find it necessary to give a more general meaning to the term "coordinate" than that which obtains in ordinary Rigid Dynamics. There a coordinate is a geometrical quantity helping to fix the geometrical configuration of the system.

In the applications of Dynamics to Physics however, the configurations of the systems we consider have to be fixed, with respect to such things as distributions of electricity and magnetism, for example, as well as geometrically, and to do this we have in the present state of our knowledge to use quantities which are not geometrical.

Again the coordinates which fix the configurations of the systems in ordinary dynamics are sufficient to fix them completely, while we may feel pretty sure that the coordinates which we use to fix the configuration of the system with respect to many of its physical properties, though they may fix it as far as we can observe it, are not sufficient to fix it in every detail; that is they would not be sufficient to fix it if we had the power of observing differences whose fineness was comparable with that of molecular structure.

T. D. 
Hydrodynamics furnishes us with many very good illustrations of this latter point. For example, when a sphere moves through an incompressible fluid, we can express the kinetic energy of the system comprising both sphere and fluid in terms of the differential coefficients of the three coordinates which fix the centre of the sphere, though it would require a practically infinite number of coordinates to fix the configuration of the fluid completely.

Now Thomson and Tait (Natural Philosophy, vol. 1. p. 320) have shown how we can "ignore" these coordinates when the kinetic energy can be expressed without them, and that we may treat the system as if it were fully determined by the coordinates in terms of whose differential coefficients the kinetic energy is expressed.

And again Larmor (Proceedings of London Mathematical Society, xv. p. 173) has proved that if $L^{\prime}$ be Routh's modification of the Lagrangian function, that is $q_{1}, q_{2}, \ldots$ being the coordinates retained, $\phi_{1}, \phi_{2}, \ldots$ those ignored, $Q_{1}$, $Q_{2} \ldots, \Phi_{1}, \Phi_{2}$, the momenta corresponding to these coordinates respectively, if

then

$$
\begin{aligned}
& L^{\prime}=\frac{1}{2}\{\Sigma Q \dot{q}-\Sigma \Phi \dot{\phi}\}-V, \\
& \quad \delta \int_{t_{0}}^{t_{1}} L^{\prime} d t=0 \ldots \ldots \ldots \ldots \ldots(\mathrm{I} \mathrm{r}) .
\end{aligned}
$$

If all the kinetic energy vanishes when the positional coordinates $q_{1}, q_{2}, \ldots$ are constant, as is the case when a number of solids move through a perfect fluid in which there is no circulation, $L^{\prime}$ is the difference between the kinetic and potential energies of the system. If however the kinetic energy does not vanish when the velocities of the positional coordinates all vanish, as for example when a number of solids are moving through a fluid in which there is circulation, $L^{\prime}$ no longer equals the difference between the kinetic and potential energies of the system. 
It follows from ( $\mathrm{I} x$ ), by the Calculus of Variations, that if $L^{\prime}$ be expressed in terms of a series of quantities $q_{1}, q_{2}, \ldots$ and their first differential coefficients, then whatever these quantities may be, we must have a series of equations of the type

$$
\frac{d}{d t} \frac{d L^{\prime}}{d \dot{q}}-\frac{d L^{\prime}}{d q}=0 \ldots \ldots \ldots \ldots \ldots . . .(\mathrm{r} 2) .
$$

Thus we see that we may treat any variable quantities as coordinates if the modified Lagrangian function can be expressed in terms of them and their first differential coefficients. We shall take this as our definition of a coordinate.

r6. When we introduce a symbol to fix a physical quantity we may not at first sight be sure whether it is a coordinate or the differential coefficient of one with respect to the time.

For example, we might feel uncertain whether the symbol representing the intensity of a current was a coordinate or the differential coefficient of one. The simplest dynamical considerations however will enable us to overcome this difficulty. Thus if when there is no dissipation of energy by irreversible processes, the quantity represented by the symbol remains constant under the action of a constant force tending to alter its value, the energy at the same time remaining constant, then the symbol is a coordinate.

Again, if it remains constant and not zero when no force acts upon it, there being no dissipation and the energy remaining constant, the symbol represents a velocity, that is, the differential coefficient of a coordinate with respect to the time.

Let us apply these considerations to the example mentioned above; as the intensity of a current flowing through a perfect conductor, the only circumstances under which there is no dissipation, does not satisfy the first of these 
conditions, while it does satisfy the second, we conclude that the intensity of a current ought to be represented by the rate of change of a coordinate and not by the coordinate itself.

\section{Specification of Coordinates.}

17. To fix the configuration of the system so far as the phenomena we are considering are concerned we shall use the following kinds of coordinates.

(I) Coordinates to fix the geometrical configuration of the system, i.e. to fix the position in space of any bodies of finite size which may be in the system. For this purpose we shall use the coordinates ordinarily used in Rigid Dynamics and denote them by the letters $x_{1}, x_{2}, x_{3} \ldots$; and when we want to denote a geometrical coordinate generally without reference to any one in particular we shall use the letter $x$.

(2) Coordinates to fix the configuration of the strains in the system. We shall use for this purpose, as is ordinarily done in treatises on elasticity, the components parallel to the axes of $x, y, z$ of the displacements of any small portion of the body, and denote them by the letters $\alpha, \beta, \gamma$ respectively. For the strains

$$
\begin{gathered}
\frac{d a}{d x}, \frac{d \beta}{d y}, \frac{d \gamma}{d z} \\
\left(\frac{d \gamma}{d y}+\frac{d \beta}{d z}\right),\left(\frac{d a}{d z}+\frac{d \gamma}{d x}\right),\left(\frac{d \beta}{d x}+\frac{d a}{d y}\right),
\end{gathered}
$$

we shall use the letters $e, f, g, a, b, c$ respectively. It will be convenient to have a letter typifying these quantities generally without reference to any one in particular, we shall use the letter $w$ for this purpose.

(3) Coordinates to fix the electrical configuration of the system. For this purpose we shall use coordinates 
denoted by the letiers $y_{1}, y_{2} \ldots$, the typical coordinate being denoted by $y$, where $y$ in a dielectric is what Maxwell calls an electric displacement, and in a conductor the time integral of a current flowing through some definite area.

(4) Coordinates to fix the magnetic configuration. We might do this by specifying the intensity of magnetization at each point, but it is clearer I think to regard the magnetic configuration as depending, even in the simplest case, upon two coordinates, one of which is a kinosthenic or speed coordinate.

This way of looking at it brings it into harmony with the two most usual ways of representing the magnetization of a body, viz. Ampère's theory and the hypothesis of Molecular Magnets.

According to Ampère's theory the magnetization is due to electric currents flowing through perfectly conducting circuits in the molecules of the magnets. In this case the differential coefficient of the kinosthenic coordinate would fix the intensity of the current, and the other coordinate the orientation of the planes of the circuits.

According to the Molecular Magnet theory, any magnet of finite size is built up of a large number of small magnets arranged in a polarized way. Here the momentum corresponding to the kinosthenic or speed coordinate may be regarded as fixing the magnetic moment of a little magnet, which it is well fitted to do by its constancy; the other coordinate may be regarded as fixing the arrangement of the little magnets in space.

We shall denote the kinosthenic coordinate by $\zeta$ and the geometrical one by $\eta$ and suppose that they are so chosen that the intensity of magnetization at any point is $\eta \xi$, where $\xi$ is the momentum corresponding to the kinosthenic coordinate $\zeta . \quad \eta$ is a vector quantity and may be resolved 
into three components parallel to the axes of $x, y, z$ respectively.

18. Having chosen the coordinates there are two ways in which we may proceed. We may either write down the most general expression for the Lagrangian function in terms of these coordinates and their differential coefficients, and then investigate the physical consequences of each term in this expression. If these consequences are contradicted by experience we conclude that the term we are considering does not exist in the expression for the Lagrangian function.

Or we may know as the result of experiment that there must be a certain term in the expression for the Lagrangian function and proceed by the application of Lagrange's Equations to develop the consequences of its existence. Thus, for example, we know by considering the amount of work required to establish the electric field that there must be in the Lagrangian function of unit volume of the dielectric a term of the form

$$
-\frac{\mathbf{r}}{8 \pi K} D^{2}
$$

where $K$ is the specific inductive capacity of the dielectric and $D$ the resultant electric displacement. We can then by applying Lagrange's equation to this term see what are the consequences of the specific inductive capacity of the dielectric being altered by strain (see $\S 39$ ).

We shall make use of both methods but commence with the first as being perhaps the most instructive, and also because we shall have a great many examples of the second method later on since the scalar phenomena do not admit of being treated by the first method.

19. In using the first method the first thing we have to do is to write down the most general expression for the Lagrangian function in terms of the coordinates $x, y, \eta, \zeta, w$. 
Let us suppose that $\dot{\zeta}$ is eliminated by means of the equation

$$
\frac{d T}{d \dot{\zeta}}=\xi
$$

and that we work with Routh's modification of the Lagrangian function.

The most general expression for the terms corresponding to the kinetic part of this function, which is the only part we can typify, is of the form

$$
\begin{aligned}
& \frac{1}{2}\left\{\left(x_{1} x_{1}\right) \dot{x}_{1}^{2}+\left(x_{2} x_{2}\right) \dot{x}_{2}^{2}+2\left(x_{1} x_{2}\right) \dot{x}_{1} \dot{x}_{2}+\ldots \ldots\right. \\
& \quad+\left(y_{1} y_{1}\right) \dot{y}_{1}^{2}+\left(y_{2} y_{2}\right) \dot{y}_{2}^{2}+2\left(y_{1} y_{2}\right) \dot{y}_{1} \dot{y}_{2}+\ldots \\
& \quad+(w w) \dot{w}^{2}+(\eta \eta) \dot{\eta}^{2}+(\xi \xi) \xi^{2} \\
& \quad+2(x y) \dot{x} \dot{y}+2(x w) \dot{x} \dot{w}+2(x \eta) \dot{x} \dot{\eta} \\
& \quad+2(x \xi) \dot{x} \xi+2(y w) \dot{y} \dot{w}+2(y \eta) \dot{y} \dot{\eta} \\
& \quad+2(y \xi) \dot{y} \xi+2(w \eta) \dot{w} \dot{\eta}+2(w \xi) \dot{w} \xi \\
& \quad+2(\eta \xi) \dot{\eta} \xi\} .
\end{aligned}
$$

These terms may be divided into fifteen types.

There are five sets which are quadratic functions of the velocity or momentum corresponding to one kind of coordinate. Each of these five sets must exist in actual physical systems if there is anything analogous to inertia in the phenomena which the corresponding coordinates typify.

Again, there are ten sets of terms of the type

$$
(x y) \dot{x} \dot{y} \text { or }(x \xi) \dot{x} \xi \text {, }
$$

involving the product of two velocities or a velocity and a momentum of two coordinates of different kinds.

To determine whether any particular term of this type exists or not we must determine what the physical consequences of it would be; if these are found to be contrary to experience we conclude that this term does not exist. 
20. We can determine the consequences of the existence of a term of this kind in the expression for the kinetic energy in the following way.

Let us suppose that we have a term in the modified Lagrangian function of the type

$$
\text { ( } p q) \ddot{p q} \text {, }
$$

where $p$ and $q$ may be any of the five kinds of coordinates we are considering.

Then we have by Routh's modification of Lagrange's equations

$$
\frac{d}{d t} d \dot{p}-\frac{d L^{\prime}}{d p}=P
$$

where $P$ is the external force of type $p$ acting on the system.

Thus the effect of the term

\section{$(p q) \dot{p} \dot{q}$}

is equivalent to the existence of a force of the type $p$ equal to

that is,

$$
\frac{d}{d p}\{(p q) \ddot{p q}\}-\frac{d}{d t}\{(p q) \dot{q}\},
$$

$$
-\left\{(p q) \ddot{q}+\frac{d}{d q}(p q) \dot{q}^{2}+\Sigma \frac{d}{d r}(p q) \dot{r} \dot{q}\right\} \ldots \ldots(\mathbf{1} 4) ;
$$

a force of type $q$ equal to

that is,

$$
\frac{d}{d q}\{(p q) \ddot{p} q\}-\frac{d}{d t}\{(p q) \dot{p}\},
$$

$$
-\left\{(p q) \ddot{p}+\frac{d}{d p}(p q) \dot{p}^{2}+\Sigma \frac{d}{d r}(p q) \ddot{r p}\right\} \ldots . . .\left(\text { I }_{5}\right) ;
$$

and a force of type $r$ equal to

$$
\frac{d}{d r}\{(p q) \ddot{p} q\}
$$


that is,

$$
\ddot{p q} \frac{d}{d r}(p q)
$$

where $r$ is a coordinate of any type other than that of $p$ or $q$.

Each of the terms in these expressions would correspond to some physical phenomenon; and as it is clearer to take a definite case to illustrate this, let us suppose that $p$ is the geometrical coordinate symbolized by $x$, and $q$ the electrical coordinate $y$.

Then if the term $(x y) \dot{x} \dot{y}$ occurred in the expression for the Lagrangian function, the mechanical force produced by a steady current would not be the same as that produced by a variable one momentarily of the same intensity. This is so because by the expression (I 14 ) there is the term

\section{$(x y) \ddot{y}$}

in the expression for the force of type $x$, that is the mechanical force, and as $\ddot{y}$ is zero if the current is steady, there would be a mechanical force depending on the rate of variation of the current if this term existed.

Again, we see from the term $\frac{d}{d y}(x y) \dot{y}^{2}$ in the expression (14), remembering that $p$ stands for $x$ and $q$ for $y$, that if $(x y)$ were a function of $y$ the current would produce a mechanical force proportional to its square, so that the force would not be reversed if the direction of the current was reversed.

Or again, if we consider the expression for the force of type $y$ or $q$, that is the electromotive force, we see that the existence of this term implies the production of an electromotive force by a body whose velocity is changing, depending upon the acceleration of the body; this is shown by the existence of the term (xy) $\ddot{x}$ in (I 5 ), the expression for the electromotive force. 
If $(x y)$ were a function of $x$, the term $(x y) \dot{x}^{2}$ in (15) shows that a moving body would produce an electromotive force proportional to the square of its velocity, and therefore one that would not be reversed when the direction of motion of the body was reversed.

As none of these effects have been observed we conclude that this term does not exist in the expression for the Lagrangian function of a physical system (see Maxwell, Electricity and Magnetism, $\$ 574$ ).

21. We shall now go through the various types of terms which involve the product of the velocities of two coordinates of different kinds, or a velocity of one kind and the momentum of another, in order to see whether they exist or not in the expression for the Lagrangian function.

The reasoning to be used is of the same nature as that just given, and we may leave it to the reader to show by the consideration of the expressions (I4) and (I5) that the existence of the several terms carries with it the consequences we describe.

Taking the terms in order we have

I. Terms of the form

$$
\text { (xy) } \dot{x} \dot{y} \text {. }
$$

We have just seen that terms of this kind cannot exist in the expression for the Lagrangian function. See also Maxwell, Electricity and Magnetism, II. part Iv. chap. 7 .

2. Terms of the form

$$
(x w) \dot{x} \dot{i} \cdot
$$

Terms of this form may exist in the case of a vibrating solid body which is also moving as a whole. For the velocity of any point in the solid equals the velocity of the centre of gravity plus the velocity of the point relatively to its centre of gravity. This latter velocity will involve $\dot{w}$, so that the 
square of the velocity and therefore the kinetic energy may involve $\dot{x} \dot{w}$.

3. Terms of the form

$$
(x \eta) \dot{x} \dot{\eta}
$$

cannot exist, for we can prove that they would involve the existence of a magnetizing force in a moving body depending upon the acceleration of the body. It would also require that the mechanical force exerted by a magnet should depend upon the rate of change of the magnetization. None of these effects have been observed.

4. Terms of the form

$$
(x \xi) \dot{x} \xi
$$

apparently do not exist, for they would require that the mechanical force exerted by a magnet should depend upon the rate of variation of the magnetic intensity, and this effect has not been observed.

5. Terms of the form

$$
\text { ( } y w) \dot{y} \dot{w} \text {. }
$$

If these terms existed it would be possible to develop electromotive forces by vibration, and these forces would depend upon the acceleration of the vibration and not merely upon the velocity; as these have not been observed we conclude that this term does not exist in the Lagrangian function of physical systems.

6. Terms of the form

$$
(y \eta) \dot{y} \dot{\eta} .
$$

If these terms existed there would be electromotive forces depending upon the rate of acceleration of the changes in the magnetic field.

They also indicate magnetic forces depending upon the rate of change of the current. As neither of these effects 
have been observed we conclude that terms of this form do not exist in the expression for the Lagrangian function.

7. Terms of the form

$$
(y \xi) \dot{y} \xi \text {. }
$$

Terms of this kind only involve the production of an electromotive force in a varying magnetic field, the electromotive force varying as the rate of change of the magnetic field. This is the well-known phenomenon of the production of an electromotive force round a circuit whenever the number of lines of magnetic force passing through it is changed.

As the term we are considering is the only one in the Lagrangian function which could give rise to an effect of this kind without also giving rise to other effects which have not been verified by experience, we conclude that this term does exist.

8. Terms of the form

$$
(w \eta) \dot{w} \dot{\eta} .
$$

If we take any molecular theory of magnetism, such as Ampère's, where the magnetic field depends upon the arrangement of the molecules of the body, we should rather expect this term to exist. The consequences of its existence have however not been detected by experiments.

If this term existed, then considering in the first place its effect upon the magnetic configuration we see that a vibrating body should produce magnetic effects depending upon the vibrations. Secondly, considering the effects of this term on the strain configuration we see that there should be a distorting force depending upon the rate of acceleration of the magnetic field. As neither of these effects have been observed there is no evidence of the existence of this term. 
9. Terms of the form

$$
(w \xi) \dot{w} \xi \text {. }
$$

These would involve the existence of distorting forces depending upon the rate of change of the magnetic field, and we have no evidence of any such effect.

Io. Terms of the form

$$
(\eta \xi) \dot{\eta} \xi \text {. }
$$

If we assume Ampère's hypothesis of molecule currents this term is of the same nature as the term $(x \xi) \dot{x} \xi$ which we discussed before, so that unless the properties of these molecular circuits differ essentially from those of finite size with which we are acquainted this term cannot exist.

2r. Summing up the results of the foregoing considerations, we arrive at the conclusion that the terms in the Lagrangian function which represent the kinetic energy depending upon the five classes of coordinates we are considering must be of one or other of the following types :

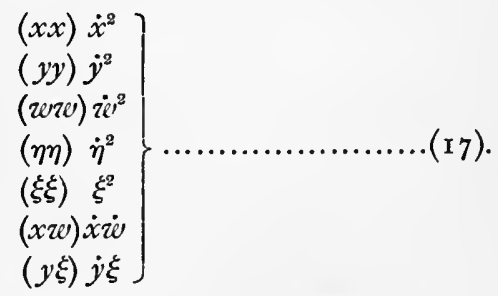

22. We might make a model with five degrees of freedom which would illustrate the connection between these phenomena which are fixed by coordinates of five types.

And if we arrange the model so that its configuration being defined by the five coordinates $x, y, w, \eta, \zeta$, only those terms which are in the expression ( $\mathrm{I} 7$ ) shall exist in 
the expression for its kinetic energy, and make the potential energy of the model corresponding to each coordinate analogous to that possessed by the physical system, then the working of this model will illustrate the interaction of phenomena in electricity, magnetism, elasticity \&c., and any phenomenon exhibited by the model will have its counterpart in the phenomena exhibited in these subjects.

When however we know the expression for the energy of such a model, there is no necessity to construct it in order to see how it will work, as we can deduce all the rules of working by the application of Lagrange's Equations. And from one point of view we may look upon the method we are using in this book as that of forming, not a model, but the expression for the Lagrangian function of a model every property of which must correspond to some actual physical phenomenon. 


\section{CHAPTER IV.}

\section{DISCUSSION OF THE TERMS IN THE LAGRANGIAN FUNCTION.}

23. WE must now proceed to examine the terms in the expression ( 17 ) more in detail, and find what coordinates enter into the various coefficients $(x x),(y y) \ldots$ When we have proved that these coefficients involve some particular coordinates we must go on to see what the physical consequences will be. In this way we shall be able to obtain many relations between the phenomena in electricity, magnetism and elasticity.

24. The first term we have to consider is $\{x x\} \dot{x}^{2}$, which corresponds to the expression for the ordinary kinetic energy of a system of bodies. We know that $\{x x\}$ may be a function of the geometrical coordinate typified by $x$, but we need not stop to consider the consequences of this as they are fully developed in treatises on the Dynamics of a System of Rigid Bodies.

Next $\{x x\}$ may involve the electrical coordinate $y$, for in a paper "On the Effects produced by the Motion of Electrified Bodies," Phil. Mag. Apr. I88ı, I have shown that the kinetic energy of a small sphere of mass $m$ charged 
with a quantity of electricity $e$ and moving with a velocity $v$ is

$$
\left\{\frac{\mathrm{I}}{2} m+\frac{2}{\mathrm{I} 5} \frac{\mu e^{2}}{a}\right\} v^{2} \ldots \ldots \ldots \ldots \ldots \ldots(\mathrm{I} 8),
$$

where $a$ is the radius of the sphere and $\mu$ the magnetic permeability of the dielectric surrounding it.

The existence in the kinetic energy of this term, which is due to the "displacement currents" started in the surrounding dielectric by the motion of the electrification on the sphere, shows that electricity behaves in some respects very much as if it had mass. For we see by the expression (I8) that the kinetic energy of an electrified sphere is the same as if the mass of the body had been increased by $4 \mu e^{2} / r_{5} a$.

Thus whenever a moving body receives a charge of electricity its velocity will be impulsively changed, for the momentum will remain constant, and as the apparent mass is suddenly increased the velocity must be impulsively diminished.

The apparent increase in mass cannot exceed a very small quantity because air or any other dielectric breaks down when the electric force gets very intense. If we take 75 as the intensity in electrostatic measure in C.G.S. units of the greatest electric force which a fairly thick layer of air can stand, which is the value given by Dr Macfarlane (Phil. Mag., Dec. r880), we have, since the electric force at the surface of the sphere must be less than 75 ,

$$
\frac{e}{K a^{2}}<75,
$$

$K$ being the specific inductive capacity of the medium.

So that the ratio of the increase in mass to the original mass, which by $(18)$ is equal to

$$
\frac{4}{15} \frac{\mu e^{2}}{a} / m \text {, }
$$


cannot exceed

and since in air

$$
\begin{gathered}
K \times 1500 \mu K a^{3} / m, \\
\mathbf{1} / \mu K=9 \times 10^{20}, \\
K=\mathbf{1},
\end{gathered}
$$

we see that the ratio cannot exceed

$$
\text { I. } 6 \times 10^{-18} a^{3} / m
$$

or about

$$
4 \times 10^{-19} / \rho \text {, }
$$

where $\rho$ is the mean density of the substance enclosed by the electrified surface.

Thus the alteration in mass, even if the mean density inside the surface is as small as that of air at the atmospheric pressure and $0^{\circ} \mathrm{C}$., is only about $5 \times 10^{-16}$ of the original mass, and is much too small to be observed.

Let us now consider the electrical effects of this term.

Let $Q$ be the electromotive force acting on the sphere. The energy of the system, using the same notation as before, is

$$
\left(\frac{1}{2} m+\frac{2}{15} \frac{\mu e^{2}}{a}\right) v^{2}+\frac{1}{2} \frac{e^{2}}{K a} .
$$

If $v$ be increased by $\delta v$ and $e$ by $\delta e$, the increment in the energy is

$$
\left(m+\frac{4}{\mathrm{I}_{5}} \frac{\mu e^{2}}{a}\right) v \delta v+\frac{4}{\mathrm{I}_{5}} \frac{\mu v^{2}}{a} e \delta e+\frac{e \delta e}{K a},
$$

and by the Conservation of Energy this must equal

so that

$$
Q \delta e
$$

$$
\left(m+\frac{4}{\mathrm{I}_{5}} \frac{\mu e^{2}}{a}\right) v \delta v+\frac{4}{\mathrm{I}_{5}} \frac{\mu v^{2}}{a} e \delta e+\frac{e \delta e}{K a}=Q \delta e \ldots \text { (19). }
$$

Since no mechanical force acts upon the system the momentum will be constant, so that

$$
\left(m+\frac{4}{\mathrm{I}_{5}} \frac{\mu e^{2}}{a}\right) \delta v+\frac{8}{\mathrm{1}_{5}} \frac{\mu e}{a} v \delta e=0 \ldots \ldots \ldots \ldots(20) .
$$

T. D. 
Eliminating $\delta v$ and $\delta e$ between equations (19) and (20) we find

$$
\frac{e}{K a}\left\{\mathrm{I}-\frac{4}{\mathrm{I} 5} \mu K v^{2}\right\}=Q \ldots \ldots \ldots \ldots \ldots(2 \mathrm{I}) .
$$

So that the capacity of the sphere is increased in the ratio of $\mathrm{I}$ to $\mathrm{I} / \mathrm{I}-\frac{4}{\mathrm{I} 5} \mu K v^{2}$, or since according to the Electromagnetic Theory of Light $\mu K=I / V^{2}$ where $V$ is the velocity of light through the dielectric, the capacity of the sphere is increased in the ratio of $I$ to $I / I-\frac{4}{I_{5}} \frac{v^{2}}{V^{y}}$. Thus the capacity of a condenser in motion will not be the same as that of the same condenser at rest, but as the difference depends on the square of the ratio of the velocity of the condenser to the velocity of light it will be exceedingly small.

If the earth does not carry the ether with it, a point on the earth's surface will be moving relatively to the ether, and the alterations in the velocity of such a point which occur during the day will produce a small diurnal variation in the capacities of condensers.

25. When we have two spheres of radius $a$ and $a^{\prime}$ moving with velocities $v$ and $v^{\prime}$ respectively the kinetic energy (see the paper on the "Effects produced by the Motion of Electrified Bodies," Phil. Mag., April, I881), assuming Maxwell's theory, is

$$
\left\{\left(\frac{1}{2} m+\frac{2}{15} \frac{\mu e^{2}}{a}\right) v^{2}+\left(\frac{1}{2} m^{\prime}+\frac{2}{15} \frac{\mu e^{\prime 2}}{a^{\prime}}\right) v^{\prime 2}+\frac{\mu e^{\prime} \cos \epsilon}{3 R} v v^{\prime}\right\}(22),
$$

where $R$ is the distance between the centres of the two spheres and $\epsilon$ the angle between their directions of motion; $m, m^{\prime}, e, e^{\prime}$ are respectively the masses and charges of the spheres. 
By expressing $v, v^{\prime}$ and $\cos \epsilon$ in terms of the coordinates of the centres of the spheres and their differential coefficients with respect to the time, we can, by using Lagrange's equations in the way explained in $\$ 20$, show that these terms require the existence of the following forces on the two spheres, $\dot{v}$ and $\dot{v}^{\prime}$ being the accelerations of the spheres respectively.

On the first sphere.

a. An attraction

$$
\frac{\mu c e^{\prime}}{3 R^{2}} v v^{\prime} \cos \epsilon
$$

along the line joining the centres of the spheres.

$\beta$. A force

$$
\frac{\mu e e^{\prime}}{3 R} \ddot{v}^{\prime}
$$

in the direction opposite to the acceleration of the second sphere.

$\gamma$. A force

$$
\frac{\mathrm{I}}{3} \mu c e^{\prime} v^{\prime} \frac{d}{d t}\left(\frac{\mathrm{I}}{R}\right)
$$

in the direction opposite to the direction of motion of the second sphere.

There are corresponding forces on the second sphere, and we see that unless the two spheres move with equal and uniform velocities in the same direction the forces on the two spheres are not equal and opposite. The sum of the momenta of the two spheres will not increase indefinitely however, since the sum of the actions and reactions is not constant but is a function of the accelerations.

We may easily prove that if $x, y, z$ are the coordinates of the centre of one sphere, $x^{\prime}, y^{\prime}, z^{\prime}$ those of the other, then

$$
\left\{m+\frac{4}{\mathrm{I}_{5}} \frac{\mu e^{2}}{a}+\frac{\mu e e^{\prime}}{3 R}\right\} \frac{d x}{d t}+\left\{m^{\prime}+\frac{4}{\mathrm{I}_{5}} \frac{\mu e^{\prime 2}}{a^{\prime}}+\frac{\mu}{3} R\right\} \frac{\left.e e^{\prime}\right\}}{d t}
$$


is constant, along with symmetrical expressions for the $y$ and $z$ coordinates.

26. Other electrical theories besides Maxwell's lead to the conclusion that the coefficient $\{x x\}$ is a function of the electrification of the system.

Thus according to Clausius' theory (Crelle, 82, p. 85) the forces between two small electrified bodies in motion are the same as if, using the same notation as before, there was the term

$$
e e^{\prime} v v^{\prime} \frac{\cos \epsilon}{R}-\frac{e e^{\prime}}{K R}
$$

in the expression for the Lagrangian function. The first of these is the same as the term we have just been considering.

'The forces which according to Weher's theory (Abhandlungen der Königlich Sächsischen Gesellschaft der Wissenschaften, I846, p. 2 I I. Maxwell's Electricity and Magnetism, and Edit. vol. II. $\$ 853$ ) exist between two electrified bodies in motion may easily be shown to be the same as those which would exist if in the Lagrangian function there was the term

$$
\frac{e e^{\prime}}{R}\left\{\frac{x-x^{\prime}}{R}\left(u-u^{\prime}\right)+\frac{y-y^{\prime}}{R}\left(v-v^{\prime}\right)+\frac{z-z^{\prime}}{R}\left(w-z c^{\prime}\right)\right\}^{2}-\frac{e e^{\prime}}{K R},
$$

where $x, y, z, x^{\prime}, y^{\prime}, z^{\prime}$ are the coordinates of the centres of the electrified bodies and $u, v, w, u^{\prime}, v^{\prime}, w^{\prime}$ the components of their velocities parallel to the axes of coordinates.

This term leads however to inadmissible results, as we can see by taking the simple case when the bodies are moving in the same straight line which we may take as the axis of $x$. In this case the term in the kinetic energy reduces to

or

$$
\begin{gathered}
\frac{e e^{\prime}}{R}\left(u-u^{\prime}\right)^{2} \\
\frac{e e^{\prime}}{R}\left(u^{2}-2 u u^{\prime}+u^{\prime 2}\right)
\end{gathered}
$$


so that the electrified bodies will behave as if their masses were in consequence of the electrification increased by $2 e e^{\prime} \mid R$ since the coefficients of $u^{2}$ and $\iota^{\prime 2}$ are each increased by half this amount. Hence if we take $e$ and $e^{\prime}$ of opposite signs and suppose the electrifications are great enough to make $2 e e^{\prime} \mid R$ greater than the masses of one or both of the bodies, then one of the bodies at least will behave as if its mass were negative. This is so contrary to experience that we conclude the theory cannot be right. This consequence of Weber's theory was first pointed out by v. Helmholtz (Wissenschaftliche Abhandlungen, r. p. 647).

The forces which according to Riemann's theory, given. in his posthumous work Schwere, Elektricität und Magnetismus, p. 326, exist between two moving electrified bodies may easily be shown to be the same as those which would exist if there were the term

$$
\frac{e e^{\prime}}{R}\left\{\left(u-u^{\prime}\right)^{2}+\left(v-v^{\prime}\right)^{2}+\left(w-w^{\prime}\right)^{2}\right\}-\frac{e e^{\prime}}{K R}
$$

in the expression for the Lagrangian function. We can easily see that this theory is open to the same objection as Weber's, that is, it would make an electrified body behave in some cases as if its mass were negative.

27. If we regard the expression for the kinetic energy from the point of view of its bearing on electrical phenomena we shall see that it shows that if we connect the terminals of a battery to two spheres made of conducting material, the quantity of electricity on the spheres will depend upon their velocities.

We see from the expression (22) for the kinetic energy of a moving conductor that if we have a number of conductors moving.about in the electric field there will be a positive term in the Lagrangian function depending upon the square of the electrification. And the same is true to a smaller 
extent if the moving bodies are not conductors but substances whose specific inductive capacity differs from that of the surrounding medium. This is equivalent to a decrease in the potential energy produced by a given electrification, since an increase in the potential energy corresponds to a decrease in the Lagrangian function. Thus the presence of the moving conductors is equivalent to a diminution in the stiffness of the dielectric with respect to alterations in its state of electrification. And therefore the speed with which electrical oscillations are propagated across any medium will be diminished by the presence of molecules moving about in it; the diminution being proportional to the square of the ratio of the velocity of the molecules to the velocity with which light is propagated across the medium. Thus if the electromagnetic theory of light is true the result we have been discussing has an important bearing on the effect of the molecules of matter on the rate of propagation of light.

28. We can see that $\{x x\}$ may be a function of the strain coordinates, for let us take the case when $\{x x\}$ is the moment of inertia of a bar about an axis through its centre : then it is evident if the bar be compressed in the middle and pulled out at the ends that the moment of inertia will be less than if the bar were unstrained, for the effect of the strain has practically been to bring the matter forming the bar nearer to the axis. Thus the moment of inertia and therefore $\{x x\}$ may depend upon the strain coordinates.

These coordinates will in general only enter $\{x x\}$ through the expression for the alteration in the density of the strained body, i.e. through

$$
\frac{d \alpha}{d x}+\frac{d \beta}{d y}+\frac{d \gamma}{d z} \ldots \ldots \ldots \ldots \ldots \ldots(23),
$$

and this will only enter $\{x x\}$ linearly. 
If we form the equations of elasticity by using Hamilton's principle

$$
\delta \int_{t_{0}}^{t_{1}}(T-V) d t=0
$$

we shall easily find that the presence of $(23)$ in $\{x x\}$ leads to the introduction of the so-called "centrifugal forces" into the equation of elasticity for a rotating elastic solid. This however we shall leave as an exercise for the reader.

29. Let us now consider that part of the Lagrangian function which depends upon the velocities of the electrical coordinates, i.e. the part denoted by

$$
\frac{1}{2}\left\{\left(y_{1} y_{1}\right) \dot{y}_{1}^{2}+2\left(y_{1} y_{2}\right) \dot{y}_{1} \dot{y}_{2}+\ldots\right\} \text {. }
$$

Let us take the case of two conducting circuits whose electrical configuration is fixed by the coordinates $y_{1}, y_{2}$, where $\dot{y}_{1}, \dot{y}_{g}$ are the currents flowing through the circuits respectively.

This part of the Lagrangian function may in this case be conveniently written

$$
\frac{1}{2}\left(L \dot{y}_{1}^{2}+2 M \dot{y}_{1} \dot{y}_{2}+N \dot{y}_{2}^{2}\right) .
$$

Now we can fix the geometrical configuration of the two circuits if we have coordinates which can fix the position of the centre of gravity and the shape and situation of the first circuit, the shape of the second circuit and its position relatively to the first.

Let us denote by $x_{2}-x_{1}$ any coordinate which helps to fix the position of one circuit relatively to the other, and by $\xi_{1}, \xi_{2}$ coordinates helping to fix the shape of the first and second circuits respectively.

It is evident that the kinetic energy must be expressible in terms of these coordinates, for the only coordinates necessary to fix the system which we have omitted are those fixing the centre of gravity and situation of the first circuit, and 
since a motion of the whole system as a rigid body through space cannot alter this part of the kinetic energy of the system, the expression for the kinetic energy cannot involve these coordinates.

If we write for a moment $x$ instead of $x_{2}-x_{1}$ (a coordinate helping to fix the position of one circuit relatively to the other) then by Lagrange's Equations we see that these terms in the kinetic energy correspond to the existence of a force tending to increase $x$ equal to

$$
\frac{1}{2} \frac{d L}{d x} \dot{y}_{1}{ }^{2}+\frac{d M}{d x} \dot{y}_{1} \dot{y}_{2}+\frac{1}{2} \frac{d N}{d x} \dot{j}_{2}{ }^{2} \ldots \ldots . .(24) .
$$

We see from this expression that $d L / d x$, and $d N / d x$ must vanish, otherwise there would be a force between the two circuits even though the current in one of them vanished. The quantities $L$ and $N$ are by definition the coefficients of self-induction of the two circuits, and hence we see that the coefficient of the self-induction of a circuit is independent of the position of other circuits in its neighbourhood and is therefore the same as if these circuits were removed ${ }^{1}$.

By (I6) the force tending to increase $x$ is

$$
\frac{d M}{d x} \dot{y}_{1} \dot{y}_{2}
$$

that is there is a force between the two circuits proportional to the product of the currents flowing through them, and also to the differential coefficient with respect to the coordinate along which the force is reckoned of a function which does not involve the electrical coordinates. This corresponds exactly to the mechanical forces which are actually observed

1 This is quite consistent with the apparent diminution in the selfinduction caused by a neighbouring circuit when an alternating current is used. 
between the circuits, and a little consideration will show that these forces could not arise from any other terms in the Lagrangian function. Thus the consideration of the mechanical forces which two circuits carrying currents are known to exert upon each other proves that the term $M \dot{y}_{1} \dot{y}_{2}$ exists in the expression for the Lagrangian function.

Let us now go on to consider the effect of these terms on the electrical configuration of the two circuits.

By Lagrange's Equation for the coordinate $y_{1}$ we have

$$
\frac{d}{d t} \frac{d L^{\prime}}{d \dot{y}_{1}}-\frac{d L^{\prime}}{d y_{1}}=Y_{1} \ldots \ldots \ldots \ldots \ldots(25),
$$

where $Y_{1}$ is the external electromotive force tending to increase $y_{1}$. Now as we shall prove directly $d L^{\prime} / d y_{1}=0$, so that the effects on the electrical configuration of the first circuit, arising from the term

$$
\frac{1}{2}\left(L \dot{y}_{1}{ }^{2}+2 M \dot{y}_{1} \dot{y}_{2}+N \dot{y}_{2}{ }^{2}\right)
$$

are the same as would be produced by an external electromotive force tending to increase $y_{1}$ equal to

$$
-\frac{d}{d t}\left(L \dot{y}_{1}+M \dot{y}_{2}\right) \ldots \ldots \ldots \ldots \ldots \ldots \ldots(26) .
$$

Thus if any of the four quantities $L, M, \dot{y}_{1}, \dot{y}_{2}$ vary in value there is an electromotive force acting round the circuit through which the current $\dot{y}_{1}$ flows. And the expression (26) gives the E. M. F. produced either by the motion of neighbouring circuits conveying currents or by alterations in the magnitudes of the currents flowing through the circuits.

This example is given in Maxwell's Electricity and Magnetism, vol. II. part IV. chapter vi., and it is one which illustrates the power of the dynamical method very well. The existence of the mechanical force shows that there is 
the term

$$
M \dot{y}_{1} \dot{y}_{2},
$$

in the expression for the Lagrangian function and then the law of the induction of currents follows at once by the application of Lagrange's Equations.

The problem we have just been considering is dynamically equivalent to finding the equations of motion of a particle with two degrees of freedom when under the action of any forces. We know that these cannot be deduced by the aid of the principle of the Conservation of Energy alone, for to take the simplest case of all, that in which no forces act upon the particle, the principle of the Conservation of Energy is satisfied if the velocity is constant whether the particle moves in a straight line or not. From this analogy we see that when we have two circuits the principle of the Conservation of Energy is not sufficient to deduce the equations of motion, and that some other principle must be assumed implicitly in those proofs which profess to deduce these equations by means of the Conservation of Energy alone.

30. There is no experimental evidence to show that $\{y y\}$ is a function of the electrical coodinates $y$, and it certainly is not when the electrical systems consist of a series of conducting circuits, for if it were the coefficients of self and mutual induction would depend upon the length of time the currents had been flowing through the circuits. And in any case it would require the existence of electromotive forces which would not be reversed if the direction of all the electric displacements in the field were reversed.

31. Similar reasoning will show that $\{y y\}$ cannot be a function of the magnetic coordinates, for if it were there would be magnetic forces produced by electric currents 
which would not be reversed if the directions of all the currents in the field were reversed.

32. We must now consider whether $\{y y\}$ is a function of the strain coordinates or not. If it is then the coefficients of self and mutual induction of a number of circuits must depend upon the state of strain of the wires forming the circuits. This result though not impossible has never been detected, and it is contrary to Ampere's hypothesis that the force exerted by a current depends only upon its strength and position and not upon the nature or state of the material through which it flows.

Then again, if we consider what the effect on the elastic properties of the substance would be if $\{y y\}$ were a function of the strain coordinates, we see at once that it would indicate that the elastic properties of a wire would be altered while an electric current was passing through it.

The evidence of various experimenters on this point is somewhat conflicting. Both Wertheim (Ann. de Chim. et de Phys. [3] г 2, p. 6ro, Wiedemann's Elektricität, ir. p. 403) and Tomlinson have observed that the elasticity of a wire is diminished when a current passes through it and that this diminution is not due to the heat generated by the current. Streintz (Wien. Ber. [2] 67, p. 323, Wiedemann's Elektricität, II. p. 404) on the other hand was unable to detect any such effect.

But even if this effect were indisputably established it would not prove rigorously that $\{y y\}$ is a function of the strain coordinates, for as we shall endeavour to show when we consider electrical resistance this effect might have been due to another cause.

To sum up we see that $\{y y\}$ is a function of the geometrical coordinates but not of the electric or magnetic ones and probably not of the strain ones. 
33. We shall now consider the part of the Lagrangian function which depends upon the magnetic coordinates and which does not involve the velocities of the geometrical, electrical or strain coordinates. Thus the terms we are about to consider in the Lagrangian function of unit volume of a substance are those we have denoted by

$$
\frac{1}{2}\{\xi \xi\} \xi^{2}+\frac{1}{2}\{\xi\} \xi+\frac{1}{2}(\eta \eta) \dot{\eta}^{2}
$$

we may have in addition to these terms arising from the potential energy.

In order to begin with as simple a case as possible let us suppose that all the magnetic changes take place indefinitely slowly; in this case we may neglect the term

$$
\frac{1}{2}(\eta \eta) \dot{\eta}^{2}
$$

and confine our attention to the terms

$$
\frac{1}{2}\{\xi \xi\} \xi^{2}+\frac{1}{2}(\xi) \xi
$$

or as it is more convenient to write them

$$
\frac{1}{2} A \eta^{2} \xi^{2}+\frac{1}{2}\{\xi\} \xi
$$

Let us take first the case when the magnetization is parallel to one of the axes, $x$ for example, and let us denote the magnetic force parallel to this direction by $H$ and the intensity of magnetization by $I$, where by definition

$$
I=\eta \xi \text {.. }
$$

The investigation in $\S 389$ of Maxwell's Electricity and Magnetism shows that if we suppose that all the energy in the magnetic field is resident in the magnets, there is in the Lagrangian function for unit volume of a magnet the term

\section{$H I$.}

The result of this investigation is stated in the Electricity 
and Magnetism to be that the potential energy of unit volume of the magnet is

$$
-H I \text {, }
$$

but we have seen in $\S 9$ that the question whether energy determined in this manner is kinetic or potential is really left unsettled: what is actually proved is that a certain term exists. in the Lagrangian function.

If we suppose that the energy is distributed throughout the whole of the magnetic field, including unmagnetized substances as well as magnets, then the investigation in $\S 635$ of the Electricity and Magnetism shows that the Lagrangian function of unit volume anywhere in the magnetic field contains the term

$$
\frac{\mathrm{I}}{8 \pi} H B,
$$

where $B$ is the magnetic induction.

These two ways of regarding the energy in the magnetic field lead to identical results; and as we shall for the present confine our attention to the magnetized substances we shall find it more convenient to adopt the first method of looking at the question.

We have seen that the Lagrangian function for unit volume of a magnet contains the term

or in our notation

$$
H I \text {, }
$$

$$
H \xi \xi ;
$$

and this is the term we previously denoted by

$$
\frac{1}{2}\{\xi\} \xi \text {. }
$$

Since the magnetic changes are supposed to take place indefinitely slowly, Lagrange's equation for the $\eta$ coordinate reduces to

$$
\frac{d L^{\prime}}{d \eta}=0 \ldots \ldots \ldots \ldots \ldots \ldots \ldots . . . . .(29) .
$$


Applying this to the expression (27) and substituting $H \eta \xi$ for $\frac{1}{2}\{\xi\} \xi$ we get

$$
\frac{1}{2} \frac{d}{d \eta}\left(A \eta^{2} \xi^{2}\right)+H \xi=0 \ldots \ldots \ldots \ldots . .(30),
$$

and since $\xi$ is supposed to remain constant and therefore

this may be written

$$
\xi d \eta=d I
$$

$$
I \frac{d}{d I^{2}}\left(A I^{2}\right)+H=0 \ldots \ldots \ldots \ldots . .(3 \mathrm{I}) .
$$

So that if $k$ be the coefficient of magnetic induction and defined by the equation

we have by (3I)

$$
I=k H
$$

$$
\frac{\mathrm{I}}{k}=-\frac{d}{d I^{2}}\left(A I^{2}\right) \ldots \ldots \ldots \ldots \ldots \ldots\left(3^{2}\right),
$$

and therefore

$$
\begin{aligned}
A I^{2} & =-\int \frac{1}{k} d I^{2} \\
& =-2 \int H d I .
\end{aligned}
$$

If we know the way in which $I$ varies with $H$ we could by this equation express $A$ as a function of $I$. The relation between $I$ and $H$ is however in general so complicated that there seems but little advantage to be gained by taking some empirical formula which connects the two and determining $A$ by its help.

For small values of $H$, Lord Rayleigh (Phil. Mag. 23, p. 225,1887 ) has shown that $I / H$ is constant, so by equation (33) $A$ in this case is also constant.

34. The mechanical force parallel to the axis of $x$ acting on unit volume of the magnet is

$$
\frac{d L^{\prime}}{d x} \text {. }
$$


The only quantity in the terms we are considering which involves $x$ explicitly is $H$, so that $d L^{\prime} / d x$ reduces to

or

$$
\begin{gathered}
\eta \xi \frac{d H}{d x} \\
I \frac{d H}{d x}
\end{gathered}
$$

and this is the mechanical force parallel to the axis of $x$ acting on unit volume of the magnet. This expression may also be written

$$
\frac{1}{2} k \frac{d H^{2}}{d x}
$$

with similar expressions for the components parallel to the axes of $y$ and $z$.

These are the same expressions for this force as those given in Maxwell's Electricity and Magnetism, vol. 11. p. 70, the consequences of which are as is well known in harmony with Faraday's investigations on the way in which paramagnetic and diamagnetic bodies move when placed in a variable magnetic field.

35. We have just investigated the mechanical forces produced by a magnetic field; we shall now proceed to investigate some of the stresses produced by it.

Let us take the case of a cylindrical bar of soft iron whose axis coincides with the axis of $x$, and suppose that it is magnetized along its axis. Let $e, f, g$ be the dilatations of the bar parallel to the axes of $x, y, z$ respectively. We shall at present assume that there is no torsion in the bar. We shall suppose that the changes in the strains take place so slowly that we may neglect the kinetic energy arising from them.

The potential energy due to these strains is

$$
\frac{1}{2} m\{e+f+g\}^{2}+\frac{1}{2} n\left\{e^{2}+f^{2}+g^{2}-2 e f-2 e g-2 f g\right\} \text {, }
$$


where $n$ is the coefficient of rigidity and $m-n / 3$ the modulus of compression.

Thus the terms in the Lagrangian function involving the magnetic and strain coordinates are

$$
\frac{1}{2} A \eta^{2} \xi^{2}+H \eta \xi-\frac{1}{2} m(e+f+g)^{2}-\frac{1}{2} n\left(e^{2}+f^{2}+g^{2}-2 e f-2 e r\right.
$$

neglecting those depending on the rate of variation of these quantities which rate we shall assume to be indefinitely small.

The experiments of Villari and Sir William Thomson (Wiedemann's Elektricität, III. p. jor) have shown that $k$ depends upon the strain in the magnet, hence by equation (32) $A$ will be a function of the strains. We shall proceed to investigate the stresses which arise in consequence of this. Using the Hamiltonian principle

$$
\delta \int_{t_{0}}^{t_{1}} L d t=0
$$

and substituting $d \alpha / d x, d \beta / d y, d \gamma / d z$ for $e, f, g$ respectively, we get the following equations by equating to zero the variation caused by changing $\alpha$ into $a+\delta \alpha$

inside the bar,

$$
\frac{d L}{d x}-\frac{d}{d x} \frac{d L}{d e}=0 ;
$$

at the boundary.

$$
\frac{d L}{d e}=0
$$

By equating to zero the variation caused by changing $\beta$ into $\beta+\delta \beta$ we get

inside the bar,

$$
\frac{d L}{d y}-\frac{d}{d y} \frac{d L}{d f}=0
$$

$$
\frac{d L}{d f}=0
$$

at the boundary. 
And by equating to zero the variation caused by changing $\gamma$ into $\gamma+\delta \gamma$ we get

$$
\frac{d L}{d z}-\frac{d}{d z} \frac{d L}{d r}=0
$$

inside the bar;

$$
\frac{d L}{d y}=0
$$

at the boundary.

The first and second terms in the equations (35), (37) and (39) may conveniently be considered separately. Since $H$ is the only quantity in the expression for $I$, which can involve the coordinates $x, y$, or $z$ explicitly the terms

$$
\frac{d L}{d x}, \frac{d L}{d y}, \frac{d L}{d z}
$$

reduce to

or

$$
\eta \xi \frac{d H}{d x}, \eta \xi \frac{d H}{d y}, \eta \xi \frac{d H}{d z}
$$

$$
k H \frac{d H}{d x}, k H \frac{d H}{d y}, k H \frac{d H}{d z}
$$

respectively.

These are the expressions for the components of the mechanical force acting on the body, and it is shown in Maxwell's Electricity and Magnetism, $\$ 642$, that this distribution of force would strain the body in the same way as "a hydrostatic pressure $H^{2} / 8 \pi$ combined with a tension $B H / 4 \pi$ along the lines of force," $B$ being the magnetic induction. Thus we may suppose that the strains arising from these terms are known. If $e, f, g$ are the strains due to the second term in equations (35) (37) and (39), we have 


$$
\left.\begin{array}{l}
\eta^{\mathrm{e}} \xi^{2} \frac{d A}{d e}-m(e+f+g)-n(e-f-g)=0 \\
\eta^{2} \xi^{2} \frac{d A}{d f}-m(e+f+g)-n(f-e-g)=0 \\
\eta^{2} \xi^{2} \frac{d A}{d g}-m(e+f+g)-n(g-e-f)=0
\end{array}\right\} \ldots(4 \mathrm{I}) .
$$

Solving these equations and putting $\eta \xi=I$ we get

$$
\left.\begin{array}{l}
2 n e=\frac{2 m}{3^{m-n}} \frac{d}{d e}\left(A I^{2}\right)-\frac{m-n}{3^{m-n}}\left\{\frac{d}{d f}\left(A I^{2}\right)+\frac{d}{d g}\left(A I^{2}\right)\right\} \\
2 n f=\frac{2 m}{3^{m-n} n} \frac{d}{d f}\left(A I^{2}\right)-\frac{m-n}{3^{m-n}}\left\{\frac{d}{d e}\left(A I^{2}\right)+\frac{d}{d g}\left(A I^{2}\right)\right\} \\
2 n g=\frac{2 m}{3^{m-n}} \frac{d}{d g}\left(A I^{2}\right)-\frac{m-n}{3^{m-n}}\left\{\frac{d}{d e}\left(A I^{2}\right)+\frac{d}{d f}\left(A I^{2}\right)\right\}
\end{array}\right\}
$$

If the magnet is symmetrical about its axis we have

and

$$
\begin{aligned}
f & =g \\
\frac{d}{d f}\left(A I^{2}\right) & =\frac{d}{d y}\left(A I^{2}\right) .
\end{aligned}
$$

So that equations $\left(4^{2}\right)$ reduce to

$$
\left.\begin{array}{c}
n e=\frac{m}{3^{m-n}} \frac{d}{d e}\left(A I^{2}\right)-\frac{m-n}{3^{m-n}} \frac{d}{d f}\left(A I^{2}\right) \\
2 n f=-\frac{m-n}{3^{m-n}} \frac{d}{d e}\left(A I^{2}\right)+\frac{m+n}{3^{m-n}} \frac{d}{d f}\left(A I^{2}\right)
\end{array}\right\}
$$

The dilatation $e+2 f$ is equal to

$$
\frac{\mathrm{I}}{3^{m-n}-n}\left\{\frac{d}{d e}\left(A I^{2}\right)+2 \frac{d}{d f}\left(A I^{2}\right)\right\} \ldots . . . .(44) .
$$

Differentiating equations (43) with regard to $I^{2}$, we get approximately since $\frac{d}{d e^{2}}\left(A I^{2}\right)$ and $\frac{d^{2}}{d e d f}\left(A I^{2}\right)$ must be small compared with $m$ or $n$, or the changes in the elasticity caused by magnetization would not be so small as to have escaped detection, 


$$
\left.\begin{array}{rl}
n \frac{d e}{d I^{2}} & =\frac{m}{3^{m-n}} \frac{d}{d e} \frac{d}{d I^{2}}\left(A I^{2}\right)-\frac{m-n}{3 m-n} \frac{d}{d f} \frac{d}{d I^{3}}\left(A I^{\varepsilon}\right) \\
2 n \frac{d f}{d I^{2}} & =-\frac{m-n}{3^{m-n}} \frac{d}{d e} \frac{d}{d I^{2}}\left(A I^{2}\right)+\frac{m+n}{3 m-n} \frac{d}{d f} \frac{d}{d I^{2}}\left(A I^{2}\right)
\end{array}\right\}
$$

Now by equation (32)

$$
\frac{d}{d I^{2}}\left(A I^{2}\right)=-\frac{\mathrm{I}}{k} \text {. }
$$

So that these equations become

$$
\left.\begin{array}{c}
n \frac{d e}{d I^{2}}=\frac{m}{3 m-n} \frac{\mathbf{I}}{k^{2}} \frac{d k}{d e}-\frac{m-n}{3^{m-n}} \frac{\mathrm{I}}{k^{2}} \frac{d k}{d f} \\
2 n \frac{d f}{d I^{2}}=-\frac{m-n}{3^{m-n}} \frac{\mathrm{I}}{k^{2}} \frac{d k}{d e}+\frac{m+n}{3^{m-n}} \frac{\mathrm{I}}{k^{2}} \frac{\frac{d k}{d f}}{\frac{m}{d 4}}
\end{array}\right\} \ldots(4)
$$

Now if the coefficient of magnetization depends upon the strains, the intensity of magnetization of the bar when under the action of a constant magnetizing force will be altered by strain, and in order to compare the formulae with the results of experiments we shall find it more convenient to express $d e / d I^{2}, d f / d I^{3}$ in terms of the changes which take place in the intensity of magnetization when the bar is stretched rather than in terms of $d k / d e$ and $d k / d f$.

We have

$$
I=k H \text {, }
$$

so that when $H$ is supposed to be constant

$$
\frac{d I}{d e}=H \frac{d k}{d e}+H \frac{d k}{d I} \frac{d I}{d e}
$$

and equations (46) may be written

$$
\left.\begin{array}{rl}
n \frac{d e}{d I^{2}} & =\left(\mathrm{I}-H \frac{d k}{d I}\right)\left(\frac{m}{3 m-n} \frac{\mathrm{I}}{k I} \frac{d I}{d e}-\frac{m-n}{3^{m-n}} \frac{\mathrm{I}}{k I} \frac{d I}{d f}\right) \\
2 n \frac{d f}{d I^{2}} & =\left(\mathrm{I}-H \frac{d k}{d I}\right)\left(-\frac{m-n}{3 m-n} \frac{\mathrm{I}}{k I} \frac{d I}{d e}+\frac{m+n}{3^{m-n}} \frac{\mathrm{I}}{k I} \frac{d I}{d f}\right)
\end{array}\right\}
$$

These expressions give the strains which result from the dependence of the intensity of magnetization on the state 
of strain of the magnetized body. In addition there are the strains arising from Maxwell's distribution of stress. Kirchhoff (Wied. Ann. xxiv. p. 52, xxv. p. 601) has investigated the effect of this on a small soft iron sphere placed in a uniform magnetic field and has shown that it would produce an elongation of the sphere along the lines of force and a contraction at right angles to them. We may therefore assume that in general this distribution of stress causes an expansion of the magnet in the direction of the lines of force and a contraction in all directions perpendicular to this.

The expressions for the strains in a magnetizable substance placed in the magnetic field have also been investigated by v. Helmholtz (Wied. Ann. xirl. p. 385 ). The object of the investigations of v. Helmholtz and Kirchhoff was rather different from that of Maxwell. Maxwell's object was to show that his distribution of stress would produce the same forces between magnetized bodies as those which are observed in the magnetic field, while v. Helmholtz and Kirchhoff's object was to show that it follows from the principle of the Conservation of Energy that, whatever theory of electricity and magnetism we assume, the bodies in the electric or magnetic field must be strained as if they were acted upon by a certain distribution of stress which in the simplest case is the same as that given by Maxwell.

We have in addition to the strain produced by these stresses, the strains depending upon the alteration of the intensity of magnetization with stress along and perpendicular to the lines of force.

The effect of stress along the lines of force on the magnetization of iron has been investigated by Villari (Pogg. Ann. 126, p. 87, 1868) and Sir William Thomson (Proc. Roy. Soc. 27, p. 439, 1878); both these physicists found that 
the intensity of magnetization was increased by stretching when the magnetizing force was small, but that when the magnetization exceeds about 10 when measured in C.G.S. units the intensity of magnetization is diminished by stretching.

Sir William Thomson also investigated the effect of stress at right angles to the lines of magnetic force on the intensity of magnetization and found that this was in general opposite to that of tension along the lines of force, so that for small values of the magnetizing force extension at right angles to the lines of force diminishes the magnetization, while for larger values of this force it increases it. The critical value of the force in this case however is higher than that for tension along the lines of force.

Thus, except when the magnetizing force is between the critical values, $d I / d c$ and $d I / d f$ have opposite signs, hence we see by equation (48) that except in this case, since Prof. Ewing's measurements show that $I I d k / d I$ is always less than unity,

$$
\frac{d e}{d l^{2}} \text { and } \frac{d I}{d e}
$$

have the same sign, and

$$
\frac{d f}{d I^{2}} \text { and } \frac{d I}{d e}
$$

have opposite signs.

Now $d I / d e$ is positive or negative according as the magnetizing force is less or greater than the critical value, so that when the magnetizing force is less than the critical value the extension we are investigating will increase with the magnetic force, but when the magnetizing force is greater than this value the extension will diminish as the force increases.

As we mentioned before the strain produced by Maxwell's distribution of stress, which is the other cause tending to 
strain the body, has been shown by Kirchhoff to produce an expansion along the lines of force and a contraction at right angles to them. Thus when the magnetizing force is less than the critical value this strain and the strain we have just investigated act in the same way, but when the force is greater they act in opposite directions.

Joule's investigations (Phil. Mag. 30, pp. 76, 225, 1847) prove that the length of an iron bar increases when it is magnetized and as far as the experiments went the increase in the length was proportional to the square of the magnetizing force. Mr Shelford Bidwell (Proc. Roy. Soc. XL. p. 109) however has lately shown that when the magnetizing force is very large the magnet shortens as the magnetizing force increases.

Comparing these experimental results with our theoretical conclusions we see that they are in accordance when the magnetizing force is small, and that when the magnetizing force is large they indicate that the strains due to the same cause as that which causes the intensity of magnetization to alter with strains are more powerful than those arising from Maxwell's distribution of stress. Prof. Ewing's experiments on the effect of strain on magnetization ("Experimental Researches in Magnetism," Phil. Trans. 1885, part 11. p. $5^{85}$ ) would seem to show that this must be the case. For Kirchhoff (Wiedemann's Annalen, xxv. p. 601) has shown that the greatest increase in length which Maxwell's stresses can produce in a soft iron sphere whose radius is $R$, placed in a uniform magnetic field where the force at an infinite distance from the sphere is $H$, is

$$
\frac{\mathrm{I} 53}{\mathrm{I} 76 \pi} \frac{H^{2}}{E} R,
$$

where $E$ is one of the constants of elasticity for soft iron and is equal in the C.G.S. system of units to $1.8 \times 10^{12}$. 
Thus in this case supposing $k$ to be constant we have

$$
\begin{array}{r}
\frac{d e}{d I^{g}}=\frac{\mathrm{I} 53}{\mathrm{I} 76 \pi k^{\mathrm{g}}} \frac{\mathrm{I}}{\mathrm{I} \cdot 8 \times 10^{19}} \\
=\frac{\mathbf{r} 5 \times \mathrm{IO}^{-13}}{k^{2}} \ldots
\end{array}
$$

Now according to Prof. Fwing's experiments the intensity of magnetization of a soft iron wire which was represented by 18I when there was no load was increased to 237 when the wire was loaded with a kilogramme, so that in this case

$$
\frac{\delta I}{I}=\frac{\mathrm{I}}{3} \ldots \text { nearly........... (50). }
$$

The diameter of the wire was such that the load of a kilogramme corresponded to a stress of about $2 \times 10^{8}$ per square centimetre in C.G.s. units, so that if $q$ be Young's modulus for the wire and $\delta e$ the extension produced by the load

$$
q \delta e=2 \times 10^{8}:
$$

for wrought iron $q / n$ is about 2.5 , so that

$$
n \delta e=8 \times 10^{\tau}
$$

and therefore by (49)

$$
\frac{\mathrm{I}}{n I} \frac{d I}{d e}=\frac{\mathrm{I}}{2 \cdot 4 \times 10^{8}}
$$

so that by equation (48) if $e$ be the elongation due to the magnetization

$$
\frac{d e}{d I^{2}}>\frac{\mathrm{I}}{7 \times 10^{8}} \frac{\mathrm{I}}{k} \ldots \ldots \ldots \ldots \ldots(5 \mathrm{I}) .
$$

Comparing this with (49) we see that the part of $d e / d I^{2}$ due to the cause we are now considering is very much greater than that due to Maxwell's distribution of stress. The value of $d I / d e$ is probably exceptionally large in this case, and near the critical value it is doubtless very much less, so that in this case it is conceivable that the effect of the Maxwell 
stress may be comparable with that due to the alteration of intensity of magnetization with strain.

Since the Maxwell effect is in general so small compared with the other we should expect the critical value of the magnetizing force to be approximately the same as the value of the force when the extension is a minimum; it is however much less. There seems however to be reason to think that the critical value when the magnet is free from strain has been very much underestimated. Indeed Prof. Ewing (loc. cit.) expresses his opinion that "if we deal only with very small stresses it is doubtful whether any reversal of the positive effect of stress would be reached even at the highest obtainable value of the magnetization." By the positive effect of stress Prof. Ewing means an increase of magnetization with an increase of stress, the magnetizing force remaining constant.

Bidwell's discovery that $d e / d I^{\mathrm{g}}$ is negative when the magnetization exceeds a certain value, in conjunction with the theoretical results we have been investigating in this paragraph, shows that when the magnetization reaches this value the positive effects of stress must be reversed. The magnet in this case however is not free from stresses as it is acted on by those called into play by the magnetization.

36. If the dilatation in volume $e+2 f$ be denoted by $\delta$, then the part of $\delta$ due to the same cause as that which makes the intensity of magnetization depend upon strain is by (48) given by the equation

$$
\frac{d \delta}{d I^{2}}=\frac{\mathbf{I}}{3^{m}-n} \frac{\mathrm{I}}{k I}\left(\frac{d I}{d e}+2 \frac{d I}{d f}\right)\left(\mathrm{I}-H \frac{d k}{d I}\right) \ldots(52) .
$$

Joule's experiments show that the dilatation in the volume if it exists at all must be very small compared with the elongation, as he was not able to detect it though his 
apparatus would have enabled him to do so if it had amounted to one part in 4500000 . Hence as the greater part of the strain is that given by equation (48)

$$
\frac{d I}{d e}+2 \frac{d I}{d f}
$$

must be small, so that $d I / d e$ and $d I / d f$ must have opposite signs except when they are very small. This agrees with the results of Sir William Thomson's experiments on the effects of traction along and perpendicular to the lines of force on the intensity of magnetization; as, except in the neighbourhood of the critical magnetic forces when $d I / d e$ and $d I / d f$ are both small, traction along and perpendicular to the lines of force produced opposite results.

If we assume that Joule's experiments prove that there is no change in volume then by equation (52)

$$
\frac{d I}{d e}+2 \frac{d I}{d f}=0
$$

and equation $(48)$ reduces to

$$
2 n \frac{d e}{d I^{2}}=\frac{\mathrm{I}}{k I} \frac{d I}{d e}\left(\mathrm{I}-H \frac{d k}{d I}\right)^{*} \text {. }
$$

37. The critical value of the intensity of magnetization, i.e. the intensity when the magnetization is neither increased nor decreased by a small strain will, since by $\left(3^{2}\right)$ and (47)

$$
\frac{\mathrm{I}}{k I}\left(\mathrm{I}-H \frac{d k}{d I}\right) \frac{d I}{d e}=\frac{d}{d e} \frac{d}{d I^{2}}\left(A I^{2}\right) \ldots \ldots \ldots \ldots(54) ;
$$

be given by the equation

$$
\frac{d}{d e} \frac{d}{d I^{2}}\left(A I^{2}\right)=0
$$

* In my paper on "Some Applications of Dynamics to Plysical Phenomena," Part 1. Phil. Trans. Part II. 1885 this equation has the wrong sign, which was carried down from equation $(51)$ in the same paper. 
The experiments of Sir William Thomson and Prof. Ewing have shown that the critical value of $I$ depends upon the state of strain. Hence we see by means of equation (55) that $d A / d e$ must be a function of $e$, so that if $A$ be expanded in powers of $e$ it must contain powers about the first. We may therefore write

$$
A=\alpha+\beta e+\gamma e^{\varepsilon}+\delta e^{3}+\ldots
$$

Now if the term $\gamma e^{2}$ exists, the coefficient of $e^{\varepsilon}$ in the Lagrangian function will contain the term $\frac{1}{2} \gamma I^{2}$ and so will involve the state of magnetization of the body. The coefficients of elasticity however are linear functions of the coefficient of $e^{2}$ in the Lagrangian function so that if this latter quantity depends upon the state of magnetization, the coefficients of elasticity will do the same. We conclude therefore that the elasticity of an iron bar must be altered by magnetization. This effect does not seem to have been observed.

If in the expression for $A$ we neglect powers above the second we have

$$
\frac{d A}{d e}=\beta+2 \gamma e
$$

and therefore

$$
\frac{d}{d e} \frac{d}{d I^{2}}\left(A I^{2}\right)=\beta+2 \gamma e+I^{2}\left(\begin{array}{l}
d \beta \\
d I^{2}
\end{array}+2 e \frac{d \gamma}{d I^{2}}\right) \cdots\left(5^{6}\right) .
$$

The right hand side of this equation changes sign when $e$ passes through the value

$$
-\frac{1}{2}\left(\beta+I^{2} \frac{d \beta}{d I^{2}}\right) /\left(\gamma+I^{2} \frac{d \gamma}{d I^{2}}\right) \cdots \cdots \cdots(57) \text {. }
$$

Now the effects of strain on the intensity of magnetization and of magnetization upon strain depend by (32) and (47) upon the value of 


$$
\frac{d}{d e} \frac{d}{d I^{2}}\left(A I^{2}\right)
$$

so that we should expect the effect of magnetization on the strain of an iron rod to depend upon the strain previously existing in the rod, in such a way that when the strain was less than a critical value magnetization would increase the length of the rod, and when it was greater than this value magnetization would have the contrary effect and tend to shorten the rod. This agrees with the result of Joule's experiments as he found that when the soft iron wires were stretched beyond a certain limit they became shorter instead of longer when they were magnetized.

38. So far we have only considered the effect of expansion and contraction upon the intensity of magnetization and vice-versâ. We can however in a similar way discuss the effects of torsion upon the magnetic properties of iron wire.

Let us now suppose that twist is the only strain in an iron wire which is longitudinally magnetized and has a twist $c$. about its axis, then, using the same notation as before, the terms in the Lagrangian function depending upon strain and magnetization are

$$
\frac{1}{2} A \eta^{2} \xi^{2}+H \eta \xi-\frac{1}{2} n c^{2}
$$

By a similar method to that employed in the case of dilatations we can prove that the twist $c$ due to the same cause as that which makes the intensity of magnetization alter with the torsion is given by the equation

$$
\frac{d c}{d I^{2}}=\frac{\mathrm{I}}{n} \frac{d}{d I^{2}} \frac{d}{d c}\left\{A I^{2}\right\} \ldots \ldots \ldots \ldots . . .\left(5^{8}\right) .
$$

Now when a twisted bar is magnetized it untwists to a certain extent if the magnetization is intense, but the twist increases if the magnetization is weak. If however the bar 
initially has no twist in it then it neither twists nor untwists when it is magnetized (Wiedemann's Elektricität, 111. p. 692).

This shows that if $A$ be expanded in powers of $c$ the first power must be absent, otherwise by equation $\left(5^{8}\right)$ an untwisted bar would twist when it was magnetized. Hence $A$ inust contain a term in $c^{2}$ and therefore the coefficient of $c^{2}$ in the Lagrangian function must contain a term proportional to $I^{2}$. Now the coefficient of $c^{2}$ in the Lagrangian function is proportional to the coefficient of rigidity and hence we see that the rigidity of iron wire will be altered by magnetization.

Since the twist diminishes with strong magnetization we see by equation $(58)$ that the coefficient of $c$ in

$$
\frac{\mathrm{I}}{n} \frac{d}{d c} \frac{d}{d I^{2}}\left(A I^{2}\right)
$$

must be negative when $I$ is large and hence that the coefficient of $c^{2}$ in $A$ must be negative. Let us call this coefficient $-\gamma^{\prime}$, the coefficient of $c^{2}$ in the Lagrangian function is

$$
-\frac{1}{2} \gamma^{\prime} I^{2}-\frac{1}{2} n
$$

but the apparent coefficient of rigidity is twice the coefficient of $-c^{2}$ in the Lagrangian function so that in this case the apparent coefficient of rigidity is

$$
n\left\{\mathrm{I}+\frac{\mathrm{I}}{n} \gamma^{\prime} I^{2}\right\} \text {. }
$$

Thus in this case the effect of strong magnetization is to increase the rigidity, so that the same couple will not twist the wire as much when it is strongly magnetized as when it is unmagnetized.

When the intensity of magnetization is small the opposite will be the case, as in this case the twist in a wire increases when it is longitudinally magnetized.

Since

$$
\frac{\mathbf{r}}{k}=-\frac{d}{d I^{2}}\left\{A I^{2}\right\}
$$


and by (47)

$$
\frac{d I}{d c}\left(\mathrm{I}-H \frac{d k}{d i}\right)=H \frac{d k}{d c}
$$

and also

$$
\frac{d}{d I^{2}} \frac{d}{d c}\left(A I^{2}\right)=\frac{d}{d c} \frac{d}{d I^{2}}\left(A I^{2}\right)+\frac{d^{2}}{d c^{2}}\left(A I^{2}\right) \frac{d c}{d I^{2}} \ldots(59) \text {. }
$$

we have

$$
\frac{d c}{d I^{2}}\left\{\mathrm{I}-\frac{\mathrm{I}}{n} \frac{d^{2}}{d c^{2}}\left(A I^{2}\right)\right\}=\frac{\mathrm{I}}{n k I} \frac{d I}{d c}\left(\mathrm{I}-H \frac{d k}{d c}\right)
$$

or approximately since $d^{2}\left(A I^{2}\right) / n d c^{2}$ is very small

$$
\frac{d c}{d l^{2}}=\frac{\mathrm{I}}{n k I} \frac{d I}{d c}\left(\mathrm{I}-H \frac{d k}{d c}\right) \ldots \ldots \ldots(60) .
$$

We see by this equation that when the magnetization is so strong that magnetizing the wire diminishes the twist in it, then twisting the wire will diminish the intensity of magnetization. On the other hand when the intensity of magnetization is so small that magnetizing the wire increases the twist in it then twisting the wire will increase the intensity of magnetization.

The reciprocal relations between torsion and magnetization have been experimentally investigated by Wiedemann (Lehre z'on der Elektricität, I11. p. 692) and he arranges the corresponding results in parallel columns. These are also quoted in Prof. Chrystal's article on Magnetism in the Encyclopadia Britannica. The following is one set of the corresponding statements.

" 5. If a wire under the influence of a twisting strain is magnetized, the twist increases with weak but diminishes with strong magnetization."

"V. If a bar under the influence of a longitudinal magnetizing force is twisted the magnetization increases with small twists but decreases with large ones."

Comparing these statements with the results we have previously obtained we see that whether the first part of $\mathrm{V}$ is 
true or not depends upon the intensity of magnetization. If the twist be of such a magnitude that 5 is true, then the first part of $\mathrm{V}$ is true if the magnetization is weak, but the opposite is true if the magnetization is strong. Further since by $\mathrm{V}$ the influence of twist on magnetization depends upon the size of the twist, it follows by equation (60) that the influence of magnetization upon twist must depend upon the size of the twist-so that 5 is only true when the twist is on one side of a critical value, when it is on the other side the contrary is true.

The existence of a critical twist as well as a critical magnetization makes the verbal enunciation of the relations between torsion and magnetization cumbrous; they are all however expressed by equation (60).

39. Strains in a dielectric produced by the electric field. The strains produced in a dielectric by the electric field can be found by a method so similar to that used in the last two paragraphs that we shall consider them here though they have no connexion with the terms in the Lagrangian function which we have been considering.

Let $p, q, r$ be the electric displacements parallel to the axes of $x, y, z$ respectively, then if the body is isotropic, the terms in the Lagrangian function of unit volume of the dielectric which depend upon the coordinates fixing the strains and electric configuration if the dielectric is free from torsion are,

$$
\begin{aligned}
& (X p+Y q+Z r)-\frac{2 \pi}{K}\left\{p^{2}+q^{2}+r^{2}\right\} \\
& \quad-\frac{1}{2} m(e+f+g)^{2}-\frac{1}{2} n\left(e^{2}+f^{2}+g^{2}-2 e f-2 e r-2 f_{\delta}\right) \ldots(6 \mathrm{r})
\end{aligned}
$$

where $e, f, g$ are the dilatations parallel to the axes of $x, y, z$ respectively, $K$ the specific inductive capacity of the dielectric and $X, Y, Z$ the electromotive forces parallel to the axes 
of $x, y, z$. Then we see as in $\$ 35$ that $e, f, g$ the strains due to the dependence of $K$ upon the strains in the dielectric are given by the equations

$$
\frac{d L}{d e}=0, \frac{d L}{d f}=0, \quad \frac{d L}{d y}=0,
$$

where $L$ stands for the expression (6I).

Substituting for $L$ its value these equations become respectively

$$
\left.\begin{array}{l}
2 \pi\left\{p^{2}+q^{2}+r^{2}\right\} \frac{d}{d e} \frac{\mathrm{I}}{K}+m(e+f+g)+n(e-f-g)=0 \\
2 \pi\left\{p^{2}+q^{2}+r^{2}\right\} \frac{d}{d f} \frac{\mathrm{I}}{K}+m(e+f+g)+n(f-e-g)=0 \\
2 \pi\left\{p^{2}+q^{2}+r^{2}\right\} \frac{d}{d g} \frac{\mathrm{I}}{K}+m(e+f+g)+n(g-e-f)=0
\end{array}\right\}
$$

Now $\quad p=\frac{\mathrm{I}}{4 \pi} K X, q=\frac{\mathrm{I}}{4 \pi} K Y, \quad r=\frac{\mathrm{I}}{4 \pi} K Z$.

So that if

$$
R^{2}=X^{2}+Y^{2}+Z^{2}
$$

we get from equations (62)

$$
e=\frac{R^{2}}{\mathrm{I} 6 \pi} \frac{\mathrm{I}}{n\left(3^{m-n}\right)}\left\{2 m \frac{d K}{d e}-(m-n)\left(\frac{d K}{d f}+\frac{d K}{d g}\right)\right\} .
$$

with symmetrical expressions for $g$ and $h$.

The expansion in volume

$$
e+f+g
$$

is given by the equation

$$
e+f+g=\frac{R^{2}}{8 \pi} \frac{\mathrm{I}}{3 m-n}\left\{\frac{d K}{d e}+\frac{d K}{d f}+\frac{d K}{d g}\right\} \ldots . .(64) .
$$

Just as in the analogous case of magnetism these are not the only strains produced in the dielectric by the electric field. The term $(X p+Y q+Z r)$ which occurs in the Lagrangian function can be shown to involve the same 
distribution of strain in the dielectric as would be produced by the distribution of stress which Maxwell supposes to exist in the electric field, viz. a tension $K R^{2} / 8 \pi$ along the lines of force and a pressure of the same intensity at right angles to them. The effect of this distribution of stress will be of the same character for all dielectrics, and its nature depends more upon the distribution of force throughout the electric field than upon the nature of the dielectric. The experiments of Quincke (Phil. Mag. x. p. $3 \circ, 1880$ ) and others show that the behaviour of different dielectrics when placed in the same electric field is very different. Thus, for example, though most dielectrics expand when placed in an electric field, the fatty oils on the contrary contract. This difference of behaviour shows that in many cases at any rate, the strains due to the same cause as that which makes the specific inductive capacity depend upon the strain are greater than those produced by Maxwell's distribution of stress.

Quincke has shown that the coefficients of elasticity of a dielectric are altered when an electric displacement is produced in it, this shows that $\mathbf{I} / K$ when expanded in powers of $e$ must contain a term in $e^{2}$ and is another proof that the specific inductive capacity depends upon the strain in the dielectric. Since part of the strain of a dielectric in an electric field is due to the same cause as that which makes the specific inductive capacity depend upon strain, the expression for $\mathrm{x} / K$ when expanded in powers of $e$ must contain the first power of the strains as well as the second, as if it only contained the second powers placing the dielectric in an electric field would merely be equivalent to changing the coefficients of elasticity of the body and so could not strain the body if it were previously free from strain.

No experiments seem to have been made to determine 
directly the values of $d K / d e, d K / d f \& c$., and the experimental difficulties which would have to be overcome in order to do this are much greater than those in the corresponding case in magnetism. The dependence of $K$ upon strain is probably much less than that of $k$, the coefficient of magnetic induction. For the specific inductive capacity seems to be much more independent of the molecular state of the dielectric than the coefficient of magnetic induction is of the molecular state of soft iron. Thus there is a comparatively small difference between the specific inductive capacities of various substances, while the coefficient of magnetic induction of iron is enormously greater than that of any other substance. Again, the coefficient of magnetic induction is known to be much affected by changes in temperature; while some recent experiments made by $\mathrm{Mr}$ Cassie in the Cavendish Laboratory have shown that the effect of changes of temperature on the specific inductive capacities of ebonite, mica and glass is small, amounting in the case of glass, for which it is largest, to I part in 400 for each degree centigrade of temperature. No experiments seem to have been made on the effect of torsion on electrification or of electrification upon torsion.

40. Influence of inertia on magnetic phenomena. In the preceding investigations we have supposed the magnetic changes to take place so slowly that the effects of inertia may be neglected. If however a change in the magnetization involves, as it does according to all molecular theories of magnetism, motion of the molecules of the magnet, then magnetism must behave as if it possessed inertia.

In soft iron and steel the conditions are made so complex by the effects of magnetic friction, magnetic retentiveness and permanent magnetism, that it would be difficult to 
disentangle the effects of inertia proper from other complications. The effect, if it exists, would probably be detected most easily in the case of crystals, as only one of these, quartz, has ever been suspected of showing residual magnetism (see Tumlirz, Wied. Ann. xxvir. p. 133, r 886). The effect of inertia would be to introduce into the equations of magnetization a term

$$
M \frac{d^{2} I}{d t^{2}}
$$

where $I$ is the intensity of magnetization. The equations of magnetization would therefore be of the form

$$
M \frac{d^{2} I}{d t^{2}}+\frac{I}{k}=H \ldots \ldots \ldots \ldots \ldots \ldots \ldots\left(6_{5}\right),
$$

where $H$ is the external magnetic force.

If $H$ is periodic and varies as $e^{i p t}$ then by (65)

$$
I=\frac{k H}{\mathrm{I}-k M \rho^{2}}
$$

so that if $p$ be so large that $k M p^{2}>\mathrm{I}$, the crystal if paramagnetic for a steady magnetic force will be diamagnetic for a variable one and vice versa.

Changes of this kind could be detected very readily if the crystal were freely suspended in the magnetic field, for when $p^{2}$ passed through the value $\mathrm{I} / k M$ the crystal would swing through a right angle.

4I. The term $(\xi y) \xi \dot{y}$ in the Lagrangian function. We have considered the terms depending upon the squares of the velocities of the electrical coordinates, and those depending solely on the magnetic coordinates, let us now consider those terms in the expression for the kinetic energy which involve the product of the velocities of a magnetic and an electrical coordinate.

It is proved in Maxwell's Electricity and Magnetism 
(\$ 634) that when a current whose components are $u, v, w$ flows through the element of volume $d x d y d z$ and the volume $d x^{\prime} d y^{\prime} d z^{\prime}$ is magnetized to the intensities $A, B, C$ parallel to the axes of $x, y, z$ respectively, then the kinetic energy $L$ possessed by the system is

$$
\begin{aligned}
& {\left[u\left\{B \frac{d p}{d z^{\prime}}-C \frac{d p}{d y^{\prime}}\right\}+v\left\{C \frac{d p}{d x^{\prime}}-A \frac{d p}{d z^{\prime}}\right\}\right.} \\
& \left.+w\left\{A \frac{d p}{d y^{\prime}}-B \frac{d p}{d x^{\prime}}\right\}\right] d x d y d z d x^{\prime} d y^{\prime} d z^{\prime} \ldots(67),
\end{aligned}
$$

where $p$ is the reciprocal of the distance between the elements $d x d y d z$ and $d x^{\prime} d y^{\prime} d z^{\prime}$.

Now we represent the intensity of magnetization by $\eta \xi$ where $\xi$ is the momentum corresponding to a kinosthenic or speed coordinate and $\eta$ is a vector quantity.

Since $\eta$ is a vector quantity it may be resolved into components parallel to the axes of $x, y, z$. Let us denote these components by $\lambda, \mu, v$ respectively, then we may put

$$
A=\lambda \xi, \quad B=\mu \xi, \quad C=\nu \xi .
$$

Making this substitution we have

$$
\begin{aligned}
L=[u(\mu & \left.\frac{d p}{d z^{\prime}}-v \frac{d p}{d y^{\prime}}\right)+v\left(v \frac{d p}{d x^{\prime}}-\lambda \frac{d p}{d z^{\prime}}\right) \\
& \left.+w\left(\lambda \frac{d p}{d y^{\prime}}-\mu \frac{d p}{d x^{\prime}}\right)\right] \xi d x d y d z d x^{\prime} d y^{\prime} d z^{\prime} \ldots \text { (68). }
\end{aligned}
$$

So that these terms are of the form

$$
\{\xi y\} \dot{y} \xi \text {. }
$$

Considering the Lagrangian Equation for the electrical coordinate, we see that there is an electromotive force parallel to the axis of $x$ on the element $d x d y d z$ equal to

$$
-\frac{d}{d t} \frac{d L}{d u},
$$


so that, per unit volume, this force equals

or

$$
\begin{aligned}
& -\frac{d}{d t} \xi\left\{\mu \frac{d p}{d z^{\prime}}-\nu \frac{d p}{d y^{\prime}}\right\} d x^{\prime} d y^{\prime} d z^{\prime} \\
& -\frac{d}{d t}\left\{B \frac{d p}{d z^{\prime}}-C \frac{d p}{d y^{\prime}}\right\} d x^{\prime} d y^{\prime} d z^{\prime}
\end{aligned}
$$

with corresponding expressions for the electromotive forces parallel to the axes of $y$ and $z$.

These are the usual expressions for the electromotive forces due to the variations of the magnetic field.

The magnetic force parallel to $x$ acting on the element $d x^{\prime} d y^{\prime} d z^{\prime}$ is by $\S 33$ equal to

$$
\frac{1}{\xi} \frac{d L}{d \lambda}
$$

so that the magnetic force parallel to $x$ per unit volume is equal to

$$
\left(w \frac{d p}{d y^{\prime}}-v \frac{d p}{d z^{\prime}}\right) d x d y d z \ldots \ldots \ldots \ldots . . .(70),
$$

with similar expressions for the magnetic forces parallel to the axes of $y$ and $z$. These expressions agree with those given by Ampère for the magnetic force produced by a system of currents.

Again there is a mechanical force acting on the element $d x d y d z$ whose component parallel to the axis of $x$ is

If we call

$$
\frac{d L}{d x} .
$$

$\left.\begin{array}{l}\left(\mu \frac{d p}{d z^{\prime}}-\nu \frac{d p}{d y^{\prime}}\right) \xi d x^{\prime} d y^{\prime} d z^{\prime} \text { or }\left(B \frac{d p}{d z^{\prime}}-C \frac{d p}{d y^{\prime}}\right) d x^{\prime} d y^{\prime} d z^{\prime} \\ \left(\nu \frac{d p}{d x^{\prime}}-\lambda \frac{d p}{d z^{\prime}}\right) \xi d x^{\prime} d y^{\prime} d z^{\prime} \text { or }\left(C \frac{d p}{d x^{\prime}}-A \frac{d p}{d z^{\prime}}\right) d x^{\prime} d y^{\prime} d z^{\prime} \\ \left(\lambda \frac{d p}{d y^{\prime}}-\mu \frac{d p}{d x^{\prime}}\right) \xi d x^{\prime} d y^{\prime} d z^{\prime} \text { or }\left(A \frac{d p}{d y^{\prime}}-B \frac{d p}{d x^{\prime}}\right) d x^{\prime} d y^{\prime} d z^{\prime}\end{array}\right\}$

$F, G, H$ respectively, then $F, G, H$ are the same as the 
MECHANICAL FORCE DUE TO A CURRENT. 69 quantities denoted by the same symbols in Maxwell's Electricity and Magnetism.

Since the force on the element $d x d y d z$ is

$$
\frac{d L}{d x}
$$

we see that the force on unit volume may be written

or

$$
u \frac{d F}{d x}+v \frac{d G}{d x}+w \frac{d H}{d x},
$$

$v\left\{\frac{d G}{d x}-\frac{d F}{d y}\right\}-w\left\{\frac{d F}{d z}-\frac{d H}{d x}\right\}+u \frac{d F}{d x}+v \frac{d F}{d y}+w \frac{d F}{d z} \ldots(72)$.

This differs from Maxwell's expression for the same force by the term

$$
u \frac{d F}{d x}+v \frac{d F}{d y}+w \frac{d F}{d z} .
$$

Since

$$
\frac{d u}{d x}+\frac{d v}{d y}+\frac{d w}{d z}=0
$$

it follows that

$$
\iiint\left(u \frac{d F}{d x}+v \frac{d F}{d y}+w \frac{d F}{d z}\right) d x d y d z=0
$$

if all the circuits are closed. So that as long as the circuits are closed the effect of the translatory forces is the same as if they were given by Maxwell's expressions.

In the above investigation we have assumed that we could move the element without altering the current; if we suppose the current to move with the elements we shall get Maxwell's expression exactly.

The components parallel to $y$ and $z$ of the force on the element $d x d y d z$ are given by expressions corresponding to (72).

The force parallel to $x$ on the magnetized volume $d x^{\prime} d y^{\prime} d z^{\prime}$, is

$$
\frac{d L}{d x^{\prime}}
$$


so that the force parallel to $x$ per unit volume is

$$
\begin{array}{r}
\left\{(v C-w B) \frac{d^{2} p}{d x^{\prime 2}}+(w A-u C) \frac{d^{2} p}{d x^{\prime} d y^{\prime}}+(u B-v A) \frac{d^{2} p}{d x^{\prime} d z^{\prime}}\right\} \\
d x d y d z \ldots(73)
\end{array}
$$

with corresponding expressions for the forces parallel to $y$ and $z$.

Thus the force on the magnet is equal and opposite to that on the current.

We see by this example how from the existence of a single term in the expression for $L$ we can deduce the laws of the induction of currents, the production of a magnetic field by a current, the mechanical force on a current in a magnetic field and the mechanical force on a magnet placed near a current.

42. Twist in a magnetized iron wire produced by a current. Prof. G. Wiedemann (Elektricität, III. p. 689) has shown that when a current flows along a longitudinally magnetized wire, it produces a couple tending to twist the wire. This shows that there must be a term in the Lagrangian function for the wire of the form

$$
f(c) \dot{y} \eta \xi \ldots \ldots \ldots \ldots \ldots \ldots(74),
$$

where $\dot{y}$ is the current flowing along the wire, $\eta \xi$ the intensity of magnetization $I$, and $c$ the twist about the axis of the wire, $f(c)$ being some function of $c$. Applying Hamilton's principle to this term we see that it indicates the existence of a couple tending to twist the wire equal to

or

$$
j \eta \xi \frac{d f(c)}{d c}
$$

$$
\dot{y} I \frac{d f(c)}{d c}
$$

Applying Lagrange's equation for the $y$ coordinate to this term we see that since the electromotive force tending to 
increase $y$ is

$$
\ldots . . .-\frac{d}{d t} \frac{d L}{d \dot{y}},
$$

the existence of this term shows that there is an electromotive furce along the wire equal to

$$
-\frac{d}{d t}\{f(c) \eta \xi\}
$$

that is

$$
-\frac{d}{d t}\{f(c) I\}
$$

Thus twisting a longitudinally magnetized iron wire must produce an electromotive force which lasts as long as the twist is changing, and any alteration in the longitudinal magnetization of a twisted iron wire must produce one lasting as long as the magnetization changes. Hence Faraday's rule that the electromotive force round the circuit due to induction equals the rate of diminution in the number of lines of force passing through it, will not apply to the case of a twisted iron wire, for we might get an electromotive force round a circuit made of such a wire by moving it in the plane of the magnetic force, and in this case there is no alteration in the number of lines of force passing through the circuit.

The production of an electromotive force by twisting a longitudinally magnetized iron wire has been experimentally verified.

Again, if we consider Lagrange's equations for the coordinates fixing the magnetic configuration, since any term in the Lagrangian function indicates an effect similar to that which would be produced by an external magnetic force equal to

$$
\frac{1}{\xi} \frac{d L}{d \eta}
$$

we see that the term we are considering indicates the 
existence of a magnetizing force on the wire equal to

$$
f(c) \dot{y} \ldots \ldots \ldots \ldots \ldots \ldots \ldots(77)
$$

tending to magnetize it longitudinally. So that if a current of electricity passes along a twisted wire or if a wire conveying a current of electricity be twisted the wire will be longitudinally magnetized. These effects have been observed by Prof. G. Wiedemann (Elektricität, III. p. 692).

43. Hall's phenomenon. The terms we are considering, involving both the electric and magnetic coordinates, are also interesting from their connexion with Hall's phenomenon, for as we shall see directly this phenomenon indicates the existence in the Lagrangian function of terms of this kind. Hall discovered (Phil. Mag. x. 30r,) that when currents are flowing through a conductor placed in a magnetic field, there is an electromotive force due to the field even though it remains constant, and that this electromotive force at any point is parallel and proportional to the mechanical force acting on the conductor conveying the current at that point. Thus the electromotive force is at right angles both to the direction of the current and the magnetic induction, and its components parallel to the axes of $x, y, z$ are respectively given by the expressions

$$
\begin{aligned}
& -C^{\prime}(\gamma \dot{g}-\beta \dot{h}), \\
& -C^{\prime}(\alpha \dot{h}-\gamma \dot{f}), \\
& -C^{\prime}(\beta \dot{f}-\alpha \dot{g}),
\end{aligned}
$$

where $C^{\prime}$ is a constant depending upon the nature of the medium through which the current is flowing, $\alpha, \beta, \gamma$ are the components of the magnetic force and $f, g, h$ are the components of the electric displacement if the medium is a dielectric, if the medium is a conductor $\dot{f}, \dot{g}, \dot{h}$ are the components of the electric current. 
Prof. Fitzgerald ("On the Electromagnetic Theory of the Reflection and Refraction of Light," Phil. Trans. I880, Part II.) and Mr Glazebrook (Phil. Mag. Xı. p. 397, I88I) have shown that the existence of this force proves that there is a term equal to

$$
\frac{1}{2} C^{\prime}\{\dot{f}(\gamma g-\beta h)+\dot{g}(\alpha h-\gamma f)+\dot{h}(\beta f-a g)\} \ldots(78)
$$

in the expression for the Lagrangian function of unit volume of the medium.

Let us consider the Lagrangian equation for the electric displacement $f$. It indicates the existence of an electromotive force parallel to the axis of $x$ equal to

or in this case

$$
-\frac{d}{d t} \frac{d L}{d \dot{f}}+\frac{d L}{d f}
$$

$$
C^{\prime}(\beta \dot{h}-\gamma \dot{g})+\frac{1}{2} C^{\prime}(\dot{\gamma} g-\dot{\beta} h) \ldots \ldots \ldots(79) .
$$

The first of these terms corresponds to the Hall effect, the second to an electromotive force tending to displace the lines of electrostatic force.

This latter force is at right angles both to the direction of electric displacement and to that in which the change in the magnetic force is greatest; the magnitude of the force is

$$
\frac{1}{2} C^{\prime} \dot{H} D \sin \theta,
$$

where $D$ is the resultant electrostatic displacement, $\dot{H}$ the rate of change of the magnetic force and $\theta$ the angle between the electric displacement and the direction in which the change in the magnetic force is greatest.

If $P$ be the original electromotive force then since the Hall electromotive force is very small we have approximately

$$
D=\frac{K}{4 \pi} P,
$$

where $K$ is the specific inductive capacity of the medium. 
Thus the ratio of the disturbing force we are considering to the original electromotive force is

$$
\frac{\mathrm{I}}{8 \pi} C^{\prime} K \dot{H} \sin \theta \text {. }
$$

Now Hall's experiments show that $C^{\prime}$ in electromagnetic measure is at most of order $1 \mathrm{O}^{-5}$; and $K$ is of the order $1 \mathrm{O}^{-21}$ so that the ratio of the disturbing force to the original force is of the order

$$
{ }_{10}^{-27} \dot{H} \sin \theta \text {, }
$$

and is thus much too small for there to be any chance of its detection by experiment.

We see too from the expression for this force that it absolutely vanishes when both the electric displacement and the magnetic force are stationary, and these were the conditions when Hall tried unsuccessfully to detect the existence of his effect in an insulator (Phil. Mag. x. p. 304, 1880.)

Let us now consider Hall's effect from the point of view of magnetic instead of electromotive force. Perhaps the easiest way to do this will be to suppose that the magnetic forces are produced by an element of volume $d x^{\prime} d y^{\prime} d z^{\prime}$ magnetized to intensities $A, B, C$ parallel to the axes of $x, y, z$ respectively. If $\Omega$ is the magnetic potential of this element at a distance $r$, then, for a point outside the magnet

$$
\mathbf{\Omega}=-\left(A \frac{d}{d x} \frac{\mathrm{I}}{r}+B \frac{d}{d y} \frac{\mathrm{I}}{r}+C \frac{d}{d z} \frac{\mathrm{r}}{r}\right) d x^{\prime} d y^{\prime} d z^{\prime} \ldots(80),
$$

and

$$
\alpha=-\frac{d \Omega}{d x}, \beta=-\frac{d \Omega}{d y}, \quad \gamma=-\frac{d \Omega}{d z} .
$$

Substituting these values for $\alpha, \beta, \gamma$ in the expression (78) we see that there is a term in the Lagrangian function equal to 


$$
\begin{aligned}
& \frac{\mathrm{I}}{2} C^{\prime}\left[A\left\{\{\dot{g} h-g \dot{h}\} \frac{d^{2}}{d x^{2}} \frac{\mathrm{I}}{r}+\{\dot{h} f-h \dot{h}\} \frac{d^{2}}{d x d y} \frac{\mathrm{I}}{r}+\{\dot{f} g-f \dot{g}\} \frac{d^{2}}{d x d z} \frac{\mathrm{I}}{r}\right\}\right. \\
& +B\left\{\{\dot{g} h-g \dot{h}\} \frac{d^{2}}{d x d y} \frac{\mathrm{I}}{r}+\{\dot{h} f-h \dot{f}\} \frac{d^{2}}{d y^{2}} \frac{\mathrm{I}}{r}+\{\dot{f g}-f \dot{g}\} \frac{d^{2}}{d y d z} \frac{\mathrm{I}}{r}\right\} \\
& \left.+C\left\{\{\dot{g} h-g \dot{h}\} \frac{d^{2}}{d x d z} \frac{\mathrm{I}}{r}+\{\dot{h} f-h \dot{f}\} \frac{d^{2}}{d y d z} \frac{\mathrm{I}}{r}+\{\dot{f g}-f \dot{g}\} \frac{d^{2}}{d z^{2}} \frac{\mathrm{I}}{r}\right\}\right] \\
& \quad \text { If as on page }(67) \text { we put } \quad d x^{\prime} d y^{\prime} d z^{\prime} .
\end{aligned}
$$

$$
A=\xi \lambda ; B=\xi \mu ; C=\xi \nu
$$

the magnetic force on the element $d x^{\prime} d y^{\prime} d z^{\prime}$ parallel to the axis of $x$ will be

$$
\frac{\mathbf{r}}{\xi} \frac{d L}{d \lambda},
$$

so that in this case the magnetic force parallel to $x$ per unit volume is

$\frac{\mathbf{I}}{2} C^{\prime}\left[\{\dot{g} h-g \dot{h}\} \frac{d^{2}}{d x^{2}} \frac{\mathbf{I}}{r}+\{\dot{h} f-h \dot{h}\} \frac{d^{2}}{d x d y} \frac{\mathbf{I}}{r}+\{\dot{f g}-f \dot{g}\} \frac{d^{2}}{d x d z} \frac{\mathbf{I}}{r}\right]$, or if

$$
\psi=\frac{\mathrm{I}}{2} C^{\prime}\left[\{\dot{g} h-g \dot{h}\} \frac{d}{d x}, \frac{\mathrm{I}}{r}+\{\dot{h} f-h \dot{f}\} \frac{d}{d y}, \frac{\mathrm{I}}{r}+\{\dot{f g}-f \dot{\delta}\} \frac{d}{d z^{\prime}} \frac{\mathrm{I}}{r}\right]
$$

then the magnetic force at the points $x^{\prime}, y^{\prime}, z^{\prime}$ parallel to the axis of $x$ due to the electric displacements $f, g, h$ through unit volume at the point $(x y z)$ is

$$
\frac{d \psi}{d x}, \ldots \ldots \ldots \ldots \ldots \ldots . . .
$$

similarly the magnetic forces parallel to $y$ and $z$ are

$$
\frac{d \psi}{d y^{\prime}}
$$

and

$$
\frac{d \psi}{d z^{\prime}}
$$

respectively. 
If we have electric displacements distributed throughout a volume of any size, then the components of the magnetic force parallel to the axes of $x, y, z$ due to the same cause as that which produces Hall's effect are

respectively, where

$$
\frac{d \Psi}{d x^{\prime}}, \frac{d \Psi}{d y^{\prime}}, \frac{d \Psi}{d z^{\prime}}
$$

$$
\Psi=\iiint \psi d x d y d z \ldots \ldots \ldots \ldots \ldots . . .(85),
$$

the integration being extended over the volume throughout which there are electric displacements.

If the point at which we wish to find the magnetic force is inside the volume occupied by the electric displacements we must modify the preceding results. Let us suppose that we have a small sphere whose centre is at the point where we require the magnetic force, magnetized to the intensities $A, B, C$ parallel to the axes of $x, y, z$ respectively. Then inside the sphere

$$
\alpha=\frac{4 \pi}{3} A, \beta=\frac{4 \pi}{3} B, \gamma=\frac{4 \pi}{3} C .
$$

So that the Lagrangian function for an element of volume $d x d y d z$ inside the sphere is

$$
\frac{\mathrm{I}}{2} C^{\prime} \frac{4 \pi}{3}\{A(\dot{g} h-g \dot{h})+B(\dot{h} f-h \dot{f})+C(\dot{f g}-f \dot{g})\} d x d y d z
$$

hence the components of the magnetic force due to the electric displacement at the point where the force is measured are

$$
\begin{aligned}
& \frac{2}{3} \pi C^{\prime}(\dot{g} h-g \dot{h}), \\
& \frac{2}{3} \pi C^{\prime}(\dot{h} f-h \dot{h}), \\
& \frac{2}{3} \pi C^{\prime}(\dot{f} g-f \dot{g}) .
\end{aligned}
$$

So that the general expression for the components of the magnetic force due to the cause producing the Hall effect are 


$$
\left.\begin{array}{c}
\frac{d \Psi}{d x^{\prime}}+\frac{2}{3} \pi C^{\prime}(\dot{g} h-g \dot{h}) \\
\frac{d \Psi}{d y^{\prime}}+\frac{2}{3} \pi C^{\prime}(\dot{h} f-h \dot{f}) \\
\frac{d \Psi}{d z^{\prime}}+\frac{2}{3} \pi C^{\prime}(\dot{f g}-f \dot{g})
\end{array}\right\}
$$

where $f, g, h$ are the components of the electric displacement at the point where the magnetic force is measured and $\Psi$ is given by equation (8I). Since $C^{\prime}$ is a very small quantity, as are also $f, g, h$, these forces will be very small, and it is only when $f, g, h$ vary very rapidly that we could expect to have any chance of detecting them. We shall therefore calculate the magnitude of these forces when the electric displacement changes with the greatest rapidity we can produce in an experiment. This if the Electromagnetic Theory of Light is true will be when the electric displacements are those which accompany the propagation of light.

Let us suppose that we have a circularly polarized ray travelling along the axis of $z$ and that the electric displacements are given by the equations

$$
\left.\begin{array}{l}
f=w \cos \frac{2 \pi}{\lambda}(v t-z) \\
g=w \sin \frac{2 \pi}{\lambda}(v t-z) \\
h=0
\end{array}\right\} \ldots \ldots \ldots \ldots(87)
$$

where $w$ is the amplitude of the oscillation, $\lambda$ the wave length and $v$ the velocity of propagation of light.

Substituting these values we see

$$
\left.\begin{array}{l}
\dot{g} h-g \dot{h}=0 \\
\dot{h} f-h \dot{f}=0 \\
\dot{f g}-f \dot{g}=-w^{2} \frac{2 \pi v}{\lambda}
\end{array}\right\}
$$


If we consider a long cylindrical beam of light

$$
\Psi=0
$$

and thus by equation (86) the circularly polarized ray produces a magnetic force in the direction along which it is propagated equal to

$$
-\frac{2}{3} \pi C^{\prime} z \epsilon^{2} \cdot \frac{2 \pi z^{\prime}}{\lambda},
$$

we can deduce the value of $w$ for strong sunlight from the data given in Maxwell's Electricity and Magnetism, Vol. II. $\S 793$. The maximum electromotive force in this case is given as

$$
6 \times 10^{7}
$$

in electromagnetic measure, the maximum value $w$, of the displacement corresponding to this is

or

$$
\frac{K}{4 \pi} 6 \times 10^{2}
$$

$$
\frac{3 \times 10^{7}}{2 \pi v^{2}}
$$

Assuming the wave length to be $6 \times 1 \mathrm{10}^{-5}$, which is a little greater than that of the $D$ line and $C^{\prime}$ to be $10^{-5}$, we see that the magnetic force produced by circularly polarized light as intense as strong sunlight cannot be greater than

$$
2 \times 10^{-18} \text {, }
$$

which is much too small to be detected by experiments.

The direction of the magnetic force is related to the direction of rotation of the electric displacement in a circularly polarized ray like translation and rotation in a left-handed screw.

Prof. Rowland has shown (Phil. Mag. Apr. 188I) that the Hall effect if it existed in transparent bodies (which with the 
exception of electrolytes are all insulators) would account for the rotation of the plane of polarization of light passing through such bodies placed in a magnetic field in which the lines of magnetic force are more or less parallel to the direction of propagation of the light. In this case by the aid of an external magnetic force we rotate the plane of polarization; in the case we have just investigated, which may be looked upon as the converse of this, a beam of circularly polarized light produces a magnetic force parallel to the direction in which it is travelling. 


\section{CHAPTER V.}

RECIPROCAL RELATIONS BETWEEN PHYSICAL FORCES WHEN THE SYSTEMS EXERTING THEM ARE IN A STEADY STATE.

44. THE preceding methods are applicable to systems in all states, whether steady or variable. When however the system is in a steady state the reciprocal relations between the various physical forces become so simple that they seem deserving of special treatment, and we shall accordingly consider them separately.

Let us consider the mutual effect of two quantities fixed by the coordinates $p$ and $q$ upon each other. Let us suppose that we have a force $P$ of type $p$ acting upon the system, then $P$ will alter the coordinate $p$ in a definite way and the amount of the alteration may depend upon the value of the other coordinate $q$. Let us suppose that $q$ suffers a small alteration $\delta q$ and that $\delta P$ is the amount by which $P$ must be increased in order to keep $p$ the same as before. Then since the system is in a steady state if $L$ be the Lagrangian function we have

$$
P=-\frac{d L}{d p}
$$

and

$$
P+\delta P=-\frac{d L}{d p}-\frac{d^{2} L}{d q d p} \delta q
$$


so that

$$
\delta P=-\frac{d^{2} L}{d p d q} \delta q
$$

Now if we have a force $Q$ of type $q$ producing a definite change in the coordinate $q$ then if we alter $p$ by $\delta p$ we must in order to keep $q$ constant alter $Q$ by some quantity $\delta Q$, and sinci

$$
Q=-\frac{d L}{d q}
$$

and

$$
Q+\delta Q=-\frac{d L}{d q}-\frac{d^{2} L}{d p d q} \delta p
$$

we have

$$
\delta Q=-\frac{d^{2} L}{d p d q} \delta p \ldots \ldots \ldots \ldots \ldots . . .(90)
$$

so that by $(89)$ and $(90)$ we have

$$
\left(\frac{d P}{d q}\right)_{p \text { constant }}=\left(\frac{d Q}{d p}\right)_{q \text { constant }} \ldots . . . \cdots . . .(9 \mathrm{I}) .
$$

Or the alteration in $P$ when $q$ is increased by unity, $p$ being constant, is the same as the alteration in $Q$ when $p$ is increased by unity, $q$ being constant. Thus if $P$ depends upon $q$ then $Q$ will depend upon $p$ and vice versâ. And we notice that if by increasing $q$ we increase the "spring" of $p$ then by increasing $p$ we shall increase the "spring" of $q$.

Equation (9I) is analogous to the "thermodynamical relations" given in Maxwell's Theory of Heat, p. $x_{9}$ and forms one of those reciprocal relations which exist in physics and which so often enable us to duplicate discoveries in Physical Science. The consequences of reciprocal relations of a different kind are considered by Lord Rayleigh in the Theory of Sound, Vol. I. Chapter 5 .

As an example of the application of this equation we 
may take the case of a wire bent into any shape by the action of any number of forces two of which are $P$ and $Q$, then the increase in $Q$ required to keep its point of application at rest when $p$ is increased by unity, will also be the amount by which $P$ must be increased to prevent its point of application moving when $q$ is increased by unity.

Or again, we see by this equation that if the force required to produce a given extension in an iron wire is altered by magnetizing the wire then the magnetic force required to magnetize the wire to a given intensity will be altered by straining the wire: and that these alterations will be connected by the following relation, $P$ being the tension, $e$ the extension of the wire, $H$ the magnetic force and $I$ the inţensity of magnetization,

$$
\left(\frac{d P}{d I}\right)_{e \text { constant }}=\left(\frac{d H}{d e}\right)_{I \text { constant }}
$$

Again when a current passes through an electrolyte in solution it decomposes it and the strength of the solution changes, this change in the strength of the solution may, and in general will, change the coefficient of compressibility, the volume and the surface tension of the solution, and in this case equation (9I) shows that the electromotive force required to send a given current through a cell containing the solution will be altered by pressure and by any change in the free surface of the solution. Let $E$ be the electromotive force, $\gamma$ the current, $v$ the volume of the solution, $S$ its surface, and $T$ its surface tension, then in this case for the effect of pressure $p$ we have

$$
\left(\frac{d E}{d v}\right)_{\gamma \text { constant }}=-\left(\frac{d p}{d \gamma}\right)_{v \text { constant }} \ldots \ldots \ldots . . .(93) .
$$

The negative sign is taken because $p$ tends to diminish $v$. 
EFFECT OF PRESSURE ON ELECTROMOTIVE FORCE. 83

If $k$ be the modulus of compression, $v_{0}$ the volume of the solution when free from pressure, then

$$
\begin{gathered}
p=k\left(\mathrm{r}-\frac{v}{v_{0}}\right) \\
\left(\frac{d p}{d \gamma}\right)_{v}=\left(\mathrm{r}-\frac{v}{v_{0}}\right) \frac{d k}{d \gamma},
\end{gathered}
$$

when $\gamma$ is constant, $k$ is also constant so that

and therefore from (93)

$$
\delta p=-\frac{k \delta v}{v_{0}}
$$

$$
\begin{aligned}
d E & =v_{0}\left(\mathrm{I}-\frac{v}{v_{0}}\right) \frac{d k}{d \gamma} \frac{d p}{k} \\
& =v_{0} \frac{\mathrm{I}}{k^{2}} \frac{d k}{d \gamma} p d p
\end{aligned}
$$

so that if the pressure is increased from $P_{1}$ to $P_{2}$ the increase $\delta E$ in the electromotive force required to keep the current constant is given by

$$
\delta E=\frac{1}{2} v_{0}\left\{P_{2}^{2}-P_{1}^{2}\right\} \frac{\mathbf{r}}{k^{2}} \frac{d k}{d \gamma} \ldots \ldots \ldots . .(94) .
$$

To get an idea of the magnitude of this effect let us take the case of a solution of chloride of lithium, the volume of the solution being I cubic centimetre.

The data for calculating $d k / d \gamma$ in this case are the following:

The passage of unit quantity of electricity corresponds to the decomposition of about $4.3 \times 10^{-3}$ grammes of lithium chloride, we shall suppose that none of this is redissolved, then the passage of a unit quantity of electricity will withdraw this quantity of salt from the solution.

Röntgen's and Schneider's experiments (Wiedemann's Annalen, xxix. p. I86, I886) show that the addition of 6 grammes of lithium chloride to 100 cubic centimetres of 
water increases the modulus of compression by about 15 parts in 100 , so that if the increase in the modulus is proportional to the quantity of salt, then the subtraction of $4.3 \times 10^{-3}$ grammes from I cubic centimetre will diminish the modulus by about $\mathbf{r}$ part in 100 , hence

$$
\frac{1}{k} \frac{d k}{d \gamma}=-10^{-8}, \text { approximately. }
$$

Now $k$ for water is about $2.2 \times 10^{10}$, so that if $\delta E$ is the change produced by a pressure of 1000 atmospheres, which in absolute measure is about $10^{\circ}$, we have

$$
\delta E=-\frac{1}{2} \frac{10^{18}}{2 \cdot 2 \times 10^{10}} \frac{1}{10^{2}}=-\frac{1}{4} \times 10^{6},
$$

that is the pressure of 1000 atmospheres would diminish the counter electromotive force by about $\mathbf{r} / 400$ of a volt.

The numbers given by Röntgen and Schneider for the effect of carbonate of soda on the coefficient of compressibility, show that the effect of pressure on a solution of this salt would be much greater than that on the lithium chloride solution.

Let us now suppose that the volume of the solution is altered by the passage of an electric current, but that the coefficient of compressibility is unaltered.

Then since

$$
p=k\left(\mathrm{x}-\frac{v}{v_{0}}\right)
$$

if the passage of the unit of electricity increases the volume by $d v / d \gamma$ we must apply an additional pressure $k d v / v_{0} d \gamma$ to keep the volume constant, so that

$$
\left(\frac{d p}{d \gamma}\right)_{v \text { constant }}=\frac{k}{v_{0}} \frac{d v}{d \gamma}
$$

and the equation ( $9 \mathrm{r})$ becomes 
EFFECT OF PRESSURE ON ELECTROMOTIVE FORCE. 85

$$
\left(\frac{d E}{d v}\right)_{\gamma \text { constant }}=-\frac{k}{v_{0}} \frac{d v}{d \gamma},
$$

so that since $k d v / v_{0}=-d p$, we see from this equation that the change $\delta E$ in the counter electromotive force is given by the equation

$$
\delta \dot{E}=\delta p \frac{d v}{d \gamma} \ldots \ldots \ldots \ldots \ldots \ldots \ldots(95) .
$$

When the electric current goes through a salt solution the changes which take place and which alter the volume are so numerous that it is not possible to calculate from existing data the change which takes place in the volume when unit quantity of electricity passes through the solution. In order to see of what order this effect is likely to be, let us suppose that the change in the volume is comparable with the volume of the salt electrolysed. When unit quantity of electricity goes through a solution of sulphate of potassium it electrolyses about $9 \times 10^{-3}$ grammes of salt, and since the specific gravity of the salt is $2 \cdot 6$, the volume of this is about $3.5 \times 10^{-3}$, hence in this case we may suppose that $d v / d \gamma$ is comparable with $3.5 \times 10^{-3}$ and that the change in the counter electromotive force produced by 1000 atmospheres is of the order

$$
3.5 \times 10^{6},
$$

or about $\mathrm{I} / 28$ of a volt.

We will now consider the case when gas is given off. Let us suppose we are electrolysing water, above which we have air, enclosed by a cylinder with a moveable piston.

If unit quantity of electricity goes through the water, $9 \times 10^{-4}$ grammes of water are electrolysed, the volume of the water therefore diminishes by $9 \times 10^{-4}$ cubic centimetres. At one terminal $10^{-4}$ grammes of hydrogen will be liberated, and $8 \times 10^{-4}$ grammes of oxygen at the uther. 
Let us proceed to find the change in the pressure, the volume remaining constant when unit of electricity passes.

The diminution in pressure due to the disappearance of the water is, if $v$ be the volume of the gas above the water

$$
9 \times 10^{-4} \frac{p}{v},
$$

the increase in pressure due to the $10^{-4}$ grammes of hydrogen is if the temperature is $0^{\circ} \mathrm{C}$.

$$
I \cdot I \times 10^{10} \times \frac{10^{-4}}{v}
$$

and the increase due to the oxygen is one half of this, hence

$$
\left(\frac{d p}{d \gamma}\right)_{v}=\frac{\mathbf{I}}{v}\left\{1 \cdot 65 \times 10^{6}-9 \times 10^{-4} p\right\}
$$

so that by (95)

$$
\frac{d E}{d v}=-\frac{1}{v}\left\{1.65 \times 10^{6}-9 \times p \times 10^{-4}\right\} .
$$

But

so that

$$
\frac{\delta v}{v}=-\frac{\delta p}{p}
$$

$$
\frac{d E}{d p}=\frac{1.65 \times 10^{6}}{p}-9 \times 10^{-4} .
$$

If the pressure is increased from $P_{1}$ to $P_{2}$ the change $\delta E$ in $E$ is given by the equation

$$
\delta E=1^{\circ} 65 \times 10^{6} \times \log \frac{P_{2}}{P_{1}}-9 \times 10^{-4} \times\left(P_{2}-P_{1}\right) .
$$

For a thousand atmospheres the counter electromotive force is increased by

$$
\begin{gathered}
\mathrm{I} \cdot 65 \times 10^{6} \times 6.9-9 \times 10^{-4} \times 10^{9} \text { approximately } \\
=\mathrm{I} \cdot 2 \times 10^{2}-9 \times 10^{5}
\end{gathered}
$$


EFFECT OF SURFACE TENSION ON E. M. F. 87 so that the counter electromotive force is increased by about one-eighth of a volt.

The effect of surface tension is given by

$$
\left(\frac{d E}{d S}\right)_{\gamma \text { constant }}=\left(\frac{d T}{d \gamma}\right)_{S \text { constant }} \ldots \ldots \ldots(96) .
$$

This effect will in general be very small, for example in the case of chloride of lithium, the experiments of Röntgen and Schneider (Wiedemann's Annalen, xxix. p. 209, I 886), show that the addition of 6 parts by weight of lithium chloride to roo of water increases the surface tension by about 3 parts in Ioo. The passage of $\mathbf{I}$ unit of electricity decomposes about $4.3 \times 10^{-3}$ grammes of lithium chloride, so that if $v$ be the volume of the solution

$$
\begin{aligned}
\frac{\mathbf{I}}{T} \frac{d T}{d \gamma} & =-\frac{\mathbf{I}}{v} \times \frac{3}{10^{2}} \times \frac{10^{2}}{6} \times 43 \times 10^{-3} \\
& =-2 \times 10^{-3} \frac{\mathbf{I}}{v} \text { approximately, }
\end{aligned}
$$

and for water

$$
T=8 \mathrm{I}
$$

so that

$$
\frac{d T}{d \gamma}=-\frac{\mathrm{1} 6 \cdot 2 \times 10^{-2}}{2}
$$

and therefore by $(96)$

$$
\frac{d E}{d S}=-\frac{16.2 \times 10^{-2}}{v},
$$

or if the volume remains constant the effect of increasing the surface by $S$ is to diminish the counter electromotive force by

$$
\frac{16.2 \times 10^{-2} S}{v} .
$$

Suppose that the liquid is squeezed out into a thin film 
whose thickness is $t$ then

and

$$
\begin{gathered}
v=S t \\
\delta E=-\frac{16.2 \times 10^{-2}}{t} .
\end{gathered}
$$

If $t$ were of the order of molecular distances say $10^{-7}$ then

$$
\delta E=-16.2 \times 10^{5},
$$

or the counter electromotive force is diminished by about

$$
=\cdot 0 \mathrm{r} 6 \text { volts. }
$$

The preceding investigation is on the supposition that the electrolyte is in contact with the air; if it were in contact with a solid such as glass the withdrawal of the electrolyte from the solution on the passage of the current would increase the surface tension between the liquid and the solid, so that the electromotive force required to decompose an electrolyte in a porous plate would be larger than that required to decompose it when it is in bulk.

Again, the surface tension of liquids is altered when they absorb gases, so that the electromotive force required to decompose an electrolyte which absorbs a gas produced by the passage of the current will be different when the electrolyte fills the interstices of a porous plate from that required when it is in an ordinary electrolytic cell. 


\section{CHAPTER VI.}

EFFECT OF TEMPERATURE UPON THE PROPERTIES OF BODIES.

45. WE have only considered so far the relations between the phenomena in electricity, magnetism and elasticity and have not discussed any phenomenon in which temperature effects occur. We shall now go on however to endeavour to extend the methods we have hitherto used to those cases in which we have to consider the effects of temperature upon the properties of bodies.

Before doing this however we must endeavour to arrive at some dynamical interpretation of temperature. The only case in which a dynamical conception of temperature has been attained is in the Kinetic Theory of Gases, and there the temperature is the mean energy due to the translatory motion of the molecules of the gas. So that if $N$ be the number of molecules of the gas in unit volume $N \theta$ is the energy of translatory motion of the molecules at the temperature $\theta$.

There seems good reason for believing that $N \theta$ is a part of the kinetic energy of the molecules when these are aggregated so as to form a solid or liquid as well as when they form a gas. 
The experiments and ideas which led to the establishment of the principle of the Conservation of Energy at the same time led to the conclusion that the energy of sensible heat is energy due to the motion of the molecules and is therefore part of the kinetic energy of the system. The reader should refer on this point to Maxwell's Theory of Heat, p. 3or. Another reason for supposing that the temperature in the liquid as well as in the gaseous condition is measured by the mean energy of translation of the molecules is, that Van der Wäals (Die Continuität des gasförmigen und flïsigen Zustandes) has given a theory of the molecular constitution of bodies in those states which are intermediate between the liquid and gaseous, in which this supposition is made, and that this theory agrees well with the facts in many important respects. And again since most solids and liquids are capable of getting into a state where their specific heat is constant, that is, where the rise in temperature is proportional to the energy communicated to the system, we are led to suppose that the kinetic energy of some particular kind is a linear function of the temperature.

This following illustration will show that it is probable that when we have two bodies in contact the collisions. between the molecules will tend to equalize the mean energy of this translatory motion when these bodies are solids and liquids as well as when they are gases. The mean translatory energies of two substances in contact thus tend to become equal, so that in this important respect the mean translatory energy has the same property as temperature.

Let us suppose that we have two different substances. composed of molecules $A$ and $B$ respectively, and that the molecules of the two substances are separated by a material. 
plane surface. Let us also suppose that the mass of this plane is large compared with that of a molecule of either substance and that it is prevented by perfectly elastic stops from moving through more than a distance comparable with molecular distances. Since the mass of the plane is very much greater than that of a molecule and since it can only move through a small distance in one direction-the velocity of the plane will be very small compared with that of the molecules-we shall suppose that it is so small that the number of molecules which are moving more slowly than the plane may be neglected, or what amounts to the same thing that all the molecules on the surface of the substances which are moving towards the plane strike it, and that none of those which are moving away from the plane do so. Let us suppose that the action between the molecule and the plane is the same as that between a perfectly elastic sphere and plane.

Let $m$ be the mass of an $A$ molecule, $v$ the velocity of the molecule, and $\alpha$ the angle its direction of motion makes with the normal to the plane before impact, $V$ the velocity after impact, $M$ the mass of the plane, $w$ and $W$ its velocity before and after it is struck by the molecule. Then we may easily show that

$$
\frac{\mathrm{I}}{2} m\left\{V^{2}-v^{2}\right\}=\frac{2 m M}{(M+m)^{2}}\left\{M w^{2}-m v^{2} \cos ^{2} \alpha-(M-m) v w \cos \alpha\right\} \text {. }
$$

Let us take the sum of the equations representing the effects of all the collisions which take place in unit time, we have

$$
\begin{aligned}
& \frac{1}{2} \Sigma m\left\{V^{2}-v^{2}\right\} \\
& =\frac{2 m M}{(M+m)^{2}} \Sigma\left\{M w^{2}-m v^{2} \cos ^{2} \alpha-(M-m) v w \cos \alpha\right\} \ldots(97) .
\end{aligned}
$$

If $N$ be the number of $A$ molecules which come in con- 
tact with the plane in unit time and $\theta_{1}$ the mean translatory kinetic energy of such molecules, then if $\delta \theta_{1}$ denotes the change in $\theta_{1}$ in unit time

$$
\frac{1}{2} \Sigma m\left(V^{2}-v^{2}\right)=N \delta \theta_{1}
$$

If $N^{\prime}$ be the number of collisions and $\theta$ the mean kinetic energy of the plane, then

$$
\Sigma M w w^{2}=2 N^{\prime} \theta .
$$

Since the directions of motion of the $A$ molecules are equally distributed

$$
\Sigma m v^{2} \cos ^{2} \alpha=\frac{1}{6} \Sigma_{m} m v^{2}=\frac{1}{3} N^{\prime} \theta_{1} .
$$

Since the plane is supposed to move so slowly that all the molecules moving towards it strike it, and since its average velocity is zero, we have

$$
\Sigma(M-m) w w \cos \alpha=0,
$$

so that equation (97) becomes

$$
N \delta \theta_{1}=\frac{2 M m}{(M+m)^{2}}\left\{2 N^{\prime} \theta-\frac{1}{3} N^{\prime} \theta_{1}\right\} \ldots . .(98) .
$$

If $\theta_{2}$ be the average translatory kinetic energy of the $B$ molecules which strike the plane in unit time, $N_{1}$ the number of such molecules and $N_{1}^{\prime}$ the number of collisions, $m^{\prime}$ the mass of a molecule, we have similarly

$$
N_{1} \delta \theta_{2}=\frac{2 M m^{\prime}}{\left(M+m^{\prime}\right)^{2}}\left\{2 N_{1}^{\prime} \theta-\frac{1}{3} N_{1}^{\prime} \theta_{2}\right\} \ldots . .(99),
$$

and we have also

$$
\begin{aligned}
& \delta \theta=-\frac{2 M m}{(M+m)^{2}}\left\{2 N^{\prime} \theta-\frac{1}{3} N^{\prime} \theta_{1}\right\} \\
& \quad-\frac{2 M m^{\prime}}{\left(M+m^{\prime}\right)^{2}}\left\{2 N_{1}^{\prime} \theta-\frac{1}{3} N_{1}^{\prime} \theta_{2}\right\} \ldots \ldots \ldots(\mathrm{I} 00) .
\end{aligned}
$$


Now we can make the average kinetic energy of the plane what we please by giving it the proper initial velocity. For our purpose we wish the plane to act as a transmitter and not as a storer of energy, and it will do so if we give it such an initial velocity that the mean kinetic energy of the plane does not alter in unit time. If this is the case $\delta \theta$ vanishes and we have by (100)

$$
\frac{m N^{\prime}}{(M+m)^{2}}\left\{2 \theta-\frac{1}{3} \theta_{1}\right\}+\frac{m^{\prime} N_{1}^{\prime}}{\left(M+m^{\prime}\right)^{2}}\left\{2 \theta-\frac{1}{3} \theta_{2}\right\}=0
$$

so that

if

$$
\left.\begin{array}{c}
2 \theta-\frac{1}{3} \theta_{1}=\frac{\frac{1}{3}\left(\theta_{2}-\theta_{1}\right) a}{a+b} \\
2 \theta-\frac{1}{3} \theta_{2}=-\frac{\frac{1}{3}\left(\theta_{2}-\theta_{1}\right) b}{a+b}
\end{array}\right\},
$$

Substituting these values for $2 \theta-\theta_{1} / 3$, and $2 \theta-\theta_{2} / 3$ in equations (98) and (99), we have

$$
\left.\begin{array}{c}
N \delta \theta_{1}=\frac{2 M a b}{3(a+b)}\left\{\theta_{2}-\theta_{1}\right\} \\
N^{\prime} \delta \theta_{2}=-\frac{2 M a b}{3(a+b)}\left\{\theta_{2}-\theta_{1}\right)
\end{array}\right\} .
$$

Thus if $\theta_{2}$ is greater than $\theta_{1}, \theta_{1}$ will increase and $\theta_{2}$ will diminish, and vice versâ, and if $\theta_{1}$ is equal to $\theta_{2}$ they will remain equal; thus the mean translatory energy behaves in these respects exactly like temperature. There seems nothing in the above illustration to restrict it to the case of gases, and we should expect it would hold equally well for solids or liquids. 
46. We are thus led to assume that part of the kinetic energy of a system, whether that system be a portion of a solid, liquid or gas, is proportional to the temperature.

Let us denote this part of the kinetic energy by

$$
\frac{1}{2}\left\{(u u) \dot{u}^{3}+\ldots\right\} \text {, }
$$

where $u$ is a coordinate helping to fix the position or configuration of a molecule. We see at once that there is an essential difference between these coordinates and those we have hitherto been considering and which fix the geometrical, strain, electric and magnetic configuration of the system. We have these latter coordinates entirely under our control and subject to certain limitations imposed by the finite strength of materials, the strength of dielectrics, and magnetic saturation; we may make them take any value we please. We may therefore fitly call these coordinates controllable coordinates. It is quite different, on the other hand, with the coordinates fixing the separate moving parts of the systems whose kinetic energy constitutes the temperature of the body. We can it is true affect the average value of certain functions of a large number of these coordinates, but we have no control over the coordinates individually. We may therefore call these coordinates "unconstrainable" coordinates. Their fundamental property is that we can not oblige any individual coordinate to take any value which may be assigned. Since we have no power of dealing with individual molecules, the "controllable" coordinates must merely fix the position of a large number of molecules as a whole.

If the term

$$
\frac{1}{2}\left\{(u u) \dot{u}^{2}+\ldots\right\}
$$

involves any "controllable" coordinate $\phi$, then it is evident 
that this coordinate $\phi$ must enter as a factor into all the terms in the form expressed by the equation

$$
\left.\frac{1}{2}\left\{(u u) \dot{u}^{2}+\ldots\right\}=\frac{1}{2} f(\phi)\left\{(u u)^{\prime} u^{2}+\ldots\right\} \ldots \ldots \text { (1 } 0 \text { r }\right),
$$

where the coefficients $(u u)^{\prime}$ do not involve $\phi$ : otherwise the phenomeron would be influenced more by the motion of some particular molecule than by that of others. We shall assume that $\theta$, the temperature, is proportional to

that is that

$$
\frac{1}{2}\left\{(u u) \dot{u}^{2}+\ldots\right\} \text {, }
$$

$$
\theta=\frac{1}{2} C\left\{(u u) \dot{u}^{2}+\ldots\right\} \ldots \ldots \ldots \ldots(\text { I02 }),
$$

where $C$ does not involve any of the "controllable" coordinates which fix the configuration of the system.

47. We may conveniently divide the kinetic energy of a system into two parts, one depending on the motion of "unconstrainable" coordinates, which we shall denote by $T_{u}$, and we shall assume that this is proportional to the absolute temperature $\theta$, the other depending on the motion of the "controllable" coordinates, we shall denote by $T_{c}$, $T_{c}$ corresponds to what v. Helmholtz in his paper on "Die Thermodynamik chemischer Vorgänge" (Wissenschaftliche Abhandlungen, i1. p. 958) calls "die freie Energie." There will not be any terms in the kinetic energy involving the product of the velocities' of an "unconstrainable" and a "controllable" coordinate, otherwise the energy of the system would be altered by reversing the motion of all the "unconstrainable" coordinates.

Let us suppose that $\phi$ is a controllable coordinate which enters into the expression for that part of the kinetic energy which expresses the temperature, then if $\Phi$ be the external force of this type acting on the system we have by Lagrange's equations, $V$ being the potential energy, 


$$
\Phi=\frac{d}{d t} \frac{d T}{d \dot{\phi}}-\frac{d T}{d \phi}+\frac{d V}{d \phi} .
$$

Now

$$
T=T_{c}+T_{u}
$$

and

so that

$$
\frac{d T_{u}}{d \dot{\phi}}=0
$$

$$
\Phi=\frac{d}{d t} \frac{d T_{c}}{d \dot{\phi}}-\frac{d T_{c}}{d \phi}-\frac{d T_{n}}{d \phi}+\frac{d V}{d \phi} \ldots \ldots \ldots . . .(1 \circ 3) .
$$

Now by equation (1०3) $T_{u}$ is of the form

$$
\frac{1}{2} f(\phi)\left\{(u u)^{\prime} i^{2}+\ldots\right\},
$$

where $(u n)^{\prime}$ does not involve $\phi$, so that we have

and therefore

$$
\frac{d T_{u}}{d \phi}=\frac{f^{\prime}(\phi)}{f(\phi)} T_{u} \ldots \ldots \ldots \ldots \ldots(\mathrm{r} \circ 4)
$$

$$
\Phi=\frac{d}{d t} \frac{d T_{c}}{d \dot{\phi}}-\frac{d T_{c}}{d \phi}-\frac{f^{\prime}(\phi)}{f(\phi)} T_{u}+\frac{d V}{d \phi} \ldots \ldots(\mathrm{r} 05) ;
$$

differentiating this equation on the supposition that all the controllable coordinates are constant and that the only variable is the energy depending on the motion of "uncontrollable" coordinates, we have

$$
\frac{d \Phi}{d T_{\varkappa}}=-\frac{f^{\prime}(\phi)}{f(\phi)}
$$

and therefore by (104)

$$
\left.T_{u} \frac{d \Phi}{d T_{u}}=-\frac{d T_{u}}{d \phi} \ldots \ldots \ldots \ldots \ldots \text { (1 } 06\right) .
$$

48. Now let us suppose that energy is communicated to the system, partly by the action of the external forces on the "controllable" coordinates, and partly through the "unconstrainable" coordinates : let the quantity of work communicated in the latter way be $\delta Q$. If the motion of the 
"unconstrainable" coordinates is that which gives rise to the energy corresponding to temperature, $\delta Q$ may be regarded as a quaritity of heat communicated to the system.

We have by the Conservation of Energy, if $\phi$ denotes a "controllable" coordinate,

$$
\delta Q+\Sigma \Phi \delta \phi=\delta T_{c}+\delta T_{u}+\delta V \ldots \ldots \ldots\left(\mathrm{r} \circ_{7}\right) .
$$

Now

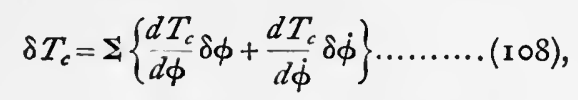

and since $T_{c}$ is a quadratic function of the velocities of the "controllable" coordinates, we have

and therefore

$$
2 T_{c}=\Sigma \dot{\boldsymbol{\phi}} \frac{d T_{c}}{d \dot{\phi}},
$$

$$
{ }_{2} \delta T_{c}=\Sigma\left\{\delta \dot{\phi} \frac{d T_{c}}{d \dot{\phi}}+\dot{\phi} \delta \frac{d T_{c}}{d \dot{\phi}}\right\} \ldots \ldots \ldots . .(\mathrm{r} \circ 9)
$$

so that by subtracting ( 108$)$ from (109) we get

$$
\delta T_{c}=\Sigma\left(\dot{\phi} \delta \frac{d T_{c}}{d \dot{\phi}}-\delta \phi \frac{d T_{c}}{d \phi}\right) \ldots \ldots \ldots \text { (I I } 0 \text { ). }
$$

Since the change in the configuration is that which actually takes place in the time $\delta t$, we have

so that

$$
\dot{\phi} \delta t=\delta \phi,
$$

$$
\delta T_{c}=\mathbf{\Sigma} \delta \phi\left\{\frac{d}{d t} \frac{d T_{c}}{d \dot{\phi}}-\frac{d T_{c}}{d \phi}\right\} \ldots \ldots \ldots(\mathrm{I} \mathrm{r}),
$$

and therefore equation (107) becomes

$$
\left.\delta Q=\Sigma \delta \phi\left\{\frac{d}{d t} \frac{d T_{c}}{d \dot{\phi}}-\frac{d T_{c}}{d \phi}-\Phi\right\}+\delta T_{u}+\delta V \ldots \ldots \text { (I I } 2\right) .
$$

Now, if $V$ be completely fixed by the controllable coordinates, we have

$$
\delta V=\Sigma \frac{d V}{d \phi} \delta \phi
$$

T. D. 
So that

$$
\delta Q=\Sigma \delta \phi\left\{\frac{d}{d t} \frac{d T_{c}}{d \phi}-\frac{d T_{c}}{d \phi}+\frac{d V}{d \phi}-\Phi\right\}+\delta T_{u} .
$$

Substituting for $\Phi$ the value given by (103) we have

$$
\delta Q=\Sigma \delta \phi \frac{d T_{u}}{d \phi}+\delta T_{u} \ldots \ldots \ldots \ldots . . . .\left(1 \mathrm{I}_{3}\right) ;
$$

but by (106)

$$
\frac{d T_{u}}{d \phi}=-T_{u}\left(\frac{d \Phi}{d T_{u}}\right)_{\phi \text { constant }},
$$

so that

$$
\delta Q=-\Sigma T_{u}\left(\frac{d \Phi}{d T_{u}}\right)_{\phi} \delta \phi+\delta T_{u} \ldots \ldots \ldots(\mathrm{II} 4) .
$$

Let us suppose that the quantity of work communicated to the system is just sufficient to prevent $T_{u}$ from changing, then

$$
\delta Q=-\Sigma T_{u}\left(\frac{d \Phi}{d T_{u}}\right)_{\phi} \delta \phi,
$$

or

$$
\left(\frac{d Q}{d \phi}\right)_{T_{u} \text { constant }}=-T_{u}\left(\frac{d \Phi}{d T_{u}}\right)_{\phi \text { constant }} \cdots \text { (I I 5). }
$$

Remembering that $T_{u}$ is proportional to the absolute temperature $\theta$, we see that equation ( $1 \mathrm{I}_{5}$ ) becomes

$$
\left(\frac{d Q}{d \phi}\right)_{\theta \text { constant }}=-\theta\left(\frac{d \Phi}{d \theta}\right)_{\phi \text { constant }} \ldots \ldots \ldots(\mathrm{I} \mathrm{I} 6),
$$

where in finding $d \Phi / d \theta$ we must take care that $\theta$ is the only quantity which varies.

In this form equation (II6) is identical with the third thermodynamical relation given in Maxwell's Theory of Heat, p. 169, and v. Helmholtz in his paper "Die Thermo- 
dynamik Chemischer Vörgange" (Wissenschaftliche Abhandlungen, 2, p. 962) deduces this equation from the Second Law of Thermodynamics and applies it to the case of the variation of the electromotive force of galvanic cells with temperature. The conclusions at which he arrives have been verified by the experiments of Czapski (Wied. Ann. 21, p. 209) and Jahn (Wied. Ann. 28, pp. 21, 49r). If $\delta Q=0$, that is if all the work done on the system is done by means of forces of the types of the various controllable coordinates, then we have by equation (II4)

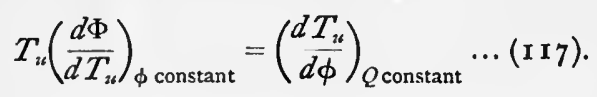

49. Since

$$
\frac{d T_{u}}{d \phi}=\frac{f^{\prime}(\phi)}{f(\phi)} T_{u}
$$

we see by (II3) that

$$
\delta Q=\Sigma \frac{f^{\prime}(\phi)}{f(\phi)} T_{u} \delta \phi+\delta T_{u},
$$

or

$$
\frac{\delta Q}{T_{u}}=\Sigma \delta \log f(\phi)+\delta \log T_{u} \ldots \ldots \ldots(\text { I I } 8),
$$

so that

$$
\frac{\delta Q}{T_{u}}
$$

is a perfect differential. This is analogous to the Second Law of Thermodynamics, and we see by the analogy that it shews that energy arising from the motion of quantities fixed by "unconstrainable" coordinates can only be partly converted into work spent in moving the quantities fixed by the "controllable" coordinates. The amount which can be converted follows laws analogous to those which regulate the conversion of heat into mechanical work. 
In the preceding work we have assumed that the potential energy of the system was not changed if the "controllable" coordinates remained unchanged. When however the system is a portion of a solid or liquid the potential energy may by some alteration in the state of aggregation be changed without there being any corresponding change in the controllable coordinates. To include this case we must suppose that $V$ is a function of the temperature as well as of the $\phi$ 's, and that its value in the neighbourhood of the temperature corresponding to a change of state in the substance varies very rapidly.

In this case we have $\theta$ being the temperature,

and instead of (I $\left.x_{4}\right)$

$$
\delta V=\frac{d V}{d \phi} \delta \phi+\frac{d V}{d \theta} \delta
$$

$$
\left.\delta Q=-\Sigma T_{u}\left(\frac{d \Phi}{d T_{u}}\right)_{\phi} \delta \phi+\delta T_{u}+\left(\frac{d V}{d \theta}\right)_{\phi} \delta \theta \ldots \text { (I I } 9\right) \text {. }
$$

Since $\delta \theta$ and $\delta T_{u}$ vanish together we see that equation (I I6) still holds. Equations $(\mathrm{I})$ ) and (II 8$)$ however require modification. We have now $\left(\delta Q-\delta V_{(\phi \text { constant })}\right) / T_{u}$ a perfect. differential instead of $\delta Q / T_{u t}$.

50. Relations between heat and strain. We shall now apply equation (II6) to determine the effects due to the variation of various physical quantities with temperature, and shall begin by considering the effects produced by the variation of the coefficients of elasticity $m$ and $n$ with temperature.

In equation (xi6) let us suppose that $\Phi$ is a stress of type $e$, then using the same notation as in $\S 35$, we have

$$
\begin{gathered}
\Phi=m(e+f+g)+n(e-f-g), \\
\left(\frac{d \Phi}{d \theta}\right)_{\phi \text { constant }}=\frac{d m}{d \theta}(e+f+g)+\frac{d n}{d \theta}(e-f-g) .
\end{gathered}
$$


So that by equation (I 6 ), $\delta Q$, the heat which must be supplied to unit volume of the bar to keep its temperature from changing when $e$ is increased by $\delta e$ is given by the equation

$$
\delta Q=-\left\{\frac{d m}{d \theta}(e+f+g)+\frac{d n}{d \theta}(e-f-g)\right\} \theta \delta e \ldots \ldots \text { (1 20), }
$$

and thus if the coefficients of elasticity diminish as the temperature increases, heat must be supplied to keep the temperature of a bar constant when it is lengthened, and hence if the bar is left to itself and not supplied with heat it will cool when it is extended.

If $\Phi$ is a couple tending to twist the bar about the axis of $x$, we have, if $a$ is the twist about that axis,

$$
\begin{aligned}
\Phi & =n a, \\
\frac{d \Phi}{d \theta} & =\frac{d n}{d \theta} a,
\end{aligned}
$$

and therefore by (II6) $\delta Q$, the heat required by unit volume of the bar to keep the temperature from changing when $a$ is increased by $\delta a$ is given by the equation

$$
\delta Q=-\theta \frac{d n}{d \theta} a \delta a \ldots \ldots \ldots \ldots \ldots(\mathrm{I} 2 \mathrm{I}),
$$

so that if a rod which is already twisted is twisted still further it will cool if left to itself, provided, as is usually the case, the coefficient of rigidity diminishes as the temperature increases.

The preceding results were first obtained by means of the Second Law of Thermodynamics by Sir William Thomson in his paper on the Dynamical Theory of Heat (Collected Papers, Vol. 1. p. 309).

5I. Thermal Effects produced by Electrification. Let us now consider the case when $\Phi$ is an electric 
force parallel to the axis of $x$, producing an electric displacement $f$ in that direction. In this case if $K$ be the specific inductive capacity of the dielectric, we have

$$
\begin{gathered}
\Phi={ }_{K}^{4 \pi} f, \\
\left(\frac{d \Phi}{d \theta}\right)_{f \text { constant }}=-\frac{4 \pi}{K^{2}} \frac{d K}{d \theta} f \ldots \ldots \ldots \ldots(\text { I 2 2) },
\end{gathered}
$$

so that $\delta Q$, the heat which must be supplied to unit volume: of the dielectric in order to prevent its temperature changing when the electric displacement is increased by $\delta f$, is by (I I6) given by the equation

$$
\left.\delta Q=\frac{4 \pi}{K^{2}} \frac{d K}{d^{2} \theta} \theta f \delta f \ldots \ldots \ldots \ldots \ldots \text { ( } 123\right) .
$$

Some recent experiments made by Mr Cassie in the Cavendish Laboratory on the effect of temperature on the specific inductive capacities of glass, mica and ebonite, have shewn that the specific inductive capacity of these dielectrics. increases as the temperature increases, and that at about $30^{\circ} \mathrm{C}$.

$$
\begin{aligned}
& \frac{\text { I }}{K} \frac{d K}{d \theta}=.002 \text { for glass, } \\
& \frac{1}{K} \frac{d K}{d \theta}=.0004 \text { for mica, } \\
& \frac{\mathrm{I}}{K} \frac{d K}{d \theta}=.0007 \text { for ebonite. }
\end{aligned}
$$

Thus the heat which must be supplied to unit volume of a piece of glass to enable its temperature to remain constant when it is electrified is by ( 123 )

$$
.002 \frac{2 \pi}{K} \cdot \theta f^{2}
$$

and this at $30^{\circ} \mathrm{C} . \quad=6 \frac{2 \pi f^{2}}{K}$; 
but

$$
\frac{2 \pi}{K} f^{2}
$$

is the work supplied from electrical sources, hence in charging a Leyden jar, we see that the mechanical equivalent of the heat absorbed by it during charging, if its temperature remains constant, is about two-thirds of the work supplied to it from electrical sources.

We see also by equation (123) that a piece of glass will be cooled when it moves from a place where the electric force is weak to one where it is strong.

52. Thermal effects of Magnetization. Let us now suppose that $\Phi$ is a magnetic force magnetizing a piece of soft iron or other magnetizable substance to the intensity $I$. Then if $k$ be the coefficient of magnetic induction

$$
\Phi=\frac{I}{k},
$$

so that

$$
\left(\frac{d \Phi}{d \theta}\right)_{I \text { constant }}=-\frac{I}{k^{2}} \frac{d k}{d \theta} \ldots \ldots \ldots \ldots(\mathrm{r} 24) .
$$

And therefore $\delta Q$ the heat which must be supplied to unit volume of the magnet to keep its temperature constant when the intensity of magnetization is increased by $\delta I$ is by equation (II6) given by the equation

$$
\delta Q=\theta \frac{I}{k^{2}} \frac{d k}{d \theta} \delta I \ldots \ldots \ldots \ldots \ldots(\text { I 25), }
$$

so that if the coefficient of magnetization decreases as the temperature increases then a magnet will get heated when its intensity of magnetization is increased, and therefore when it moves from weak to strong parts of the magnetic field. This was pointed out by Sir William Thomson in the paper just quoted. 
The experimental investigation of the heating effects produced by the motion of magnetizable bodies in variable magnetic fields is rendered difficult from the heating effect produced by the electric currents induced in the magnet by the alteration in the number of lines of magnetic force passing through it.

Another thing which would increase the difficulty is the phenomenon called by Ewing hysteresis (Experimental Investigation on Magnetism, Phil. Trans. I 885, p. I I). This causes the intensity of magnetization to depend not only on the strength of the magnetic force, but also on the previous magnetic history of the substance: so that the curve representing the relation between intensity of magnetization (ordinate) and magnetic force (abscissa), as the magnetic force goes through a complete cycle, will be of the kind shewn in the accompanying figure, and will enclose

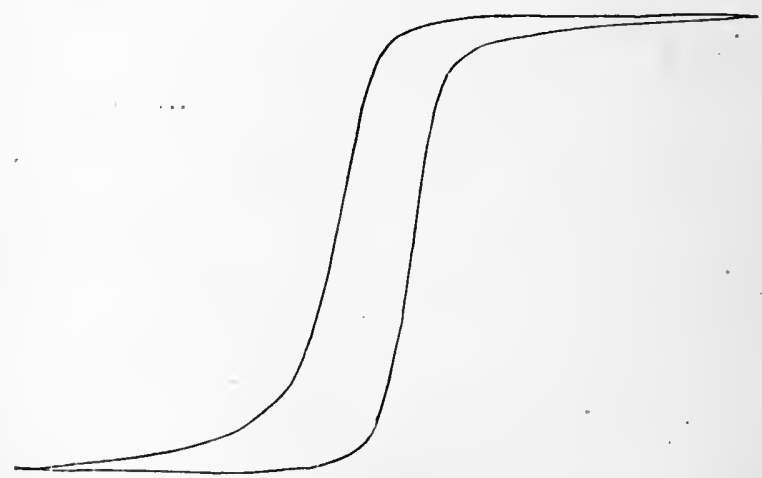

a finite area, indicating the dissipation of a finite quantity of energy proportional to the area of the curve, and this dissipated energy will appear as heat.

Experiments made on the effects of temperature upon 
the coefficient of magnetization of iron have shewn that these are rather complex. Baur (Wiedemann's Elektricität. iii. p. 750) from his experiments on this subject has arrived at the following results.

The influence of temperature upon the magnitude of the coefficient of magnetization depends upon the magnitude of the magnetizing force.

The coefficient of magnetization increases with the temperature if the magnetizing force does not exceed a certain value, but when the magnetizing force exceeds this value the coefficient of magnetization diminishes as the temperature increases.

The smaller the magnetizing force the greater the influence of temperature upon the coefficient of magnetization.

Taking these results in conjunction with equation (125) we see,

I. That when a magnetizable body moves in a magnetic field where the force is everywhere less than the critical value, its temperature will tend to fall when it moves from places of weak to places of strong magnetic force and vice versâ.

2. That when the body is placed in a magnetic field where the magnetic force is everywhere greater than the critical value, its temperature will rise when it moves from places of weak to places of strong magnetic force and vice versâ.

The coefficient of magnetization of nickel diminishes as the temperature increases, so that a piece of nickel will get warmer when it moves from a weak to a strong part of the magnetic field. The coefficient of magnetization of cobalt on the other hand increases as the temperature increases, so that a piece of cobalt will cool as it moves from a weak to a strong part of the magnetic field. 


\section{CHAPTER VII.}

\section{ELECTROMOTIVE FORCES DUE TO DIFFERENCES OF TEMPERATURE.}

53. WE shall now go on to consider various cases in which inequalities of temperature in a substance give rise to electromotive forces.

Sir William Thomson has shewn that when a current of electricity flows along an unequally heated bar it carries with it as it flows from a hot to a cold place either heat or cold : heat if the bar is made of brass or copper, cold if it is made of iron. Sir William Thomson expressed this result by saying that the specific heat of electricity in copper and brass is positive, since the electricity in this case carries heat with it just as if it were a real fluid possessing specific heat ; the "specific heat" of electricity in iron on the other hand is said to be negative, since electricity in iron behaves with regard to heat in the opposite way to a fluid possessing specific heat.

It follows from this result, by the consideration of the reciprocal relations, that electromotive forces must be developed in any conductor the temperature of which is not uniform throughout. We shall now endeavour to find what terms in the Lagrangian function these phenomena corre- 
spond to, or rather we shall shew that if there was a certain term in the Lagrangian function an unequally heated body would exhibit similar phenomena.

Let us suppose that in the term

$$
\frac{1}{2}\left\{(u u) \ddot{u}^{2}+\ldots\right\},
$$

which expresses the part of the kinetic energy of unit volume of the substance due to sensible heat, the coeffcients $(u u)$ are functions of

$$
\frac{d}{d x}\left(\sigma_{x} f\right)+\frac{d}{d y}\left(\sigma_{y} g\right)+\frac{d}{d z}\left(\sigma_{z} h\right),
$$

where $\sigma_{x}, \sigma_{y}, \sigma_{z}$ are quantities not explicitly involving. $f, g, h$, the quantities of electricity which have passed through planes of unit area at right angles to the axes. $x, y, z$ respectively

Let us write for the sake of brevity

$$
\frac{d}{d x}\left(\sigma_{x} f\right)+\frac{d}{d y}\left(\sigma_{y} g\right)+\frac{d}{d z}\left(\sigma_{z} h\right) \equiv \epsilon .
$$

Then, since $f, g, h$ are controllable coordinates, and by hypothesis (uul) involves $\epsilon$, we may write

$$
\frac{1}{2}\left\{(u u) \dot{u}^{2}+\ldots\right\}=f(\epsilon) \frac{1}{2}\left\{(u u)^{\prime} \dot{u}^{2}+\ldots\right\},
$$

where $f(\epsilon)$ denotes some function of $\epsilon$. The coefficients. $(u u)^{\prime}$ are supposed not to involve $f, g, h$ explicitly.

By Hamilton's principle any term in the Lagrangian function indicates the existence of effects which are the same as those which would be produced by electromotive forces parallel to the axes of $x, y, z$, and equal to the coefficients of $\delta f, \delta g, \delta / \imath$ which this term contributes when the variation of the Lagrangian function is taken.

The term we are considering is, taking the whole volume,

$$
\iiint f(\epsilon) \frac{1}{2}\left\{(u u \iota)^{\prime} \ddot{u}^{2}+\ldots\right\} d x d y d z \text {. }
$$


When $\epsilon$ is increased by $\delta \epsilon$ the alteration in this term is

$$
\frac{1}{2} \iiint \delta \epsilon f^{\prime}(\epsilon)\left\{(u u)^{\prime} \dot{u}^{2}+\ldots\right\} d x d y d z \ldots \ldots \text { (1 26). }
$$

Since

$$
\delta \epsilon=\frac{d}{d x}\left(\sigma_{x} \delta f\right)+\frac{d}{d y}\left(\sigma_{y} \delta g\right)+\frac{d}{d z}\left(\sigma_{z} \delta h\right),
$$

we see that if we integrate (126) by parts the terms in $\delta f$ are

$$
\begin{aligned}
& \frac{1}{2} \iint \sigma_{x} f^{\prime}(\epsilon)\left\{(u u)^{\prime} \dot{u}^{2}+\ldots\right\} \delta f d y d z \\
& \quad-\frac{1}{2} \iiint \sigma_{x} \frac{d}{d x}\left\{f^{\prime}(\epsilon)\left[(u u)^{\prime} u^{2}+\ldots\right]\right\} \delta f d x d y d z \ldots(\text { I 2 } 7) .
\end{aligned}
$$

Since

$$
\frac{1}{2} f(\epsilon)\left\{(u u t)^{\prime} \dot{u}^{2}+\ldots\right\}
$$

is proportional to the temperature, we may put

$$
\frac{1}{2} f(\epsilon)\left\{(u u)^{\prime} \ddot{u}^{2}+\ldots\right\}=\beta \theta,
$$

and then (127) may be written

$$
\iint \sigma_{x} \frac{f^{\prime}(\epsilon)}{f(\epsilon)} \beta \theta \delta f d y d z-\iiint \sigma_{x} \frac{d}{d x}\left\{\frac{f^{\prime}(\epsilon)}{f(\epsilon)} \beta \theta\right\} \delta f d x d y d z .
$$

So that by Hamilton's principle if $X$ be the force per unit length which would produce the same effects as this term indicates

$$
X=-\sigma_{x} \frac{d}{d x}\left\{\frac{f^{\prime}(\epsilon)}{f(\epsilon)} \beta \theta\right\} .
$$

To take the simplest case let us suppose that $f(\epsilon)$ is a dinear function of $\epsilon$, so that

and

$$
\begin{aligned}
f(\epsilon) & =a+b \epsilon, \\
\frac{f^{\prime}(\epsilon)}{f(\epsilon)} & =\frac{b}{a+b \epsilon} .
\end{aligned}
$$

As $b \epsilon / a$ is the alteration in the energy made by the electrification, it can only be a small quantity, so that we 
have approximately

$$
\frac{f^{\prime}(\epsilon)}{f(\epsilon)}=\frac{b}{a}
$$

and therefore

$$
X=-\sigma_{x} \beta \frac{d}{d x}\left(\frac{\mathrm{I}}{a} b \theta\right) \ldots \ldots \ldots \ldots(\mathrm{I} 28),
$$

or if $b$ and $a$ remain constant throughout the substance,

$$
X=-\sigma_{x} \frac{b \beta}{a} \frac{d \theta}{d x}
$$

So that this term indicates the existence of an electromotive force parallel to $x$ and proportional to the rate of alteration of the temperature in that direction. If $Y$ and $Z$ are the electromotive forces parallel to $y$ and $z$ respectively we have

$$
\left.\begin{array}{l}
Y=-\sigma_{y} \frac{b \beta}{a} \frac{d \theta}{d y} \\
Z=-\sigma_{z} \frac{b \beta}{a} \frac{d \theta}{d z}
\end{array}\right\} .
$$

The occurrence of $\delta f$ in the surface integral shews that there is a discontinuity in the potential at the surface of separation of two media and that the potential in the first medium is higher than that in the second by

$$
\left\{\left(\frac{\sigma_{x} b \beta}{a}\right)_{2}-\left(\frac{\sigma_{x} b \beta}{a}\right)_{1}\right\} \theta,
$$

where the suffix attached to the bracket indicates the medium for which the value of the quantity inside the bracket is to be taken.

54. Thermal effects of this term. Let us suppose that $\delta Q$ is the quantity of heat that must be supplied to unit volume of the conductor to keep its temperature from changing when a quantity of electricity $\delta f$ flows through it, that is when $f$ is increased by $\delta f$. 
We see by equation ( $\mathrm{II}_{3}$ ) that

$$
\begin{aligned}
\delta Q & =\text { the increase in } T_{w} \text { when } f \text { is increased by } \delta f \\
& =-\sigma_{x} \frac{b \beta}{a} \frac{d \theta}{d x} \delta f \text { by equations } 127 \text { and } \mathbf{1 2 9} .
\end{aligned}
$$

If $u$ be the current parallel to $x$ and $\delta t$ the time it has been flowing

so that

$$
\begin{gathered}
\delta f=u \delta t, \\
\delta Q=-\sigma_{x} \frac{b \beta}{a} \frac{d \theta}{d x} u \delta t \ldots \ldots . . . . . .(\text { ( зо }) .
\end{gathered}
$$

If the current flows in the direction in which heat is flowing, that is from hot to cold, $\delta Q$ will have the same sign as $\sigma_{x} b$, since $\beta$ and $u$ are necessarily positive. Hence if $\sigma_{x} b$ is positive heat must be supplied to unit volume to keep the temperature constant when a current flows into it from a hotter place, that is a current from a hot to a cold place carries cold with it, so that in this case the electricity behaves as if it had a negative specific heat. Hence $\sigma_{x} b$ is of the opposite sign to the specific heat of electricity in the substance.

We see from equation ( 130 ) that the electromotive force at any part of the circuit always tends to produce a current in the same direction as one which would cause a fall in temperature at this part of the circuit.

If we produced a distribution of electricity throughout the volume of a body, some very peculiar results would follow if this term existed.

Let us take the case of an isotropic body whose temperature is uniform, then we may suppose that $\sigma_{x}, \sigma_{y}$, $\sigma_{z}$ are each equal to $\sigma$ and independent of $x, y, z$, then

$$
\frac{d}{d x}\left(\sigma_{x} f\right)+\frac{d}{d y}\left(\sigma_{y} g\right)+\frac{d}{d z}\left(\sigma_{z} h\right)=\sigma\left\{\frac{d f}{d x}+\frac{d g}{d y}+\frac{d h}{d z}\right\} ;
$$


but if $\rho$ be the volume density of the distribution of electricity,

$$
\frac{d f}{d x}+\frac{d g}{d y}+\frac{d h}{d z}=-\rho .
$$

So that the energy in unit volume corresponding to the heat energy equals

$$
\frac{1}{2}\{a-b \sigma \rho\}\left\{(u u)^{\prime} \dot{u}^{2}+\ldots\right\} \ldots \ldots \ldots \ldots(\text { I } 3 \mathrm{I}),
$$

and thus when we alter the volume density of the electricity we alter the energy due to the heat and therefore the temperature.

To calculate the amount and even the sign of this alteration in temperature we must observe that $\dot{u} \ldots$ will be altered if we suddenly alter $\rho$. The case is quite analogous to that of a moving body the effective mass of which is suddenly increased, we may suppose, by the tightening of a string attached to another mass. In this case it is the momentum of the system and not its velocity which remains constant.

If we express the term ( $13 \mathbf{r})$ in terms of the momenta $v_{1}, v_{2} \ldots$ corresponding to the various coordinates $u_{1}, u_{2}$, we see, since

$$
v_{1}=\frac{d T}{d \dot{u}_{1}},
$$

that it will be of the form

$$
\frac{\mathrm{I}}{a-b \sigma \rho} f\left(v_{1}, v_{2} \ldots\right)
$$

where $f\left(v_{1}, v_{2} \ldots\right)$ denotes a quadratic function of $v_{1}, v_{2}$ $\&$ c:, which does not involve $\rho$. As this expression is proportional to the temperature $\theta$, we see that if $\rho$ be suddenly increased by $\delta \rho$, the increase $\delta \theta$ in the temperature is given 
by the equation

$$
\frac{\delta \theta}{\theta}=\frac{b \sigma \delta \rho}{a-b \sigma \rho} \ldots \ldots \ldots \ldots \ldots \ldots\left(\mathrm{I} 3^{2}\right),
$$

so that if $b \sigma$ is positive the temperature of the body is increased by communicating a charge of electricity to it, that is the electricity behaves like a body whose specific heat was negative. But we saw that $b \sigma$ was of the opposite sign to what Sir William Thomson has defined as the specific heat of electricity in the substance. Hence we see that the analogy between the behaviour of electricity and that of a fluid possessing either positive or negative specific heat can be extended to cover the case when a bodily charge of electricity is communicated to the body.

We can shew however that if the charge of electricity be of the same order of magnitude as those which occur in electrostatic phenomena this heating effect must be extremely small. For multiplying both sides of equation (132) by $\beta$, we have

$$
\frac{\beta \delta \theta}{\theta}=\frac{\beta b \sigma}{a} \delta \rho, \text { approximately. }
$$

Now $\beta b \sigma / a$ is by equation ( $13^{\circ}$ ) the "specific heat" of electricity. The value of this for antimony at the temperature $27^{\circ} \mathrm{C}$. is (see Tait's Heat, p. 180) about $10^{-2} \times 300$ when the unit is $10^{-5}$ of the E. M. F. of a Grove's cell. As the E. M. F. of a Grove's cell is about $2 \times 10^{8}$ in absolute measure the "specific heat" of electricity in antimony in absolute measure will be about 6000 .

We must now find a limiting value for $\delta \rho$. Let us suppose that electricity is uniformly distributed through a sphere of radius $r$, then if $\rho$ be the density of the electrical distribu- 
tion, $K$ the specific inductive capacity, the force just outside the sphere is

$$
\frac{4}{3 K} \pi \rho r \text {. }
$$

Now the greatest value this can have in air is (see Everett's Units and Physical Constants, p. 142) about $4 \times 10^{12}$, so that a limiting value of $\rho$ will be given by

$$
\frac{4}{3 K} \pi \rho r=4 \times 10^{12} \text {. }
$$

Now

$$
K=\frac{\mathrm{I}}{9 \times 10^{20}}
$$

so that

$$
\rho=\frac{\mathrm{I}}{9 \times 10^{8} r} \text { approximately. }
$$

Hence substituting this value of $\rho$ for $\delta \rho$, we get at the temperature $27^{\circ} \mathrm{C}$.

$$
\begin{aligned}
\beta \delta \theta & =\frac{300 \times 6 \times 10^{3}}{9 \times 10^{8} \times r} \\
& =\frac{2}{10^{3} r} .
\end{aligned}
$$

Now $\beta \delta \theta$ is the mechanical equivalent of the heat available for changing the temperature, so that the change in temperature will be of the order

$$
\frac{\mathrm{I}}{2 . \mathrm{I} \times 10^{10}} \frac{\mathrm{I}}{r},
$$

since $4^{.2} \times 10^{7}$ is the mechanical equivalent of heat. Thus the change of temperature which can be produced in this way by any statical charge of electricity is infinitesimal.

55. Thermoelectric effects of strain. If the quantity $b$ in the expression for $f(\epsilon)$ is a function of the strain in the wire along which the current is passing, then putting $b=f(e)$, where $e$ denotes a strain in the wire, we

T. D. 
see by equation (128) that at each point of the wire there is the electromotive force

$$
-\sigma_{x} \frac{d}{d s}\left\{\frac{\mathrm{I}}{a} f(e) \beta \theta\right\},
$$

acting along the wire, $d s$ being an element of its length.

Now if we have a closed circuit made of one metal, in which $\sigma_{x}$ may vary with the temperature and state of strain, then the integral of the expression taken round the circuit will vanish if either $\theta$ or $e$ is constant all along the circuit but will not in general vanish if both $\theta$ and $e$ vary round the circuit. So that we cannot produce currents in a wire whose temperature is constant by any variation in the strain, nor in a wire where the strain is constant by any variation in the temperature, while on the other hand we should expect to get currents if both the strain and the temperature were variable. All these results agree exactly with experiment, and hence we are led to conclude that $b$ is a function of the strain.

If this is so then communicating a volume distribution of electricity to an unequally heated rod must tend to strain it.

For let us suppose that the strain $e$ is an extension of a wire, then if $a$ be the displacement of a point along the wire

$$
e=\frac{d \alpha}{d s} .
$$

If $a$ be increased by $\delta a$, the coefficient of $\delta a$ in the change in the Lagrangian function is, when the medium is isotropic

$$
-\frac{d}{d s}\left\{\frac{\mathrm{I}}{a} f^{\prime}(e) \sigma \rho \theta\right\}
$$

and therefore, by Hamilton's principle, the effects due to 
this term are the same as would be produced by an external force equal per unit length to (r33) tending to strain the wire.

Thus when an unequally heated wire has electricity distributed throughout the volume there will be stresses tending to strain the wire.

If we consider twist instead of elongation we can show in a similar way that an unequally lieated wire will be twisted when electricity is distributed through it.

56. The electromotive force in a thermoelectric circuit is generally calculated from the heat developed in various parts of the circuit by the passage of the current. The amount of knowledge of the electromotive force which we can derive from thermal considerations is however limited in a way which $\mathrm{I}$ think is generally overlooked.

We see by $\S 47$ that when a coordinate $x$ is increased by $\delta x$, the heat $\delta Q$ which must be supplied to the system to prevent its temperature from changing is given by the equation

$$
\delta Q=-\frac{d X}{d \theta} \delta x
$$

where $X$ is the force of type $x$ acting on the system.

Now let $X$ be an electromotive force in a thermoelectric circuit and $x$ a quantity of electricity, then we see by (I I4) that from considerations about the heat developed we can only derive information about the part of the electromotive force which depends upon temperature and cannot tell anything whatever about any other part.

As a particular application of this principle we see that the Peltier effect can throw no light on the absolute difference of potential between two different metals and hence there is nothing in the phenomena of thermoelectricity to force us to attribute the large difference in potential ob- 
served by Volta between two different metals in contact to chemical action between them and the surrounding. medium.

57. Electromotive forces produced by inequalities of temperature in a magnetic field. v. Ettinghausen and Nernst (Wiedemann's Annalen, xxxi. 737 and. 760,1887 ) have recently discovered an electromotive force due to inequalities in temperature which is very analogous to the Hall effect. They found that when heat is flowing across a thin plate made of a substance which can conduct electricity, electromotive forces are produced in the plate, if it is placed in a magnetic field. The direction of the electromotive force is at right angles both to the magnetic force and to the direction in which the temperature is changing fastest. The magnitude of the electromotive force is proportional to the product of the magnetic force into the rate of increase of the temperature at right angles to the lines of magnetic force.

This electromotive force is especially large in bismuth.

If $\theta$ represents the temperature, and $\alpha, \beta, \gamma$ the components of magnetic force parallel to the axes of $x, y, z$ respectively, the components of the electromotive force due to this effect will if the laws we have just quoted are true be given by the expressions

$$
\begin{aligned}
& Q\left\{\gamma \frac{d \theta}{d y}-\beta \frac{d \theta}{d z}\right\}, \\
& Q\left\{\alpha \frac{d \theta}{d z}-\gamma \frac{d \theta}{d x}\right\}, \\
& Q\left\{\beta \frac{d \theta}{d x}-\alpha \frac{d \theta}{d y}\right\},
\end{aligned}
$$

where $Q$ is a quantity which has very different values in different substances. The results of Nernst's determinations 
ELECTROMOTIVE FORCE IN MAGNETIC FIELD. II of this quantity (Wied. Ann. xxxI. 775), are given in the following table

\begin{tabular}{l|c}
\hline & $Q$ \\
\hline Bismuth & $-\cdot 132$ \\
Antimony & -.00887 \\
Nickel & $-.0086 \mathrm{r}$ \\
Cobalt & -.00224 \\
Iron & +.00156 \\
Steel & +.000706 \\
Copper & +.000090 \\
Zinc & +.000054 \\
Silver & +.000046
\end{tabular}

We shall now proceed to see what term in the Lagrangian function would give rise to forces of this kind.

Let us consider the term

$$
\begin{aligned}
\iiint \theta\left\{\frac{d}{d x}\left[Q\left(\gamma_{o}^{g}-\beta h\right)\right]+\right. & \frac{d}{d y}[Q(\alpha h-\gamma f)] \\
& \left.+\frac{d}{d z}[Q(\beta f-\alpha g)]\right\} d x d y d z,
\end{aligned}
$$

where $f, g, h$ are the components of the electric displacement parallel to the axes of $x, y, z$ respectively.

The variation of this term when $f$ is increased by $\delta f$ is

$$
\iint \delta f Q \theta(\beta n-\gamma m) d S-\iiint \delta f Q\left(\beta \frac{d \theta}{d z}-\gamma \frac{d \theta}{d y}\right) d x d y d z,
$$

where $l, m, n$ are the direction cosines drawn outwards of the normal to the surface enclosing the volume through which the integrals are extended.

By Hamilton's principle the term in the surface integral indicates that if we draw any circuit in the field then when this circuit crosses the boundary of two media there is an 
electromotive force whose components parallel to the axes of $x, y, z$ are respectively

$$
\begin{aligned}
& \theta\left[n\left\{Q_{2} \beta_{2}-Q_{1} \beta_{1}\right\}-m\left\{Q_{2} \gamma_{2}-Q_{1} \gamma_{1}\right\}\right] \\
& \theta\left[l\left\{Q_{2} \gamma_{2}-Q_{1} \gamma_{1}\right\}-n\left\{Q_{2} \alpha_{2}-Q_{1} \alpha_{1}\right\}\right] \\
& \theta\left[m\left\{Q_{2} \alpha_{2}-Q_{1} \alpha_{1}\right\}-l\left\{Q_{2} \beta_{2}-Q_{1} \alpha_{1}\right\}\right],
\end{aligned}
$$

where corresponding quantities in the media (r) and ( 2 ) are indicated by affixing the suffixes $I$ and 2 respectively to the symbol representing the quantity, and $l, m, n$ are the direction cosines of the normal drawn from medium (2) to medium (I).

By the same principle the terms in the volume integral indicate the existence of an electromotive force throughout the body whose components per unit length parallel to the axes of $x, y, z$ are

$$
\left.\begin{array}{l}
Q\left(\gamma \frac{d \theta}{d y}-\beta \frac{d \theta}{d z}\right) \\
Q\left(\alpha \frac{d \theta}{d z}-\gamma \frac{d \theta}{d x}\right) \\
Q\left(\beta \frac{d \theta}{d x}-\alpha \frac{d \theta}{d y}\right)
\end{array}\right\}
$$

these are the expressions for the components of the electromotive force discovered by $\mathrm{v}$. Ettinghausen and Nernst.

These forces do not satisfy the solenoidal condition; they will therefore produce a distribution of electricity throughout the substance whose volume density $\rho$ is given by the equation

$$
\begin{aligned}
\rho=\frac{K}{4 \pi}\left[\frac{d}{d x} Q\left\{\gamma \frac{d \theta}{d y}-\beta \frac{d \theta}{d z}\right\}+\frac{d}{d y} Q\right. & \left\{\alpha \frac{d \theta}{d z}-\gamma \frac{d \theta}{d x}\right\} \\
& \left.+\frac{d}{d z} Q\left\{\beta \frac{d \theta}{d x}-\alpha \frac{d \theta}{d y}\right\}\right],
\end{aligned}
$$

where $K$ is the specific inductive capacity of the substance, thus, 


$$
\rho=\frac{K Q}{4 \pi}\left\{\begin{array}{l}
d \theta \\
d x
\end{array}\left(\frac{d \beta}{d z}-\frac{d \gamma}{d y}\right)+\frac{d \theta}{d y}\left(\frac{d \gamma}{d x}-\frac{d \alpha}{d z}\right)+\frac{d \theta}{d z}\left(\frac{d \alpha}{d y}-\frac{d \beta}{d x}\right)\right\},
$$

or neglecting $Q^{2}$

$$
\rho=-K Q\left\{u \frac{d \theta}{d x} \div v^{\prime} \frac{d \theta}{d y}+\imath^{\prime} \frac{d \theta}{d z}\right\}
$$

where $u, v, w$ are the components of the current.

58. Thermal phenomena arising from this term. We can see by equation ( $\mathrm{Ir}_{3}$ ) that $\delta H$ the heat required by unit volume to prevent the temperature from changing when a quantity of electricity $\delta f$ passes through it parallel to the axis of $x$ is given by the equation

$$
\begin{gathered}
\delta H=\text { (that part of the electromotive force which } \\
\text { arises from the part of the energy corre- } \\
\text { sponding to the sensible heat) } \delta f ;
\end{gathered}
$$

thus the part of $\delta H$ which arises from this term is given by

$$
\delta H=Q\left(\gamma \frac{d \theta}{d y}-\beta \frac{d \theta}{d z}\right) \delta f
$$

so that when quantities of electricity $\delta f, \delta g, \delta /$ pass parallel to the axes of $x, y, z$ respectively then

$$
\delta H=Q\left\{\left(\gamma \frac{d \theta}{d y}-\beta \frac{d \theta}{d z}\right) \delta f+\left(\alpha \frac{d \theta}{d z}-\gamma \frac{d \theta}{d x}\right) \delta g+\left(\beta \frac{d \theta}{d x}-\alpha \frac{d \theta}{d y}\right) \delta /\right\}
$$

or, if $u, v, v$ are the components of current parallel to the axes of $x, y, z$ respectively and $\delta t$ the time the displacement takes, then since

$$
\delta f=u \delta t ; \delta g=v \delta t ; \delta /=w \delta t
$$

we have

$$
\begin{aligned}
\delta H & =Q \delta t\left[\left\{\gamma \frac{d \theta}{d y}-\beta \frac{d \theta}{d z}\right\} u+\left\{\alpha \frac{d \theta}{d z}-\gamma \frac{d \theta}{d x}\right\} v+\left\{\beta \frac{d \theta}{d x}-\alpha \frac{d \theta}{d y}\right\} w^{\prime}\right] \\
& =Q \delta t\left[\frac{d \theta}{d x}\{\beta w-\gamma v\}+\frac{d \theta}{d y}\{\gamma u-\alpha w\}+\frac{d \theta}{d z}\left\{\alpha v-\beta \mho^{\prime}\right\}\right] \text { (I35). }
\end{aligned}
$$


If $X, Y, Z$ are the mechanical forces acting on unit volume of the conductor arising from the action of the magnetic field on the currents flowing through the volume

$$
\begin{aligned}
& X=\mu\{\gamma v-\beta w\} \\
& Y=\mu\{a z-\gamma u\} \\
& Z=\mu\{\beta u-a v\},
\end{aligned}
$$

where $\mu$ is the magnetic permeability, combining these equations with ( 135 ) we see that

$$
\delta H=-\frac{\mathbf{I}}{\mu} Q \delta t\left\{X \frac{d \theta}{d x}+Y \frac{d \theta}{d y}+Z \frac{d \theta}{d z}\right\} .
$$

So that if the action of the mechanical force on the current tends to make the substance conveying the current move in the direction in which heat is flowing, then when $Q$ is negative, heat must be abstracted from the substance to keep its temperature constant when currents of electricity flow through it. And the heat which has to be supplied in unit time to unit volume to prevent the temperature from changing is given by the equation

$$
\begin{gathered}
\delta H=\frac{\mathbf{I}}{\mu} Q \quad \begin{array}{l}
\text { \{resultant mechanical force on } \\
\text { unit volume } \times \text { flow of heat } \times \\
\text { cosine of the angle between } \\
\text { these two quantities\}. }
\end{array}
\end{gathered}
$$

Heating effects in a magnetic field have been detected by v. Ettinghausen (Wiedemann's Annalen, xxx. pp. 737$760,1887)$.

59. Magnetic effects of this term. If we apply to the term

$$
\iiint \theta\left[\frac{d}{d x}\left\{Q\left(\gamma^{g}-\beta h\right)\right\}+\frac{d}{d y}\{Q(\alpha h-\gamma f)\}+\frac{d}{d z}\{Q(\beta f-\alpha g)\}\right]
$$


the same method as the one which in $\S 43$ we applied to the term corresponding to Hall's effect, we shall see that it involves the existence at a point $\xi, \eta$, $\zeta$ where there are no electric displacements of a magnetic force whose components parallel to the axes of $x, y, z$ are respectively

$$
-\frac{d \chi}{d \xi},-\frac{d \chi}{d \eta},-\frac{d \chi}{d \zeta}
$$

where

$$
\begin{aligned}
\chi=-\iiint Q^{\theta}\left[\left(\frac{d g}{d z}-\frac{d h}{d y}\right) \frac{d}{d x} \frac{\mathrm{I}}{r}\right. & +\left(\frac{d h}{d x}-\frac{d f}{d z}\right) \frac{d}{d y} \frac{\mathrm{I}}{r} \\
& \left.+\left(\frac{d f}{d y}-\frac{d g}{d x}\right) \frac{d}{d z} \frac{\mathrm{I}}{r}\right] d x d y d z,
\end{aligned}
$$

where $r$ is the distance between the points $x, y, z$ and $\xi, \eta, \zeta$.

If $\xi, \eta, \zeta$ is a point at which there is an electric displacement, then as before the components of the magnetic force parallel to $x, y, z$ respectively are

$$
\left.\begin{array}{l}
-\frac{d \chi}{d \xi}-\frac{4 \pi}{3} Q\left(h \frac{d \theta}{d \eta}-g \frac{d \theta}{d \zeta}\right) \\
-\frac{d \chi}{d \eta}-\frac{4 \pi}{3} Q\left(f \frac{d \theta}{d \zeta}-h \frac{d \theta}{d \xi}\right) \\
-\frac{d \chi}{d \zeta}-\frac{4 \pi}{3} Q\left(g \frac{d \theta}{d \xi}-f \frac{d \theta}{d \eta}\right)
\end{array}\right\}
$$

We see that the equation

$$
4 \pi u=\frac{d \gamma}{d \eta}-\frac{d \beta}{d \zeta}
$$

is no longer true, but that now

$$
\begin{aligned}
& 4 \pi u-\frac{4 \pi}{3} Q\left\{\frac{d \theta}{d \xi}\left(\frac{d f}{d \xi}+\frac{d g}{d \eta}+\frac{d h}{d \zeta}\right)-\frac{d f}{d \xi} \frac{d \theta}{d \xi}-\frac{d f}{d \eta} \frac{d \theta}{d \eta}-\frac{d f}{d \zeta} \frac{d \theta}{d \zeta}\right\} \\
& =\frac{d \gamma}{d \eta}-\frac{d \beta}{d \zeta} \text {. }
\end{aligned}
$$


It follows from equation ( 136 ) that if dielectrics as well as conductors exhibit the phenomenon discovered by $v$. Ettinghausen and Nernst then a steady electric displacement through a heated dielectric may produce magnetic forces. A numerical calculation similar to that in $\S 43$ will show however that these forces are exceedingly small.

6o. Thermal effects accompanying changes in magnetization, arising from this term. Since the magnetic forces expressed by equation (136) arise from that part of the kinetic energy which corresponds to the sensible heat, changes in the intensity of magnetization must by equation (II4) be accompanied by reversible thermal effects. If the intensities parallel to the axes of $x, y, z$ be increased by $\delta A, \delta B, \delta C$ respectively, then by equation (I 14) $\delta H$ the mechanical equivalent of the heat which must be supplied to unit volume to prevent its temperature from changing is given by the equation

$$
\begin{aligned}
\delta H=-\frac{4}{3} \pi Q\left\{\left(h \frac{d \theta}{d \eta}-g \frac{d \theta}{d \zeta}\right) \delta A+(\right. & \left.f \frac{d \theta}{d \zeta}-h \frac{d \theta}{d \xi}\right) \delta B \\
& \left.+\left(g \frac{d \theta}{d \xi}-f \frac{d \theta}{d \eta}\right) \delta C\right\} .
\end{aligned}
$$

61. Rotation of the plane of polarization produced by the flow of heat. Rowland has shown that if Hall's effect exists in dielectrics, then, according to Maxwell's Electromagnetic Theory of Light, the plane of polarization of plane polarized light will be rotated when the dielectric through which the light is passing is placed in a magnetic field the lines of force of which are more or less parallel to the direction of propagation of the light. We shall now proceed to investigate whether the existence of v. Ettinghausen's and Nernst's phenomenon will produce 
a rotation of the plane of polarization when a ray of plane polarized light passes through a dielectric through which heat is flowing.

Let us suppose that we have a circularly polarized ray of light travelling parallel to the axis of $z$ through a dielectric in which there is a uniform flow of heat also parallel to $z$.

Let $f$ and $g$ be the electric displacements parallel to the axes of $x$ and $y$ respectively, $F$ and $G$ the components of the vector potential parallel to $x$ and $y$ respectively, and $X$ and $Y$ the components of the electromotive force parallel to these axes, then since $d \theta / d x, d \theta / d y$ both vanish

$$
\begin{aligned}
& X=-\frac{d F}{d t}-Q \beta \frac{d \theta}{d z}, \\
& Y=-\frac{d G}{d t}+Q \alpha \frac{d \theta}{d z} ;
\end{aligned}
$$

where $\alpha$ and $\beta$ are the components of the magnetic force parallel to $x$ and $y$ respectively.

Hence if $K$ be the specific inductive capacity

$$
\left.\begin{array}{l}
\frac{4 \pi}{K} f=-\frac{d F}{d t}-Q \beta \frac{d \theta}{d z} \\
\frac{4 \pi}{K} g=-\frac{d G}{d t}+Q \alpha \frac{d \theta}{d z}
\end{array}\right\} .
$$

Differentiating the first of these equations with respect to $t$ we get

$$
\frac{4 \pi}{K} \frac{d f}{d t}=-\frac{d^{2} F}{d t^{2}}-Q \frac{d \beta}{d t} \frac{d \theta}{d z}
$$

but in a dielectric

$$
4 \pi \mu \frac{d f}{d t}=-\frac{d^{2} F}{d z^{2}},
$$


and in the small term

$$
Q \frac{d \beta}{d t} \frac{d \theta}{d z}
$$

if we neglect $Q^{2}$ we may put

$$
\mu \frac{d \beta}{d t}=\frac{d^{2} F}{d t d z}
$$

where $\mu$ is the magnetic permeability of the dielectric.

Substituting these values in equation (137) we get

$$
\frac{\mathrm{I}}{\mu K} \frac{d^{2} F}{d z^{2}}=\frac{d^{2} F}{d t^{2}}+\frac{Q}{\mu} \frac{d \theta}{d z} \frac{d^{2} F}{d t d z} \ldots \ldots \ldots(\mathbf{1} 38) .
$$

For a circularly polarized ray we may put

$$
\left.\begin{array}{r}
F=A \sin \frac{2 \pi}{\lambda}(v t-z) \\
G=-A \cos \frac{2 \pi}{\lambda}(v t-z)
\end{array}\right\}
$$

where $V$ is the velocity of the light, and $\lambda$ its wave length.

Substituting this value for $F$ in equation (1 38 ) we get

$$
\frac{\mathrm{I}}{\mu K}=v^{2}-\frac{Q}{\mu} \frac{d \theta}{d z} v,
$$

and thus the velocity of the ray is greater than if the temperature had-been uniform by

$$
\frac{\mathrm{I}}{2} \frac{Q}{\mu} \frac{d \theta}{d z} \text {. }
$$

The velocity of propagation of a ray circularly polarized in the opposite sense will also be increased by the same amount. So that regarding a plane polarized ray as made up of two rays circularly polarized in opposite senses we see that when such a ray passes through a medium in which there is a steady flow of heat, the plane of rotation will not be rotated, but the velocity will be increased by

$$
\frac{1}{2} \frac{Q}{\mu} \frac{d \theta}{d z} \text {. }
$$


Thus even if we had a transparent substance for which $Q$ was as great as for bismuth, viz. 13 and a fall of temperature of $100^{\circ} \mathrm{C}$. per millimetre, the change in the velocity would only amount to

$$
\frac{1}{2} \times 13 \times 10^{3}
$$

or

65 ,

this change is only about $2 \cdot 2 \times 10^{-7}$ per cent. of the velocity of light, and violet light would have to traverse about $20,000 \mathrm{c.m}$. to gain or lose a wave length. This effect therefore is much too small to be detected experimentally.

We saw by equation (r35) that when electric displacements take place in a field in which the temperature is not uniform, heat is absorbed or evolved, so that we should expect thermal changes to accompany the propagation of a ray of light through a medium the temperature of which was not uniform.

By equation (I35) the heat $\delta H$ which must be supplied in unit time to unit volume of the medium to prevent the temperature changing, is if the heat is flowing along the axis of $z$, given by the equation

$$
\delta H=Q \frac{d \theta}{d z}\{a \dot{g}-\beta \dot{f}\} .
$$

Let us take the case of a plane polarized ray for which approximately

$$
\begin{aligned}
& f=A \cos \frac{2 \pi}{\lambda}(v t-z) \\
& g=0 \\
& a=0 \\
& \beta=4 \pi v A \cos \frac{2 \pi}{\lambda}(v t-z),
\end{aligned}
$$


thus

$$
\delta H=Q \frac{8 \pi^{2} v^{2} \cdot A^{2}}{\lambda} \frac{d \theta}{d z} \cos \frac{2 \pi}{\lambda}(z t-z) \sin \frac{2 \pi}{\lambda}(v t-z) .
$$

So that the propagation of light along an unequally heated medium would if this theory is correct be accompanied by periodic emission and absorption of heat, analogous to that which accompanies the propagation of soundaccording to Laplace's theory. According to Maxwell (Electricity and Magnetism, Vol. II. p. 402) the maximum value of $\beta$ for strong sunlight is 193 so that

and therefore

$$
4 \pi v A=\cdot 193
$$

$$
8 \pi^{2} 7^{2} A^{2}=\cdot 02,
$$

let us take $\lambda$ as $3.9 \times 10^{-5}$ the wave length of the violet ray $H$ in air

$$
\delta H=5 \times 10^{2} \times Q \times \frac{d \theta}{d z} \cos \frac{2 \pi}{\lambda}(v t-z) \sin \frac{2 \pi}{\lambda}(v t-z) .
$$

If $Q$ were as large as it is for bismuth, i.e. $13^{2}$, and there was a fall of $100^{\circ} \mathrm{C}$. - in one centimetre, then the maximum amount of heat absorbed or emitted would be

$$
3.3 \times 10^{3}
$$

this would correspond to changes of temperature of not more than one ten thousandth of a degree centigrade, if the specific heat of the substance were as great as that of water.

62. Longitudinal effect. v. Ettinghausen and Ernst found that in addition to the transversal electromotive force there was a longitudinal one along the lines of flow of the heat, which was not reversed when the magnetizing force was reversed, and which was proportional to the square of the magnetizing force as long as this was small. This 
shows that the quantity $\sigma$ which we considered when we were discussing the Thomson effect in $\S 5 \mathrm{I}$ is a function of the square of the magnetic force. If we consider the effect of this term magnetically we shall see that it indicates that the magnetic permeability of a magnet will be affected by the proximity of a conductor throughout which electricity is distributed.

63. It is interesting, because suggestive of new physical phenomena, to trace the consequence of the existence in the Lagrangian function of terms, which are symmetrical functions of $f, g, h, \alpha, \beta, \gamma$ and their differential coefficients, such as terms proportional to

$$
\begin{gathered}
f \alpha+g \beta+h \gamma \\
f \frac{d \alpha}{d x}+g \frac{d \beta}{d y}+h \frac{d \gamma}{d z}, \\
\alpha \frac{d f}{d x}+\beta \frac{d g}{d y}+\gamma \frac{d h}{d z}, \\
f\left(\frac{d \beta}{d z}-\frac{d \gamma}{d y}\right)+g\left(\frac{d \gamma}{d x}-\frac{d \alpha}{d z}\right)+h\left(\frac{d \alpha}{d y}-\frac{d \beta}{d x}\right), \\
\left(\frac{d \beta}{d z}-\frac{d \gamma}{d y}\right)\left(\frac{d g}{d z}-\frac{d h}{d y}\right)+\left(\frac{d \gamma}{d x}-\frac{d \alpha}{d z}\right)\left(\frac{d h}{d x}-\frac{d f}{d z}\right) \\
+\left(\frac{d \alpha}{d y}-\frac{d \beta}{d x}\right)\left(\frac{d f}{d y}-\frac{d g}{d x}\right) .
\end{gathered}
$$

The reader however who is interested in this will have no difficulty in tracing the consequences of these terms by the methods already given. 


\section{CHAPTER VIII.}

ON “RESIDUAL” EFFECTS.

64. THERE are a great many cases in which the application of forces to a body seems to produce a change in it, from which it does not recover for some time after the forces have been removed.

Thus, for example, if we keep a metal wire or glass fibre twisted for some time, it will not when the twisting couple is removed at once vibrate symmetrically about its original position of equilibrium, but will oscillate about a new zero which gradually approaches the old one, the maximum difference between the temporary and the true zero and the time which elapses before these coincide increasing within certain limits with the duration of the original twisting couple.

Phenomena of this kind are called in German treatises "elastiche nachwirkung." This peculiar effect of torsion does not seem to have received a name in this country, but the analogous cases in electricity and magnetism are called respectively "residual charge" and "residual magnetism." This latter effect is only partly analogous to that of the twisted wire, as they differ in one very important respect, that of permanence. In the case of the twisted 
wire the effect of the previous torsion will disappear if time be given to it, but soft iron if kept free from disturbance seems to be able to retain its magnetism for any length of time.

We shall now endeavour to find a dynamical analogue to the case of the twisted wire. Let us suppose that we have a frictionless machine whose configuration is fixed by one coordinate $x$ and that this is connected with another machine fixed by the coordinate $y$, the motion of this machine being resisted by a frictional force proportional to the velocity. We shall suppose at first that the mass of the second machine is so small that its inertia may be neglected, and that the connexion between the two machines is expressed by the existence in their Lagrangian function of a term $f(x y)$ which involves both $x$ and $y$, but not their differential coefficients with respect to the time. Then if the force $X$ acting on the first machine is the only external force acting on the system, the equations of motion will be of the form

$$
\begin{aligned}
A \frac{d^{2} x}{d t^{2}}+\mu x-\frac{d}{d x} f(x y) & =X \ldots \ldots \ldots(\mathrm{r} 40), \\
b \frac{d y}{d t}+a y-\frac{d}{d y} f(x y) & =0 \ldots \ldots \ldots(\mathrm{1} 4 \mathrm{r}) .
\end{aligned}
$$

If $x$ and $y$ are small we may put

$$
\begin{aligned}
& \frac{d}{d x} f(x y)=\alpha x+\beta y, \\
& \frac{d}{d y} f(x y)=\beta x+\gamma y,
\end{aligned}
$$

where $\alpha, \beta, \gamma$ are constants.

Making these substitutions we have

T. D. 


$$
\begin{gathered}
A \frac{d^{2} x}{d t^{2}}+(\mu-\alpha) x-\beta y=X \ldots \ldots \ldots(\mathrm{I} 42), \\
\quad b \frac{d y}{d t}+(a-\gamma) y-\beta x=0 \ldots \ldots \ldots \ldots(\mathrm{I} 43) .
\end{gathered}
$$

The solution of (143) is

$$
y=\frac{\beta}{b} \int_{0}^{t} \epsilon^{-\frac{(a-\gamma)}{b}(t-t)} x d t^{\prime} \ldots \ldots \ldots . .(\mathbf{I} 44),
$$

substituting this value of $y$ in equation $\left(\mathrm{r}_{42}\right)$ we get

$$
A \frac{d^{2} x}{d t}+(\mu-\alpha) x-\frac{\beta^{2}}{b} \int_{0}^{t} \epsilon^{-\frac{(a-\gamma)}{b}\left(t-t^{\prime}\right)} x d t^{\prime}=X \ldots \text { (145). }
$$

We see by this equation that the effect on the first system of its connexion with the second is to make the forces called into play at any time by the displacement of the system from its position of equilibrium depend not merely upon the displacement of the system at that time but also upon the previous displacements, and that a displacement $x$ lasting for a short time $\tau$ produces after a time $T$ a force $\tau x \psi(T)$ where

$$
\psi(T)=\frac{\beta^{2}}{b} \epsilon^{-\frac{(a-\gamma)}{b} T} .
$$

Neesen ("Elastiche Nachwirkung bei Torsion," Berlin Monatsberichte, Feb. I2, I874, p. I4I) has shown that the assumption that $\psi(T)$ is proportional to $\epsilon^{-k T}$ agrees with his experiments on the twisting of wires. Boltzmann (Sitz. der k. Akad. zu Wien, 70, p. 275, 1874) works out a theory where $\psi(T)$ is proportional to $\mathbf{I} / T$.

In many cases we are given the forces at the time $t^{\prime}$ and not the displacement, and in these, equation (I45) is not convenient. If as is generally the case the motion is so slow that we may neglect the effects of inertia, then we have 


$$
\begin{gathered}
b \frac{d y}{d t}+(a-\gamma) y=\beta x \\
(\mu-a) x=\beta y+X,
\end{gathered}
$$

so that

and therefore

$$
b \frac{d y}{d t}+\left\{(a-\gamma)-\frac{\beta^{2}}{\mu-a}\right\} y=\frac{\beta}{\mu-a} X,
$$

where

$$
y=\frac{\beta}{b(\mu-a)} \int_{0}^{t} \epsilon^{-k\left(t-t^{\prime}\right)} X d t^{\prime}
$$

$$
k=\frac{a-\gamma}{b}-\frac{\beta^{2}}{b(\mu-a)},
$$

and thus when the external force is removed

$$
x=\frac{\beta^{2}}{b(\mu-\alpha)^{2}} \int_{0}^{t} \epsilon^{-k\left(t-t^{\prime}\right)} X d t^{\prime} .
$$

If the primary machine had been connected with several secondaries instead of with only one, we should have, if the displacements are given

$$
x=\int_{0}^{t} x\left\{\Sigma \frac{\beta^{2}}{b(\mu-a)} \epsilon^{-\frac{(a-\gamma)}{b}\left(t-t^{\prime}\right)}\right\} d t^{\prime},
$$

and if the forces are given

$$
\begin{aligned}
x & =\int_{0}^{t} X \Sigma\left\{\frac{\beta^{2}}{b(\mu-\alpha)^{2}} \epsilon^{-k(t-t)}\right\} d t^{\prime} \\
& =\Sigma \epsilon^{-k t} \int_{0}^{t} \varpi X \epsilon^{k t^{\prime}} d t^{\prime} \ldots \ldots \ldots \ldots \ldots \ldots \ldots(\mathbf{I} 46),
\end{aligned}
$$

where $\varpi$ is written for $\beta^{2} / b(\mu-\alpha)^{2}$, and the sum taken for all the secondary systems. This is the general expression for the residual effect in terms of the forces acting on the system when it was under constraint.

If we cannot neglect the inertia of the secondary system we must introduce the term $B d^{2} y / d t^{2}$ into equation (143) so that that equation becomes 


$$
B \frac{d^{2} y}{d t^{2}}+b \frac{d y}{d t}+(a-\gamma) y=\beta x
$$

of which the solution corresponding to (I 44 ) is

$$
y=\frac{\mathrm{I}}{B\left(\lambda_{1}-\lambda_{2}\right)} \int_{0}^{t}\left\{\epsilon^{\lambda_{1}\left(t-t_{1}\right)}-\epsilon^{\lambda_{2}\left(t-t^{\prime}\right)}\right\} x d t^{\prime},
$$

where $\lambda_{1}$ and $\lambda_{2}$ are the roots of the equation

$$
B \lambda^{2}+b \lambda+a-\gamma=0 \text {. }
$$

Thus the introduction of inertia into the secondary system does not change the form of the solution, it only introduces fresh terms of the same type as those which previously existed, and the general solution is of the form

$$
\begin{aligned}
x & =\int_{0}^{t} x\left\{\Sigma_{C \epsilon}-\lambda\left(t-t^{\prime}\right)\right\} d t^{\prime} \\
& =\Sigma_{\epsilon}-\lambda t \int_{0}^{t} c x \epsilon^{\lambda t^{\prime}} d t^{\prime},
\end{aligned}
$$

where $c$ is a constant which depends on the constitution of the secondary system but not upon $x$. This is the general expression for the residual effect in terms of the initial displacements.

65. In those cases in which residual effects occur we may suppose that the secondary systems which are affected by the changes in the primary are the molecules of the body which is the seat of the phenomenon or a portion of such molecules. For example in the case of the residual charge of the Leyden jar we may look upon the electrical system as the primary system and the system consisting of the molecules of the glass as the secondary system, and may suppose that during the actions of the electromotive force on the glass, the arrangement of the molecules of the glass suffers gradual changes which react upon the electric displacement. 
The following extract from Clerk-Maxwell's article on the "Constitution of Bodies" in the Encyclopadia Britannica. is most instructive on this point.

"We know that the molecules of all bodies are in motion. In gases and liquids the motion is such that there is nothing to prevent any molecule from passing from any part of the mass to any other part; but in solids we must suppose that some, at least, of the molecules merely oscillate about a certain mean position, so that if we consider a certain group of molecules, its configuration is never very different from a certain stable configuration about which it oscillates.

"This will be the case even when the solid is in a state of strain provided the amplitude of the oscillations does not exceed a certain limit, but if it exceeds this limit the group does not tend to return to its former configuration but begins to oscillate about a new configuration of stability, the strain in which is either zero or at least less than in the: original configuration.

"The condition of this breaking up of a configuration. must depend partly on the amplitude of the oscillations and partly on the amount of strain in the original configuration; and we may suppose that different groups of molecules, even in a homogeneous solid, are not in similar circumstances in this respect.

"Thus we may suppose that in a certain number of groups the ordinary agitation of the molecules is liable to accumulate so much that every now and then the configuration of one of the groups breaks up, and this whether it is in a state of strain or not. We may in this case assume that in every second a certain proportion of these groups break up and assume configurations corresponding to a strain uniform in all directions. 
"If all the groups were of this kind, the medium would be a viscous fluid.

"But we may suppose that there are other groups, the configuration of which is so stable that they will not break up under the ordinary agitation of the molecules unless the average strain exceeds a certain limit, and this limit may be different for different systems of these groups.

"Now if such groups of greater stability are disseminated through the substance in such abundance as to build up a solid framework, the substance will be a solid which will not be permanently deformed except by a stress greater than a certain given stress.

"But if the solid also contains groups of smaller stability and also groups of the first kind which break up of themselves, then when a strain is applied the resistance to it will gradually diminish as the groups of the first kind break up, and this will go on till the stress is reduced to that due to the more permanent groups. If the body is now left to itself, it will not at once return to its original form but will only do so when the groups of the first kind have broken up so often as to get back to their original state of strain.

"This view of the constitution of a solid, as consisting of groups of molecules some of which are in different circumstances from others, also helps to explain the state of the solid after a permanent deformation has been given to it. In this case some of the less stable groups have broken up and assumed new configurations, but it is possible that others more stable may retain their original configurations, so that the form of the body is determined by the equilibrium between these two sets of groups; but if on account of rise of temperature, increase of moisture, violent vibration, or any other cause, the breaking up of the less stable groups is facilitated, the more stable groups may again assert their sway 
and tend to restore the body to the shape it had before its deformation."

66. Let us now apply equation (146) to a definite case. Let us suppose that the force $X_{1}$ acts on the system from $t=0$ to $i=T_{1}$ and that from $t=T_{1}$ to $t=T_{2}$, the force $-X_{2}$ acts, then we have by equation ( 146 )

$$
\begin{aligned}
x & =\varpi \int_{0}^{t} \epsilon^{-k\left(t-t^{\prime}\right)} X d t^{\prime} \\
& =\varpi \epsilon^{-k t}\left\{\int_{0}^{T_{1}} \epsilon^{k t^{\prime}} X_{1} d t^{\prime}-\int_{T_{1}}^{T_{2}} \epsilon^{k t^{\prime}} X_{2} d t^{\prime}\right\} \\
& =\epsilon^{-k t} \frac{\varpi}{k}\left\{X_{1}\left(\epsilon^{k T_{1}}-\mathrm{I}\right)-X_{2}\left(\epsilon^{k T_{2}}-\epsilon^{k T_{1}}\right)\right\} \ldots(\mathrm{I} 47) .
\end{aligned}
$$

If the primary system is connected with several secondaries instead of one then we have

$$
\begin{aligned}
x=X_{1} \Sigma \frac{\varpi}{k}\left(\epsilon^{-k\left(t-T_{1}\right)}-\epsilon^{-k t}\right) & \\
& -X_{2} \Sigma \frac{\varpi}{k}\left\{\epsilon^{-k\left(t-T_{2}\right)}-\epsilon^{-k\left(t-T_{1}\right)}\right\} \ldots(\mathbf{I} 48) .
\end{aligned}
$$

We see from equation (I47) that if we have only one secondary $x$ will never change sign, but that the system will return slowly to its position of equilibrium and never get beyond it whatever may have been its previous history. We know however that in the case of residual torsion of glass fibres and the residual charge of a Leyden jar the residual effects may be made to change sign. Thus if we give a fibre a strong twist in the positive direction for some considerable time and then a twist in the negative direction for a short time, the residual torsion after the twisting couple is taken off may be first in the negative and then in the positive direction. This is sometimes expressed by saying that the residual charges come out in the inverse order to that in which they went in. 
If there are only two values of $k, k_{1}$ and $k_{2}$, then since

$$
\left.x=\epsilon^{-k_{1} t} \int_{0}^{t} \varpi X \epsilon^{k_{1}} t^{\prime} d t^{\prime}+\epsilon^{-k_{2} t} \int_{0}^{t} \varpi X \epsilon^{k_{2} t^{\prime}} d t \ldots \ldots \text { (1 } 49\right)
$$

and since after the external force is removed

$$
\int_{0}^{t} \varpi X \epsilon^{k t^{\prime}} d t^{\prime}
$$

is not a function of $t$, we see that the sign of the residual effect can only change once however complicated the alternations in the signs of the twists or electrifications previously applied to the system may have been.

Dr John Hopkinson represented the residual charge of a Leyden jar by a formula of the same type as ( $\mathrm{r} 49$ ) (see Chrystal's art. Electricity, Encyclopadia Britannica, p. 40) and he showed that for the formula to agree with his experiments on the residual charge in glass it was necessary to take more than two values of $k$. Now when we included the effects of the inertia of the secondary system we got two, but only two values of $k$ for each secondary, so that as we have to introduce more terms than two to represent the residual effect in glass we must have more than one secondary system. This is an indication that glass is not a homogeneous substance but a mixture of different silicates.

According to Neesen (loc. cit.) the residual effects of torsion in silk and guttapercha fibres can be represented by a single term of the form $c \epsilon^{-k t}$.

67. We shall now investigate another effect due to the same cause as the residual effect but of a different kind. This is the effect of the secondary system on the way in which the free vibrations of the primary die away.

Using the same notation as before and neglecting the inertia of the secondary system we have for the free vibrations the equation 
RESIDUAI, EFFECTS.

$$
\begin{gathered}
A \frac{d^{2} x}{d t^{2}}+(\mu-\alpha) x=\beta y \\
b \frac{d y}{d t}+(a-\gamma) y=\beta x, \\
A \frac{d^{2} x}{d t^{2}}+\mu^{\prime} x=\beta y \\
b \frac{d y}{d t}+a^{\prime} y=\beta x
\end{gathered}
$$

eliminating $y$ we have

$$
\left(b \frac{d}{d t}+a^{\prime}\right)\left(A \frac{d^{2}}{d t^{2}}+\mu^{\prime}\right) x=\beta^{2} x \ldots \ldots \ldots(150) .
$$

As this is a linear equation let us assume that $x$ varies as $\epsilon^{p t}$, then $p$ is given by the equation

$$
\begin{gathered}
\left(b p+a^{\prime}\right)\left(A p^{2}+\mu^{\prime}\right)=\beta^{2}, \\
A p^{2}+\mu^{\prime}=\frac{\beta^{2}}{b p+a^{\prime}} .
\end{gathered}
$$

or

The right hand side of this equation is small, and if the residual effect does not produce a large change in the period of vibration we may on the right hand side of the equation substitute for $p$ its value when there is no secondary system, i.e. $i\{\mu / A\}^{\frac{1}{2}}$ and for $a^{\prime}, a$; making these substitutions we have

$$
A p^{2}+\mu^{\prime}=\frac{\beta^{2}}{a^{\prime}+b i(\mu / A)^{\frac{1}{2}}}=\frac{\beta^{2}\left\{a-i b(\mu / A)^{\frac{1}{2}}\right\}}{a^{2}+b^{2} \mu / A},
$$

$$
\begin{gathered}
\text { or } \quad p^{2}=-\frac{\mu^{\prime}}{A}+\frac{\beta^{2}}{A} \frac{\left\{a-i b(\mu / A)^{\frac{1}{2}}\right\}}{a^{2}+b^{2} \mu / A}, \\
\text { or } \quad p=i\left\{\frac{\mu^{\prime}}{A}\right\}^{\frac{1}{2}}\left[\mathrm{I}-\frac{\beta^{2}}{\mu^{\prime}} \frac{\left\{a-i b(\mu / A)^{\frac{1}{2}}\right\}}{a^{2}+b^{2} \mu / A}\right]^{\frac{1}{2}} .
\end{gathered}
$$

So that if $\beta^{2}$ be small

$$
p=i\left\{\frac{\mu^{\prime}}{A}\right\}^{\frac{1}{2}}\left[\mathrm{I}-\frac{1}{2} \frac{\beta^{2}}{\mu^{\prime}} \frac{\left\{a-i b(\mu / A)^{\frac{1}{2}}\right\}}{a^{2}+b^{2} \mu / A}\right] \text { approximately. }
$$


And thus the real part of $p$ is approximately

$$
-\frac{1}{2} \frac{b \beta^{2}}{A a^{2}+\mu b^{2}}
$$

the amplitude of the vibrations of $x$ are thus given by the expression

$$
\exp \left(-\frac{1}{2} \frac{b \beta^{2}}{A a^{2}+\mu b^{2}} t\right)
$$

where $\exp (x) \equiv e^{x}$.

So that the ratio of the amplitudes of two successive swings is

$$
\exp \left(-\frac{1}{2} \frac{b \beta^{2}}{A a^{2}+\mu b^{2}} T\right) \ldots \ldots \ldots \ldots\left(\mathrm{r}_{5} \mathrm{r}\right),
$$

where $T$ is the time of a complete oscillation, and is given by the equation,

$$
T=2 \pi\{A / \mu\}^{\frac{1}{2}} \text { approximately. }
$$

Substituting this value of $T$ in $\left(\mathrm{r}_{5} \mathrm{r}\right)$ we get for the ratio of the two amplitudes the expression

$$
\exp \left(-\frac{\pi b \beta^{2} A^{\frac{1}{2}} \mu^{-\frac{1}{2}}}{A a^{2}+\mu b^{2}}\right) \text {. }
$$

Now if the motion of $x$ were resisted by a frictional force proportional to the velocity, the equation for $x$ would be

$$
A \frac{d^{2} x}{d t^{2}}+\lambda \frac{d x}{d t}+\mu x=0,
$$

the solution of which is

$$
x=C e^{-\frac{\lambda t}{2 A}} \cos \left\{\left(\frac{\mu}{A}-\frac{\lambda^{2}}{4 A^{2}}\right)^{\frac{1}{2}} t+\epsilon\right\},
$$

where $C$ and $\epsilon$ are constants.

The ratio of the amplitudes of two successive swings in this case is

$$
\exp \left(-\frac{\lambda T}{2 A}\right)
$$


or approximately

$$
\exp \left(-\frac{\lambda}{A} \pi \frac{A^{\frac{1}{2}}}{\mu^{\frac{1}{2}}}\right)
$$

When the decrease in the amplitude is due to the connexion with the secondary system, the ratio of two successive amplitudes is

$$
\exp \left(-\frac{\pi b^{2} \beta A^{\frac{1}{2}} \mu^{-\frac{1}{2}}}{A a^{2}+\mu b^{2}}\right),
$$

so that the logarithmic decrement when the resistance is frictional varies as

$$
\frac{\mathrm{I}}{A^{\frac{1}{2}} \mu^{\frac{1}{2}}}
$$

when it is due to the secondary system it varies as

$$
\frac{\mathrm{I}}{A^{\frac{1}{2}}\left\{\mathrm{I}+\frac{\mu b^{2}}{A a^{2}}\right\} \mu^{\frac{1}{2}}} .
$$

Hence we see that if the mass of the vibrating body is altered, the variation of the logarithmic decrement will be less in this case than it would if the decay in the oscillations were due to friction. This agrees with the results of Sir William Thomson's experiments on the decay of the torsional vibrations of wire, as he found that the loss was greater with the longer periods than that calculated according to the law of square roots from its amount in the experiment with shorter periods. In fact if $A$ were much smaller than $\mu b^{2} / a^{2}$ the rate of decay would be increased instead of diminished by increasing the vibrating mass, as the rate of decay has its maximum value when $A=\mu b^{2} / a^{2}$. 


\section{CHAPTER IX.}

INTRODUCTORY TO THE STUDY OF REVERSIBLE SCALAR PHENOMENA.

68. So far we have been dealing with phenomena in which as in ordinary dynamics the quantities concerned were mainly of a vector character. We shall now however go on to consider phenomena when the quantities we have to deal with are chiefly scalar, such as the phenomena of evaporation, dissociation, chemical combination, etc. where the quantities which have to be considered are such things as temperature, vapour density, or the number of molecules in a particular state. The chief difference between these cases and those we have been considering is that in these we have as in the kinetic theory of gases to deal chiefly with the average values of certain quantities and cannot attempt to follow the variations of the individual members which make up the average, while in the previous cases we have been able to follow in all detail the changes in most of the quantities introduced. In these new cases all that we can get by the application of the Hamiltonian principle are relations between the averages of a series of quantities; as however these averages are all that we can 
observe in these cases, this limitation is not serious from a practical point of view.

The relations we shall deduce are those which exist when the body is in a steady state.

69. The systems we shall have to consider are portions of matter in the solid, liquid or gaseous state, and consist, according to the molecular theory of bodies, of a very large number of secondary systems or molecules. Now we can control a primary system in many ways, we can fix its geometrical position, we can within certain limits strain it in any way we please, we may establish electric currents or electric displacements through it, and if the body is magnetic we can magnetize it within the limits of saturation: so that the coordinates fixing the geometrical, the strain, the electric and the magnetic configurations are under our control and have therefore been called $(\S 46)$ controllable coordinates.

The coordinates fixing the positions of the several secondary systems are not however within our control and we have not the power of altering any one of them; we have called these unconstrainable coordinates.

70. When we say that a system consisting of a great number of molecules is in a steady state we mean that the state is steady with respect to the controllable coordinates and make no supposition as to whether it is so or not with respect to the unconstrainable ones, all that we shall assume is that the mean values which we can observe and which depend upon the unconstrainable coordinates are steady.

Thus when the system is in a steady state the velocity of each controllable coordinate must be constant, and if the coordinate enters explicitly into the expression for either the kinetic or potential energy, that is if the coordinate is not a "kinosthenic" or speed one, the velocity must vanish. 
71. We shall now proceed to prove that when a system consisting of a great number of molecules is in a steady state the mean value of the Lagrangian function has a stationary value so long as the velocities of the controllable coordinates are not altered.

Let us denote the controllable speed coordinates by the symbol $q_{1}$, the controllable positional coordinates by $q_{2}$ and the unconstrainable coordinates by $q_{3}$, then we have by the Calculus of Variation

$$
\begin{gathered}
\delta \int_{t_{0}}^{t_{1}} L d t=\Sigma\left(\frac{d L}{d \dot{q}_{2}} \delta q_{2}\right)_{t_{0}}^{t_{1}}+\mathbf{\Sigma}\left(\frac{d L}{d \dot{q}_{3}} \delta q_{3}\right)_{t_{0}}^{t_{1}}+\Sigma \int_{t_{0}}^{t_{1}} \frac{d L}{d \dot{q}_{1}} \delta \dot{q}_{1} d t \\
\quad+\Sigma \int_{t_{0}}^{t_{1}}\left(\frac{d L}{d q_{2}}-\frac{d}{d t} \frac{d L}{d \dot{q}_{2}}\right) \delta q_{2} d t+\Sigma \int_{t_{0}}^{t_{1}}\left(\frac{d L}{d q_{3}}-\frac{d}{d t} \frac{d L}{d \dot{q}_{3}}\right) \delta q_{3} d t .
\end{gathered}
$$

Remembering Lagrange's Equations we see that this equation reduces to

$$
\delta L=\Sigma \int_{t_{0}}^{t_{1}} \frac{d L}{d \dot{q}_{1}} \delta \dot{q}_{1} d t+\Sigma\left(\frac{d L}{d \dot{q}_{2}} \delta q_{2}\right)_{t_{0}}^{t_{1}}+\Sigma\left(\frac{d L}{d \dot{q}_{3}} \delta q_{3}\right)_{t_{0}}^{t_{1}} \ldots\left(\mathrm{I} 5^{2}\right) .
$$

Let us suppose that the symbol of variation refers to a disturbed motion in which the values of the controllable coordinates are slightly altered while the velocities of the speed coordinates remain unaltered and constant during the disturbed as well as the undisturbed motion.

We shall for the sake of greater clearness consider the three terms on the right-hand side of equation (152) separately, as the considerations which apply to them are different in each case.

72. Let us first take the term

$$
\Sigma \int_{t_{0}}^{t_{1}} \frac{d L}{d \dot{q}_{1}} \delta \dot{q}_{1} d t
$$


Since we suppose that in the disturbed motion the velocities of the speed coordinates are unaltered $\delta \dot{q}$, is always zero, and thus the term we are considering vanishes.

73. We shall now show that the term

$$
\left[\mathbf{\Sigma} \frac{d L}{d \dot{q}_{2}} \delta q_{2}\right]_{t_{0}}^{t_{1}}
$$

also vanishes. Since $q_{2}$ is not a speed coordinate it must enter explicitly into the expression $L$, so that when the motion is steady the velocities of coordinates of this class vanish. The terms in $L$ which contain the velocities of positional coordinates always vanish when the motion is steady. They do not therefore contribute anything to the mean value of $L$, and so we may without loss of generality suppose that the coefficients of terms in the kinetic energy involving the velocities of positional coordinates are all zero and that therefore $\frac{d L}{d \dot{q}_{2}}$ may be put equal to zero. In this way we may see that the terms we are considering in the expression for the variation of the mean value of the Lagrangian function vanish.

74. To show that the terms

$$
\left[\frac{d L}{d \dot{q}_{3}} \delta q_{3}\right]_{t_{0}}^{t_{1}}
$$

vanish we must use the reasoning given by Clausius in his paper "On the Second Axiom of the Mechanical Theory of Heat," Phil. Mag. xuir. p. I78.

Let us in the first place consider what the coordinates denoted by $q_{3}$ are. They are coordinates fixing the position of the molecules of the system and may be 
divided into two classes: firstly, coordinates fixing the position of the centres of mass of the molecules, and secondly, coordinates fixing the position of the molecules relatively to their centres of mass. The motion of the latter coordinates will be periodic, while that of the former will not be so; in consequence however of the frequent collisions between the molecules their direction of motion will be continually reversed, so that if the position of a molecule be arbitrarily changed the distance between the disturbed and the undisturbed positions will not increase indefinitely with the time, the difference will sometimes be positive, some. times negative, but will fluctuate between limits which do not increase with the time. Thus if $q_{3}^{\prime}$ is a coordinate of this kind

$$
\left[\frac{d L}{d \dot{q}^{\prime}} \delta q^{\prime}\right]_{t_{0}}^{t_{1}}
$$

will fluctuate between positive and negative values which do not increase with the interval $t_{0}-t_{1}$.

The same reasoning will apply with still greater force to those coordinates which fix the configuration of a molecule relatively to its centre of mass, for these coordinates will oscillate and therefore the part of

$$
\left[\frac{d L}{d \dot{q}_{3}} \delta q_{3}\right]_{t_{0}}^{t_{1}}
$$

depending upon these coordinates will fluctuate between limits which do not increase as the time $t_{1}-t_{0}$ increases.

Now any change which we have the power to produce in any of the coordinates fixing the system will, since the motion is steady, produce a change in each term of

$$
\int_{t_{0}}^{t_{1}} L d t
$$


which will increase proportionately to the increase in the interval $t_{1}-t_{0}$ : and thus if we integrate over a sufficiently long interval we may neglect any terms on the right hand side of equation $\left(15^{2}\right)$ which fluctuate between fixed values and therefore as far as coordinates of the kind $q_{3}$ are concerneci put

$$
\delta \int_{t_{0}}^{t_{1}} L d t=0
$$

when the interval $t_{1}-t_{0}$ is sufficiently long.

We have seen however that this is also true as far as the variations of the other coordinates $q_{1}, q_{2}$ are concerned, so that when the motion is steady we have

$$
\delta(\bar{L})=0 \ldots \ldots \ldots \ldots \ldots \ldots \ldots\left(\mathbf{r}_{53}\right),
$$

where $\bar{L}$ denotes the mean value of $L$ taken over unit time, e.g. one second, and where the variations are such as could be produced by slightly altering the values of the coordinates. We may conclude that one second is a sufficiently long interval over which to integrate since according to the molecular theory of gases there are both a great many collisions and a great many vibrations in this period.

75. In the above investigation we have supposed that the Lagrangian function $L$ is expressed in terms of the velocities of the coordinates and the proof is only valid when it is so expressed and does not hold when the velocities corresponding to some coordinates are eliminated and the momenta corresponding to them introduced instead.

We can prove however in this case that the modified Lagrangian function $\left(\S_{\mathrm{II}}\right)$ is stationary when the system is in a state of steady motion.

For let $L^{\prime}$ be the modified Lagrangian function and $q$ a T. D. 
coordinate whose velocity has not been eliminated, then $L^{\prime}$ is a function of $q, \dot{q} \ldots$ and the momenta corresponding to the other coordinates, and since

$$
\frac{d}{d t} \frac{d L^{\prime}}{d \dot{q}}-\frac{d L^{\prime}}{d q}=0
$$

we have by the Calculus of Variations

$$
\delta \int_{t_{0}}^{t_{1}} L^{\prime} d t=\left\{\Sigma \frac{d L^{\prime}}{d q} \delta q\right\}_{t_{0}}^{t_{1}}+\mathrm{\Sigma} \int_{t_{0}}^{t_{1}} d L^{\prime} \delta p d t \ldots \text { ( ( 54), }
$$

where $p$ is the momentum corresponding to one of the eliminated coordinates. We can prove exactly as before that the right hand side of equation (154) vanishes for all variations in which the momenta corresponding to the eliminated coordinates remain unaltered.

Thus we have in all cases an equation of the form

$$
\delta \bar{L}=\text { o, }
$$

where $\bar{L}$ is the mean value of the ordinary Lagrangian function or its modified form according as it does not or does contain the momenta corresponding to some of the s.oordinates.

76. In the physical applications of this principle it would sometimes be difficult to tell whether a symbol occurring in $L$ represented a momentum or a velocity. Fortunately however this knowledge is unnecessary if we calculate the Lagrangian function from the forces required to preserve equilibrium. For when the system is in a steady state, $X$ the force of type $x$ which must be applied to maintain equilibrium is given by

$$
X=-\frac{d L}{d x}
$$

where $L$ is the Lagrangian function or its modified form according as the kinetic energy does not or does contain the momenta corresponding to some of the coordinates. So 
that by what we have just proved

$$
-\Sigma \iint X d x d t . . . \ldots \ldots \ldots \ldots . . . .(\mathbf{r} 55),
$$

the sum being taken for all the coordinates and $X$ expressed in terms of them, is the expression for the terms depending on the controllable coordinates in a function which possesses the property of having a stationary value when the system to which it refers is in a șteady state.

77. Thus to take an example let us consider the case of a heavy particle whose mass is $m$ attached to a fixed point by a string whose length is $l$, and moving so that the string makes a constant angle $\approx$ with the vertical. The kinetic energy of the system is

$$
\frac{1}{2} m l^{2} \sin ^{2} \vartheta \dot{\phi}^{2}
$$

where $\phi$ is the angle which the plane containing the string and a vertical line makes with some fixed plane. The couple $\Theta$ which must act on the system to keep $\vartheta$ constant is

$$
-m{ }^{2} \sin \vartheta \cos \vartheta \dot{\phi}^{2} \text {. }
$$

When the system is acted on by gravity the potential energy is - $m g l \cos \vartheta$ so that the Lagrangian function is

$$
\frac{1}{2} m l^{2} \sin ^{2} \vartheta \dot{\phi}^{2}+m g l \cos \vartheta
$$

which may be written

$$
-\int \Theta d \theta+m g l \cos \vartheta
$$

and this possesses the property of being stationary.

If however the Lagrangian function is expressed in terms of $\Phi$ the momentum corresponding to $\phi$ and given by the equation

$$
\Phi=m l^{2} \sin ^{2} \vartheta \dot{\phi}
$$

the Lagrangian function becomes

$$
\frac{\mathrm{I}}{2 m l^{2}} \Phi^{2} \operatorname{cosec}^{2} \vartheta+m g l \cos \vartheta
$$

and this expression is not stationary. 
The function which possesses this property is the "modified" Lagrangian function

$$
-\frac{r}{2 m l^{2}} \Phi^{2} \operatorname{cosec}^{2} \vartheta+m g l \cos \vartheta \text {. }
$$

Since $\Theta$ however when expressed in terms of $\theta$ and $\Phi$ equals

$$
-\frac{\mathbf{I}}{m l^{2}} \Phi^{2} \frac{\cos \vartheta}{\sin ^{2} \vartheta}
$$

we see that the "modified" Lagrangian function again equals

$$
-\int \Theta d \theta+m g l \cos \vartheta
$$

Thus the expression

$$
-\int \Theta d \theta+m g l \cos \vartheta
$$

is stationary however $\Theta$ may be expressed, whether in terms of $\dot{\phi}$ or $\Phi$.

This example illustrates the principle that if we calculate the Lagrangian function from the forces necessary to preserve equilibrium we need not consider whether it is expressed in terms of velocities or momenta.

78. If we consider the proof by which the equation

$$
\delta \int_{t_{0}}^{t_{1}} L d t=\Sigma\left(\frac{d L}{d \dot{q}} \delta q\right)_{t_{0}}^{t_{1}} \ldots \ldots \ldots \ldots(\mathrm{I} 56)
$$

was established we shall notice one point which we must continually bear in mind when we are calculating the value of the potential energy.

By the Calculus of Variations

$$
\delta \int_{t_{0}}^{t_{1}} L d t=\int_{t_{0}}^{t_{1}}\left\{\frac{d L}{d q}-\frac{d}{d t} \frac{d L}{d \dot{q}}\right\} \delta q d t+\Sigma\left(\frac{d L}{d \dot{q}} \delta q\right)_{t_{0}}^{t_{1}},
$$


and thus if equation $\left(15^{6}\right)$ holds we must have

$$
\frac{d L}{d q}-\frac{d}{d t} \frac{d L}{d q}=0 \text {. }
$$

Now in ordinary Rigid Dynamics perhaps the most usual form of Lagrange's equation is

$$
\frac{d}{d t} \frac{d L}{d \dot{q}}-\frac{d L}{d q}=Q
$$

where $Q$ is the external force of type $q$ tending to increase this coordinate. In this case $L=T-V^{\prime}$ where $V^{\prime}$ is the potential energy when the coordinates have their assigned value and the system is free from the action of external forces. If however we are to use equation (I53) we must put $L=T-V$ where

$$
V=V^{\prime}-\Sigma \int Q d q
$$

that is we must add to the potential energy we are considering the potential energy of the system which produces the external forces.

Lagrange's equation may now be. written

$$
\frac{d}{d t} \frac{d T}{d \dot{q}}-\frac{d}{d q}(T-V)=0
$$

and equation ( 153 ) is true.

Thus to take an example, in Electrostatics we often assume that the potential energy $V^{\prime}$ of unit volume of a dielectric whose specific inductive capacity is $K$ and through which the electric displacements parallel to the axes of $x, y$; $z$ are respectively $f, g, h$ is

$$
\frac{4 \pi}{K}\left\{f^{2}+g^{2}+h^{2}\right\}
$$

and that the equations of equilibrium are

$$
\frac{d V^{\prime}}{d f}=X, \quad \frac{d V^{\prime}}{d g}=Y, \quad \frac{d V^{\prime}}{d h}=Z,
$$


where $X, Y, Z$ are the components of the electromotive force parallel to the axes of $x, y, z$ respectively.

If however we wish to apply the theorem we are now considering we must put

$$
V=\frac{4 \pi}{K}\left\{f^{2}+g^{2}+h^{2}\right\}-\left\{X f+Y_{\delta}+Z h\right\},
$$

for then the equations of equilibrium are

$$
\frac{d V}{d f}=\frac{d V}{d y}=\frac{d V}{d h}=0 \text {. }
$$

The necessity of choosing $V$ so that the equations of motion are of the form

$$
\frac{d}{d t} \frac{d T}{d \dot{q}}-\frac{d}{d q}(T-V)=0,
$$

is one to which we must always be alive in dealing with this subject. 


\section{CHAPTER X.}

THE CALCULATION OF THE MEAN LAGRANGIAN FUNCTION.

79. Since we can observe and regulate the forces of the types of the "controllable" coordinates we can determine how they depend upon the values of these coordinates and then by means of the expression (I55) calculate all those terms in the mean Lagrangian function which involve such coordinates. There may however be some terms in the Lagrangian function which do not involve these quantities and if we require these we must determine them by other considerations; a large number of problems can however be solved even though we do not know the values of these terms.

To get some idea of the different kinds of terms which may exist in the Lagrangian function let us consider the energy of a system consisting of a large number of molecules. In the expression for the energy we can calculate all the terms involving the coordinates which fix the electric, magnetic or elastic configuration of the system, and in the terms depending upon the strain coordinates we may include those terms which involve the average distance between the molecules. There may however be some terms left which 
each molecule contributes independently of its neighbours and which do not involve any of the controllable coordinates. The sum of these contributions will be proportional to the number of the molecules and must also be a function of the temperature, because the mean state of the system is fixed by the controllable coordinates and the temperature, and the mean kinetic energy must therefore be a function of these quantities. By hypothesis the terms we are considering do not involve the controllable coordinates, so that the only quantity they can depend upon is the temperature. The potential energy of the molecules may also contribute terms to the Lagrangian function which do not involve the controllable coordinates and which therefore we cannot calculate by equation (155). For the purposes for which we use the Lagrangian function all that we require to know about it is the change in its value when the system is changed in some definite way. Now if we measure the amount of heat absorbed or evolved when the change takes place and know the change, if any, which takes place in the kinetic energy, we can calculate the alteration in the part of the potential energy which is independent of the controllable coordinates.

The methods of calculating the mean value of the Lagrangian function will be best illustrated by working out some particular cases. Let us begin with that of a perfect gas.

Mean value of the Lagrangian function for a perfect gas.

8o. Let us suppose that unit mass of the gas is enclosed in a cylinder furnished with a piston, whose distance from the base of the cylinder is represented by the coordinate $x$, then since the pressure of the gas is a force tending to alter 
the value of $x$, the mean Lagrangian function for the system of molecules forming the gas must involve the coordinate $x$.

If $H$ denotes the mean value of the Lagrangian function of the system, the mean value of the force of type $x$ produced by the system when in a steady state is by Lagrange's equations

$$
\frac{d H}{d x} .
$$

Since there is equilibrium between the pressure due to the gas and the external pressure

$$
\frac{d H}{d x}=A p,
$$

where $p$ is the pressure of the gas and $A$ the area of the piston.

But if the gas obeys Boyle's law

$$
p=\frac{R \theta}{v},
$$

where $v$ is the volume of unit mass of the gas, $\theta$ the absolute temperature and $R$ a constant such that $R \theta$ equals the square of the velocity of sound in the gas.

Now

$$
A=\frac{d v}{d x}
$$

so that

$$
\frac{d H}{d x}=\frac{R \theta}{v} \frac{d v^{\prime}}{d x} .
$$

Integrating this equation we have in so far as $H$ depends upon $v$ and $\theta$,

$$
H=R \theta \log \frac{v}{v_{0}}+f(\theta)
$$

where $v_{0}$ is an arbitrary constant and $f(\theta)$ an arbitrary function of $\theta$, which does not involve $x$. It corresponds to the 
part of the kinetic energy which depends entirely upon unconstrainable coordinates. We shall find in the course of this work that a great many problems can be solved without a knowledge of the value of $f(\theta)$. As far as $f(\theta)$ is linear it may be included in the first term, as we may regard $v_{0}$ as quite arbitrary.

The expression ( 157 ) will give the value of the mean Lagrangian function so far as it involves $x$, it also includes that part of the kinetic energy which is expressed entirely in terms of unconstrainable coordinates, for this can be included in the term $f(\theta)$; to complete its value we nust subtract from it $u$ the potential energy of unit mass of the gas when its particles are infinitely distant from each other, as this is the part of the potential energy which depends upon uncontrollable coordinates.

Thus for unit mass of the gas

$$
H=R \theta \log \frac{v}{v_{0}}+f(\theta)-z
$$

or if $\rho$ be the density of the gas

$$
H=R \theta \log \frac{\rho_{0}}{\rho}+f(\theta)-z .
$$

We shall see later on, when we consider the phenomenon of evaporation, that $f(\theta)$ is of the form

$$
A \theta+B \theta \log \theta \ldots \ldots \ldots \ldots \ldots . . .\left(15^{8}\right) \text {. }
$$

The value of $H$ for a mass $m$ of gas whose density is $\rho$ is given by the equation

$$
H=m R \theta \log \frac{\rho_{0}}{\rho}+m f(\theta)-m w: \ldots \ldots . . .(\mathbf{I} 59) .
$$

This is the Lagrangian function for the gas itself; when an external pressure acts upon it we must add to this value the mean Lagrangian function of the system producing the pressure. We may suppose that this system is a weight 
placed upon the piston, the variable part of the potential energy of this is, if $V$ be the volume occupied by the gas

$$
p V \text {. }
$$

So that its mean Lagrangian function is

$$
-p V
$$

and the Lagrangian function of the two systems is therefore

$$
m R \theta \log \frac{\rho_{0}}{\rho}+m f(\theta)-m w-p V \ldots \ldots \ldots \text { (г60). }
$$

Mean value of the Lagrangian function for a liquid or solid.

8r. We must now proceed to find the mean value $H$ of the Lagrangian function for a liquid or solid. Let us suppose that we have a piston whose distance from a fixed plane is $x$ pressing upon a bar of the substance.

Then we have by Lagrange's equations when the motion is steady

$$
\begin{aligned}
& \frac{d H}{d x}=\text { mean force tending to increase } x \text { produced by } \\
& \text { the substance, }
\end{aligned}
$$

so that

$$
\frac{d^{2} H}{d \theta d x}=\alpha\left(\frac{d p}{d \theta}\right)_{v \text { constant }},
$$

where $p$ is the pressure required to balance this force and $a$ the area of the cross section of the bar. The differential coefficient $d p / d \theta$ is obtained on the supposition that the volume is constant.

Since

$$
\begin{gathered}
a d x=d v, \\
\frac{d^{2} H}{d \theta d v}=\left(\frac{d p}{d \theta}\right)_{v \text { constant }}
\end{gathered}
$$

we have 
Thus

$$
\begin{aligned}
\frac{d H}{d v} & =\int_{0}^{\theta}\left(\frac{d p}{d \theta}\right)_{v \text { constant }} d \theta \\
& =\theta \beta,
\end{aligned}
$$

where $\beta$ is the mean value of $(d p / d \theta)$ between zero and $\theta$.

Thus

$$
\begin{aligned}
& H=\theta \int_{v_{0}}^{v} \beta d v+f_{1}(\theta) \\
= & \theta \gamma+f_{1}(\theta) \text { say. }
\end{aligned}
$$

Where $f_{1}(\theta)$ is an arbitrary function of the temperature, it is unnecessary to add an arbitrary function of $v$ on integration as this will be included in the potential energy due to strain.

If the mass of the substance is unity

$$
v=\frac{\mathbf{I}}{\sigma},
$$

where $\sigma$ is the density, so that in the expression for $H$ for unit mass of the substance there are the terms

$$
-\theta \int_{\sigma_{0}}^{\sigma} \frac{\beta}{\sigma^{2}} d \sigma+f_{1}(\theta)
$$

From this we must subtract $w^{\prime}$ the potential energy of unit mass of the substance. Thus in the Lagrangian function for a mass $m$ of the substance there are the terms

$$
-m \theta \int_{\sigma_{0}}^{\sigma} \frac{\beta}{\sigma^{2}} d \sigma+m f_{1}(\theta)-m w^{\prime}
$$

If there is any external pressure we must add to this the expression for the mean value of the Lagrangian function of the system producing this pressure. This, as in the case of the gas, will be

$$
-p V^{\prime}
$$

where $p$ is the external pressure and $V^{\prime}$ the volume of the solid or liquid. Adding this term we get

$$
H=-m \theta \cdot \int_{\sigma_{0}}^{\sigma} \frac{\beta}{\sigma^{2}} d \sigma+m f_{\imath}(\theta)-m \boldsymbol{\prime}^{\prime}-p V^{\prime} \text { (I6I). }
$$


It must be remembered that we have only calculated the value of the Lagrangian function in the simplest case when the body is in a steady state, when it is free from all strain except that inseparable from the body at the temperature we are considering, and when it is neither electrified nor magnetized. The change in the Lagrangian function due to any additional strain or to electrification or mag. netization can be at once determined by finding the energy required to establish this particular condition. For example, the change in $H$ produced by statical electrification equals minus the potential energy of the electrical distribution, the change due to any system of electric currents flowing through solids or liquids is the kinetic energy due to this distribution of currents, and can be calculated by the ordinary formulae of electrokinetics.

82. The problems which we shall now proceed to solve, making use of the principle that the mean value of the Lagrangian function is stationary, are those which can often be solved on thermodynamical principles by using the condition that the value of the entropy of the system is stationary. The value of $H$ must therefore be closely connected with that of the entropy, and in fact we see from its value for a perfect gas in equilibrium under external pressure that, with the exception of the term $p V$, those terms in $H$ which depend upon the controllable coordinates occur also in the expression for the entropy. It seems however preferable to use the function $H$ which has a direct dynamical significance, rather than the entropy which depends upon other than purely dynamical considerations. 


\section{CHAPTER XI.}

\section{EVAPORATION.}

83. WE shall now go on to apply the principle that the value of $H$ is stationary to solve some special problems in Physics. The first problem we shall consider is that of finding the state of equilibrium when a given mass of some liquid is placed in a closed vessel from which the air has been exhausted; some of the liquid will be vaporized and we wish to find how far the vaporization will proceed before equilibrium is obtained. This of course is equivalent to finding the density of a vapour when in equilibrium in presence of the liquid.

Let $v, v^{\prime}$ be the volumes occupied by the vapour and liquid respectively, $\xi$ the mass of the vapour, $\eta$ that of the liquid, the rest of the notation being the same as that used in $\$ 80$ and $\$ 81$.

Then assuming that the vapour obeys Boyle's law we see from equation $\left(\mathrm{r}_{5}^{8}\right)$ that the vapour contributes to the expression for $H$ for the whole system the terms

$$
\xi R \theta \log \frac{v \rho_{0}}{\xi}+\xi f(\theta)-\xi z \ldots \ldots \ldots \ldots \ldots(162),
$$

since $\rho$ the density of the vapour equals $\xi / v$. 
From equation (I6I) we see that the liquid when it is free from surface tension, electrification and the like, furnishes to the same expression the terms

$$
-\eta \theta \int_{\sigma_{0}}^{\sigma} \frac{\beta}{\sigma^{2}} d \sigma+\eta f_{1}(\theta)-\eta z c^{\prime} \ldots \ldots \ldots . . .\left(\mathbf{1} 6_{3}\right) .
$$

Thus $H$ the mean value of the Lagrangian function for both the liquid and vapour is the sum of $(162)$ and $(163)$ so that we have

$$
\begin{aligned}
H=\xi R \theta & \log \frac{v \rho_{0}}{\xi}+\xi f(\theta)+\eta f_{1}(\theta) \\
& -\eta \theta \int_{\sigma_{0}}^{\sigma} \frac{\beta}{\sigma^{2}} d \sigma-\xi w-\eta w^{\prime}
\end{aligned}
$$

When there is equilibrium the value of $H$ has by the Hamiltonian principle $(\$ 75)$ a stationary value, so that in this state no small change can affect the value of the right hand side of equation (164).

The small change which we shall suppose to take place is that which occurs when the mass of the vapour is increased by a small amount $\delta \xi$ while the mass of the liquid is diminished by the same amount. The change in $H$ is

$$
{ }_{d \xi}^{d H} \delta \xi
$$

so that when there is equilibrium we have by the Hamiltonian principle

$$
\frac{d H}{d \xi}=0 .
$$

When $\delta \xi$ of the liquid is vaporized the volume of the liquid diminishes by $\delta \xi / \sigma$ so that we have

$$
\frac{d v^{\prime}}{d \xi}=-\frac{\mathbf{I}}{\sigma}
$$


and since the volume of the vapour and liquid remains constant

and therefore

$$
\frac{d}{d \xi}\left(v+v^{\prime}\right)=0,
$$

Now

$$
\frac{d v}{d \xi}=\frac{\mathbf{I}}{\sigma}
$$

$$
\begin{aligned}
\frac{d H}{d \xi}=R \theta \log \frac{v \rho_{0}}{\xi} & -R \theta+\xi R \theta \frac{\mathrm{I}}{v} \frac{d v}{d \xi} \\
& +f(\theta)-f_{1}(\theta)+\gamma \theta-w+w^{\prime}
\end{aligned}
$$

where for brevity $\gamma$ is written instead of

$$
-\int_{\sigma_{0}}^{\sigma} \frac{\beta}{\sigma^{2}} d \sigma
$$

Substituting for $d v / d \xi$, its value we have

$$
\frac{d H}{d \xi}=R \theta \log \frac{v \rho_{0}}{\xi}+\xi \frac{R \theta}{v \sigma}-\left(w-w^{\prime}\right)+\psi(\theta) \ldots\left(\text { I }_{5}\right),
$$

where $\psi(\theta)$ is written for

$$
(\gamma-R) \theta+f(\theta)-f_{1}(\theta)
$$

a quantity which does not involve $\xi$. Since $\xi / \nu=\rho$ we may write $(165)$ as

$$
\frac{d H}{d \xi}=R \theta \log \frac{\rho_{0}}{\rho}+R \theta \frac{\rho}{\sigma}-\left(w-w^{\prime}\right)+\psi(\theta) .
$$

Since $d H / d \xi$ vanishes in the state of equilibrium we have then

$$
\begin{gathered}
R \theta \log \frac{\rho_{0}}{\rho}=-R \theta \frac{\rho}{\sigma}+\left(w-w^{\prime}\right)-\psi(\theta) \ldots(\mathrm{I} 66), \\
\rho=\rho_{0} \epsilon^{\frac{\rho}{\sigma}+\frac{\psi(\theta)}{R \theta}} \epsilon-\frac{\left(w-w^{\prime}\right)}{R \theta},
\end{gathered}
$$

or

since $\rho / \sigma$ is very small we may write this as

$$
\rho=\phi(\theta) \epsilon^{-\frac{(w-w)}{K \theta}}
$$

where $\phi(\theta)$ is some function of $\theta$. 
Bertrand (Thermodynamique, p. 93) has shown that the results of Regnault's experiments on the vapour pressures of different liquids can be represented by the following expressions, $p$ being the pressure in millimetres of mercury:

water; $\quad \log p=17.44324-2795 / \theta-3.8682 \log \theta$, ether; $\quad \log p=\mathrm{I} 3.423 \mathrm{II}-\mathrm{I} 729 / \theta-\mathrm{I} \cdot 9787 \log \theta$, alcohol; $\quad \log p=2$ I. $^{*} 44^{686}-2743 / \theta-4^{22248} \log \theta$, chloroform; $\log p=19.29792-2179 / \theta-3.91583 \log \theta$, bisulphide of carbon $\log p=\mathrm{r} 2 \cdot 58852-\mathrm{r} 684 / \theta-\mathrm{r} \cdot 7689 \log \theta$.

This form of expression was originally used by Dupré: (Théorie Mécanique de la Chaleur, p. 97.)

The coefficient of $r / \theta$ in each of these expressions is nearly $\lambda_{0} / R$, where $\lambda_{0}$ is the latent heat of the substance at the absolute zero of temperature. This is the term $(w-w) / \theta$ in our expression (r66) and $w-w^{\prime}$ is the latent heat at absolute zero, hence by comparing the other terms in these expressions we see that $f(\theta)$ must be of the form

$$
A \theta+B \theta \log \theta .
$$

84. We can by the aid of the preceding formulae very easily determine the effect upon the vapour pressure of any slight change in the physical condition of the liquid or vapour.

Let us suppose that the physical conditions are so changed that the mean Lagrangian function exceeds the value we have hitherto assumed for it by $\chi$. Then instead of equation (167) we have evidently

$$
R \theta \log \frac{\rho_{0}}{\rho}+R \theta \frac{\rho}{\sigma}+\psi(\theta)-\left(w-w^{\prime}\right)+\frac{d \chi}{d \xi}=0,
$$

so that $\delta \rho$ the change in the vapour density due to the cause

T. D. 
which produced the change $\chi$ in the mean Lagrangian function is given by the equation

$$
\begin{aligned}
-R \theta \frac{\delta \rho}{\rho}+R \theta \frac{\delta \rho}{\sigma}+\frac{d \chi}{d \xi}=0, \\
\delta \rho=\frac{\mathrm{I}}{R \theta} \frac{\rho \sigma}{\sigma-\rho} \frac{d \chi}{d \xi} \ldots \ldots \ldots \ldots(\mathrm{I} 68),
\end{aligned}
$$

or

so that if $\chi$ increases with $\xi$ the vapour pressure in the state of equilibrium is increased, while if $\chi$ diminishes as $\xi$ increases the equilibrium vapour pressure is diminished. This very important principle is a particular case of the more general one that; when the physical environment of a system is slightly changed and the consequent change in the mean Lagrangian function increases as any physical process goes on, then this process will have to go on further in the changed system before equilibrium is reached than in the unchanged one, while if the change in the mean Lagrangian function diminishes as the process goes on it will not have to proceed so far. We shall have numerous examples of this principle in the course of the following pages.

85. Let us now consider the effect of surface tension upon the vapour pressure. In order to take a definite case let us suppose that the liquid is a spherical drop. It will possess in consequence of surface tension potential energy proportional to its area, and as the area of the drop diminishes as the water evaporates the energy due to the surface tension changes, and since anything which causes the energy to change as evaporation goes on alters the state of equilibrium, the vapour pressures when there is equilibrium in this case cannot be the same as when evaporation produces no change in the area and therefore no change in the energy due to surface tension. 
If $a$ be the radius of the drop and $T$ the energy per unit area due to surface tension then in the expression for the potential energy of the liquid there will be in addition to the terms we have already considered the term

$$
4 \pi a^{2} T \text {, }
$$

and therefore in the mean Lagrangian function for the liquid and vapour the additional term

$$
-4 \pi a^{2} T \text {. }
$$

So that with our previous notation

Now

$$
\begin{gathered}
\chi=-4 \pi a^{2} T . \\
\frac{\mathrm{r}}{a} \frac{d a}{d \xi}=\frac{\mathrm{r}}{3} \frac{\mathrm{I}}{v^{\prime}} \frac{d v^{\prime}}{d \xi},
\end{gathered}
$$

and therefore

$$
=-\frac{\mathbf{r}}{3 z^{\prime} \boldsymbol{\sigma}},
$$

hence

$$
\frac{d a}{d \xi}=-\frac{\mathrm{x}}{4 \pi a^{2} \boldsymbol{\sigma}}
$$

and therefore

$$
\frac{d \chi}{d \xi}=\frac{2 T}{a \sigma} .
$$

So that if $\delta \rho$ be the change in the vapour density produced by the surface tension we have by equation (I68)

$$
\delta \rho=\frac{2 \rho}{\sigma-\rho} \frac{T}{a} \frac{\mathrm{r}}{R \theta} \ldots \ldots \ldots \ldots \ldots(\mathrm{r} 7 \mathrm{0}),
$$

and if $\delta p$ be the change in the vapour pressure, since

we have by $(\mathrm{r} 70)$

$$
\delta p=R \theta \delta \rho,
$$

$$
\delta p=\frac{2 \rho}{\sigma-\rho} \frac{T}{a} \ldots \ldots \ldots \ldots \ldots \ldots(\mathrm{r} 7 \mathrm{x}) .
$$

This agrees with the formula given by Sir William Thomson in the Proceedings of the Royal Society of

$$
\text { I I }-2
$$


Edinburgh, Feb. 7, 1870, and quoted in Maxwell's Theory of Heat, $5^{\text {th }}$ edit. p. 290.

If we take the case of a drop of water $\frac{1}{10}$ of a millimetre in radius, then if the temperature is about $10^{\circ} \mathrm{C}$. we have by ( 170 ), since $R \theta$ for water vapour is about $1{ }^{1} 3 \times 10^{9}$

$$
\frac{\delta \rho}{\rho}=\frac{200}{\mathrm{I} \cdot 3 \times 10^{9}} \times T,
$$

since $T=8 \mathrm{I}$ we have

$$
\frac{\delta \rho}{\rho}=\mathrm{I} \cdot 2 \times 10^{-5} .
$$

We see that the energy due to surface tension makes the Lagrangian function increase as evaporation goes on, so that by the principle given at the end of $\S 84$, the effect of it will be to make evaporation go on further than it otherwise would.

If we have the water in narrow capillary tubes then when it evaporates the area of the surface of contact of the tube with water is diminished but that of the surface of contact of the tube with air is increased. Since the surface tension of the surface of separation of the tube and air is greater than that of the tube and water, the potential energy due to surface tension increases as evaporation goes on, thus the mean Lagrangian function diminishes as the liquid evaporates, so that by the principle of $\S 84$ the effect of surface tension in this case will be to stop evaporation and promote condensation. We can easily shew that if $a$ is the radius of the tube then in this case

$$
\delta p=-\frac{2 \rho}{\sigma-\rho} \cdot \frac{T}{a} .
$$

86. Effect of a charge of electricity on the vapour pressure. We can by the use of formula (168) 
find the effect on the vapour density of electrifying the liquid. If the charge $e$ is given to the liquid which we shall suppose spherical and of radius $a$, the potential energy is increased by

$$
{ }_{2} \mathrm{I} \frac{e^{2}}{a},
$$

where $K$ is the specific inductive capacity of the surrounding medium.

The mean Lagrangian function of the liquid and its vapour is diminished by this amount. Blake's experiments on the evaporation of electrified liquids (Wiedemann's Elektricitit, IV. p. I 2 I 2) show that $e$ remains constant as the liquid evaporates, in other words that the vapour proceeding from the electrified liquid is not electrified. Thus the new term $-e^{2} / 2 K a$ in the mean Lagrangian function diminishes as the liquid evaporates and therefore by the principle of $\$ 84$ evaporation will not go on so far as before, that is the vapour density when there is equilibrium will be diminished by electrifying the liquid.

We can easily calculate by equation (I68) the amount of this diminution.

In this case

$$
X=-{ }_{2} \mathrm{I} \frac{e^{2}}{a},
$$

and therefore

$$
\frac{d \chi}{d \xi}=\frac{1}{2} \frac{e^{2}}{K a^{2}} \frac{d a}{d \xi}
$$

substituting the value of $d a / d \xi$ given by (I69) we have

$$
\frac{d \chi}{d \xi}=-\frac{\mathbf{I}}{8 \pi K} \frac{e^{2}}{a^{4} \sigma},
$$

so that if $\delta \rho$ be the change in the vapour density produced by the electrification we have by (I68)

$$
\delta \rho=-\frac{\mathrm{I}}{R \theta} \frac{\rho}{\sigma-\rho} \stackrel{e^{2}}{8 \pi K a^{4}}
$$


To calculate the magnitude of this effect let us suppose $K=\mathrm{I}$; then $e / a^{2}$ is the electric force just outside the sphere, and this cannot exceed a certain value, otherwise the insulating power of the air would break down and the electricity escape. The maximum value of $e / a^{2}$ when the sphere is surrounded by air at the atmospheric pressure is about 120 in electrostatic measure : and as $\sigma$ is unity, $\rho$ a small fraction, the maximum alteration in the vapour density will be given by the equation

$$
\frac{\delta \rho}{\rho}=-\mathrm{I} \cdot 44 \times 10^{4} \times \frac{\mathrm{I}}{8 \pi R \theta},
$$

now $R \theta$ for water vapour at $10^{\circ} \mathrm{C}$. is about $\mathrm{I} 3 \times 10^{\circ}$, so. that

$$
\frac{\delta \rho}{\rho}=-4 \times 10^{-7},
$$

this value will be independent of the size of the drop. Comparing equations (I7O) and (I72) we see that the maximum effect due to electrification is about equal in magnitude though opposite in sign to that due to a curvature of $I / 4$ of a centimetre.

The effect of electrification is to diminish the vapour density when there is equilibrium between the liquid and the vapour, it therefore increases the tendency of the vapour to deposit on the liquid. We should therefore expect an electrified drop of rain to be larger than an unelectrified one, so that this effect may help to produce the large drops of rain which fall in thunderstorms.

87. Effect of an electric field upon the vapour pressure. Electricity also produces an effect upon the vapour pressure when the drop is not charged but merely placed in an electric field. Let us suppose that the field is due to a charge of electricity $e$ collected at a point 
$P$, let the radius of the drop of water which we shall suppose spherical be $a$ and let $f$ be the distance of the centre from $P$. Then (Maxwell's Electricity and Magnetism, Vol. I. p. 232), the potential energy due to the mutual action of the electrified point and the drop of water is

$$
-\frac{\mathrm{I}}{2 K} \frac{e^{2} a^{3}}{f^{2}\left(f^{2}-a^{2}\right)}
$$

so that the increase in the mean Lagrangian function is

$$
\frac{\mathrm{I}}{2 K} \frac{e^{2} a^{3}}{f^{2}\left(f^{2}-a^{2}\right)},
$$

and therefore by (168) the cliange $\delta \rho$ in the density of the vapour when in a state of equilibrium is given by the equation

$$
\frac{\delta \rho}{\rho}=\frac{\mathrm{I}}{R \theta} \frac{\boldsymbol{\sigma}}{\sigma-\rho} \frac{\mathrm{I}}{K}\left\{\frac{3}{2} \frac{e^{2} a^{2}}{f^{2}\left(f^{2}-a^{2}\right)}+\frac{e^{2} a^{4}}{f^{2}\left(f^{2}-a^{2}\right)^{2}}\right\} \frac{d a}{d \xi} .
$$

Substituting for $d a / d \xi$ from (I69) we have

$$
\frac{\delta \rho}{\rho}=-\frac{\mathbf{I}}{R \theta K} \frac{e^{2}}{\boldsymbol{\sigma}-\rho}\left\{\frac{3}{2} f^{2} \frac{\mathbf{I}}{\left(f^{2}-a^{2}\right)}+\frac{a^{2}}{f^{2}\left(f^{2}-a^{2}\right)^{2}}\right\} \frac{\mathbf{I}}{4^{\pi}},
$$

if $a$ be small compared with $f$ then approximately

$$
\frac{\delta \rho}{\rho}=-\frac{3}{8 \pi} \frac{\mathrm{I}}{R K \theta} \frac{\mathrm{I}}{\boldsymbol{\sigma}-\rho} \frac{e^{2}}{f^{4}} .
$$

Now $e / K f^{2}$ is the force at the centre of the drop due to the electrified point, calling this $F$ and remembering that $\sigma$ is large compared with $\rho$ we have

$$
\frac{\delta \rho}{\rho}=-\frac{3 K F^{2}}{8 \pi \sigma R \theta}
$$

so that the effect of electrification on a neighbouring body is again to diminish the vapour density in the state of equilibrium. The formula (173) will evidently hold even though the field is not due to an electrified point provided 
$F$ the force at the drop does not vary much in a distance comparable with the radius of the drop.

88. Effect of strain upon vapour pressure. We shall now investigate how the vapour pressure depends upon the state of compression of the liquid. Let us suppose that the pressure $p$ acts upon the liquid, then if $k$ be the modulus of resistance to compression, the potential energy possessed by the liquid in virtue of this strain is

$$
\frac{\mathrm{I}}{2} \frac{p^{2}}{k} \frac{\eta}{\sigma}
$$

so that

$$
\frac{d \chi}{d \xi}=\frac{\mathbf{I}}{2} \frac{p^{2}}{\sigma k},
$$

and therefore by equation ( 168 )

$$
\frac{\delta \rho}{\rho}=\frac{\mathbf{I}}{2} \frac{\mathbf{I}}{R \theta} \frac{\mathbf{I}}{\sigma-\rho} p^{2} \ldots \ldots \ldots \ldots(\mathbf{I} 74),
$$

or approximately

$$
\frac{\delta \rho}{\rho}=\frac{\mathrm{I}}{2 R \theta} \frac{p^{2}}{\sigma k}
$$

for water $k=2.2 \times 10^{11 "}$, so that the effect on the vapour pressure of the 'compression due to the pressure of rcoo atmospheres is at the temperature of $15^{\circ} \mathrm{C}$. given by

$$
\frac{\delta \rho}{\rho}=\frac{\mathrm{I}}{5^{6}} ;
$$

for ether the effect would be given by

$$
\frac{\delta \rho}{\rho}=\frac{I}{5} \text { approximately. }
$$

In the next paragraph we shall consider another effect due to pressure which except for exceedingly large pressures is larger than the one we have just been considering. 
89. Effect of the presence of a gas having no chemical action on the water vapour on the equilibrium vapour pressure. It is generally believed that the equilibrium vapour pressure of water depends only upon the temperature of the water and not upon the pressure produced by an indifferent gas, that it is for example the same in a vacuum as under atmospheric pressure. If however we remember that when a portion of the liquid evaporates the air above it must expand and do work we shall see that this cannot be the case, but that since evaporation is accompanied by a diminution in the density of the air, and therefore by an increase in its mean Lagrangian function, it must by the principle of $\S 84$ go on further when air is present than in a vacuum, so that the vapour density will be increased by the presence of the air. We shall now go on to investigate the magnitude of this increase and shall consider two cases. In the first case we shall suppose that the air and liquid are placed in a closed vessel whose volume remains constant.

Let $\xi, \eta, \zeta$ be the masses of the vapour, water, and air respectively; $w, w_{1}, w_{2}$, the mean potential energy of unit mass of each of these substances respectively.

Then the mean Lagrangian function for the water vapour is

$$
\xi R \theta \log \frac{{ }^{\prime} \rho_{0}}{\xi}+\xi f(\theta)-\xi w,
$$

where $v$ is the volume of the vessel above the liquid.

The mean Lagrangian function for the water is by equation $(\mathbf{r} 6 \mathbf{I})$

$$
\eta \gamma \theta+\eta f_{1}(\theta)-\eta w_{1}
$$

and the mean Lagrangian function for the air is

$$
\zeta R_{2} \theta \log \frac{v \rho_{0}}{\zeta}+\zeta f_{2}(\theta)-\zeta w_{2} .
$$


Thus when a quantity $d \xi$ of the liquid evaporates, since $d v / d \xi=\mathrm{I} / \sigma$, the condition that $a H / d \xi=0$ gives $R \theta \log \frac{\eta \rho_{0}}{\xi}+\frac{\xi R \theta}{\eta \sigma}-R \theta+f(\theta)-f_{1}(\theta)-\gamma \theta-\left(w-w w_{1}\right)+\frac{\zeta R_{2} \theta}{\sigma v}=0$.

Now if $\delta \rho$ be the change in $\rho$ due to the presence of the air, $\delta w_{1}$ the corresponding change in $z_{1}$, we have by this equation

$$
-\frac{R \theta \delta \rho}{\rho}+\frac{R \theta \delta \rho}{\sigma}+\delta w_{1}+\frac{\zeta R_{2} \theta}{\sigma v}=0 \ldots \ldots(\mathrm{I} 76) .
$$

The presence of the air will increase the pressure and so cause the liquid to be more compressed than it would be if the air were away, so that $w_{1}$ will be increased by the presence of the air. If $\delta e$ be the compression due to the pressure $p^{\prime}$ of the air, and $p$ the pressure due to the water vapour, $\delta z \psi_{1}$ will be proportional to $\left(\not p+p^{\prime}\right) \delta e$ and unless the pressure due to the air amounts to many thousands of atmospheres this term will be very small compared with

which is equal to $p^{\prime} / \sigma$.

$$
\frac{\zeta R_{2} \theta}{\sigma v}
$$

Hence we may write equation (I 76$)$ as

$$
-\frac{R \theta \delta \rho}{\rho}+\frac{R \theta \delta \rho}{\sigma}+\frac{p^{\prime}}{\sigma}=0,
$$

or, since $\sigma$ is very large compared with $\rho$,

$$
R \theta \frac{\delta \rho}{\rho}=\frac{p^{\prime}}{\sigma} \ldots \ldots \ldots \ldots \ldots \ldots . . . . .(\mathrm{I} 77) .
$$

Now if $\delta p$ be the change in the pressure of the water vapour due to the presence of the air

$$
R \theta \delta \rho=\delta p,
$$

so that equation (r77) becomes

$$
\frac{\delta p}{\rho}=\frac{p^{\prime}}{\sigma} .
$$


But if $\rho^{\prime}$ is the density of steam when the pressure is $p^{\prime}$

$$
\frac{p}{\rho}=\frac{p^{\prime}}{\rho^{\prime}},
$$

so that equation (177) becomes

$$
\frac{\delta p}{p}=\frac{\rho^{\prime}}{\sigma}
$$

and thus the alteration in the vapour pressure produced by an external pressure of an atmosphere is given by the equation

$$
\begin{aligned}
\frac{\delta p}{p} & =\frac{\text { density of steam at atmospheric pressure }}{\text { density of water }} \\
& =\frac{\mathrm{I}}{\mathrm{I} 200} \text { at } 0^{\circ} \mathrm{C} .
\end{aligned}
$$

So that for each atmosphere of pressure the vapour pressure of water is increased by about one part in twelve hundred. For ether the increase would be about one part in 220.

90. 'The other case we shall consider is when the pressure acting on the system remains constant. We shall use the same notation as before. The only change we shall have to make in the mean Lagrangian function is to add to it that of the system producing the steady external pressure. We may suppose in order to fix our ideas that this system is a quantity of mercury placed on the piston, which may be supposed to move vertically up and down, then if $P$ be the steady pressure per unit area the potential energy is equal to

$$
P\left(v+v^{\prime}\right)
$$

so that the mean Lagrangian function of this system is

$$
-P\left(v+v^{\prime}\right)
$$

where $v^{\prime}$ is the volume of the liquid. 
gives

The condition $\quad \frac{d H}{d \xi}=0$

$$
\begin{aligned}
& R \theta \log \frac{\rho_{0}}{\rho}-R \theta+\frac{\xi R \theta}{v} \frac{d v^{\prime}}{d \xi}+f(\theta)-f_{1}(\theta)-\gamma \theta-\left(u-w_{1}\right) \\
& +\frac{\zeta R_{2} \theta d v}{v} \frac{d v}{d \xi}-P\left(\frac{d v}{d \xi}+\frac{d v^{\prime}}{d \xi}\right)=0 \text {. } \\
& \frac{\xi R \theta}{v}=p \\
& \frac{\zeta R_{\mathrm{e}} \theta}{v}=p^{\prime}
\end{aligned}
$$

where $p$ and $\not p^{\prime}$ are the pressures due to the water vapour and air respectively.

Since

and

$$
\begin{aligned}
P & =p+p^{\prime} \\
\frac{d v^{\prime}}{d \xi} & =-\frac{\mathrm{r}}{\sigma}
\end{aligned}
$$

the above equation reduces to

$$
R \theta \log \frac{\rho_{0}}{\rho}-R \theta+f(\theta)-f_{1}(\theta)-\gamma \theta-\left(w-w_{1}\right)+\frac{P}{\sigma}=0,
$$

or if $\delta \rho$ be the change produced by the external pressure,

or

$$
\begin{aligned}
& R \theta \frac{\delta \rho}{\rho}=\frac{P}{\sigma} \\
& \frac{\delta p}{\rho}=\frac{P}{\sigma}
\end{aligned}
$$

a similar result to the one we obtained before.

We see from this result that (apart from any other cause) rain drops will form more easily when the barometer is low than when it is high.

Regnault's experiments seem to show that the vapour pressure in a vacuum is greater by nearly 5 per cent. than when there is air at atmospheric pressure above the liquid (Wullner's Lehrbuch der Physik, III. p. 703), but he attributed this difference to the condensation of the liquid on the sides 
of the vessel; the absorption of the air by the liquid might also tend to produce an effect in this direction, though, as the following investigation will show, to nothing like the extent of 5 per cent.

91. Effect of absorbed air on the vapour pressure. When the liquid contains some gas diffused through its volume which remains behind when it evaporates, the evaporation of the liquid will cause the volume occupied by the gas to diminish and its density to increase. Thus by (157) the mean value of its Lagrangian function will diminish as evaporation goes on, so that by $\$ 84$ the presence of the gas dissolved throughout the volume will diminish the equilibrium vapour pressure.

Let $\epsilon$ be the mass of the dissolved gas, $v^{\prime}$ the volume of the liquid in which it is dissolved, then the Lagrangian function of the gas is

$$
\epsilon R^{\prime} \theta \log \frac{v^{\prime} \rho_{0}}{\epsilon}-\epsilon z \ell^{\prime}+f^{\prime}(\theta) \ldots \ldots \ldots(\mathrm{x} 78),
$$

where $w^{\prime}$ is the intrinsic potential energy of unit mass of the dissolved gas.

The expression ( 178 ) is the quantity we denoted by $\chi$ in $\S 84$. 'The only variable in $\chi$ which involves $\xi$ is $v^{\prime}$ and $d \tau^{\prime} / d \xi=-I / \sigma$, so that we have

and therefore by (168)

$$
\frac{d \chi}{d \xi}=-\frac{R^{\prime} \theta \epsilon}{v^{\prime} \sigma}
$$

$$
R \theta \frac{\delta \rho}{\rho}=-\frac{\mathbf{I}}{\sigma-\rho} \frac{R^{\prime} \theta \epsilon}{v^{\prime}} \ldots \ldots \ldots \ldots . . . .(\mathrm{I} 79) .
$$

If $\delta p$ be the increase in the vapour pressure caused by the dissolved gas, $P$ the pressure this gas would produce if it were free from the liquid and filled the volume $v^{\prime}$, then since

$$
R \theta \delta \rho=\delta p
$$


and

$$
\frac{R^{\prime} \theta \epsilon}{v^{\prime}}=P,
$$

equation (I79) may be written

$$
\delta p=-\frac{\rho}{\sigma-\rho} P .
$$

So that since $\rho$ is very small compared with $\sigma$ we have approximately

$$
\delta p=-\frac{\rho}{\sigma} P \ldots \ldots \ldots \ldots \ldots \ldots(\text { I } 80),
$$

or if $p$ be the vapour pressure, and $\rho$ ' the density of steam at the atmospheric pressure $\pi$, equation (180) may be written

$$
\frac{\delta p}{p}=-\frac{\rho^{\prime}}{\sigma} \frac{P}{\pi} \text {. }
$$

And since $\rho^{\prime} / \sigma$ is about I / I 200 we see that

$$
\frac{\delta p}{p}=-\frac{\mathrm{I}}{\mathrm{I} 200}(P)
$$

where $(P)$ is the pressure $P$ expressed in atmospheres.

The volumes of the various gases which one volume of water will absorb at $0^{\circ} \mathrm{C}$. under the pressure of 760 millimetres of mercury were determined by Bunsen and are given in the following table :

$$
\begin{array}{ll}
\text { Hydrogen } & .019 \\
\text { Nitrogen } & .0203 \\
\text { Air } & .0247
\end{array}
$$

Carbonic Acid $1 \cdot 79$

Chlorine

so that according to equation (180) the vapour pressure of water saturated with air will be lowered by about one part in 50,000, when saturated with carbonic acid by about I part in 660 and when with chlorine by about I part in 400 .

In this investigation we have assumed that the properties of the liquid are not altered by the presence of the gas; if they are, then we must regard $\gamma$ and $w^{\prime}$ as functions of $\epsilon$, and this would lead to the introduction of several additional 
terms into the equation for $\delta p$. We have assumed too that the gas remains behind as the liquid evaporates, as in this case the diminution of the vapour pressure is greater than if some of the gas were to be set free when the liquid evaporates.

92. The effect of dissolved salt on the vapour pressure. Van 't Hoff ("L'équilibre chimique dans les systèmes gazeux ou dissous à l'état dilué," Archives Neerlandais, xx. p. 239, I886) has pointed out that Pfeffer's experiments on the osmotic pressure produced by salts dissolved in water (Pfeffer, Osmotische Untersuchungen, Leip/ig, I877) and Raoult's experiments on the effect of. dissolved salts on the freezing point of solutions (Annales de Chimie, $6^{\text {me }}$ serie, 1v. p. 40 r), show that the molecules of a salt in a dilute solution exert the same pressure as they would exert if they were in the gaseous state at the same temperature and occupying a volume equal to that of the liquid in which the salt is dissolved, and that the pressure exerted by these molecules obeys Boyle's and Gay Lussac's law. This being so, the mean Lagrangian function for the salt dissolved in the liquid is the same as that of an equal mass of the salt in the gaseous state filling the volume occupied by the liquid. Thus if the properties of the liquid are not altered by the presence of the salt the results of the preceding section will apply, and we shall have, supposing that the salt remains behind when the liquid evaporates,

$$
\frac{\delta p}{p}=-\frac{\rho}{\sigma}(P) \ldots \ldots \ldots \ldots \ldots(\mathrm{r} 8 \mathrm{r})
$$

where $\sigma$ is the density of the liquid, $\rho$ the density of its vapour at the atmospheric pressure, $(P)$ the pressure in atmospheres which would be exerted by the dissolved salt if it were in the gaseous state.

Thus, for example, suppose that we have $n$ grammes of 
salt in a litre of the solvent, where $n$ is the molecular weight of the salt. This strength of solution is often called for brevity a strength of one equivalent per litre. This quantity of salt will by Avogadro's law produce the same pressure as 2 grammes of hydrogen per litre, that is about $\mathbf{2 2}$ atmospheres; if this quantity of salt were dissolved in water it would by equation (I8I) since $\rho / \sigma$ is about $r / 1200$ diminish the vapour pressure by about $\mathrm{I}$ part in 55 , if it were dissolved in ether, $\mathrm{C}_{4} \mathrm{H}_{10} \mathrm{O}$, where $\rho / \sigma$ is about $\mathrm{I} / 220$, then the vapour pressure would be diminished by about I part in Io, if it were dissolved in alcohol, $\mathrm{C}_{2} \mathrm{H}_{6} \mathrm{O}$, where $\rho / \sigma$ is about $\mathbf{I} / 380$, the vapour pressure would be reduced by about one part in $\mathbf{I} 7$. We see from equation $\left(18_{1}\right)$ that the diminution in the vapour pressure is proportional to the quantity of salt dissolved. We can also express the result of this equation as follows. If $P$ is the pressure due to one equivalent in grammes of the salt dissolved in a kilogramme of the solvent, then $P / \sigma$, where $\sigma$ is the density of the solvent, is the pressure in atmospheres due to one equivalent of the salt dissolved in a litre of the solvent. Hence $\frac{P}{\sigma}=\frac{2}{10^{3}} /$ (density of hydrogen at atmospheric pressure.) So that we may write equation ( $\mathrm{I} 8 \mathrm{I}$ ) as

$$
\frac{\delta p}{p}=(\text { molecular weight of solvent }) \times \mathbf{I} \times 10^{-3},
$$

where $\delta p$ is the diminution in vapour pressure when one equivalent of the salt is dissolved in a kilogramme of the solvent. Another way of expressing the same thing is that when one equivalent of the salt is dissolved in 1000 equivalents of the solvent, i.e. in I000 $\mathrm{m}$ grammes where $m$ is the molecular weight of the solvent, the diminution in the vapour pressure amounts to I part in I000, whatever be the nature of the salt or solvent. 
Since

$$
\frac{\delta p}{p}=\frac{\rho^{\prime}}{\sigma} \frac{P}{\pi},
$$

and since $P$ is directly, while $\rho^{\prime}$ is inversely, proportional to the absolute temperature, we see that the ratio $\delta p \mid p$ ought to be nearly independent of the temperature since $\sigma$ only varies very slowly with it.

93. In the preceding investigation we have assumed that the properties of the solvent were unaltered by the presence of the salt, and that all the solvent did was to enable the salt to exist in a condition in which the molecules were very far apart.

If however the properties of the solvent are altered by the presence of the salt, then we must regard $w^{\prime}$ as a function of the quantity of salt dissolved.

In this case instead of equation (179) we shall have

$$
R \theta \delta \rho\left\{\frac{\mathbf{I}}{\rho}-\frac{\mathbf{I}}{\sigma}\right\}+\delta w^{\prime}+\eta \frac{d w^{\prime}}{d \eta}+\frac{P}{\sigma}=0 \ldots . .(182),
$$

where $\delta w^{\prime}$ is the change in $w^{\prime}$ produced by the presence of the salt.

Now if $s$ be the strength of the solution, i.e. the quantity of salt in unit volume of the solvent,

$$
\eta \frac{d w^{\prime}}{d \eta}=-s \frac{d w^{\prime}}{d s}
$$

so that equation (182) becomes

$$
R \theta \delta \rho\left(\frac{\mathrm{I}}{\rho}-\frac{\mathrm{I}}{\sigma}\right)+\delta w^{\prime}-s \frac{d w^{\prime}}{d s}+\frac{P}{\sigma}=0 .
$$

If the change in $w^{\prime}$ is proportional to the strength of the solution then

$$
\delta w^{\prime}-s \frac{d w^{\prime}}{d s}=0
$$

T. D. 
In the general case the lowest power of $s$ which occurs in the expression

$$
\delta w e^{\prime}-s \frac{d w^{\prime}}{d s}
$$

is the second, so that the effect produced by the alteration of the properties of the solvent depends upon the squares and higher powers of the concentration, while the effect we investigated in the preceding section was proportional to the first power, and therefore when the solution is dilute is relatively the more important.

Raoult, Comptes Rendus 104, p. I433, has recently found that when one equivalent of a substance is dissolved in 100 equivalents of a solvent the vapour pressure is reduced by $\mathrm{r} \cdot 05$ parts in I00, which agrees very well with the results we have just obtained. 


\section{CHAPTER XII.}

\section{PROPERTIES OF DILUTE SOLUTIONS.}

94. THE effect produced on the vapour pressure of any solvent by dissolving other substances in it has been discussed in the last chapter; in this chapter we shall consider some other properties possessed by dilute solutions.

Absorption of gases by liquids. Let us suppose that we have a closed cylinder containing a gas and a liquid and that we wish to find how much of the gas will be absorbed by the liquid. In this case we have four substances to consider,

I. The liquid.

2. The vapour of the liquid.

3. The free gas.

4. The gas dissolved in the liquid.

The variation which we shall suppose to take place, and which will not by the Hamiltonian principle alter the value of $H$ when the system is in equilibrium, is that corresponding to the escape of a small quantity of gas from the liquid. This will not affect the value of the mean Lagrangian function of the vapour of the liquid, so that we may leave this 
out of account in solving this problem. Let the mass of the liquid be $\eta$, that of the free gas $\xi$, and that of the absorbed gas $\zeta$. Then using the same notation as we have hitherto employed, the mean Lagrangian function for the liquid is

$$
\eta \gamma \theta+\eta f_{1}(\theta)-\eta w_{1} \ldots \ldots \ldots \ldots . . .\left(188_{3}\right),
$$

for the free gas

$$
\xi R \theta \log \frac{v^{\prime} \rho_{0}}{\xi}+\xi f(\theta)-\xi w \ldots \ldots \ldots . .\left(\mathrm{I} 8_{4}\right),
$$

for the dissolved gas

$$
\zeta R \theta \log \frac{v^{\prime} \rho_{0}^{\prime}}{\zeta}+\zeta f^{\prime}(\theta)-\zeta w^{\prime} \ldots \ldots \ldots \ldots\left(18_{5}\right),
$$

where $v$ is the volume occupied by the free gas, and $v^{\prime}$ the volume of the liquid or that occupied by the dissolved gas, and where $z \ell^{\prime}$ and $f^{\prime}(\theta)$ are the quantities for the dissolved gas which correspond to $w$ and $f(\theta)$ for the free gas. If we denote the sum of the expressions $(183),(184)$ and $(185)$ by $H$, then by the Hamiltonian principle $H$ is stationary when the system is in equilibrium, so that if we suppose a small variation to be caused by a quantity of gas $\delta \xi$ escaping from the liquid, then we must have for equilibrium

$$
\frac{d H}{d \xi} \delta \xi=0,
$$

this is equivalent to

$$
\begin{aligned}
R \theta \log \frac{v \rho_{0}}{\xi}-R \theta & +f(\theta)-w-R \theta \log \frac{v^{\prime} \rho_{0}^{\prime}}{\zeta}+R \theta-f^{\prime}(\theta)+w^{\prime} \\
& +\frac{d}{d \xi}\left\{\eta \theta+\eta f_{1}(\theta)-\eta w_{1}\right\}=0 \ldots \ldots . .(\mathbf{r} 86) .
\end{aligned}
$$

The last term when the amount of gas absorbed is not large will be very nearly independent of the quantity of gas dissolved. Equation (186) may be written 


$$
\begin{aligned}
R \theta \log c \frac{\rho^{\prime}}{\rho}=w-w^{\prime}+ & f^{\prime}(\theta)-f(\theta) \\
& +\frac{d}{d \zeta}\left\{\eta \gamma \theta+n f_{1}(\theta)-\eta w_{1}\right\} \ldots . .(\text { I } 87),
\end{aligned}
$$

where $c$ is a constant and $\rho$ and $\rho^{\prime}$ are the densities of the free anci dissolved gas respectively. Since the temperature is constant, we see from this equation that $\rho^{\prime} / \rho$ is constant, that is, the quantity of gas in unit volume of the liquid is proportional to the density of the free gas. This is Henry's law of the absorption of gases by liquids and it has been verified by the researches of Bunsen and others. Bunsen's experiments showed that the value of the ratio $\rho^{\prime} / \rho$ depends upon the temperature, hence we see from equation ( 187 ) that $w-f(\theta)-\left\{w^{\prime}-f^{\prime}(\theta)\right\}+\frac{d}{d \zeta}\left\{\eta \gamma \theta+n f_{1}(\theta)-\eta w_{1}\right\}$ cannot be zero, otherwise $\rho^{\prime} / \rho$ would be the same at all temperatures. Thus either the properties of the free gas can not be quite the same as those of the dissolved gas, or else the properties of the water are altered by the gas dissolved in it.

95. A similar investigation will apply to the case of a solid or gas which can dissolve in two fluids which do not mix. We can prove in this way that when there is equilibrium when the fluids are shaken up together then, provided the solutions are dilute, the amount dissolved in unit volume of one fluid will bear a constant ratio to that dissolved in the same volume of the other (see Ostwald's Lehrbuch der allgemeinen Chemie, I. p. 40I).

96. The diffusion of salts through the solvent, a process which goes on until the solution acquires a definite state, can be explained by the same principles. In the following investigation of this problem we include the consideration of the effect of gravity upon diffusion. Let us suppose that 
we have a shallow vessel whose volume is $z$ and that this is connected by a capillary tube of fine bore with another shallow vessel whose volume is $v^{\prime}$ situated at a height $h$ above the lower vessel. Let the two vessels be filled with water containing a certain quantity of salt dissolved in it, then we wish to find how the salt is divided between the vessels when equilibrium is established. Let $\xi$ and $\eta$ be the quantities of salt in the lower and upper vessels respectively, then if $h^{\prime}$ be the height of the lower vessel above some fixed plane, the potential energy of the salt in the lower vessel may be taken to be $\xi g h^{\prime}$, so that there is the term $-\xi_{g} h^{\prime}$ in the expression for the mean Lagrangian function of this salt, similarly there is the term $-\eta g\left(h+h^{\prime}\right)$ in the expression for the mean Lagrangian function of the salt dissolved in the upper vessel.

Thus using the same notation as before the expression for the mean Lagrangian function of the salt dissolved in the lower vessel is

$$
\xi R \theta \log \frac{\rho_{0} v}{\xi}+\xi f(\theta)-\xi w-\xi g h^{\prime} \ldots \ldots . .(188),
$$

the mean Lagrangian function of the salt dissolved in the upper vessel is

$$
\left.\eta R \theta \log \frac{\rho_{0} v^{\prime}}{\eta}+\eta f(\theta)-\eta w-\eta g\left(h^{\prime}+h\right) \ldots \text { (I } 89\right) \text {. }
$$

Let us suppose that a quantity $\delta \eta$ of salt goes from the lower to the upper vessel, then if there is equilibrium this change must not alter the value of $H$, the mean Lagrangian function of the salt and solvent in the two vessels. If the solutions are dilute the only part of $H$ which varies is the sum of the expressions (188) and (189), and the condition

$$
\frac{d H}{d \eta}=0
$$


leads to the equation

$$
R \theta \log \frac{v^{\prime} \xi}{v \eta}-g h=0,
$$

or if $\xi^{\prime}, \eta^{\prime}$ are the masses of salt in unit volume in the lower and upper vessels respectively

or

$$
\begin{gathered}
R \theta \log \frac{\xi^{\prime}}{\eta^{\prime}}-g h=0, \\
\frac{\eta^{\prime}}{\xi^{\prime}}=e^{-\frac{g h}{R \theta}} \ldots \ldots . .
\end{gathered}
$$

So that the concentration of the solution when there is equilibrium varies in the same way with the height as the density of a gas under the action of gravity.

97. A large number of experiments have been made on the effect of dissolved salts on the coefficients of compressibility of various solutions (see Schumann "Compressibilität von Chlorid Lösungen," Wied. Ann. xxxi. p. r4, 1887 and Röntgen and Schneider, Wied. Ann. xxix. p. I65, I886), we shall therefore investigate an expression for this effect and see what information can be gained by comparing it with the results of the above-mentioned experiments.

Let us suppose that the solution whose original volume is $v_{0}$ is subjected to a hydrostatic pressure $p$ which reduces its volume to $v$, and that $k^{\prime}$ is its coefficient of compressibility. Then the mean Lagrangian function of the solution and the system producing the pressure is

$$
-\frac{\mathrm{I}}{2 k^{\prime}} \frac{\left(v_{0}-v\right)^{2}}{v_{0}}+p\left(v_{0}-v\right)
$$

the mean Lagrangian function of the dissolved salt is, using the same notation as hitherto, 


$$
\xi R \theta \log \frac{v \rho_{0}}{\xi}+\xi f(\theta)-\xi z^{\prime}
$$

where $\xi$ is the mass of the salt.

If $H$ is the sum of these expressions then by the Hamiltonian principle $H$ must be stationary when there is equilibrium. Let us suppose that the volume is increased by $d \tau$, then since $H$ is stationary we must have

or

$$
\begin{gathered}
\frac{d H}{d v}=0, \\
\frac{\mathrm{I}}{k^{\prime}} \frac{\left(v_{0}-v^{\prime}\right)}{v_{0}}-p+\frac{\xi R \theta}{v}=0 \ldots . . . . . . \text { ( I 9 I); }
\end{gathered}
$$

now $\xi R \theta / 2$ is the pressure due to the molecules of the salt, let us call this $P$. If $p$ be increased by $\delta p$, the corresponding diminution in volume $\delta v$ is by (I9r) given by the equation

or

$$
\begin{aligned}
& \frac{\mathbf{I}}{\bar{k}^{\prime}} \frac{\delta v}{v_{0}}-\delta p+\frac{\xi R \theta}{v^{2}} \delta v=0, \\
& \delta p=\frac{\mathbf{I}}{k^{\prime}} \frac{\delta v}{v_{0}}\left\{\mathrm{I}+\frac{P k^{\prime} v_{0}}{v}\right\},
\end{aligned}
$$

or since $z_{0}$ is very nearly equal to $v$ we may write this equation in the form

$$
\delta p=\frac{\mathbf{I}}{k^{\prime}} \frac{\delta v}{v_{0}}\left\{\mathrm{I}+P k^{\prime}\right\} .
$$

So that the apparent coefficient of compressibility is

$$
\frac{k^{\prime}}{\mathbf{I}+P k^{\prime}} ;
$$

thus the pressure due to the molecules of the dissolved salt produces a decrease in the coefficient of compressibility. Let us see what the magnitude of this effect would be if the pressure of the molecules were the only way in which the dissolved salt affected the resistance to compression. If we make this assumption $k^{\prime}=1 / 2: 2 \times 10^{10}$, this being the 
value when measured in C. G. S. units of this constant for pure water at $15^{\circ} \mathrm{C}$. If there is one equivalent of salt in a litre of water, $P$ is 22 atmospheres or in absolute measure $2.2 \times 10^{7}$. Since the reduction in the coefficient of compressibility is very nearly equal to

$$
P k^{\prime 2} \text {, }
$$

or to one part in $I / P k^{\prime}$, we see that when the strength of solution is one equivalent per litre the reduction in the coefficient of compressibility ought to amount to one part in

$$
\frac{1}{2 \cdot 2 \times 10^{7}} \times 2 \cdot 2 \times 10^{10}
$$

that is to one part in rooo.

The following table taken from Röntgen's and Schneider's paper will show that the effect of dissolved salts is sometimes more than a hundredfold that calculated on the above assumptions, and hence we conclude that in addition to producing a pressure in the solvent the dissolved salt

\begin{tabular}{|c|c|c|}
\hline $\begin{array}{l}\text { Names of salt } \\
\text { or acid. }\end{array}$ & $\begin{array}{l}\text { Strength of } \\
\text { solution in } \\
\text { equivalents per } \\
\text { litre. }\end{array}$ & $\begin{array}{c}\text { Reduction in com- } \\
\text { pressibility found } \\
\text { by Röntgen and } \\
\text { Schneider reckuned } \\
\text { in parts } \\
\text { per thousand. }\end{array}$ \\
\hline $\mathrm{HNO}_{3}$ & I 49 & 42 \\
\hline $\mathrm{HBr}$ & I.49 & 40 \\
\hline $\mathrm{HCl}$ & $I^{\circ} 5^{2}$ & 52 \\
\hline $\mathrm{H}_{2} \mathrm{SO}_{4}$ & $r \cdot 48$ & 79 \\
\hline $\mathrm{NH}_{3} \mathrm{I}^{4}$ & $r \cdot 49$ & 90 \\
\hline $\mathrm{NH}_{3} \mathrm{NO}_{3}$ & $x \cdot 5$ & 94 \\
\hline $\mathrm{NH}_{3} \mathrm{Br}$ & $1 \cdot 5$ & 90 \\
\hline $\mathrm{NH}_{3} \mathrm{Cl}$ & I 45 & 97 \\
\hline $\mathrm{Na}_{2} \mathrm{CO}_{3}$ & I 5 & $37 x$ \\
\hline
\end{tabular}
must directly alter its elastic properties. 
98. The pressure due to the molecules of the dissolved salt will explain many of the phenomena exhibited by solutions. The molecules of the salt may be regarded as confined within a limited volume by the solvent, and they will take any opportunity of expanding even though they may have to do work to enable them to do so. Thus if the solution was contained in a vessel provided with a bottom pervious to water but impervious to the substance dissolved in it, then if the vessel is placed in water with its top above the surface water will flow up into the vessel through the bottom, the work required to lift the water being supplied by the expansion of the molecules of the dissolved salt. This constitutes the well-known phenomenon of osmosis.

A diaphragm which is said to be impervious to all salts though it allows water to pass through it can be made by allowing weak solutions of sulphate of copper and ferrocyanide of potassium to diffuse into a porous plate from opposite sides, these solutions when they meet form a membrane of the kind desired. Detailed instructions for making these membranes are given in Pfeffer's Osmotische Untersuchungen, Leipzig, 1877. By following his directions I have succeeded in making such membranes though the number of failures was very large compared with the number of successes. $\mathrm{Mr}$ Adie, who is making some investigations on this subject at the Cavendish Laboratory, finds that the membranes are formed more readily if ferric chloride is used instead of copper sulphate.

We shall now attempt to find by means of Hamilton's principle the height to which the fluid will rise in the osmometer. Let us suppose that the osmometer is a long tube with a diaphragm of the kind we have been describing at the bottom, and that it contains water and salt. Let $\xi$ 
be the mass of the salt, $\eta$ that of the water inside the tube, $\zeta$ that of the water outside, and let $v$ be the volume of the tube occupied by the solution. Then, using the same notation as hitherto, the mean Lagrangian function for the salt is

$$
R \theta \xi \log \frac{v \rho_{0}}{\xi}+\xi f(\theta)-\xi\left(w_{1}+g \bar{z}\right) \ldots \ldots(192),
$$

where $\bar{z}$ is the height of the centre of gravity of the salt molecules above some fixed plane.

The mean Lagrangian function for the liquid in the tube is

$$
\eta \gamma^{\prime} \theta+\eta f_{1}^{\prime}(\theta)-\eta\left(w_{2}^{\prime}+g \bar{z}\right) \ldots \ldots . .(\text { ( } 93),
$$

and for the liquid outside the tube

$$
\zeta \gamma \theta+\zeta f_{1}(\theta)-\zeta\left(w_{2}+g \bar{y}\right) \ldots \ldots \ldots \ldots(\text { I } 94),
$$

where $\bar{y}$ is the height of the centre of gravity of the water outside the osmometer : the quantities for the liquid inside the tube are denoted by affixing dashes to the symbols denoting the corresponding quantities for the water outside the tube.

By the Hamiltonian principle the value of $H$, the sum of (192), (193) and (194), is stationary when there is equilibrium. Let us suppose that a quantity of water $\delta \eta$ flows into the osmometer.

Then since if there is no contraction

$$
\frac{d v}{d \eta}=\mathrm{I}, \quad \frac{d \bar{z}}{d \eta}=\frac{\mathbf{I}}{2 a},
$$

where $\alpha$ is the area of the cross section of the osmometer, and

$$
\frac{d}{d \eta}(\eta g \bar{z}+\zeta g \bar{y})=g h,
$$


where $h$ is the height of the top of the fluid in the osmometer above the level of that outside : the condition

leads to the equation

$$
\frac{d H}{d \eta}=\text { o }
$$

$$
\begin{aligned}
\frac{R \theta \xi}{z^{\prime} \sigma}+ & \left(\gamma^{\prime}-\gamma\right) \theta+f_{1}^{\prime}(\theta)-f_{1}(\theta)+\eta \frac{d \gamma^{\prime}}{d \eta} \theta \\
& +\eta \frac{d f_{1}^{\prime}(\theta)}{d \eta}-\frac{d}{d \eta}\left(\eta \iota_{2}^{\prime}\right)+z \ell_{2}^{\prime}-g\left(h+\frac{\xi}{2 \alpha}\right)=0 \ldots(195)
\end{aligned}
$$

if the properties of the solution are not altered by the presence of the salt then

$$
\gamma^{\prime}=\gamma, f_{1}^{\prime}(\theta)=f_{1}(\theta), z v_{2}^{\prime}=\tau c_{2}^{\prime}
$$

and equation (195) becomes

$$
p=g \sigma\left(h+\frac{1}{2} h^{\prime}\right),
$$

where $p$ is the pressure due to the molecules of the dissolved salt, and $h^{\prime}$ the height of a column of water whose mass is the same as that of the salt dissolved in the osmometer. If the strength of the solution in the osmometer is one equivalent per litre, $p$ is about 22 atmospheres, so that in this case $h+\frac{1}{2} h^{\prime}$ is about 660 feet; that is the water would flow into the osmometer until the height of the liquid in the tube is nearly an eighth of a mile above the level of the water outside.

If the liquid is not allowed to expand but confined in a constant volume we can easily prove in a similar way that if the properties of the solvent are not changed by the addition of the salt then when there is equilibrium the pressure exerted by the fluid in the osmometer must be the same as that due to the molecules of the salt. This result is given by Van t' Hoff (L'équilibre chimique, Archiz' Neerlandais t. 20, p. 239).

Pfeffer (Osmotische Untersuchungen, p. 12) gives as the 
pressure for a $1 \%$ solution of potassium sulphate that due to 192. 6 centimetres of mercury and for a $\mathrm{r} \%$ solution of potassium nitrate that due to 178.4 centinietres. The pressure calculated on the above principles for potassium sulphate is 97 centimetres if we assume that the molecule is $\mathrm{K}_{8} \mathrm{SO}_{4}$ and 194 if the molecule is $\frac{1}{2}\left(\mathrm{~K}_{2} \mathrm{SO}_{4}\right)$, for potassium nitrate it is 167 if the molecule is $\mathrm{KNO}_{3}$.

We see as in $\$ 90$ that the terms in (195) depending upon the alteration of the properties of the solvent by the addition of the salt do not contain any powers of the strength of the solution below the second.

A measurement of the osmotic pressure produced by any salt solution will on the above assumptions give the same information about the structure of the molecule of the salt in the solution as a vapour density determination does about the structure of the gas whose vapour density is determined, for it enables us to find the number of molecules in a given mass of the gas. Thus Pfeffer's measurement of the osmotic pressure due to potassium sulphate suggests that the relation between the composition of the molecule of this salt and that of potassium nitrate is represented by $\frac{1}{2} \mathrm{~K}_{9} \mathrm{SO}_{4}$ and $\mathrm{KNO}_{3}$, and not by $\mathrm{K}_{2} \mathrm{SO}_{4}$ and $\mathrm{KNO}_{3}$.

Even if we do not assume that the molecules of a salt produce a pressure analogous to that of a gas, it would still follow from the Hamiltonian principle that there would be a rise in the osmometer if the increase in the mean Lagrangian function of the liquid inside the osmometer caused by the addition of unit mass of water is greater than the diminution in the mean Lagrangian function in the water outside the osmometer caused by the abstraction of unit mass of water.

Anything that causes a change of this kind will increase the height to which the fluid will rise in the osmometer; 
thus, if the addition of water to the solution inside the osmometer is attended by an evolution of heat, the solution will rise higher in the osmometer than one of similar strength in which no heat was evolved in dilution. On this account the indications of the osmometer are somewhat ambiguous, and before coming to any definite conclusion as to the structure of the molecule of the salt it would be necessary to use several solvents and to show that the osmotic height varied as the absolute temperature.

99. Surface Tension of Solutions. The experiments of Röntgen and Schneider already alluded to have proved that for most solutions the product of the height to which the solution rises in a capillary tube into the density of the solution is greater for a solution of a salt than for pure water, and that for dilute solutions of most (though not all) substances this product increases with the strength of the solution. It follows from this that the tension of the surface of contact of the solution with air increases with the strength of the solution, while the tension of the surface of contact of the solution with glass or any other solid body diminishes as the solution gets stronger.

The variation of the surface tension with the strength of solution may cause the strength of the solution to vary near the surface.

To investigate the magnitude of this effect let us suppose that we have a thin film whose area is $S$ and surface tension $T$, connected with the bulk of the liquid by a capillary thread. Let $\xi$ be the mass of salt in the thin film, $\eta$ that in the rest of the liquid; then if $\zeta$ is the mass of water in the film, $\epsilon$ that of the rest of the water, the mean Lagrangian function of the liquid and salt in the film is, using the same notation as before, 


$$
\xi R \theta \log \frac{v \rho_{0}}{\xi}+\xi f(\theta)-\xi w_{1}+\zeta \gamma \theta+\zeta f_{1}(\theta)-\zeta w_{2}-S T .
$$

where $v$ is the volume of the film.

The mean Lagrangian function for the rest of the liquid is

$$
\eta R \theta \log \frac{v^{\prime} \rho_{0}}{\eta}+\eta f(\theta)-\eta w_{1}+\epsilon \gamma \theta+\epsilon f_{1}(\theta)-\epsilon \tau \mho_{2} .
$$

Let us suppose that a mass of salt $\delta \xi$ goes into the film, the change in the mean Lagrangian function is

$$
\left(R \theta \log \frac{v}{\xi} \frac{\eta}{v^{\prime}}-S \frac{d T}{d \xi}\right) \delta \xi,
$$

and this by the Hamiltonian principle must vanish; thus if $\rho, \rho^{\prime}$ are the masses of salt in unit volume of the film and liquid respectively, we get

$$
\frac{\rho^{\prime}}{\rho}=\epsilon^{\frac{S}{R \theta}} \frac{d T}{d \xi},
$$

or if $T^{\prime}$ is the increase in the surface tension when the mass of salt in unit volume is increased by unity

$$
\frac{\rho^{\prime}}{\rho}=\epsilon^{\frac{2}{R} T^{\prime}}
$$

where $t$ is the thickness of the film. Thus if the surface tension is increased by the addition of the salt there will be less salt per unit volume in the film than in the liquid in bulk, while if the surface tension is diminished by the addition of salt there will be more salt in unit volume of the film than in unit volume of the rest of the liquid. IVe saw that the surface tension of a solution in contact with a solid diminished as the strength of the solution increased, thus if we had a film in contact with a solid there would be more salt in unit volume of the film than in unit volume of the bulk of 
the liquid; if we dipped for example a piece of filter paper in such a solution, the solution in the filter paper would be stronger than the rest. Or, again, if such a solution were to flow through a capillary tube the salt would have a tendency to flow to the sides, so that the more quickly moving fluid at the centre would get weaker and weaker. Many experimental illustrations of this could be given; one of these is an experiment tried by Dr Monckman and myself at the Cavendish Laboratory, in which a deep coloured solution of potassium permanganate emerged almost colourless after trickling through finely divided silica. Again, if a piece of filter paper be dipped into a coloured solution of a salt such as potassium permanganate, unless the salt has a very strong affinity for the water the solution after rising some height in the filter paper becomes colourless.

If a small quantity of paraffin oil be mixed with water the surface tension of the solution against a solid is greater than that of water, and such a solution will increase in strength when it flows through finely divided silica. 


\section{CHAPTER XIII.}

\section{DISSOCIATION.}

I 00. THE Hamiltonian method can be used for the purpose of obtaining the laws which govern the phenomena of dissociation, i.e. the splitting up of a molecule into its atoms, such as the iodine molecule $I_{2}$ into the atoms $I$ and $I$; or of a complex molecule into simpler ones, as in the case of nitrogen tetroxide, where the molecule $\mathrm{N}_{2} \mathrm{O}_{4}$ splits up into two molecules of $\mathrm{NO}_{2}$, or when the molecule of chloride of ammonium splits up into ammonia and hydrochloric acid.

This phenomenon has some analogy with that of evaporation; as in the latter case we have equilibrium between portions of matter in two different states, the gaseous and the liquid, matter being able to pass from the one state to the other by evaporation and condensation, so in dissociation we have also equilibrium between portions of the same substance in two different conditions, both in the gaseous state, the molecules in the one condition being more complex than those in the other, and matter being able to pass from one condition into the other by the more complex molecules splitting up, "dissociating" as it is called into the simpler ones, while on the other hand some of the simpler ones combine and form the more complex molecules. Equilibrium is attained when the number of the complex

T. D. 
molecules which split up in any time is the same as the number formerl in the same time.

Let us first investigate the case when the complex molecules contain two of the simpler ones; this is the case when, as in iodine, the more complex systems are di-atomic molecules and the simpler ones atoms, as well as in such cases as the dissociation of $\mathrm{N}_{8} \mathrm{O}_{4}$.

Let us suppose that the system is contained in a closed vessel and that $\xi$ is the mass of the complex molecules, $\eta$ that of the simpler ones. We shall for the present assume that both gases obey Boyle's law and that the fundamental equation for the complex gas is

and for the simpler gas

$$
p=R_{1} \rho \theta,
$$

$$
p=R_{\mathrm{g}} \rho \theta
$$

where $p$ is the pressure, $\rho$ the density and $\theta$ the absolute temperature.

Since the molecules of the complex gas consist of two of those of the simpler gas, the density of the simpler gas will at the same pressure and temperature be half that of the complex gas and therefore

$$
R_{2}=2 R_{1} \text {. }
$$

The mean Lagrangian function of the complex gas is

$$
\xi R_{1} \theta \log \frac{v \rho_{0}}{\xi}+\xi f_{1}(\theta)-\xi z \ell_{1} \ldots \ldots \ldots(\mathrm{I} 96),
$$

where $v$ is the volume of the vessel in which the gas is contained and $w_{1}$ the potential energy of unit mass of the complex gas.

The mean Lagrangian function for the simpler gas is

$$
\left.\eta R_{\mathrm{z}} \theta \log \frac{v \rho_{0}^{\prime}}{\eta}+\eta f_{\mathbf{z}}(\theta)-\eta w_{\mathrm{a}} \ldots \ldots \ldots \text {.... (1 } 97\right),
$$

where $w_{z}$ is the potential energy of unit mass of this gas. 
The mean Lagrangian function $H$ for the two gases, assuming that the properties of each are not modified by the presence of the other, is the sum of the expressions (196) and (197). By the Hamiltonian principle the value of $H$ is stationary when the system is in equilibrium. Let us suppose that the state of equilibrium is disturbed by a mass $\delta \xi$ of the simpler molecules combining to form complex ones. Then since the value of $H$ is stationary we must have

$$
\frac{d H}{d \xi}=0 .
$$

Since the mass of the gas is constant

$$
\delta \xi+\delta \eta=0
$$

and the condition

$$
\frac{d H}{d \xi}=0
$$

is equivalent to

$$
\begin{aligned}
& \begin{aligned}
R_{1} \theta \log \frac{v \rho_{0}}{\xi}-R_{1} \theta+f_{1}(\theta) & -w_{1}-R_{2} \theta \log \frac{v \rho_{0}^{\prime}}{\eta} \\
& +R_{2} \theta-f_{2}(\theta)+w_{2}=0 \ldots \ldots(\mathrm{I} 98), \\
\text { or since } & R_{2}=2 R_{1}
\end{aligned}
\end{aligned}
$$

we have

$$
R_{1} \theta \log \frac{\eta^{2} \rho_{0}}{\xi v \rho_{0}^{\prime 2}}+R_{1} \theta+f_{1}(\theta)-f_{2}(\theta)=w_{1}-w_{2} .
$$

This can be written

$$
\frac{\eta^{2}}{v \xi}=\phi(\theta) \epsilon^{\frac{w_{1}-w_{2}}{R_{1} \theta}}
$$

where $\phi(\theta)$ is a function of $\theta$ but not of $\xi, \eta$ or $v$.

In experiments on dissociation the quantity usually measured is the vapour density of the mixture at some standard pressure $\pi$. 
Let $\Delta$ be the density of the mixture of the two gases at this pressure, $D$ that of the complex or undissociated gas at the same pressure.

The pressure in the vessel is

$$
\frac{\xi R_{1} \theta}{v^{\prime}}+\frac{\eta R_{2} \theta}{v^{\prime}}
$$

or since

$$
R_{2}=2 R_{1}
$$

the pressure equals $\frac{\xi+2 \eta}{v} R_{1} \theta$.

The density of the gas at this pressure is

$$
\frac{\xi+\eta}{v}
$$

so that $\Delta$ the density at the pressure $\pi$ is given by the equation

$$
\Delta=\frac{\xi+\eta}{\xi+2 \eta} \frac{\pi}{R_{1} \theta}
$$

the density $D$ of the complex gas at this pressure is given by the equation

so that $\Delta=\frac{\xi+\eta}{\xi+2 \eta} D$,

$$
D=\frac{\pi}{R_{1} \theta},
$$

and therefore

$$
\frac{\eta}{\xi+2 \eta}=\frac{D-\Delta}{D}
$$

and

$$
\frac{\xi}{\xi+2 \eta}=\frac{2 \Delta-D}{D} \text {; }
$$

since

$$
p^{\prime \prime}=(\xi+2 \eta) R_{1} \theta \text {, }
$$

we have

$$
\begin{gathered}
\eta=\frac{p v}{R_{1} \theta} \frac{D-\Delta}{D}, \\
\xi=\frac{p v}{R_{1} \theta} \frac{2 \Delta-D}{D},
\end{gathered}
$$


so that equation (199) becomes

$$
\frac{p(D-\Delta)^{2}}{D(2 \Delta-D)}=\phi_{1}(\theta) \epsilon^{\frac{w_{1}-w_{2}}{R_{1} \theta}} \ldots \ldots \ldots \ldots(200),
$$

where

$$
\phi_{1}(\theta)=R_{1} \theta \phi(\theta) .
$$

гог. Before discussing this equation we shall investigate the way in which it must be modified if the gas does not obey Boyle's law.

Formulae connecting the pressure and volume in such gases have been given by Van der Waals (Die Continuität des gasformigen una flïssigen Zustandes) and Clausius (Wied. Ann. 1X. p. 337).

Van der Waals' formula, which is rather the simpler of the two, is

$$
p=\frac{R \theta}{v-b}-\frac{a}{v^{2}},
$$

where $R$ is the value of $p / \rho \theta$ for a perfect gas of the same specific gravity, and $b$ and $a$ constants depending upon the nature of the gas.

Clausius' formula is

$$
p=\frac{R \theta}{v-\alpha}-\theta \frac{\kappa}{\theta(\bar{v}+\beta)^{2}},
$$

where $R$ is the same as in Van der Waals' formula for the same gas, and $\alpha, \beta, \kappa$ are small constants depending upon the nature of the gas. We shall now investigate the difference produced in the state of equilibrium of a dissociable gas if it and the components into which it is decomposed obey Van der Waals' law instead of Boyle's. 
Let the fundamental equations of the complex and simple gases be respectively

$$
p=\frac{R_{1} \theta}{v-b_{1}}-\frac{a_{1}}{v^{2}}
$$

and

$$
p=\frac{R_{2} \theta}{v^{\prime}-b_{2}}-\frac{a_{9}}{v^{2}}
$$

where

as before.

$$
R_{2}=2 R_{1}
$$

'Then we can easily prove that instead of the term

$$
\xi R_{1} \theta \log \frac{\rho_{0}}{\rho}
$$

in the mean Lagrangian function, we have the term

$$
\xi R_{1} \theta \log \left(\frac{\mathrm{I}-b_{1} \rho}{\rho} \rho_{0}\right)+\xi a_{1} \rho,
$$

where $\rho$ is the density of the complex gas; with a corresponding term in the expression for the Lagrangian function of the simpler gas.

The condition

$$
\frac{d H}{d \xi}=0
$$

will now lead to the equation

$$
\begin{aligned}
R_{1} \theta\left\{\log \left(\mathrm{I}-b_{1} \rho\right)+\log \frac{\rho_{0}}{\rho}-\frac{\mathbf{I}}{\mathbf{I}-b_{1} \rho}\right\} \\
-R_{2} \theta\left\{\log \left(\mathbf{I}-b_{2} \rho^{\prime}\right)+\log \frac{\rho_{0}^{\prime}}{\rho^{\prime}}-\frac{\mathbf{I}}{\mathbf{I}-b_{2} \rho^{\prime}}\right\}+2 a_{1} \rho-2 a_{2} \rho^{\prime} \\
\quad+f_{1}(\theta)-f_{2}(\theta)-\left(w_{1}-w_{2}\right)=0 \ldots \ldots \ldots . .(2 \circ \mathrm{I})
\end{aligned}
$$

and not to the equation $(198)$. 
Equation (20I) may be written, since $R_{2}=2 R_{1}$,

$$
\begin{aligned}
R_{1} \theta\left\{\log \frac{\eta^{g}}{v \xi}+\log \frac{\mathbf{I}-b_{1} \frac{\xi}{v}}{\left(1-b_{2} \frac{\eta}{v}\right)^{2}}\right. & \left.+\log \frac{\rho_{0}}{\rho_{0}^{\prime g}}-\frac{\mathbf{I}}{\mathbf{I}-b_{1} \frac{\xi}{v \prime}}+\frac{2}{\mathbf{I}-b_{2} \frac{\eta}{v}}\right\} \\
& +\frac{2\left(a_{1} \xi-a_{2} \eta\right)}{v}+f_{1}(\theta)-f_{2}(\theta) \\
= & w w_{1}-w_{2}^{\prime} .
\end{aligned}
$$

Now if we suppose that the deviations from Boyle's Law are slight, so that $b_{1}$ and $b_{2}$ are so small that their squares may be neglected, we may write this equation as

$$
\frac{\eta^{2}}{v \xi}=\phi(\theta) \epsilon^{-\frac{2\left(a_{1}-b_{1}\right) \xi-2\left(a_{2}-2 b_{2}\right) \eta}{v R \theta}} \epsilon \frac{\left(z v_{1}-z v_{2}\right)}{R_{1} \theta} \ldots \ldots .(2 \circ 2) ;
$$

since $\xi / v R_{1} \theta$ is approximately equal to $\rho^{2} / p$ and $a_{1}$ and $\rho$ are both small fractions while $p=10^{6}$ if the pressure is one atmosphere, equation (202) may be written as

$$
\frac{\eta^{2}}{v \xi}=\phi(\theta) \epsilon^{\frac{\left(z v_{1}-z v_{2}\right)}{R \theta}},
$$

an equation of the same form as when the gases obeyed Boyle's law. The connexion between the masses of the complex and simple gases and the vapour density of the mixture will not however be the same as when the gases obeyed Boyle's law, and so the relation between the vapour density, the pressure and the temperature may be different although equation (202) shows that the relation between the masses of the dissociated and undissociated gases is the same.

It would be an interesting problem to find an expression for the vapour density of the mixture in terms of the masses of the two gases in this case, we shall not however stop to 
investigate it as it would not be of any use for the purpose of connecting theory with experiment, for in determining the vapour density from the experiments Boyle's law was no doubt assumed.

I02. Formulae corresponding to equation (200) deduced from thermodynamical considerations have been given by Willard Gibbs (Equilibrium of Heterogeneous Substances, p. 239) and Boltzmann (Wied. Ann. xxir. p. 39, r 884).

Thus according to Gibbs

$$
\frac{p(D-\Delta)^{2}}{D(2 \Delta-D)}=C e^{-\frac{\alpha}{\theta}} \ldots \ldots \ldots \ldots . . . .(2 \circ 3),
$$

this agrees with (200) if $\phi_{1}(\theta)$ is constant.

According to Boltzmann

$$
\frac{p(D-\Delta)^{2}}{D(2 \Delta-D)}=C \theta e^{-\frac{\alpha}{\theta}} \ldots \ldots \ldots \ldots . . .(204),
$$

and this agrees with $(200)$ if $\phi_{1}(\theta)$ is proportional to $\theta$.

Gibbs in his paper ("On the vapour densities of peroxide of nitrogen, formic acid, acetic acid and perchloride of phosphorus," American Journal of Science and Art, xvill. p. 277,1879 ), discusses the results of experiments on the vapour densities of these substances at different temperatures and pressures and has found that they agree fairly well with the results calculated by formula $(2 \circ 3)$. Quite recently however E. and L. Natanson have made a most elaborate investigation of the vapour density of nitrogen tetroxide at various temperatures and pressures (Wied. Ann. xxvil. p. 306). They found that so long as the temperature remains constant the vapour density of nitrogen tetroxide at different pressures is given with great accuracy by the formula $(200)$ but that if the 
temperature changes the difference between the observed results and those calculated from either Gibbs' or Boltzmann's formula, assuming that the quantity $\alpha$ which occurs in it is constant, is greater than can be accounted for by errors of experiment. Part of this difference may arise because the $\mathrm{N}_{8} \mathrm{O}_{4}$ does not obey Boyle's law. The differences seem however to be too great to be explained altogether in this way, and a value of $\phi_{1}(\theta)$ different from that adopted by either Gibbs or Bolt $\iota m a n n$ would probably fit in better with the observations.

ro3. In the Philosophical Magazine for October, $188_{4}$, I considered the question of dissociation from the point of view of the kinetic theory of gases, supposing that the complex molecules are continually being broken up while the simpler ones are continually combining, and that the gas attains a steady state when the number of complex molecules broken up in the unit time is the same as the number formed in that time. It is shown that, using the same notation as in $\$ 99$, these conditions lead to the equation

$$
\frac{\eta^{2}}{\eta \xi}=f(\theta)
$$

if, and only if, the average time a complex molecule lasts without splitting up into simpler ones, is independent of the number of molecules of the gas in unit volume. This will evidently not be the case if the breaking up of the complex molecules is due to their collision with other molecules, for in this case, the greater the number of molecules the greater the number of collisions, and therefore the shorter the time the complex molecule lasts. Since the results of a large number of experiments prove that equation (200) holds when the temperature is constant we conclude that the dissociation of 
the complex molecules is not due to the collision with other molecules. We have however deduced (200) from mechanical principles which hold whenever the two gases obey Avogadro's law and whenever the pressure produced by a mixture of gases is the sum of the pressures which would be produced by each of the gases separately if the other were removed. Hence we conclude that when we have a gas some of whose molecules are complex and keep breaking up into simpler molecules which after a time recombine to form the complex molecules, then if the splitting up of the complex molecules is due to their striking against other molecules, the pressure due to the gas will not be the sum of the pressures which the dissociated and undissociated gases would produce if each were by itself in the vessel.

104. We shall now consider how external influences may modify the amount of dissociation which takes place in some given gas at a given temperature and pressure.

If we denote $\eta^{2} / v \xi$ by $\lambda$ and use $\lambda$ as a measure of the amount of dissociation, then if the Lagrangian function from some external cause is increased by $\chi$ we see by equation (198) that $\delta \lambda$ the change in $\lambda$ is given by the equation

$$
R_{1} \theta \frac{\delta \lambda}{\lambda}+\frac{d \chi}{d \xi}=0 . \ldots \ldots \ldots \ldots(205) \text {. }
$$

Thus if $\chi$ increases as $\xi$ diminishes-that is as dissociation goes on-- $\delta \lambda$ will be positive, that is dissociation will go on further than it did in the undisturbed state. This is another illustration of the general principle stated in $\$ 84-$ that any slight alteration in the conditions under which a system is placed which increases the rate of increase of the mean Lagrangian function with any change in the system, will cause that change to go on further before equilibrium is. 
attained than it had to do in the undisturbed system and vice versâ.

We shall now consider the effects on dissociation of such things as surface tension, electrification, the presence of other gases, corresponding to those we considered in the analogous case of evaporation.

105. Effect of surface tension upon dissociation. Though the effects of surface tension are not nearly so prominent in gases as in liquids, still, since there is perfect continuity from the liquid to the gaseous state, we should expect that the outer layer of molecules of a gas which was not in the "perfect" condition would like the outer layer in a liquid be under different conditions from the other molecules, and would therefore not possess the same amount of energy as the same number of molecules in the midst of the gas.

In Van der Waals' theory of the relation between the pressure and volume in an imperfect gas, the result of which is expressed by the relation

$$
\left(p+\frac{a}{v^{2}}\right)(v-b)=R \theta,
$$

the term $a / z^{2}$ is due to the action of the surface tension of the gas (Van der Waals, Die Continuität des gasformigen und flüssigen Zustands, p. 34).

Though it is much more difficult to detect the existence of the action of surface tension experimentally in gases than in liquids there is still some evidence of its existence from experiments such as those of Bosscha on the forms of clouds of fog and tobacco smoke.

There must therefore be a term in the expression for the potential energy of a gas proportional to its surface. IVe shall write this term 
where $T$ is the quantity corresponding to the surface tension and $S$ is the area of the surface of the gas. Thus the change $x$ in the Lagrangian function, $\S 104$, is

$$
\text { - } T S \text {. }
$$

so that by (205)

$$
R_{1} \theta \frac{\delta \lambda}{\lambda}-\frac{d}{d \xi}(T S)=0 \ldots \ldots \ldots \ldots(206) .
$$

Thus, if the surface tension diminishes as dissociation goes on, in which case $d T / d \xi$ is positive, the dissociation will be greater the larger the surface of the gas. We should expect $a$ priori that the surface tension of the dissociated gas would be smaller than that of the undissociated, for in most cases the dissociated gas approaches more nearly than the other to the state of a perfect gas : thus in most cases $d T / d \xi$ will be positive, so that dissociation will be facilitated by increasing the surface of the gas.

Let us now endeavour to form a rough estimate of the magnitude of this effect. According to Van der Waals the energy of unit area of surface of gas is measured by

$$
\frac{x a}{v^{2}}
$$

where $x$ is a distance comparable with molecular distances. Now for a cubic centimetre of ether vapour at $0^{\circ} \mathrm{C}$. and under atmospheric pressure $a / v^{2}$ is a pressure of about $3^{24} \times 10^{-4}$ atmospheres, or in absolute measure $3^{.24} \times 10^{4}$. If we take the molecular distance $x$ as $10^{-7}$, we have

$$
\begin{aligned}
T=\frac{x a}{v^{2}} & =10^{-7} \times 3.24 \times 10^{4} \\
& =3.24 \times 10^{-3} .
\end{aligned}
$$


Now by equation (206)

$$
\frac{\delta \lambda}{\lambda}=\frac{I}{R_{1} \theta} \frac{d}{d \xi}(S T) .
$$

In order to form a rough estimate of the value of $d T / d \xi$, let us suppose that the complex gas possesses surface tension but that the simpler one does not; this is an approach to the truth, as the value of $a$ and therefore of the surface tension is very much greater for complex gases than for simple ones. Let $\rho$ be the density of the complex gas, $v$ the volume in which it is contained, then

$$
\xi=v \rho .
$$

Since the surface tension varies as $a / v^{2}$, it is proportional to the square of the density, so that

$$
\begin{aligned}
\frac{\mathbf{I}}{T} \frac{d T}{d \xi} & =\frac{2}{\rho} \frac{d \rho}{d \xi} \\
& =\frac{2}{v \rho},
\end{aligned}
$$

and thus

$$
\frac{d T}{d \xi}=\frac{2 T}{v \rho},
$$

so that we have

$$
\frac{\delta \lambda}{\lambda}=\frac{2}{R_{1} \theta_{\rho}} \frac{S T}{v} \ldots \ldots \ldots \ldots \ldots(207) .
$$

Now at the atmospheric pressure, at which we reckoned $T$,

$$
R_{1} \theta_{\rho}=10^{6},
$$

and substituting for $T$ its value, we have

$$
\frac{\delta \lambda}{\lambda}=\frac{2 S}{v} \frac{3}{10^{\circ}} \text { approximately. }
$$


If the gas be supposed to be a film of thickness $t$, then

$$
\frac{\delta \lambda}{\lambda}=\frac{1.2}{t \times 10^{8}}
$$

so that if the thickness of the film were comparable with molecular dimension, say if $t=10^{-7}$, then the surface tension would produce very large effects.

This example may be sufficient to show that if we have the gas in thin films surface tension may produce a very considerable effect; such films occur adhering to glass fibres or to matter in a fine state of division, such as spongy platinum or charcoal. The value of $T$ given above is only part of the surface tension of the surface of contact of the gas and the solid. The surface tension of the surfaces separating $A$ and $B$ is due to the energy of thin layers of $A$ and $B$ next their junction differing by a finite amount from the energy possessed by equally thin layers in their interior. The abnormal energy of these layers is due to the want of symmetry of the action on the two sides. In the preceding investigation we have calculated the part of the energy of the layer of one of these substances arising from the effects produced by its own molecules, in addition to this there is the energy arising from the action of the glass on the gas as well as the energy in the thin film of glass. Thus the value of the surface tension may be much greater than that given above and the effects due to it may therefore be greater than our estimate.

ro6. The value of $T$ may depend upon the substance to which the film adheres, and thus the nature of the walls of vessels used for chemical experiments may affect the chemical combination which goes on inside them. Van ' $t$ Hoff has described some experiments which seem to show that effects of this kind do exist. He shows (Études de Dynamique Chimique, p. 56) that the rate at which the 
polymerization of cyanic acid goes on is increased by increasing the area of the walls of the vessel in which it is contained, the volume being kept constant. Thus when the area of the walls was increased six times, the rate of polymerization was increased in the ratio of 4 to 3 . He also found that when the walls of the vessel were covered with a deposit of cyamelide the rate of polymerization of cyanic acid was increased threefold. Victor Meyer too found that the decomposition of carbonic acid takes place in a porcelain vessel at a temperature several hundred degrees lower than in a platinum vessel. When the effects produced are of this magnitude, it is doubtful whether they can be due to the effect of surface tension, but it is probable that in the case of many catalytic actions, where we have thin films of gas, the effects observed might be explained by considerations of this kind.

\section{Effect of Electricity upon Dissociation.} When there is no electric discharge electrification will not produce any effect upon the final state of the system, unless the specific inductive capacity of the gas changes as dissociation goes on. As all the specific inductive capacities of gases which have been determined are very nearly equal, the effect of electrification on dissociation must be very small, and we shall not stop to determine it.

108. Effect of a neutral gas. If the properties of the neutral gas are not affected in any way by the presence of the gas which is dissociating, the value of the mean Lagrangian function of the neutral gas will not change as dissociation goes on. The presence of this gas will therefore not affect the maximum amount of dissociation. The presence of a foreign gas certainly alters the rate of dissociation, and 
in some cases the experiments seem to show that it does alter the maximum amount of dissociation. This is contrary to the result we have just arrived at, and the only way of reconciling the two is to suppose that the gas is not perfectly neutra! but has its properties affected to some extent by the presence of the other gases. If the dissociation were at all catalytic, we might explain the action of the neutral gas by supposing that by itself forming a film on the surface of the vessel it prevented to some extent the dissociating gas from doing so.

109. In the preceding investigations we have assumed that the complex molecule splits up into two molecules or atoms of the same kind. In some cases however the constituents into which the molecule splits up are different, as for example when $\mathrm{PCl}_{5}$ splits up into $\mathrm{PCl}_{3}$ and $\mathrm{Cl}_{2}$.

We can easily modify the preceding investigation to suit cases of this kind.

Let us take the dissociation of phosphorus pentachloride as a typical case, and let $\xi, \eta, \zeta$ be the masses of $\mathrm{PCl}_{5}, \mathrm{PCl}_{3}$, and $\mathrm{Cl}_{2}$ respectively.

- Then the mean Lagrangian functions for these gases are respectively

$$
\begin{gathered}
\xi R_{1} \theta \log \frac{v \rho_{0}}{\xi}+\xi f_{1}(\theta)-\xi w_{1}, \\
\eta R_{2} \theta \log \frac{v \rho_{0}^{\prime}}{\eta}+\eta f_{2}(\theta)-\eta w_{2}, \\
\zeta R_{3} \theta \log \frac{\rho_{0}^{\prime \prime}}{\zeta}+\zeta f_{3}(\theta)-\zeta w_{3} .
\end{gathered}
$$

Now if $c_{1}, c_{2}, c_{3}$ are the molecular weights of these gases respectively, then since the increase in the number of mole- 
cules of $\mathrm{PCl}_{3}$ is the same as that in the number of $\mathrm{Cl}_{2}$ and to the decrease in the number of $\mathrm{PCl}_{5}$, we have

$$
\frac{d \xi}{c_{1}}=-\frac{d \eta}{c_{2}}=-\frac{d \zeta}{c_{3}}
$$

where $d \xi, d \eta, d \zeta$ are the alterations in the masses of $\mathrm{PCl}_{b}$, $\mathrm{PCl}_{3}$, and $\mathrm{Cl}_{2}$ respectively; hence, remembering that

$$
c_{1} R_{1}=c_{2} R_{2}=c_{3} R_{3} \text {, }
$$

we see that the condition

$$
\frac{d H}{d \xi}=0
$$

leads to the equation

$$
\frac{\eta \zeta}{\xi v}=\phi_{1}(\theta) \epsilon \frac{\left(w_{1}-\frac{c_{2}}{c_{1}} w_{2}-\frac{c_{3}}{c_{1}} w_{3}\right)}{R_{1}{ }^{\theta}} \ldots \ldots \ldots \ldots(2 \circ 8),
$$

and thus $\eta \zeta / \xi v$ is constant as long as the temperature is constani.

Let us suppose that the values of $\xi, \eta, \zeta$ before dissociation commenced were $\xi_{0}, \eta_{0}, \zeta_{0}$ and that the mass $c_{1} p$ of $\mathrm{PCl}_{5}$ gets decomposed, then we have

$$
\begin{aligned}
& \xi=\xi_{0}-c_{1} p, \\
& \eta=\eta_{0}+c_{2} p, \\
& \zeta=\zeta_{0}+c_{3} p,
\end{aligned}
$$

and the equation to find $p$ is

$$
\left(\eta_{0}+c_{2} p\right)\left(\zeta_{0}+c_{3} p\right)=k v\left(\xi_{0}-c_{1} p\right) \ldots \ldots(2 \circ 9)
$$

where $k$ is a function of the temperature.

We shall now discuss the effect upon $p$ of alterations in the values of $v, \eta_{0}$ and $\zeta_{0}$.

Differentiating (209) we get, writing $\gamma$ for

$$
\frac{c_{1}}{\xi_{0}-c_{1} p}+\frac{c_{2}}{\eta_{0}+c_{2} p}+\frac{c_{3}}{\zeta_{0}+c_{3} p},
$$

T. D. 


$$
\begin{aligned}
& \gamma \frac{d p}{d v}=\frac{\mathbf{I}}{v} \ldots \ldots \ldots \ldots \ldots \ldots \ldots(2 \mathbf{1 0}), \\
& \gamma \frac{d p}{d \eta_{0}}=-\frac{1}{\eta_{0}+c_{2} p} \ldots \ldots \ldots \ldots \ldots(2 \text { I I }), \\
& \gamma \frac{d p}{d \zeta_{0}}=-\frac{\mathbf{I}}{\zeta_{0}+c_{3} p} \cdots \cdots \ldots \ldots \ldots(2 \mathrm{2} 2) .
\end{aligned}
$$

We see from (210) that $d p / d v$ is positive, so that dissociation will be promoted by increasing the volume in which a given quantity of gas is confined. From equations (2II) and (2I2) we see that both $d p / d \eta_{0}$ and $d p / d \zeta_{0}$ are negative, so that the presence of free $\mathrm{PCl}_{3}$ and $\mathrm{Cl}_{2}$ tends to stop the dissociation. Würtz proved experimentally that there was very little dissociation of $\mathrm{PCl}_{5}$ when it was placed in an atmosphere of $\mathrm{PCl}_{3}$. We can also see from general principles that this must be so, for as soon as the molecule $\mathrm{PCl}_{5}$ breaks up the free chlorine will be surrounded by such a multitude of molecules of $\mathrm{PCl}_{3}$ that most of it will recombine and form $\mathrm{PCl}_{5}$, and in this way stop the dissociation.

In this case, as in the former, theory indicates that if there is no catalytic action the presence of a neutral gas would not produce any effect.

In some cases, though the results of the dissociation are in the gaseous state, the body which dissociates is in the solid or liquid state instead of, as in the previous instances, the gaseous. The dissociation of $\mathrm{NH}_{5} \mathrm{~S}$ into $\mathrm{H}_{2} \mathrm{~S}$ and $\mathrm{NH}_{3}$ is an example of this kind.

We have only to slightly modify the preceding work to make it applicable to this case. Let as before $\xi$ be the mass of the dissociating body, $\eta$ and $\zeta$ those of the components into which it is dissociated. Then the mean Lagrangian function for the solid or liquid dissociating body is by $\S(8 \mathbf{r})$

$$
\xi \gamma \theta+\xi f_{1}(\theta)-\xi u_{1}^{\prime} .
$$


The mean Lagrangian functions of the gases into which it dissociates are respectively

and

$$
\eta R_{2} \theta \log \frac{z \rho_{0}^{\prime}}{\eta}+\eta f_{2}(\theta)-\eta u_{2}^{\prime},
$$

$$
\zeta R_{3} \theta \log \frac{v \rho_{0}^{\prime \prime}}{\zeta}+\zeta f_{3}(\theta)-\zeta w_{3} .
$$

Then from the condition

$$
d H=0,
$$

we get, since

$$
\begin{gathered}
d \xi=-(d \eta+d \zeta), \\
\frac{d v}{d \xi}=-\frac{\mathbf{I}}{\sigma}, \\
\frac{d \eta}{c_{2}}=\frac{d \zeta}{c_{3}},
\end{gathered}
$$

and

where $c_{2}$ and $c_{3}$ are the combining weights of the gases into which the solid dissociates, and $\sigma$ the density of the solid or liquid

$$
{ }_{v^{2}}^{\eta}=\phi_{1}(\theta) \epsilon^{\frac{\left(\left(c_{2}+c_{3}\right) w_{1}-c_{2} w_{2}-c_{8} w_{3}\right)}{c_{2} R_{2} \theta}+\left(c_{2}+c_{3}\right)\left(\frac{\eta}{c_{3}}+\frac{\zeta}{c_{3}}\right) \frac{1}{\sigma v}} .
$$

It follows from this equation that, as before, dissociation is hindered by the presence in excess of either of the results of the dissociation.

In this case the dissociation would be affected to a small extent by the presence of a neutral gas, for if the system is confined in a closed vessel the volume of the solid or liquid diminishes as it evaporates, the neutral gas above it expands, and its Lagrangian function therefore increases. Hence we see by $\S(84)$ that the presence of the neutral gas will increase the dissociation. 
By an investigation similar to that in $\S(89)$ we can easily show that if $\kappa$ denotes the value of $\eta \zeta / \gamma^{2}$, and $\delta \kappa$ the change produced by the presence of the neutral gas, then

$$
\frac{\delta \kappa}{\kappa}=\frac{c_{3}+c_{2}}{c} \frac{\epsilon}{v \sigma},
$$

where $\epsilon$ is the mass of the neutral gas, $c$ its combining weight and $\sigma$ the density of the solid or liquid. Since $\epsilon / v \sigma$ is the ratio of the mass of the gas to the mass of the same volume of the dissociable solid, we see that the effect produced by the neutral gas, unless its pressure amounts to some hundreds of atmospheres, is extremely small. If we take the case of sal-ammoniac, where $\sigma$ is about $\mathrm{I}^{\prime} 5$, we see that for a pressure of 100 atmospheres

$$
\frac{\delta \kappa}{\kappa}=3 \text { approximately, }
$$

so that if the pressure were increased by about 3.3 atmospheres the change in $\kappa$ would be about one per cent.

I Io. Dissociation of Salts in Solution. We have seen $\S 92$ that Van 't Hoff has given reasons for believing that the molecules of a salt in a dilute solution exert the same pressure as they would if they were in the gaseous state at the same temperature and volume: and that the mean Lagrangian function of the molecules in the solution is therefore the same as that of the same number of gaseous molecules. We might therefore expect from analogy that in some cases these molecules would be dissociated though the effects of this dissociation might not be so recognisable as in the case of gases. Many cases of the dissociation of salts in solution have been observed, sodium sulphate and the ammonium salts are well-known examples (Muir's Principles of Chemistry, p. 367). Indeed the theory has 
recently been started that in dilute aqueous solutions the dissolved acid or salt is in most cases dissociated and that to a very considerable extent ; thus it has been stated that in dilute solutions of $\mathrm{HCl}$ as much as $9 \circ$ per cent. of the acid is dissociated. The reasons given for this conclusion do not seem to me to be very convincing, and the experimental results on which they are based seem to admit of a different interpretation. The supporters of this theory urge that for the salt to produce the effect which in some cases it does, it is necessary to suppose that the molecules of the salt exert a greater pressure than they would if they occupied the same volume at the same temperature when in the gaseous condition. This reasoning is founded on the assumption that all the effects due to the dissolved salt may be completely explained merely by supposing the volume occupied by the solvent to be filled with the molecules of the salt in the gaseous condition. Now though we may admit that the salt does produce the effects that would be produced by this hypothetical distribution of gaseous molecules, still it does not follow that these are the only effects produced by the salt. The salt may change the properties of the solvent and the effects attributed to the dissociation of the molecules may in reality be due to this change. The investigation in $\S 97$ proves that this must be so in some cases, for we saw that the effects of the addition of salt on the compressibility of the solution were much too large to be explained by any amount of dissociation.

In the case of the dissociation of salt solutions the properties of the solution might alter as the dissociation progressed. Thus the dissociation might alter the surface tension of the solution, in which case the amount of dissociation would depend upon the shape and volume of the solution; or it might alter the coefficient of compressibility or the volume 
of the solution, and then the amount of dissociation would be influenced by external pressure. In fact the dissociation of the dissolved salt would probably be much more susceptible to external physical influences than the dissociation of a gas. We shall however discuss these as particular cases of the next investigation, which deals with a much more general case of chemical equilibrium between either gases or dilute solutions. 


\section{CHAPTER XIV.}

GENERAL CASE OF CHEMICAL EQUILIBRIUM.

I I I. THE case we shall consider in this chapter is the equilibrium of four substances $A, B, C, D$, either gases or in dilute solutions, such that $A$ by its action on $B$ produces $C$ and $D$, while $C$ by its action on $D$ produces $A$ and $B$.

A well-known example of this kind of action is the case in which the four substances $A, B, C, D$ are respectively nitric acid, sodium sulphate, sulphuric acid and sodium nitrate: the nitric acid acts on the sodium sulphate and forms sodium nitrate and sulphuric acid, while the sulphuric acid acts on the sodium nitrate and forms sodium sulphate and nitric acid.

The problem we have to discuss is to find, when any quantities of four such substances are mixed together, the quantity of each when there is equilibrium.

Let $\xi, \eta, \zeta, \epsilon$ be the masses of the substances $A, B, C$, $D$ respectively, $w_{1}, w_{2}, w_{3}, w_{4}^{\prime}$ the mean potential energy of unit mass of each of these substances, $w$ the mean potential energy of the mixture. Let us suppose that each of these substances obeys Boyle's law; and $\rho$ denoting the density 
of any one at the temperature $\theta$ and pressure $p$, let the fundamental equation of $A$ be

that of $B$

$$
p=R_{1} \rho \theta
$$

that of $C$

$$
p=R_{\mathrm{a}} \rho \theta
$$

and that of $D$

$$
\begin{aligned}
& p=R_{3} \rho \theta, \\
& p=R_{4} \rho \theta .
\end{aligned}
$$

Then the mean Lagrangian functions of $A, B, C$ and $D$ are respectively

$$
\begin{aligned}
& \xi R_{1} \theta \log \frac{v \rho_{0}}{\xi}+\xi f_{1}(\theta)-\xi w_{1}, \\
& \eta R_{9} \theta \log \frac{v \rho_{0}^{\prime}}{\eta}+\eta f_{2}(\theta)-\eta w_{2}, \\
& \zeta R_{3} \theta \log \frac{v \rho_{0}^{\prime \prime}}{\zeta}+\xi f_{3}(\theta)-\zeta w_{3}, \\
& \epsilon R_{4} \theta \log \frac{v \rho_{0}^{\prime \prime \prime}}{\epsilon}+\epsilon f_{4}(\theta)-\epsilon w_{4}^{\prime},
\end{aligned}
$$

where $v$ is the volume in which the substances are confined.

The above expressions represent the mean Lagrangian functions equally well whether the substances $A, B, C, D$ are gases or dilute solutions, provided the solutions are so dilute that the molecules of the substances dissolved in them exercise the same pressure as they would if placed at the same temperature in the same volume when empty.

If we are considering solutions we shall require the mean Lagrangian function of the solvent, for the properties of this may alter as chemical combination goes on. If $\pi$ is the mass of the solvent, $w_{5}$ the potential energy of unit mass, then its mean I,agrangian function will be of the form

$$
\pi \gamma \theta+\pi f_{5}(\theta)-\pi \boldsymbol{z}^{\prime}{ }^{\circ}
$$

We must now investigate the relations between the changes in $\xi, \eta, \zeta, \epsilon$ as chemical action goes on. 
Let us denote by $(A)$ the molecule of the substance $A$, with a similar notation for the other molecules, and let the chemical action which goes on between the four substances be represented by

$$
a(A)+b(B)=c(C)+d(D) \ldots \ldots \ldots \ldots(2 \mathrm{I} 3) .
$$

Thus, in the case of the mixture of sulphuric and nitric acids, sodium nitrate and sodium sulphate, since the equation which expresses the reaction is

$$
2 \mathrm{HNO}_{3}+\mathrm{Na}_{8} \mathrm{SO}_{4}=\mathrm{H}_{8} \mathrm{SO}_{4}+2 \mathrm{NaNO}_{3},
$$

if the molecules of nitric acid, sodium sulphate, sulphuric acid and sodium nitrate in the solution are represented respectively by $\mathrm{HNO}_{3}, \mathrm{Na}_{2} \mathrm{SO}_{4}, \mathrm{H}_{2} \mathrm{SO}_{4}$ and $\mathrm{NaNO}_{3}$, then $a=2, b=\mathrm{I}, c=\mathrm{I}, d=2$. If however the molecules of these substances are represented by $\mathrm{H}_{2} \mathrm{~N}_{2} \mathrm{O}_{6}, \mathrm{Na}_{2} \mathrm{SO}_{4}, \mathrm{H}_{2} \mathrm{SO}_{4}$, $\mathrm{Na}_{8} \mathrm{~N}_{2} \mathrm{O}_{6}$, then $a=b=c=d=\mathrm{I}$.

Thus we see that it is necessary to know the structure of the molecule as well as its relative composition.

From equation (2 14) we see that if $a$ molecules of $A$ disappear it must be because they have combined with $b$ of $B$ to produce $c$ of $C$ and $d$ of $D$, so that $b$ molecules of $B$ have also disappeared, while $c$ of $C$ and $d$ of $D$ have appeared.

Let $\delta_{1}, \delta_{2}, \delta_{3}, \delta_{4}$ represent the relative densities of $A, B, C, D$ at the same temperatures and pressures, then

$$
R_{1} \delta_{1}=R_{2} \delta_{2}=R_{2} \delta_{3}=R_{4} \delta_{4} \ldots \ldots \ldots \text {....(214). }
$$

If the masses of $A, B, C, D$ are altered by $d \xi, d \eta, d \zeta, d \epsilon$ respectively, then the alterations in the number of molecules of $A, B, C, D$ are respectively proportional to

$$
\frac{d \xi}{\delta_{1}}, \frac{d \eta}{\delta_{z}}, \frac{d \zeta}{\delta_{3}}, \frac{d \epsilon}{\delta_{4}}
$$


Thus, since the alterations in the number of molecules are proportional to $a, b,-c,-d$ respectively, we have

$$
\frac{d \xi}{a \delta_{1}}=\frac{d \eta}{b \delta_{2}}=-\frac{d \zeta}{c \delta_{3}}=-\frac{d \epsilon}{d \delta_{4}} .
$$

So that

$$
\left.\begin{array}{l}
\frac{d \eta}{d \xi}=\frac{b \delta_{2}}{a \delta_{1}} \\
\frac{d \zeta}{d \xi}=-\frac{c \delta_{3}}{a \delta_{1}} \\
\frac{d \epsilon}{d \xi}=-\frac{d \delta_{4}}{a \delta_{1}}
\end{array}\right\}
$$

Now when the system is in equilibrium the value of the Hamiltonian function must be stationary, so that if we suppose the equilibrium displaced by the quantity $d \xi$ of $A$ combining with the proper quantity of $B$ the change in the Hamiltonian function must be zero, hence we must have

$$
d H=0
$$

Let us take first the case when $A, B, C, D$ are gases, then since $H$ is the sum of the mean Lagrangian function for these substances the condition (216) with the help of equations (2I5) gives the equation

$$
\begin{gathered}
a \delta_{1} R_{1} \theta \log \frac{v \rho_{0}}{\xi}-a \delta_{1} R_{1} \theta+b \delta_{2} R_{2} \theta \log \frac{v \rho_{0}^{\prime}}{\eta}-b \delta_{2} R_{2} \theta \\
-c \delta_{3} R_{3} \theta \log \frac{v \rho_{0}^{\prime \prime}}{\zeta}+c \delta_{3} R_{3} \theta-d \delta_{4} R_{4} \theta \log \frac{v \rho_{0}^{\prime \prime \prime}}{\epsilon}+d \delta_{4} R_{4} \theta \\
+a \delta_{1} f_{1}(\theta)+b \delta_{2} f_{2}(\theta)-c \delta_{3} f_{3}(\theta)-d \delta_{4} f_{4}(\theta)-a \delta_{1} \frac{\dot{d} w}{d \xi}=0, \\
w=\xi w_{1}+\eta v u_{2}+\zeta w_{3}+\epsilon w_{4} .
\end{gathered}
$$

where

Then by (214) we may write this equation in the form 


$$
\frac{\zeta^{c} \epsilon^{d}}{\xi^{a} \eta^{b}}=v^{c+a-a-b} \phi(\theta) \epsilon \frac{a}{\frac{d}{R_{1} \theta}} \frac{d w}{d \xi}
$$

when $\phi(\theta)$ is a function of $\theta$ but does not involve $\xi, \eta, \zeta$ or $\epsilon$.

I I . In the case of dilute solutions the equation corresponding io $(2 \mathrm{I} 7)$ is easily seen to be

$$
\frac{\zeta^{c} \epsilon^{d}}{\xi^{a} \eta^{b}}=\phi(\theta) \tau^{c+d-a-b} \epsilon^{\frac{a}{R_{1} \theta}} \frac{d w}{d \xi} \epsilon^{-\frac{a}{R_{1} \theta}} \frac{d Q}{d \xi} \ldots . .
$$

where $Q$ is the mean Lagrangian function of the solvent and equals

$$
\pi \gamma \theta+\pi f_{5}(\theta)-\pi \omega_{5}
$$

The value of $d Q / d \xi$ will be zero if the properties of the solvent do not change as chemical action goes on; in any case since the solutions are very dilute the properties of the solvent may be assumed to be changed by an amount proportional to the quantity of salt dissolved, $Q$ will therefore be a linear function of $\xi, \eta, \zeta, \epsilon$ and $d Q / d \xi$ will not involve any of these quantities, and in this case as in the former one we have

$$
\frac{\xi^{c} \epsilon^{d}}{\xi^{a} \eta^{b}}=\phi_{1}(\theta) v^{c+d-a-b} \epsilon^{\frac{a}{R_{1} \theta}} \frac{d w}{d \xi}
$$

so that the equations of equilibrium for gases and dilute solutions are of exactly the same form.

I I3. The value of $d w / d \xi$ measures the increase in the potential energy of the system when the mass of $\xi$ is increased by unity. Now if heat is produced when $C$ and $D$ combine to form $A$ and $B$, the potential energy diminishes as $\xi$ increases, and when the quantity of heat is large its mechanical equivalent may be taken as a measure of the decrease in the potential energy.

If the combination of $C$ and $D$ is accompanied by the production of heat, $d w / d \xi$ is negative, and we see therefore 
that if $\theta=0$

$$
\zeta^{c} \epsilon^{d} / \xi^{a} \eta^{b}=0,
$$

or either $\zeta$ or $\epsilon$ must vanish, that is, the combination of $C$ and $D$ will go on until one of these substances gives out, in other words the reaction attended by the production of heat will at the zero of absolute temperature go on as far as possible.

According to Berthelot's law of "Maximum Work" the reaction accompanied by the formation of heat goes on as far as possible at all temperatures, the equation ( 2 r 8 ) however shows that this is strictly true only at the zero of temperature.

For substances which give out large quantities of heat when they combine equation (218) shows that the combination increases so rapidly as the temperature diminishes, that if there is any combination at all at temperatures as high as $1000^{\circ} \mathrm{C}$., Berthelot's law will be practically true at all ordinary temperatures. To illustrate this let us take the case of hydrogen and oxygen, where the combination is represented by the equation

$$
2 \mathrm{H}_{2}+\mathrm{O}_{2}=2 \mathrm{H}_{2} \mathrm{O} \text {. }
$$

Let $\xi, \eta, \zeta$ be the quantities of hydrogen, oxygen and water respectively, then $a=2, b=\mathrm{I}, c=2, d=0$, and equation (2 I 8) becomes

$$
\frac{\zeta^{2}}{\xi^{2} \eta}=\frac{\mathrm{I}}{v} \phi(\theta) \epsilon^{\frac{2}{R_{1} \theta}} \frac{d w}{d \xi} .
$$

If we substitute for $f(\theta)$ its value given on page 270 we shall find that $\phi(\theta)$ in this case $=C / \theta^{3 \cdot 5}$. For hydrogen at $0^{\circ} \mathrm{C} . R_{1} \theta=\mathbf{I} \cdot \mathbf{I} \times 10^{10}$, and since in the combination of one gramme of hydrogen with oxygen 34000 calories are given out,

$$
\frac{d w}{d \xi}=1 \cdot 43 \times 10^{12} \text {. }
$$

Let us suppose that equivalent quantities of hydrogen and oxygen are mixed together, and that the number of equivalents which combine to form water is to the whole 
number of equivalents of either oxygen or hydrogen present initially as $x$ to $\mathrm{I}$, then $x$ is given by the equation

$$
\frac{x^{2}}{(\mathrm{I}-x)^{3}}=\frac{C^{\prime}}{\theta^{3.5}} \underset{R_{1}^{2} \theta}{2} \frac{d z}{d \xi} \text {. }
$$

Suppose that at $\operatorname{rog2}^{\circ} \mathrm{C}$. one half of the equivalents combine, then the value of $x$ at $546^{\circ} \mathrm{C}$. is given by the equation

$$
\frac{x^{2}}{(1-x)^{3}}=2 \cdot\left(\frac{5}{3}\right)^{3 \cdot 5} \epsilon^{2\left(\frac{130}{3}-\frac{130}{5}\right)},
$$

thus approximately

$$
\begin{aligned}
\mathrm{I}-x & =\frac{\mathrm{I}}{5} \epsilon^{-\frac{34}{3}} \\
& =\frac{\mathrm{I}}{5} \times 10^{-5} .
\end{aligned}
$$

So that at this temperature only about one in five hundred thousand of the molecules will be left uncombined. Thus in a case like this very considerable dissociation at one temperature is compatible with almost complete combination at a temperature not very much lower.

I 4. The effect of pressure on chemical equilibrium. We have by equation (219)

thus if

$$
\frac{\xi^{c} \epsilon^{d}}{\xi^{a} \eta^{b}}=v^{c+d-a-b} \phi(\theta) \underset{\epsilon}{\stackrel{a}{R_{1} \theta} \frac{d z w}{d \xi}}
$$

the ratio $\zeta^{c} \epsilon^{d} / \xi^{a} \eta^{b}$ is independent of the volume, so that if we mix given quantities of the four substances the amount of chemical action which will go on will be independent of the volume into which the substances are put. Since the chemical reaction is such that when $A$ acts on $B, a$ molecules of $A$ and $b$ of $B$ disappear while $c$ of $C$ and $d$ of $D$ are produced, we see that if $a+b=c+d$ the number of molecules in the vessel does not change as the reaction goes on. This is sometimes expressed by saying that the combination 
takes place without change of volume, and in this case, as we have just seen, the amount of chemical combination is not affected by the volume in which the combining substances are placed. If $a+b$ is greater than $c+d$ then the larger the volume $v$, the smaller will be the ratio of $\zeta^{c} \epsilon^{d}$ to $\xi^{a} \eta^{b}$. Now the action of $C$ on $D$ tends to diminish this ratio, while that of $A$ on $B$ tends to increase it, and if $a+b$ is greater than $c+d$ the number of molecules is increased when $C$ acts on $D$ and diminished when $A$ acts on $B$. Thus we see from equation (2 I9) that when chemical combination alters the number of molecules the state of equilibrium depends upon the volume within which the substances are confined, and that the effect of increasing the volume is to favour that reaction which is accompanied by an increase in the number of molecules. In other words, the chemical action which produces an increase in volume is hindered by pressure, while that which produces a diminution is helped by it. This is another example of the law stated in $\$(84)$.

I 5. Let us now consider a little more closely some of the results of equation ( 2 I 9 ), taking for the sake of simplicity the case when $a=b=c=d=\mathbf{I}$.

Let us suppose that the masses of the four substances $A, B, C, D$ before combination begins are $\xi_{0}, \eta_{0}, \zeta_{0}, \epsilon_{0}$, and that when they have reached the state of equilibrium a quantity $\delta_{1} p$ of $A$ has disappeared, then by equations (2 I 5) we have

$$
\begin{aligned}
& \xi=\xi_{0}-\delta_{1} p \\
& \eta=\eta_{0}-\delta_{2} p \\
& \zeta=\zeta_{0}+\delta_{3} p \\
& \epsilon=\epsilon_{0}+\delta_{4} p ;
\end{aligned}
$$

and equation (2 I9) becomes

$$
\frac{\left(\xi_{0}+\delta_{3} p\right)\left(\epsilon_{0}+\delta_{4} p\right)}{\left(\xi_{0}-\delta_{1} p\right)\left(\eta_{0}-\delta_{2} p\right)}=\phi \phi(\theta) \epsilon \frac{a}{R_{1} \theta} \frac{d w}{d \xi} \ldots \ldots(220)
$$


Let us suppose that the quantities of the substances mixed together initially were proportional to their combining weights, i.e. that initially equivalent quantities of the four substances were taken, then we may put

$$
\xi_{0}=\delta_{1} t, \eta_{0}=\delta_{2} t, \zeta_{0}=\delta_{3} t, \epsilon=\delta_{4} t
$$

And equation (220) becomes

$$
\frac{\delta_{3} \delta_{4}(t+p)^{2}}{\delta_{1} \delta_{2}(t-p)^{2}}=\phi(\theta)_{\epsilon} \stackrel{\frac{a}{R_{1} \theta}}{\frac{d w}{d \xi}} ;
$$

if we put

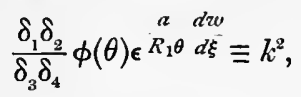

then

$$
k=\frac{t+p}{t-p}
$$

and is called the affinity coefficient of the reaction (Muir's Principles of Chemistry, p. 4I 7). Thus we may write equation (220) in the form

$$
\frac{\left(\zeta_{0}+\delta_{3} p\right)\left(\epsilon_{0}+\delta_{4} p\right)}{\left(\xi_{0}-\delta_{1} p\right)\left(\eta_{0}-\delta_{2} p\right)}=k^{2} \frac{\delta_{3} \delta_{4}}{\delta_{1} \delta_{2}}
$$

where $k$ is constant as long as the temperature remains unchanged.

The effects due to what are called "mass actions," that is the effects produced by varying the quantities of the four substances initially present may be deduced at once from this equation.

Let $\delta p_{1}$ be the increase in $p$ when $\xi_{0}$ is increased by $\delta \xi_{0}$, the quantities $\eta_{0}, \zeta_{0}, \epsilon_{0}$ remaining constant; and let $\delta p_{2}, \delta p_{3}$, $\delta p_{4}$ be the respective increases when $\eta_{0}, \zeta_{0}, \epsilon_{0}$ are increased by $\delta \eta_{0}, \delta \xi_{0}, \delta \epsilon_{0}$ respectively. Then we get at once from equation $(2 \mathrm{I})$

$$
\gamma \delta p_{1}=\frac{\delta \xi_{0}}{\xi_{0}-\delta_{1} p}=\frac{\delta \xi_{0}}{\dot{\xi}}
$$




$$
\begin{aligned}
& \gamma \delta p_{2}=\begin{array}{l}
\delta \eta_{0} \\
\eta_{0}-\delta_{2} p
\end{array}=\frac{\delta \eta_{0}}{\eta}, \\
& \gamma \delta p_{3}=-\frac{\delta \zeta_{0}}{\zeta_{10}+\delta_{3} p}=-\frac{\delta \zeta_{0}}{\zeta}, \\
& \gamma \delta p_{4}=-\frac{\delta \epsilon_{0}}{\epsilon_{0}+\delta_{4} p}=-\frac{\delta \epsilon_{0}}{\epsilon} ;
\end{aligned}
$$

where $\gamma$ is the positive quantity

or

$$
\begin{gathered}
\frac{\delta_{1}}{\xi_{0}-\delta_{1} p}+\frac{\delta_{2}}{\eta_{0}-\delta_{2} p}+\frac{\delta_{3}}{\zeta_{0}+\delta_{3} p}+\frac{\delta_{4}}{\epsilon_{0}+\delta_{1} p}, \\
\frac{\delta_{1}}{\xi}+\frac{\delta_{2}}{\eta}+\frac{\delta_{3}}{\zeta}+\frac{\delta_{4}}{\epsilon} .
\end{gathered}
$$

We see from these equations that $\delta p_{1} / \delta \xi_{0}$ and $\delta p_{2} / \delta \eta_{0}$ are positive while $\delta p_{3} / \delta \zeta_{0}, \delta p_{4} / \delta \epsilon_{0}$ are negative, so that any increase - in the quantity of $A$ and $B$ initially present increases the amount of combination that goes on between these substances, while any increase in the quantities of $C$ and $D$ initially present decreases the amount of combination, and further that the effects of equal small changes in the masses of $A, B, C, D$ before combination takes place are inversely proportional to the amount of these substances present in the state of equilibrium.

In the more general case, where $a, b, c, d$ are not each put equal to unity, we may easily prove that

$$
\begin{aligned}
& \gamma \delta p_{1}=\frac{a \delta \xi_{0}}{\xi}, \\
& \gamma \delta p_{2}=\frac{b \delta \eta_{0}}{\eta}, \\
& \gamma \delta p_{3}=-\frac{c \delta \xi_{0}}{\zeta}, \\
& \gamma \delta p_{4}=-\frac{d \delta \epsilon_{0}}{\epsilon} ;
\end{aligned}
$$


and that if $\delta p$ be the change in $p$ due to an increase $\delta r$, in volume, everything else being constant,

$$
\begin{aligned}
& \gamma \delta p=(c+d-a-b) \frac{\delta v}{v}, \\
& \gamma=\frac{a \delta_{1}}{\xi}+\frac{b \delta_{2}}{\eta}+\frac{c \delta_{3}}{\zeta}+\frac{d \delta_{4}}{\epsilon}
\end{aligned}
$$

where

here $a \delta_{1} p$ is the mass of $A$ which has disappeared.

I 6. The expression (22I) agrees with the formula obtained by Guldberg and Waage from quite different principles (see Muir's Principles of Chemistry, p. 407, and Lothar Meyer, Modernen Theorien der Chemie, chap. XIII). The case when $a=b=c=d$ is the only one however in which the expression deduced from Hamilton's principle agrees with that given by Guldberg and Waage. According to their theory, as given in the works we have just cited, the equation (221) is always true, while according to the theory we have been explaining it is only true when $a=b=c=d$. It would seem however that the principles from which Guldberg and Waage deduced their equations would when $a, b, c$ and $d$ are not all equal lead to equation (2I9) rather than (22I), for their point of view seems to be as follows. Consider first the case when $a=b=c=d=\mathbf{1}$, then in a certain proportion of the collisions which occur between the molecules of $A$ and $B$, chemical combination between $A$ and $B$ will take place. The number of collisions in unit time is proportional to the product of the numbers of molecules of $A$ and $B$, and so is proportional to $\xi \eta$. The number of cases in which combination takes place may be taken therefore to be $k \xi \eta$ when $k$ is a quantity which is independent of the quantities of $A, B, C, D$ present. In other words, the number of molecules which leave the $A, B$ states and enter those of $C$ and $D$ is $k \xi_{\eta}$; in a similar way we can see that the number of molecules of $C$ and $D$ which become $A$ and

T. D. 
$B$ is $k^{\prime} \zeta_{\epsilon}$. Now when the system is in a steady state the number of molecules of $A$ and $B$ formed must be the same as the number which disappear, and therefore

$$
k \xi \eta=k^{\prime} \zeta \epsilon
$$

which is Guldberg's and Waage's equation. We can easily see however that the above reasoning is only applicable when chemical combination takes place between one molecule of $A$ and one of $B$, and again between one of $C$ and one of $D$, or in other words when $a=b=c=d=\mathrm{r}$. If on the other hand the equation which represents the chemical reaction is

$$
2(A)+(B)=(C)+2(D),
$$

then chemical combination will take place when one molecule of $B$ is in collision with two of $A$ simultaneously; the number of such combinations will be proportional to $\eta \xi^{2}$ and not to $\eta \xi$, and thus the number of molecules of $A$ which disappear owing to their combination with $B$ molecules may be represented by $k \eta \xi^{2}$; similarly the number of molecules of $D$ which disappear and of $A$ which appear by the combination of $C$ and $D$ may be represented by $k^{\prime} \zeta \epsilon^{2}$; and since in the state of equilibrium the number of molecules of $A$ which disappear must be the same as the number which appear we must have

$$
k \eta \xi^{2}=k^{\prime} \zeta^{2}
$$

which agrees with equation (2x9) but not with Guldberg and Waage's equation.

II7. As we noticed before in $\S(1 \circ 7)$, there is some ambiguity as to what the molecule of the dissolved salt or acid really is. For example, take the case already mentioned where the reaction is represented by the equation

$$
\mathrm{H}_{2} \mathrm{SO}_{4}+2 \mathrm{NaNO}_{3}=2 \mathrm{HNO}_{3}+\mathrm{Na}_{2} \mathrm{SO}_{4} \text {, }
$$

we do not know whether the molecule of sodium nitrate is 
represented by $\mathrm{NaNO}_{3}$ or by $\mathrm{Na}_{2} \mathrm{~N}_{2} \mathrm{O}_{6}$, or whether the molecule of nitric acid is represented by $\mathrm{HNO}_{3}$ or $\mathrm{H}_{2} \mathrm{~N}_{2} \mathrm{O}_{6}$. This point could probably be settled by experiments on osmotic pressure, the lowering of the vapour pressure of the solution and the effect of the salt or acid upon the freezing point. If the molecules are represented by $\mathrm{Na}_{2} \mathrm{~N}_{2} \mathrm{O}_{6}, \mathrm{H}_{2} \mathrm{~N}_{8} \mathrm{O}_{6}$ and not by $\mathrm{NaNO}_{3}, \mathrm{HNO}_{3}$, it would be necessary to dissolve I 70 and I 26 grammes of these substances in a litre of water, instead of 85 and 63 to produce the effects observed in solutions of one gramme equivalent per litre.

We can however use the formula (219) giving the amount of chemical action between these substances to decide this point. If the molecules are represented by $\mathrm{HNO}_{3}, \mathrm{Na}_{2} \mathrm{SO}_{4}$, $\mathrm{H}_{2} \mathrm{SO}_{4}$ and $\mathrm{NaNo}_{3}$ then by equation (2 I9) $\epsilon \zeta^{2} / \xi \eta^{2}$ is constant provided the temperature remains unaltered, if however the molecules are represented by $\mathrm{H}_{2} \mathrm{~N}_{2} \mathrm{O}_{6}, \mathrm{Na}_{2} \mathrm{SO}_{4}, \mathrm{H}_{2} \mathrm{SO}_{4}$, and $\mathrm{Na}_{2} \mathrm{~N}_{2} \mathrm{O}_{6}$ (or by $\mathrm{HNO}_{3}, \frac{1}{2} \mathrm{Na}_{2} \mathrm{SO}_{4}, \frac{1}{2} \mathrm{H}_{2} \mathrm{SO}_{4}, \mathrm{NaNO}_{3}$ ) then $\epsilon \zeta / \xi \eta$ is constant as long as the temperature is unaltered, where $\xi, \eta, \zeta, \epsilon$ are the masses of the sulphuric acid, sodium nitrate, nitric acid and sodium sulphate respectively.

This reaction has been investigated by Thomsen (Thermochemische Untersuchungen I. p. I 2 I) and in the following table

\begin{tabular}{l|l|l}
\hline$n$ & $\epsilon \zeta / \xi \eta$ & $\epsilon \zeta^{2} / \xi \eta^{2}$ \\
\hline 8 & 2.07 & 40.5 \\
4 & 2.6 & 33 \\
2 & 2.5 & $\mathbf{I} 3.05$ \\
$\mathbf{I}$ & 3.3 & 8 \\
$\frac{1}{2}$ & $4 . \mathrm{I}$ & 3.2 \\
$\frac{1}{4}$ & $4 . \mathrm{I}$ & $\mathbf{I} .0$ \\
\hline
\end{tabular}

the values of $\epsilon \zeta^{2} / \xi \eta^{2}, \epsilon \zeta / \xi \eta$, calculated from his experiments 
for different proportions of the substances, are given. $n$ is the ratio of the number of equivalents of sodium sulphate to the number of equivalents of nitric acid before chemical combination commences.

It will be seen from this table that when there is only a very small quantity of nitric acid present initially, formula (219) seems to agree with the observations as well as (22I), but that it ceases to be any approximation when the solution gets stronger, and that now equation (22I) agrees better with the experiments. From this we should conclude that in very dilute solutions the molecules of nitric acid and sodium nitrate may possibly be represented by $\mathrm{HNO}_{3}$, $\mathrm{NaNO}_{3}$, but that in stronger solutions either they are represented by $\mathrm{H}_{2} \mathrm{~N}_{2} \mathrm{O}_{6}, \mathrm{Na}_{2} \mathrm{~N}_{2} \mathrm{O}_{6}$, or else that the molecules of sulphuric acid and sodium sulphate are represented by $\frac{1}{2} \mathrm{H}_{2} \mathrm{SO}_{4}, \frac{1}{2} \mathrm{Na}_{2} \mathrm{SO}_{4}$. Pfeffer's determination $\S\left(9^{8}\right)$ of the osmotic pressure produced by a potassium sulphate solution suggests that the molecule is represented by $\frac{1}{2}\left(\mathrm{~K}_{2} \mathrm{SO}_{4}\right)$. We ought not however to attach as much weight to the experiments with dilute solutions as to those with strong, because in the weak solutions a very small error in the determinations will produce a considerable error in the value of $\epsilon \zeta^{2} / \xi \eta^{2}$ or $\epsilon \zeta / \xi \eta$.

If there was any change of this kind in the constitution of the molecules as the strength of the solution increased it would probably show itself in the effect of the substance on the osmotic pressure, on the vapour pressure, and on the lowering of the freezing point, even though these effects were complicated by the alteration in the properties of the solvent produced by the addition of the salt.

I 8 . In the case we have just been considering the four substances $A, B, C, D$ were supposed to be either 
gaseous or soluble. We must now see how the equations have to be modified when one or more of the substances is a solid, and if we are considering the case of solutions an insoluble one.

Let us take first the case when only one of the substances $D$ is an insoluble solid, for example when the four bodies are oxalic acid, calcium chloride, hydrochloric acid, and calcium oxalate.

The mean Lagrangian function for $D$ will now be of the form

and the condition

$$
\epsilon \gamma \theta+\epsilon f(\theta)-\epsilon \tau \mathcal{U}_{4}
$$

$$
\frac{d H}{d \xi}=0
$$

will lead to the equation

$$
\frac{\zeta^{c}}{\xi^{a} \eta^{b}}=v^{c-a-b} \phi(\theta) \epsilon^{\frac{a}{R_{1} \theta}} \frac{d z v}{d \xi}
$$

If two of the substances are insoluble solids, as for example when $A$ is potassium carbonate, $B$ barium sulphate, $C$ potassium sulphate, $D$ barium carbonate, then we can easily prove that

$$
\frac{\xi^{c}}{\xi^{a}}=\eta^{c-a} \phi(\theta) \epsilon^{\frac{a}{R_{1} \theta} \frac{d w}{d \xi}}
$$

We see from these equations that the amount of combination which goes on does not depend on the masses of the insoluble substances.

I 19. As an example of a case where the conditions are rather more complicated than in those discussed in the last paragraph, we shall consider a case investigated by Horstmann (Watts' Dictionary of Chemistry, 3rd Supplement, p. 433) where hydrogen, carbonic oxide and water were exploded, and water and carbonic acid produced. 
Here we have to consider five substances, hydrogen, carbonic oxide, oxygen, water, and carbonic acid; let $\xi, \eta$, $\zeta, \epsilon, \pi$, be the masses of these substances respectively, and let $c_{1}, c_{2}, \ldots c_{5}$, be their molecular weights.

Let the relation between the pressure $p$, the density $\rho$, and the absolute temperature $\theta$ be for hydrogen

for carbonic acid

$$
p=R_{1} \theta \rho,
$$

$$
p=R_{\mathbf{2}} \theta \rho,
$$

with a corresponding notation for the others.

Let the mean Lagrangian function for the hydrogen be

$$
\xi R_{1} \theta \log \frac{v \rho_{0}}{\xi}+\xi f_{1}(\theta)-\xi u_{1}
$$

where $v$ is the volume in which the gases are confined, and $w_{1}$ the mean potential energy of unit mass of hydrogen. The mean Lagrangian function of the other gases will be given by analogous expressions.

Now whatever changes go on among the various gases we have since the quantity of hydrogen is constant

$$
\frac{\xi}{c_{1}}+\frac{\epsilon}{c_{4}}=\text { a constant }
$$

since the carbon is constant

$$
\frac{\eta}{c_{2}}+\frac{\pi}{c_{b}}=\text { a constant }
$$

since the oxygen is constant

$$
\frac{1}{2} \frac{\eta}{c_{2}}+\frac{\zeta}{c_{3}}+\frac{1}{2} \frac{\epsilon}{c_{4}}+\frac{\pi}{c_{5}}=\text { a constant }
$$

these are three equations between five unknown quantities, so that if we give arbitrary variations to two of them the variations of the others will be determinate. 
Let us choose $\xi$ and $\eta$ as the independent variables, then when $\eta$ is constant we have

$$
\frac{d \zeta}{d \xi}=\frac{1}{2} \frac{c_{3}}{c_{1}}, \frac{d \epsilon}{d \xi}=-\frac{c_{4}}{c_{1}}, \frac{d \pi}{d \xi}=0,
$$

and wheil $\xi$ is constant

$$
\frac{d \zeta}{d \eta}=\frac{1}{2} \frac{c_{3}}{c_{2}}, \frac{d \epsilon}{d \xi}=0, \frac{d \pi}{d \xi}=-\frac{c_{5}}{c_{2}} .
$$

When the system is in equilibrium the mean Lagrangian function is stationary for all possible variations, so that we must have

$$
\left(\frac{d H}{d \xi}\right)_{\eta \text { constant }}=0, \quad\left(\frac{d H}{d \eta}\right)_{\xi \text { constant }}=0 .
$$

Remembering that

$$
R_{1} c_{1}=R_{2} c_{9}=R_{3} c_{3}=R_{4} c_{4}=R_{5} c_{5},
$$

the first equation gives

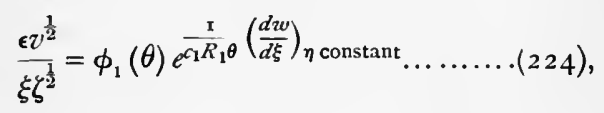

and the second

$$
\frac{\pi v^{\frac{1}{2}}}{\eta \zeta^{\frac{1}{2}}}=\phi_{2}(\theta) e^{\frac{x}{c_{1} R_{1} \theta}\left(\frac{d z w}{d \eta}\right)_{\xi \text { constant }}}
$$

where $w$ is the mean potential energy of the mixture of gases.

These are of the same form as the equations I obtained from kinematical considerations alone in my paper on the Chemical Combination of Gases already referred to.

If we divide $(224)$ by $(225)$ we get

$$
\frac{\epsilon \eta}{\xi \pi}=\frac{\phi_{1}(\theta)}{\phi_{\mathbf{z}}(\theta)} \epsilon^{\frac{x}{c_{1} R_{1} \theta}}\left\{\left(\frac{i w w}{d \xi}\right)-\left(\frac{d w}{d \eta}\right)\right\},
$$


so that, as long as the temperature is constant, the ratio of the quantity of water formed to the quantity of carbonic acid always bears a constant ratio to the ratio of the quantity of free hydrogen to that of free carbonic oxide. This was the result obtained by Horstmann in the experiments before mentioned. 


\section{CHAPTER XV.}

EFFECTS PRODUCED BY ALTERATIONS IN THE PHYSICAL CONDITIONS ON THE COEFFICIENT OF CHEMICAL COMBINATION.

1 20. SINCE the value of

$$
\frac{\epsilon^{a} \zeta^{c}}{\xi^{a} \eta^{b}}
$$

is independent of the values of $\xi, \eta, \zeta, \epsilon$, and since when it is known the amount of chemical combination can be determined, it is convenient to have a name for it, we shall therefore call it the coefficient of chemical combination for $A$ and $B$ and denote it by $k$. The more intense the chemical action between $A$ and $B$ the smaller the values of $\xi, \eta$ in the state of equilibrium and therefore the larger the value of $k$.

We have by equation (220)

$$
k=\phi_{1}(\theta) e^{-\frac{a}{R_{1} \theta} \frac{d Q}{d \xi}} e^{\frac{a}{R_{1} \theta} \frac{d w}{d \xi}}
$$

The alterations which we shall suppose to take place in the physical conditions can be represented by changes $\delta Q$ and $\delta z$ in the values of $Q$ and $w$, and we see from 
equation (226) that if $\delta k$ be the corresponding change in $k$

$$
\frac{\delta k}{k}=-\frac{a}{R_{1} \theta} \frac{d \delta Q}{d \xi}+\frac{a}{R_{1} \theta} \frac{d \delta w}{d \xi} \ldots \ldots \ldots \ldots(227) .
$$

If the substances with which we have to deal are gases we must put $Q$ and $\delta Q$ equal to zero. We considered when we were discussing dissociation in chapter XIV. most of the changes in the physical conditions which could influence the state of chemical equilibrium in this case, and the results obtained then will apply to the more general problem we are discussing now. We see from (227) that any cause producing a change in the potential energy which increases as any chemical action goes on will tend to stop this action which will not have to go on so far before attaining equilibrium as it would if the disturbing cause had been absent and vice versâ.

We shall now go on to consider more particularly the cases of dilute solutions and the effects produced upon chemical equilibrium by changes in the properties of the solvent arising from the progress of chemical change.

I21. Effect of Surface Tension. The first effect we shall consider is that due to the surface tension of the solution. We know that the surface tension depends upon the strength and the nature of the solution, so that since the composition changes as chemical action goes on the surface tension of the solvent and therefore its mean Lagrangian function will change; and therefore by the principle we have just stated the conditions for equilibrium will be altered by the surface tension.

Let $A$ be the area of the surface of the solution, $T$ the surface tension, then the potential energy due to the surface 
tension is $T A$ and there is therefore in the expression for the mean Lagrangian function the term $-T A$, so that by equation (227) the effect of the surface tension on the coefficient of chemical combination is given by the equation

$$
\frac{\delta k}{k}=\frac{a}{R_{1} \theta} \frac{d}{d \xi}(A T) .
$$

Let us endeavour to get some idea of the magnitude of this effect. If $c$ is the molecular weight of the substance whose mass is $\xi$, then since at $0^{\circ} \mathrm{C}$.

$$
\frac{1}{2} c R_{1} \theta=\mathrm{I} \cdot \mathrm{I} \times \mathrm{IO}^{10},
$$

we have, if for simplicity $a$ be put equal to unity,

$$
\begin{aligned}
& \frac{\mathrm{I}}{R_{1} \theta} \frac{d}{d \xi}(A T) \\
= & \frac{c}{2^{2} 2 \times 10^{10}} \frac{d}{d \xi}(A T) .
\end{aligned}
$$

Now $c d(A T) / d \xi$ is the increase in $A T$ when the quantity $\xi$ in the soiution is increased by one gramme-equivalent. If $v$ be the volume of the vessel whose surface we shall suppose to remain constant as combination goes on, then

$$
c \frac{d}{d \xi}(A T)=10^{3} T^{\prime} \frac{A}{v},
$$

where $T^{\prime}$ is the increase in $T$ when the quantity $\xi$ is increased by one gramme-equivalent per litre. Now the experiments of Röntgen and Schneider ("Oberflächen Spannurig von Flüssigkeiten," Wied. Ann. xxix. I65) show that $T^{\prime}$ even in the case of simple salts may be as much as 5 or 6 so that

$$
\frac{\delta k}{k} \text { is of the order } \frac{3}{10^{7}} \frac{A}{v},
$$

and if the solution be spread out in a film of thickness $t$, 
$A / v=2 / t$ so that

$$
\frac{\delta k}{k} \text { is of the order } \frac{6}{10^{7}} \frac{1}{t} \text {; }
$$

thus if the thickness of the film is $1 / 10000$ of a centimetre the value of $k$ is altered by about 6 per cent. If the thickness of the layer is comparable with molecular distance, say about $10^{-\tau}$, then $\delta k / k$ might be as large as 6 . This of course implies that the conditions of equilibrium would be completely altered. 'Thus in very thin films the influence of capillarity might be sufficient to modify completely the nature of chemical equilibrium, though we should not expect it to do much in the body of a fluid.

If the surface tension increases as the chemical action goes on the capillarity will tend to stop the action, while if the surface tension diminishes as the action goes on, the capillarity will tend to increase the action.

Thus the chemical action in a space such as a thin film throughout which the forces producing capillary phenomena are active might be very different from the chemical action in the same substance in bulk when most of it would be free from the action of such forces.

This point does not seem to have received as much attention as it deserves, but there are some phenomena which seem to point to the existence of such an effect. One of these is that called by its discoverer Liebreich " the dead space in chemical reactions," which is well illustrated by the behaviour of an alkaline solution of chloral hydrate. If the proportion of alkali to chloral is properly adjusted, chloroform is slowly deposited as a white precipitate, and if this solution is placed in a test-tube, then at the top of the liquid there is a thin film which remains quite clear and free from chloroform, showing that, unless this effect is due to 
some chemical action of the air, the alkali and chloral do not combine, or if they do chloroform is not precipitated. In fine capillary tubes too, no deposit seems to be formed. This phenomenon could be explained on the above principles if the surface tension of the alkaline solution increases when the alkali combines with the chloral and chloroform is deposited, for in this case the surface tension would increase as chemical action went on, and would therefore tend to stop this action. Dr Monckman made some experiments in the Cavendish Laboratory on the changes in the surface tension of the solution as the reaction went on, and he found that it increased to a very considerable extent, so that this case is in accordance with our theory. The thickness of the dead space (from I to $2 \mathrm{~mm}$. in Liebreich's experiments) is somewhat greater than we should have expected, but any want of uniformity in the liquid such as that produced by the deposition of chloroform itself would increase the thickness of the dead space.

Some other effects produced by surface tension are discussed by Prof. Liveing in his paper "On the Influence of Capillary Action in some Chemical Decompositions" (Proceedings Camb. Phil. Soc. vi. p. 66).

122. Effects due to pressure. Pressure can produce effects of two kinds upon chemical action. The first is when the volume of the liquid under pressure alters as chemical action goes on, the effect of pressure in this case is proportional to the amount of the pressure: the second effect is when the coefficient of compressibility of the liquid changes as the chemical action goes on, the effect of pressure due to this cause is proportional to the square of the pressure.

Let us suppose that $P$ is the external pressure, $r$ 
the volume, we may regard the external pressure as produced by an external system whose mean Lagrangian function is

$$
-P v
$$

and we have by equation (227)

$$
\begin{aligned}
\frac{\delta k}{k} & =\frac{a}{R_{1} \theta} \frac{d}{d \dot{\xi}}(P v) \\
& =\frac{a}{R_{1} \theta} P \frac{d v}{d \xi} \ldots \ldots \ldots \ldots \ldots \ldots . . . . .(228) .
\end{aligned}
$$

Thus if $v$ increases with $\xi, \delta k$ is positive, in other words the value of $\zeta^{c} \epsilon^{d} / \xi^{a} \eta^{b}$ is increased and therefore $\xi$ and $\eta$ are less than they would be if there were no external pressure. Thus the external pressure tends to stop that action which is accompanied by an increase in volume, and vice versâ.

Let us now endeavour to form some estimate of the probable size of this effect. If the molecules of the substance produce the same pressure as if they were in the gaseous state, then at $0^{\circ} \mathrm{C}$.

$$
\frac{1}{R_{1} \theta}=\frac{c}{2: 2 \times 10^{10}}
$$

where $c$ is the combining weight of the substance. Thus if the volume increases by $y$ cubic centimetres per gramme of $A$ formed we have by (228) if the pressure is $x$ atmospheres,

$$
\frac{\delta k}{k}=\frac{c x y a}{2 \cdot 2 \times 10^{4}} .
$$

The cases in which in general $y$ will have the greatest value are those in which we have some of the bodies in solution while others are precipitated, if we suppose that when a salt is dissolved the volume of the solvent is not altered then $y$ will in general be not greatly different from unity, and in this case we have 


$$
\frac{\delta k}{k}=\frac{c a x}{2.2 \times 10^{4}},
$$

so that it would require a pressure of $220 / a c$ atmospheres to change the coefficient of combination by one per cent., thus if the substances taking part in the reaction have large combining weights, the reaction will be sensitive to the influence of pressure.

Let us now consider the effect on the chemical equilibrium when the coefficient of compressibility changes as the chemical action goes on.

Let $\sigma$ be the expansion or contraction of the solution, $\kappa$ its bulk-modulus, $v^{\prime}$ its volume, then in the expression for the potential energy of the solvent there is the term

$$
\frac{1}{2} \gamma^{\prime} \sigma^{2} \kappa \text {, }
$$

and therefore in the expression for the mean Lagrangian function the term

$$
-\frac{1}{2} v^{\prime} \sigma^{2} \kappa
$$

If $\delta k$ be the change in the coefficient of combination due to the change in $\kappa$ as the chemical action progresses we have by equation (227)

$$
\frac{\delta k}{k}=\frac{a}{2 R_{1} \theta} v^{\prime} \sigma^{2} \frac{d \kappa}{d \xi} .
$$

Now if $P$ be the external pressure

$$
\kappa \sigma=P \text {. }
$$

Substituting for $\sigma$ the value given by this equation we get

$$
\frac{\delta k}{k}=\frac{a \eta^{\prime}}{2 R_{1} \theta} \frac{p^{2}}{\kappa^{2}} \frac{d \kappa}{d \xi}
$$

To get some idea of the magnitude of this effect let us suppose that when the mass of $A$ in the solution is increased by one gramme-equivalent per litre the value of $\kappa$ is increased in the proportion of $y$ to $\mathbf{r}$. 
Then

$$
\frac{\mathrm{I}}{\kappa} \frac{d \kappa}{d \xi}=\frac{y \times 10^{3}}{v \times c},
$$

where $c$ is the molecular weight of $\xi$, we have therefore by equation (229)

$$
\frac{\delta k}{k}=\frac{a y}{2 R_{1} c \theta} \frac{P^{2}}{\kappa} \times r o^{3} .
$$

Now

$$
c R_{1} \theta=2 \cdot 2 \times 10^{10},
$$

and for water,

$$
\kappa=2.2 \times 10^{10} .
$$

So that if the pressure is $x$ atmospheres

$$
\frac{\delta k}{k}=\frac{a x^{2} y}{10^{6}} \text { approximately. }
$$

From the results of Röntgen and Schneider's experiments given in $\$(97)$ we see that $y$ will often be as large as $1 / 10$, so that in this case, supposing $a$ unity, the effect of a pressure of Io0 atmospheres would be to alter $k$ by I/ro per cent. while a pressure of rooo atmospheres would alter it by ro per cent.

If the bulk-modulus increases as $\xi$ increases then the action of the pressure is to retard the chemical action by which $\xi$ increases.

123. Effect of magnetism on chemical action. The magnetic properties of solutions are generally so feeble that we cannot expect magnetism to produce any effect except upon those which contain iron. In some of the chemical actions however in which iron is dissolved or deposited magnetism does seem to affect the result. Thus when a solution of copper sulphate is placed on an iron plate copper is deposited and iron dissolved, and if this plate be placed over the poles of a powerful electro-magnet it is found that the copper deposit is thinnest over the poles, the places where the magnetic force is the most powerful. 
The effect of the magnetic force is easily found. Let $I$ be the intensity of magnetization of the solution, $I^{\prime}$ that of the iron plate, $H$ and $H^{\prime}$ the magnetic forces and $k^{\prime}$ and $k^{\prime \prime}$ the coefficients of magnetization of the solution and iron plate respectively, $v$ and $v^{\prime}$ the volumes of these substances, then if $k^{\prime}$ and $k^{\prime \prime}$ are constant there is by $\S 34$ the term

$$
-\frac{1}{2}\left\{\frac{I^{2}}{k^{\prime}} v+\frac{I^{\prime 2}}{k^{\prime \prime}} v^{\prime}\right\}+H I v+H^{\prime} I^{\prime} z^{\prime}
$$

in the expression for the mean Lagrangian function.

Thus we have by equation (227) .

$$
\frac{\delta k}{k}=\frac{a}{2 R_{1} \theta}\left\{-\frac{I^{2}}{k^{\prime 2}} \frac{d k^{\prime}}{d \xi} v-\frac{I^{\prime 2}}{k^{\prime \prime} \sigma}+\frac{2 H^{\prime} I^{\prime}}{\sigma}\right\},
$$

where $\sigma$ is the density of the iron, and $\xi$ the quantity of iron in the solution.

Since

$$
\begin{gathered}
H=k^{\prime} I, \\
H^{\prime}=k^{\prime \prime} I^{\prime},
\end{gathered}
$$

we get

$$
\frac{\delta k}{k}=\frac{a}{2 R_{1} \theta}\left(\frac{I^{\prime 2}}{k^{\prime \prime} \sigma}-\frac{I^{2}}{k^{\prime 2}} \frac{d k^{\prime}}{d \xi} v\right) .
$$

Since in practice $I^{\prime 2} / k^{\prime \prime} \sigma$ is greater than $I^{2} \frac{d k^{\prime}}{d \xi} v / k^{\prime}$ we see that $\delta k$ will be positive and will increase with $l^{\prime}$, hence since $k=\zeta^{c} \epsilon^{i} / \xi^{a} \eta^{b}$, the quantity of iron dissolved will be least where $I^{\prime}$ is greatest, that is where the magnetic field is strongest, which agrees with the results of experiment.

We can show in a similar way that any chemical action which produces an increase in the coefficient of magnetization is hindered by the action of magnetic forces.

If we place a solution of an iron salt in a magnetic field where the strength is not uniform the magnetic force will cause the strength of the solution to be greater in those 
parts of the field where the force is intense than in those where it is weak.

To calculate the magnitude of the effect due to this cause let us suppose that the solution is contained in two vessels connected with each other by a tube of small bore, and that one vessel is placed in a region where the magnetic force vanishes, the other in one where it is constant and equal to $H$. Then if $\xi$ and $\eta$ are the number of molecules of the salt in unit volume of the first and second of these vessels respectively, we can easily prove by equating to zero the variation of the mean Lagrangian function for the liquid in the two vessels that

$$
R_{1} \theta \log \frac{\xi}{\eta}=\frac{1}{2} \frac{I^{2}}{k^{\prime 2}} \frac{d k^{\prime}}{d \xi},
$$

where $k^{\prime}$ is the coefficient of magnetization of the solution. Thus if the coefficient of magnetization increases with the strength of the solution the magnetic force will tend to drive the salt from the weak to the strong parts of the magnetic field. 


\section{CHAPTER XVI.}

CHANGE OF STATE FROM SOLID TO LIQUID.

I24. THE cases we have hitherto considered have been those in which gases and dilute solutions have been chiefly concerned, in this chapter we shall consider the phenomena of solution, fusion and solidification in which liquids and solids play the chief part.

\section{Solution.}

125. Let us consider the case of a mixture of salt and solvent in equilibrium, and endeavour to find how the amount of salt dissolved depends upon various physical circumstances.

Let $\xi$ be the mass of the salt, $\eta$ that of the solution. Let us for brevity denote $\overline{d p} / d \theta$ for unit volume of the salt by $\omega$ and the corresponding quantity for the solution by $\omega^{\prime}$. Let $w_{1}, w_{2}$ be the potential energies of unit masses of the salt and solution respectively.

Then the mean Lagrangian function for the salt is

$$
\theta \int \omega d v+\xi f_{1}(\theta)-\xi w_{1},
$$


where $f_{1}(\theta)$ is the part of the mean kinetic energy of unit mass which does not depend upon the controllable coordinates.

If $v$ be the volume of the salt and we put

$$
\int \omega d v=\Omega v \text {, }
$$

then the Lagrangian function for the salt may be written

$$
\theta \Omega v+\xi f_{1}(\theta)-\xi w_{1} .
$$

The mean Lagrangian function for the solution is with a similar notation

$$
\theta \Omega^{\prime} v^{\prime}+\eta f_{2}(\theta)-\eta z v_{2}
$$

where $f_{2}(\theta)$ is the part of the kinetic energy of unit mass of the liquid which does not depend upon the controllable coordinates, and $z^{\prime}$ is the volume of the solution. We must remember that though $\Omega$ and $w_{1}$ do not depend upon the values of $\xi$ and $\eta$ yet the values of $\Omega^{\prime}, w_{2}$ and $f_{2}(\theta)$ may do so as the properties of the solution may and generally do alter when the amount of salt the solution contains is altered.

By the Hamiltonian principle the value of the mean Lagrangian function of the salt and solution when in equilibrium is stationary.

Let us suppose that when the system is in equilibrium the conditions are disturbed by a mass $\delta \xi$ of the salt melting, then the change in the value of $H$ is, if $\sigma$ be the density of the salt, $\rho$ that of the solution,

$$
\begin{aligned}
\left\{-\theta \frac{\Omega}{\sigma}-f_{1}(\theta)+w_{1}+\theta\right. & \frac{\Omega^{\prime}}{\rho}+\theta \frac{d \Omega^{\prime}}{d \eta} v^{\prime} \\
& \left.+f_{2}(\theta)+\eta \frac{d f_{2}(\theta)}{d \eta}-w_{2}-\eta \frac{d w_{g}}{d \eta}\right\} \delta \xi .
\end{aligned}
$$

Since the value of $H$ is stationary this quantity must 
vanish when there is equilibrium so that we get

$\frac{\Omega^{\prime}}{\rho}+\frac{d \Omega^{\prime}}{d \eta} v^{\prime}+\frac{\mathrm{I}}{\theta} \frac{d}{d \eta} \cdot \eta f_{2}(\theta)-\frac{\Omega}{\sigma}-\frac{f_{1}(\theta)}{\theta}=\frac{w_{2}+\eta \frac{d w_{2}}{d \eta}-w_{1}}{\theta} \ldots(230) ;$

if we knew how the quantities in this equation varied with the amount of salt dissolved we could use it to determine the amount of salt dissolved when the solution is saturated. But though we have not this knowledge and therefore cannot use this equation to determine the solubility of a salt in a given solvent, we can still get a good deal of information from it about the effect produced by various physical circumstances on the solubility.

126. The first effect of this kind we shall consider is that of pressure; and, just as in the case of chemical combination, pressure will produce two effects, one depending on the change of volume which takes place on solution, the other on the change produced in the coefficient of compressibility.

Let us consider first the effect due to the change in volume.

We may suppose that the external pressure is produced by a weight placed on a piston which presses on the fluid, the mean Lagrangian function of this system is

$$
-p V
$$

where $V$ is the volume of the salt and solution; the increase in this when $\xi$ diminishes by $\delta \xi$ is

$$
\delta \xi p \frac{d V}{d \xi}
$$


so that in this case instead of $(230)$ we have now

$$
\begin{aligned}
\frac{\Omega^{\prime}}{\rho}+\frac{d \Omega^{\prime}}{d \eta} v^{\prime}+\frac{\mathbf{I}}{\theta} \frac{d}{d \eta}\left\{\eta f_{2}(\theta)\right\}-\frac{\Omega}{\sigma}-\frac{\mathbf{I}}{\theta} f_{1}(\theta) & \\
= & \frac{\mathbf{I}}{\theta}\left(w_{2}+\eta \frac{d w_{2}}{d \eta}-w_{1}-p \frac{d V}{d \xi}\right)(23 \mathrm{x}) .
\end{aligned}
$$

We shall endeavour to find the change in temperature which would produce the same effect on the solubility as the pressure $p$.

We may regard the expression

$$
\frac{\Omega^{\prime}}{\rho}+\frac{d \Omega^{\prime}}{d \eta} v^{\prime}+\frac{\mathbf{I}}{\theta} \frac{d}{d \eta}\left\{\eta f_{\mathrm{a}}(\theta)\right\}-\frac{\Omega}{\sigma}-\frac{\mathbf{I}}{\theta} f_{1}(\theta)-\frac{\mathbf{I}}{\theta}\left(w_{2}+\eta \frac{d w_{2}}{d \eta}-w_{1}\right)
$$

as a function of $\xi$, say $f(\xi)$, then if $\theta$ be increased by $\delta \theta$ the corresponding change $\delta \xi$ in $\xi$ is, by equation (230), approximately given by the equation

$$
f^{\prime}(\xi) \delta \xi=-\frac{\mathrm{I}}{\theta^{2}}\left(w_{2}+\eta \frac{d w_{2}}{d \eta}-w_{1}\right) \delta \theta ;
$$

this equation is only approximate as we have neglected the variations of $\Omega, \Omega^{\prime}, f_{1}(\theta) / \theta$ and $f_{\mathrm{a}}(\theta) / \theta$ with the temperature.

If $\delta \xi_{1}$ be the clange produced by the pressure $p$, the temperature remaining constant, we have by equation (23I)

$$
f^{\prime}(\xi) \delta \xi_{1}=-\frac{p}{\theta} \frac{d V}{d \xi},
$$

so that the change $\delta \theta$ in the temperature which would produce the same effect as the pressure $p$ is given by the equation

$$
\underset{\theta^{2}}{\mathbf{I}}\left(w_{2}+\eta \frac{d w_{2}}{d \eta}-w_{1}\right) \delta \theta=\frac{p}{\theta} \frac{d V}{d \xi} \ldots \ldots \ldots \ldots\left(23^{2}\right) .
$$


Now $w_{2}+\eta \frac{d w_{2}^{\prime}}{d \eta}-w_{1}$ is the increase in the potential energy when unit mass of the salt dissolves; this will be measured by $q$ the mechanical equivalent of the heat absorbed in this process at zero temperature, or at any temperaiure, if the specific heat of the system does not change as the salt dissolves: making this substitution equation $\left(23^{2}\right)$ becomes

$$
\delta \theta=p \frac{\theta}{q} \frac{d V}{d \xi} \ldots \ldots \ldots \ldots \ldots \ldots(233) .
$$

If the volume diminishes as the salt dissolves $d V / d \xi$ is positive, so that if $q$ be positive the effect of pressure is the same as that of an increase in temperature, while if the volume increases as the salt dissolves the effect of pressure will be the same as that of a diminution in temperature.

The effect of pressure upon the solubility of various salts has been investigated by Sorby (Proceedings Royal Society, xir. p. 538, I863). The salts he examined were sodium chloride, copper sulphate, and the ferri- and ferrocyanides of potassium. He found that when the volume increased on solution the solubility was diminished by pressure, while when the volume diminished on solution the solubility was increased by the same means. This agrees with the results of equation (233).

The results of his experiments are given in the following table-the first column of which gives the name of the salt dissolved, the second the increase in volume when roo c.c. of the salt crystallizes out, the third the increase in the salt dissolved when a pressure of 100 atmospheres is applied, and the fourth the value of this quantity calculated by (233) 
Sodium Chloride

Copper Sulphate

Potassium Ferricyanide

Potassium Sulphate

Potassium Ferrocyanide

\begin{tabular}{|c|c|c}
13.57 & 419 & 56 \\
4.83 & 3.183 & 2.4 \\
2.51 & 0.335 & $\cdot 28$ \\
$31 \cdot 21$ & $2 \cdot 914$ & 4.4 \\
8.9 & 2.845 &
\end{tabular}

The numbers required to calculate by the aid of (233) the theoretical amount of the alteration in the solubility are given below.

The heat absorbed when the salt dissolves depends upon the strength of the solution and the temperature, the value of $q$ required for our purpose is that which corresponds to a saturated solution at the zero of absolute temperature; as the variations in the value of $q$ with temperature are probably due to changes in the specific heat the effect of these changes will be smaller the lower the temperature, we shall always therefore take the heat of dissolution for the lowest temperature at which it has been observed, though when the variation with temperature is rapid this can only be a very rough approximation.

Sodium chloride.

$q$ at $0^{\circ} \mathrm{C}$. for a strong solution $=\frac{5^{\circ} 6}{5^{8}} \times 4.1 \times 10^{9}$ (Ostwald's Lehrbuch der Allgemeinen Chemie, II. p. 170).

Specific gravity $=2 \cdot I$ (Watts' Dictionary of Chemistry, v. p. 335).

According to Gay-Lussac (Annales de Chemie et de Physique, XI. p. 3ro, I819) the increase in solubility for each degree centigrade is

$$
\frac{4 \cdot 7}{35 \cdot 15}=\cdot 13 \% \text {. }
$$

Sulphate of copper. $\mathrm{CuSO}_{4}+{ }_{5} \mathrm{H}_{2} \mathrm{O}$. 
$q$ at $15^{\circ} \mathrm{C} . ?=\frac{27^{\circ} 2}{249^{\circ} 3} \times 4^{\circ} \mathrm{I} \times 10^{9} \quad \begin{gathered}\text { (Ostwald, Lehrbuch, II. } \\ \text { p. 250). }\end{gathered}$

Specific gravity $=2 \cdot 2$ (Watts' Dictionary of Chemistry, v. 59r).

Increase of salt dissolved for a rise in temperature of $\mathrm{I}^{\circ} \mathrm{C} .=\mathrm{I} \cdot 7^{\circ}$ (Watts' Dictionary of Chemistry, v. $59 \mathrm{I}$ ).

Ferricyanide of Potassium.

$q$ at $15^{\circ} \mathrm{C} . ?=\frac{144}{25^{\mathrm{I}}} \times 4^{.1} \times 109 \quad \begin{aligned} & \text { (Ostwald, Lehrbuch, II. } \\ & \left.\text { p. } 35^{2}\right) .\end{aligned}$

Specific gravity $=\mathrm{I} \cdot 8$ (Watts' Dictionary of Chemistry, II. 247).

Increase of salt dissolved for a rise in temperature of $\mathbf{I}^{\circ} \mathrm{C} .=\mathbf{I} \cdot 27^{\circ} \%$ (Watts' Dictionary, II. 247).

Potassium sulphate.

$q$ at $15^{\circ}$ C. $?=\frac{64}{174} \times 4.1 \times 10^{9}$ (Ostwald, Lehrbuch, I1. p. I62).

Specific gravity $=2.6$ (Watts' Dictionary, v. 607).

Increase of salt dissolved for a rise in temperature of $\mathbf{I}^{\circ}$ C. $=2 \%$ (Gay-Lussac, Annales de Chemie et de Physique, XI. p. 3 I I, I8I9).

I have not been able to find corresponding data for the ferrocyanide of potassium.

As an example of the way in which the effects of pressure can be calculated from these data let us take the case of sodium chloride: since $13.57 / 100$ is the increase in volume when I c.c. of the salt crystallizes out, and $2 \cdot I$ is the specific gravity of the salt,

$$
\frac{d V}{d \xi}=\frac{{ }^{I} 357}{2 \cdot \mathrm{I}} \text {. }
$$


When the pressure is 100 atmospheres and the temperature $15^{\circ} \mathrm{C}$.

$$
\begin{aligned}
& p=10^{8}, \\
& \theta=288,
\end{aligned}
$$

so that by equation (233)

$$
\begin{gathered}
\delta \theta=\frac{288 \times 5^{8} \times 1357 \times 10^{8}}{5^{\circ} 6 \times 4^{\circ} \mathrm{I} \times 2.1 \times 10^{9}} \\
\delta \theta=44^{\circ} \mathrm{C} .,
\end{gathered}
$$

and since the solubility increases $13 \%$ for each degree, the solubility is increased by the pressure by ${ }^{\circ} 6$ parts in roo.

Considering the imperfect nature of the data at our disposal the agreement between the theory and the experiments seems as close as could have been expected.

So far we have neglected the effect of the difference between the compressibility of the salt and the solution, but as this may be very considerable it is necessary to investigate this effect in order to see when it may legitimately be omitted.

If the bulk modulus of the salt is $k$, and that of the solvent $k^{\prime}$, then in the mean Lagrangian function of the two there is the term

$$
-\frac{1}{2} k e^{2} v-\frac{1}{2} k^{\prime} e^{\prime 2} v^{\prime}
$$

where as before $v$ and $v^{\prime}$ are the volumes of the salt and the solution respectively, and $e$ and $e^{\prime}$ their contractions.

Taking this term into account we find that the condition

leads to the equation

$$
\frac{d H}{d \xi}=\circ
$$

$$
\begin{aligned}
& \frac{\Omega^{\prime}}{\rho}+\frac{d \Omega^{\prime}}{d \eta} v^{\prime}-\frac{\Omega}{\sigma}+\frac{\mathbf{I}}{\theta}\left[\frac{d}{d \eta}\left\{\eta f_{2}(\theta)\right\}-f_{1}(\theta)\right]= \\
& \quad \frac{\mathbf{I}}{\theta}\left\{w_{2}+\eta \frac{d w_{2}}{d \eta}-w_{1}-p \frac{d V}{d \xi}-\frac{\mathbf{I}}{2} \frac{p^{2}}{k} \frac{\mathbf{I}}{\sigma}-\frac{\mathbf{I}}{2} \frac{p^{2}}{k^{\prime 2}} \frac{d}{d \xi} k^{\prime} v^{\prime}\right\},
\end{aligned}
$$


and if $\delta \theta$ is the increase in temperature which would produce the same effect as the pressure

$$
\delta \theta=\frac{\theta}{q}\left\{p \frac{d V}{d \xi}+\frac{\mathrm{I}}{2} \frac{p^{2}}{k} \frac{\mathrm{I}}{\sigma}+\frac{\mathrm{I}}{2} \frac{p^{2}}{k^{\prime 2}} \frac{d}{d \xi}\left(k^{\prime} v^{\prime}\right)\right\} .
$$

Since $k^{\prime}$ is of the order $10^{10}$, we see that if the change which takes place in the volume of the salt when it dissolves amounts to one per cent. of its original volume the terms involving $p^{2}$ are not so important as those involving $p$ if the pressure is not more than roo atmospheres. For very much larger pressures however the terms depending upon $p^{2}$ will be the most important, and in this case the effect of the pressure will be proportional to the square of the pressure and not to its first power, as in the cases examined by Sorby.

I27. Effect of Surface Tension upon the Solubility. Surface tension may affect the amount of salt required to saturate a solution in several ways.

In the first place the surface tension of the solution may change as the salt dissolves; secondly, the alteration which takes place in the volume may change the area of the surface in contact with the glass or the air, and again when the salt dissolves or is deposited the surface of contact of the salt and solution may change; when the salt is precipitated as a fine powder this increase in surface may be very considerable.

To find the effect of these changes on the solubility, let $S$ be a surface of the solvent, $T$ its surface tension. Then in the expression for the mean Lagrangian function of the solvent there is the term

$$
-\Sigma T S
$$

where the summation is extended over all the surfaces of the solvent. 
Then we get by applying the same methods as before,

$$
\begin{aligned}
\frac{\Omega^{\prime}}{\rho}+\frac{d \Omega^{\prime}}{d \eta} v^{\prime}-\frac{\Omega}{\sigma}+\frac{\mathbf{I}}{\theta}\left[\frac{d}{d \eta}\left\{\eta f_{2}(\theta)\right\}\right. & \left.-f_{1}(\theta)\right] \\
& =\frac{w_{2}+\eta \frac{d w_{2}}{d \eta}-w_{1}}{\theta}-\frac{\mathbf{I}}{\theta} \frac{d}{d \xi} \Sigma T S ;
\end{aligned}
$$

proceeding as in $\$ 126$ we see that the increase $\delta \theta$ in the temperature which would produce the same effect as the surface tension is given by the equation

$$
\delta \theta=\frac{\theta}{q} \Sigma \frac{d}{d \xi}(T S)
$$

so that if $T S$ increases as the salt dissolves the effect of the surface tension will be to retard solution, while it will increase the solubility if TS diminishes.

Let us take as an example the case when the fluid is in spherical drops and consider the effect of the change in volume which takes place as the salt dissolves. If $a$ is the radius of the drop and $i$ the increase in volume when unit mass of the salt dissolves, then

$$
\begin{aligned}
i & =-\frac{d}{d \xi}\left(\frac{4}{3} \pi a^{3}\right) \\
& =-4 \pi a^{2} \frac{d a}{d \xi},
\end{aligned}
$$

so that if $S$ be the surface

$$
\begin{aligned}
\frac{d S}{d \xi} & =8 \pi a \frac{d a}{d \xi} \\
& =-\frac{2 i}{a}
\end{aligned}
$$

and therefore by equation (234)

$$
\delta \theta=-2 i \frac{\theta T}{q a} .
$$


Let us take the case of potassium sulphate, for which $i=\mathrm{I} / \mathrm{I} 2$ and $q=\mathrm{I}^{\circ} 5 \times 10^{9}$,

$$
\frac{\delta \theta}{\theta}=\frac{2 T}{a} \frac{\mathrm{I}}{\mathrm{I} \cdot 8 \times 10^{10}} ;
$$

since $T$ is about $8 \mathrm{I}$, we have at the temperature of $27^{\circ} \mathrm{C}$.

$$
\delta \theta=\frac{3}{10^{6}} \frac{\mathbf{I}}{a} \text { approximately, }
$$

so that if the radius of the drops was $1 / 10000$ of a millimetre

$$
\delta \theta=\frac{3}{10}
$$

and since the solubility increases by $2 \%$ for each degree of temperature the solubility of spray of this fineness would be diminished by about $6 \%$.

In this case the effect of the surface tension is very small, but if the salt were deposited from its solution as a very fine powder the effect of the increase in the surface might be much more considerable.

Let us suppose that the salt is deposited in the shape of small spheres of radius $a$, then

$$
\frac{d S}{d \xi}=\frac{3}{\sigma a},
$$

and if $T^{\prime}$ be the surface tension of the salt and solution we shall have

$$
\frac{\delta \theta}{\theta}=\frac{3}{\sigma a} \frac{T^{\prime}}{q}
$$

in some cases the particles in which the salt is deposited are fine enough to scatter light, so that their diameter must be much less than the wave length of the blue rays, we may 
therefore put $a=10^{-6}$; we do not know the value of $T^{\prime}$ but it is probably greater than $T$ for the surface of contact of air and the solution; even though it were no greater we should have with these numbers for potassium sulphate

$$
\frac{\delta \theta}{\theta}=\frac{\mathrm{I}}{\mathrm{IO}} \text { approximately, }
$$

so that at $27^{\circ} \mathrm{C}$. the solubility would be changed by about 60 per cent. This effect would help the salt to dissolve and prevent its deposition from the solution. If the salt before solution was not in a very finely divided condition the diminution in the surface caused by the solution would be much less than the increase in the surface due to the deposition of the salt, so that surface tension would be much more efficacious in preventing deposition from the solution than in helping the salt to dissolve, it would thus tend to promote something analogous to super-saturation.

Let us now consider the effect due to the alteration in the surface tension of the solution with the quantity of salt dissolved. We have as before

$$
\frac{\delta \theta}{\theta}=\frac{S}{q} \frac{d T}{d \xi} \ldots \ldots \ldots \ldots \ldots \ldots(235) .
$$

According to Röntgen and Schneider (Wied. Annalen, xxix. p. 209, I 886) the surface tension of an $8 \%$ solution of potassium sulphate is about $3 \%$ greater than that of pure water, for this substance we have therefore, approximately,

$$
\frac{d T}{d \hat{\xi}}=-\frac{8 \mathrm{I} \times 3}{8 v}
$$

where $v$ is the volume of solution; substituting this value for $d T / d \xi$ in equation (235) and putting $q=1.5 \times 10^{9}$, 


$$
\begin{aligned}
\frac{\delta \theta}{\theta} & =-\frac{8 \mathrm{I} \times 3}{8 \times 15 \times 10^{9}} \times \frac{S}{v} \\
& =-\frac{2}{10^{8}} \frac{S}{v} \text { approximately. }
\end{aligned}
$$

If the solution is in a cylindrical tube of radius $a, S / v=2 / a$, and therefore

$$
\frac{\delta \theta}{\theta}=\frac{4}{10^{8}} a \text { approximately. }
$$

The sign is changed because if the angle of contact vanishes the increase in the surface tension of the surface separating the solution and air is equal to the diminution in that separating the solution and the walls of the tube. If we suppose that these cylindrical tubes are of the dimensions of the pores in such substances as meerschaum or graphite, then since we know by the laws of diffusion of gases through these substances that the diameter of the pores must be comparable with the mean free path of a molecule of the gas we may assume that $a$ is of the order $10^{-6}$. In this case

$$
\frac{\delta \theta}{\theta}=\frac{4}{100},
$$

so that at $27^{\circ} \mathrm{C}$. the value of $\delta \theta$ would be about $12^{\circ} \mathrm{C}$., which in the case of potassium sulphate is equivalent to an increase in the solubility by nearly $25 \%$. In most cases the surface tension of the surface separating a solution from air increases with the amount of salt in it, so that the salt will be more soluble in liquid in capillary spaces than in liquid in bulk.

\section{Liquefaction.}

I28. Under this head we shall consider the influence of changes in the physical condition on the passage of a substance from the solid to the liquid state and vice versâ. 
This problem has much in common with that of solution, but since in this case the liquid and solid are the same substances in different states, the properties of the liquid will not, as in that case, change as the solid melts.

Let $\xi$ be the mass of the solid, by $\S 8 \mathrm{r}$ its mean Lagrangian function is

$$
\theta \int \frac{\overline{d p}}{d \theta} d v+\xi f_{1}(\theta)-\xi w_{1}
$$

where $w_{1}$ is the potential energy of unit mass of the substance in the solid state.

Since the Lagrangian function is proportional to the volume, we may put

$$
\theta \int \frac{\overline{d p}}{d \theta} d v=\theta z \Omega
$$

where $v$ is the volume of the solid.

If $\eta_{1}$ is the mass of the liquid, $v^{\prime}$ its volume, $w_{2}$ the potential energy of unit mass, the Lagrangian function of the liquid is

$$
\theta v^{\prime} \Omega^{\prime}+\eta f_{\mathbf{z}}(\theta)-\eta w_{2},
$$

where $\Omega^{\prime}$ is defined by the equation

$$
\theta \int^{\overline{d p}} \frac{\bar{d} \theta}{\theta} d v=\theta v^{\prime} \Omega^{\prime}
$$

The terms $f_{1}(\theta), f_{2}(\theta)$ are the parts of the Lagrangian function which clo not depend upon strain \&c., that is, they do not involve the controllable coordinates. They are therefore independent of the arrangement of the molecules and depend merely upon the number of the molecules and the kinetic energy possessed by each. We should therefore expect that so long as the temperature remains constant these terms would not alter much however the arrangement of the molecules might change, provided the molecules were not decomposed. 
If there is no external pressure the change in the mean Lagrangian function of the solid and liquid when the mass $\delta \xi$ of the liquid freezes is

$$
\delta \xi\left\{\theta\left(\frac{\Omega}{\sigma}-\frac{\Omega^{\prime}}{\rho}\right)+f_{1}(\theta)-f_{2}(\theta)-\left(w_{1}-w_{8}\right)\right\},
$$

where $\sigma$ and $\rho$ are the densities of the solid and liquicl respectively.

This change must vanish by the Hamiltonian principle when the system is in equilibrium, so that in this case we have

$$
\theta\left\{\frac{\Omega}{\sigma}-\frac{\Omega^{\prime}}{\rho}\right\}+f_{1}(\theta)-f_{2}(\theta)-\left(w_{1}-w_{2}\right)=0 . . .\left(23^{6}\right) .
$$

We may regard the left-hand side of this equation as a function of $\theta$, say $\phi(\theta)$, which when equated to zero gives $\theta$ the temperature at which melting takes place.

Let us now consider the effect of a slight change in the physical conditions. If this change increases the Lagrangian function by $\chi$ and does not affect appreciably the values of $\Omega / \sigma, \Omega^{\prime} / \rho$, we have if the melting point is now $\theta+\delta \theta$,

$$
\phi(\theta+\delta \theta)=-\frac{d \chi}{d \xi},
$$

or since $\phi(\theta)=0$

$$
\delta \theta \frac{d}{d \theta} \phi(\theta)=-\frac{d \chi}{d \xi} \ldots \ldots \ldots \ldots . . .(237) .
$$

Let us consider the effect of pressure upon the freezing point. If the external pressure is $p$ then

and since

$$
x=-p\left(v+v^{\prime}\right)
$$

$$
\begin{aligned}
& \frac{d}{d \xi}\left(v+v^{\prime}\right)=\frac{\mathbf{I}}{\sigma}-\frac{\mathbf{I}}{\rho}, \\
& \frac{d \chi}{d \xi}=-p\left(\frac{\mathbf{I}}{\sigma}-\frac{\mathbf{I}}{\rho}\right)
\end{aligned}
$$

T. D. 
so that

$$
\delta \theta \frac{d \phi(\theta)}{d \theta}=p\left(\frac{\mathbf{I}}{\sigma}-\frac{\mathbf{I}}{\rho}\right) .
$$

But from equation (I 16 ) we have

$$
\delta Q=\theta \delta v\left(\frac{d p}{d \theta}\right)_{v \text { constant }},
$$

and if the heat supplied is just sufficient to melt unit mass of ice, $\delta Q=\lambda$, the latent heat of liquefaction and $\delta v=r / \rho-1 / \sigma$, hence

$$
\lambda=\theta\left(\frac{d p}{d \theta}\right)\left(\frac{\mathrm{I}}{\rho}-\frac{\mathrm{r}}{\sigma}\right),
$$

whence if $\delta \theta$ be the increase in $\theta$ caused by the pressure $p$

$$
\delta \theta=p \frac{\theta}{\lambda}\left(\frac{\mathrm{I}}{\rho}-\frac{\mathbf{I}}{\sigma}\right) \ldots \ldots \ldots \ldots \ldots\left(23^{8}\right) ;
$$

comparing this with (237) we see that

$$
\frac{d \phi(\theta)}{d \theta}=-\frac{\lambda}{\theta}
$$

and equation (237) becomes

$$
\delta \theta=\frac{\theta}{\lambda} d \chi
$$

So that if the Lagrangian function increases when the liquid freezes, the temperature at which freezing takes place is raised, in other words freezing is facilitated. This is another example of the principle of $\$ 84$.

We see from equation $\left(23^{8)}\right.$ that if the body expands on solidification $\delta \theta$ is negative or the melting point is lowered by pressure, if the body contracts on solidification $\delta \theta$ is positive and the melting point is raised by pressure. This is the well-known effect predicted by Prof. James Thomson and verified by the experiments of Sir William Thomson.

This however is not the only effect produced by pressure 
on the melting point, there is another effect arising from the difference between the energy due to strains produced by the pressure in unit mass before and after solidification. This energy is proportional to the square of the pressure, so that the lowering of the freezing point from this cause will also be proportional to the square of the pressure.

Let as before $p$ be the pressure per unit area acting on the solid and liquid, let $k$ be the modulus of compression of the solid, $k^{\prime}$ that of the liquid, the potential energy due to the strain in the solid and liquid is

$$
\frac{1}{2} v \frac{p^{2}}{k}+\frac{1}{2} v^{\prime} \frac{p^{2}}{k^{\prime}}+p\left(v+v^{\prime}\right),
$$

so that in the mean Lagrangian function of the solid and liquid there is the term

$$
-p\left\{v_{0}(\mathbf{I}-\delta)+v_{0}^{\prime}\left(\mathrm{I}-\delta^{\prime}\right)\right\}-\frac{1}{2} v_{0} k \delta^{2}-\frac{1}{2} v_{0}^{\prime} k^{\prime} \delta^{\prime 2},
$$

where $\delta$ and $\delta^{\prime}$ are the compressions and $v_{0}$ and $z_{0}^{\prime}$ the volumes, $\sigma_{0}$ and $\rho_{0}$ the densities of the solid and liquid when free from pressure.

If $\delta \theta$ be the rise in the melting point due to this cause we see from (239) that

$$
\begin{aligned}
\frac{\delta \theta}{\theta} & =\frac{\mathbf{I}}{\lambda}\left(\frac{p \delta}{\sigma_{0}}-\frac{p \delta^{\prime}}{\rho_{0}}-\frac{\mathbf{I}}{2} \frac{k \delta^{2}}{\sigma_{0}}+\frac{\mathrm{I}}{2} \frac{k^{\prime} \delta^{\prime 2}}{\rho_{0}}\right) \\
& =\frac{p^{2}}{2 \lambda}\left(\frac{\mathrm{I}}{k \sigma_{0}}-\frac{\mathrm{I}}{k^{\prime} \rho_{0}}\right) .
\end{aligned}
$$

So that unless $k \sigma_{0}=k^{\prime} \rho_{0}$ the freezing point will be altered by an amount proportional to the square of the pressure.

Let us find the magnitude of this effect in the case of ice and water. The only constant of elasticity for ice which has been determined is Young's modulus, which Bevan determined by flexure experiments to be about $6 \times 10^{10}$, the modulus of compression $k$ is therefore not likely to be less 
than $4 \times 10^{10}$. The value of this quantity for water is about $2 \times 10^{10}$. Substituting these values we get

$$
\frac{\delta \theta}{\theta}=-\frac{\mathrm{I}}{8 \lambda} p^{\mathrm{g}} \mathrm{ro}^{-10},
$$

roughly.

This acts in the same direction as the effect due to the alteration in volume on solidification. Comparing this expression with equation $(238)$ we see that for pressures less than about 9000 atmospheres the effect depending on the change in volume is the more important, while for pressures greater than this the effect we have just been investigating is the larger. If $k \sigma$ is greater than $k^{\prime} \rho$ then this effect in the case of substances which contract when they solidify is in the opposite direction to that which is proportional to the first power of the pressure, so that in these cases the effect of pressure upon the freezing point is reversed when the pressure exceeds a critical value.

129. Effect of torsion upon the freezing point. Let us suppose that we have a cylindrical bar of ice twisted with a uniform twist about its axis; it will possess energy in virtue of the strain, but if it melts (suppose on the - outside) the water will be free from strain, and will not therefore possess any energy corresponding to that possessed by the twisted ice. Thus the potential energy will diminish, and the Lagrangian function therefore increase as the ice melts, so that by the principle stated in $\$ 84$ the torsion will facilitate the melting of the ice, that is, it will lower the freezing point.

We can easily calculate the magnitude of this effect. Let us take the case of a thin cylindrical tube of ice, since in this case the strain is uniform, and let $a$ and $b$ be respectively the external and internal radii of the tube, 
$l$ its length, $n$ the coefficient of rigidity of ice, $\phi$ the uniform twist produced by a force $P$ acting at an $\operatorname{arm} b$, then in the mean Lagrangian function of the tube there are the terms

$$
\begin{aligned}
& P b l \phi-\frac{\pi}{4} l \phi^{2} n\left(a^{4}-b^{4}\right) \\
& =P b l \phi-\frac{1}{4} \phi^{2} n v\left(a^{2}+b^{2}\right),
\end{aligned}
$$

where $v$ is the volume of the ice.

So that if $\delta \theta$ be the rise in the freezing point produced by the torsion we have

$$
\begin{aligned}
\frac{\delta \theta}{\theta} & =\frac{\mathrm{I}}{\lambda} \frac{d}{d \xi}\left\{P b l \phi-\frac{1}{4} \phi^{2} n v\left(a^{2}+b^{2}\right)\right\} \\
& =\frac{\mathrm{I}}{\lambda}\left\{\left[P b l-\frac{1}{2} \phi n v\left(a^{2}+b^{2}\right)\right] \frac{d \phi}{d \xi}-\frac{1}{4} \phi^{2} n \frac{d}{d \xi} v\left(a^{2}+b^{2}\right)\right\} \\
& =-\frac{\mathrm{I}}{4 \lambda} \phi^{2} n \frac{d}{d \xi}\left\{v\left(a^{2}+b^{2}\right)\right\} .
\end{aligned}
$$

If the sides melt equally we have since $a$ and $b$ are approximately equal

$$
\frac{d}{d \xi}\left(a^{2}+b^{2}\right)=0
$$

so that

$$
\frac{\delta \theta}{\theta}=-\frac{n a^{2} \phi^{2}}{2 \sigma \lambda}
$$

since

$$
\frac{d v}{d \xi}=\frac{\mathbf{I}}{\boldsymbol{\sigma}} .
$$

To get some estimate of the magnitude of this effect let us suppose that the cylinder is I centimetre in radius, and that $\phi$ is $\mathrm{I} / 40$. Since Young's modulus for ice is $6 \times 10^{10}$, $n$ is probably about $2.4 \times 10^{10}$, substituting these values we 
find

$$
\frac{\delta \theta}{\theta}=-\frac{\mathbf{I}}{400} \text { approximately, }
$$

so that $\delta \theta=-\cdot 68^{\circ} \mathrm{C}$.

So that in this case the ice on the surface would melt unless the temperature was lower than $-68^{\circ} \mathrm{C}$.

I30. Effect of surface tension on the freezing point. If a portion of a drop of water freezes, the formation of the ice will cause a diminution in the surface of separation of the water and air if the ice rises to the surface of the drop, to balance this however we have two fresh surfaces formed where the ice meets the water and air; the diminution in the first surface would tend to promote freezing, the formation of the other two would tend to prevent it, but as we do not know the surface tension between ice and water and between ice and air we cannot calculate which of these tendencies would have the upper hand.

I3 I. The effect of dissolved salt on the freezing point. When a salt solution freezes the salt appears to remain behind, and the ice from such a solution is identical with that from pure water. Thus when a portion of a salt solution freezes, the particles of salt are brought closer together, and work has therefore to be done upon them, the Lagrangian function therefore diminishes, and we see by equation (239) that the presence of the salt will tend to prevent the water from freezing. To calculate the magnitude of this effect, let $\zeta$ be the mass of the salt, then using the same notation as before, the mean Lagrangian function for the salt if the solution is dilute is

$$
\theta R \zeta \log \frac{v^{\prime} \rho_{0}}{\zeta}+\zeta f(\theta)-\zeta w_{3},
$$


where $w_{3}$ is the mean potential energy of unit mass of the salt. When the mass of ice is increased by $\delta \xi$ the only quantity which changes in the expression is $v^{\prime}$ which diminishes by $\delta \xi / \rho$.

Thus equation (239) becomes

$$
\frac{\delta \theta}{\theta}=-\frac{\mathrm{I}}{\lambda}\left\{\frac{\theta R \zeta}{\rho v^{\prime}}+\theta \delta\left(\frac{\Omega^{\prime}}{\rho}\right)+\delta f_{2}(\theta)\right\},
$$

where $\delta\left(\Omega^{\prime} / \rho\right)$ and $\delta f_{2}(\theta)$ are the changes in $\Omega^{\prime} / \rho$ and $f_{\mathbf{g}}(\theta)$ due to the salt. If $\varpi$ be the pressure due to the molecules of salt in the solution,

$$
\varpi=\frac{R \theta \zeta}{v^{\prime}},
$$

so that

$$
\frac{\delta \theta}{\theta}=-\frac{\mathbf{I}}{\lambda}\left\{\frac{\varpi}{\rho}+\theta \delta\left(\frac{\boldsymbol{\Omega}^{\prime}}{\rho}\right)+\delta f_{\mathrm{g}}(\theta)\right\} .
$$

If we suppose that the salt does not alter the properties of the solvent we have

$$
\frac{\delta \theta}{\theta}=-\frac{\varpi}{\lambda \rho} .
$$

Let us first suppose that the solvent is water; if we consider solutions whose strength is such that a number of grammes equal to the formula weight is dissolved in one litre of water, then $\varpi$ is about 22 atmospheres, or in absolute measure about $2 \cdot 2 \times 10^{7}, \lambda=80 \times 4^{\cdot} 2 \times 10^{7}, \theta=273$, and $\rho$ is unity; substituting these values we get

$$
\delta \theta=-\mathrm{r} \cdot 8^{\circ} \mathrm{C} .
$$

Raoult, Annales de Chimie et de Physique, v. III. p. 324, 1886 , found that solutions of this strength of many substances, chiefly organic salts, froze at $-1 \cdot 9^{\circ}$, but that the freezing points of solutions of salts and acids were generally lower than this; he attributed the increased effect to the dis- 
sociation of the molecules; it might however, as in the analogous cases we considered before, be due to the alteration of the properties of the solvent by the addition of the salt. It would also take place if there were any chemical action between the salt and solvent of such a nature that heat is evolved when the solution is diluted.

When the solvent is acetic acid, $\lambda=44^{\circ} 34 \times 4.2 \times 10^{7}$ (Landholt and Börnstein Tabellen) $\rho=1.05$ and $\theta=290$; substituting these values we get for the lowering of the freezing point of any solution of the same strength as before

$$
\delta \theta=-3.3^{\circ} \mathrm{C} .
$$

In this case Raoult found $\delta \theta=-3^{\circ} 9$.

When the solvent is benzine, $\lambda=29 \times 4^{\circ} 2 \times 10^{7}, \rho=\cdot 9$ and $\theta=275$, so that the lowering of the freezing point of $a$ solution of the same strength as before is

$$
\delta \theta=-54^{\circ} \mathrm{C} .
$$

Raoult found in this case that $\delta \theta$ was $-49^{\circ} \mathrm{C}$.

Raoult found that the effect of dissolved salts on the freezing points of acetic acid and benzine was much more regular than their effect on the freezing point of water. 


\section{CHAPTER XVII.}

THE CONNEXION BETWEEN ELECTROMOTIVE FORCE AND CHEMICAL CHANGE.

132. THE principle that when a system is in equilibrium the Hamiltonian function is stationary can be applied to determine the connexion between the electromotive force of a battery and the nature of the chemical combination which takes place when an electric current flows through it.

We shall begin by considering Grove's gas battery, as this is the case where the chemical changes seem on the whole to be the least complex. In this battery the two electrodes are covered with finely divided platinum, the upper half of one is surrounded by some gas, say hydrogen, while the lower half dips into acidulated water; the upper half of the other electrode is surrounded by some other gas, say oxygen, the lower half again dipping into acidulated water. The two electrodes are well coated with hydrogen and oxygen respectively. If the electrodes are connected a current will flow through the battery and the hydrogen and oxygen above the electrodes will gradually disappear, while the water will increase during the passage of the current.

To investigate the electromotive force of a battery of this kind let us suppose that the electrodes have got into a 
permanent condition, so that the gases attached to them are not altered during the passage of the current, let us also suppose that the electrodes are connected with the plates of a condenser whose capacity is $C$, these plates being made of the same material. Then if unit quantity of positive electricity flows from the plate of the condenser which is connected with the hydrogen electrode through the cell to the other plate, by Faraday's Law an electrochemical equivalent of hydrogen will appear at the electrode covered with oxygen and one of oxygen at the electrode covered with hydrogen; the hydrogen and the oxygen will combine and the result of the passage of the unit of electricity will be that an electrochemical equivalent of hydrogen and one of oxygen will disappear and an electrochemical equivalent of water will appear. The systems whose mean Lagrangian functions change during this process are (I) the condenser, (2) the hydrogen above one electrode, (3) the oxygen above the other, and (4) the water.

Let $Q$ be the quantity of positive electricity on the plate of the condenser connected with the oxygen electrode, and let $\xi, \eta, \zeta$ be the masses of the hydrogen and oxygen above the electrodes and of the acidulated water respectively.

The mean Lagrangian function for the condenser is

$$
-\frac{\mathrm{r}}{2} \frac{Q^{2}}{C} \text {. }
$$

The mean Lagrangian function for the hydrogen is $\xi L_{H}$ where using the same notation as hitherto,

$$
L_{H}=R_{1} \theta \log \frac{\rho_{0}}{\rho}+f_{1}(\theta)-w_{1} .
$$

The mean Lagrangian function for the oxygen is $\eta L_{O}$ where

$$
L_{o}=R_{2} \theta \log \frac{\rho_{0}^{\prime}}{\rho^{\prime}}+f_{\mathbf{s}}(\theta)-w_{2}
$$


and for the acidulated water $\zeta L_{w}$ where

$$
L_{w}=\gamma \theta+f_{3}(\theta)-w_{3} \text {. }
$$

Now when unit of electricity passes from the one plate to the other of the condenser, the electrochemical equivalent of hydrogen is carried to the oxygen and there combines with it at one electrode, while the electrochemical equivalent of oxygen is carried to and combines with the hydrogen at the other electrode. 'Thus if $\epsilon_{1}$ and $\epsilon_{2}$ are the electrochemical equivalents of hydrogen and oxygen, the net result of the process is that $Q$ has increased by unity, $\xi$ and $\eta$ diminished by $\epsilon_{1}$ and $\epsilon_{2}$ respectively, while $\zeta$ has increased by $\left(\epsilon_{1}+\epsilon_{2}\right)$. Hence by the principle that the Hamiltonian function is stationary when there is equilibrium we must have

$$
-\epsilon_{1} \frac{d}{d \xi}\left(\xi L_{H}\right)-\epsilon_{2} \frac{d}{d \eta}\left(\eta L_{O}\right)+\left(\epsilon_{1}+\epsilon_{2}\right) \frac{d}{d \zeta}\left(\zeta L_{w}\right)-\frac{Q}{C}=0 ;
$$

but $Q / C$ is the amount by which the potential of the plate connected to the oxygen electrode exceeds that of the one connected to the hydrogen electrode, in other words it is the electromotive force of the battery, which we shall call $p$, hence

$$
p=-\epsilon_{1} \frac{d}{d \xi}\left(\xi L_{H}\right)-\epsilon_{2} \frac{d}{d \eta}\left(\eta L_{O}\right)+\left(\epsilon_{1}+\epsilon_{2}\right) \frac{d}{d \zeta}\left(\zeta L_{w}\right) \ldots(240) .
$$

If $L_{w}$ be the mean Lagrangian function of unit mass of aqueous vapour above the acidulated water and in equilibrium with it, we have by $\S 83$

$$
\frac{d}{d \zeta}\left(\zeta L_{z v}\right)=\frac{d}{d \zeta^{\prime}}\left(\zeta^{\prime} L_{z v^{\prime}}\right)
$$

where $\zeta^{\prime}$ is the mass of the aqueous vapour, and

$$
L_{w^{\prime}}=R_{3} \theta \log \frac{\rho_{0}^{\prime \prime}}{\rho^{\prime \prime}}+f_{3}^{\prime}(\theta)-7{w^{\prime}}^{\prime} \text {. }
$$

Substituting these values in (240) we get 


$$
\begin{aligned}
& p=\epsilon_{1} R_{1} \theta \log \frac{\rho}{\rho_{0}}+\epsilon_{2} R_{2} \theta \log \frac{\rho^{\prime}}{\rho_{0}^{\prime}}-\left(\epsilon_{1}+\epsilon_{2}\right) R_{3} \theta \log \frac{\rho^{\prime \prime}}{\rho_{0}^{\prime \prime}} \\
& \begin{array}{r}
+\begin{array}{c}
\left.\epsilon_{1} R_{1}+\epsilon_{2} R_{2}-\left(\epsilon_{1}+\epsilon_{2}\right) R_{3}\right\} \theta-\epsilon_{1} f_{1}(\theta)-\epsilon_{2} f_{2}(\theta)+\left(\epsilon_{1}+\epsilon_{2}\right) f_{3}(\theta) \\
+\left\{\epsilon_{1} w_{1}+\epsilon_{2} w_{2}-\left(\epsilon_{2}+\epsilon_{2}\right) w_{3}^{\prime}\right\}
\end{array} \\
\text { But } \quad \epsilon_{1} R_{1}=2 \epsilon_{2} R_{2}=\left(\epsilon_{1}+\epsilon_{2}\right) R_{3} ;
\end{array}
\end{aligned}
$$

and by $\left(8_{3}\right)$

$$
-\epsilon_{1} f_{1}(\theta)-\epsilon_{2} f_{2}(\theta)+\left(\epsilon_{1}+\epsilon_{2}\right) f_{3}(\theta)
$$

is of the form

Lastly

$$
\begin{gathered}
A^{\prime} \theta+B \theta \log \theta . \\
\epsilon_{1} w_{1}^{\prime}+\epsilon_{2} w_{2}-\left(\epsilon_{1}+\epsilon_{2}\right) w_{3}^{\prime}
\end{gathered}
$$

is the loss of potential energy which occurs when an electrochemical equivalent of hydrogen combines with one of oxygen and may be measured by the quantity of heat developed by the combination of an electrochemical equivalent of hydrogen at the zero of absolute temperature; we shall denote it by $\epsilon_{1} q$, making these substitutions we see

$$
p=\epsilon_{1} R_{1} \theta \log \frac{\rho \rho^{\prime \frac{1}{2}}}{\rho^{\prime \prime}}+A \theta+B \theta \log \theta+\epsilon_{1} g \ldots \text { (24I), }
$$

where

$$
A=A^{\prime}+\epsilon_{1} R_{1} \log \frac{\rho_{0}^{\prime \prime}}{\rho_{0} \rho_{0}^{\prime 2}}
$$

hence we have

$$
\theta^{2} \frac{d^{2} p}{d \theta^{2}}-\theta \frac{d p}{d \theta}+p=\epsilon_{1} q
$$

Thus if we know the way in which $p$ depends upon $\theta$ we can determine $q$, so that by measurements of the electromotive force of a cell and the variations of this force with the temperature we can calculate the mechanical equivalent of the heat developed in the combination which takes place in the cell.

133. Equations (24x) and (242) are not confined to the case of the Gas Battery. We can prove in a similar way that if $p$ is the electromotive force of any battery where the solutions used are dilute, then

$$
p=\epsilon_{1} R, \theta \log \frac{\rho_{1}{ }^{a} \rho_{2}{ }^{b} \cdots}{\sigma_{1}{ }^{c} \sigma_{2}{ }^{d} \cdots}+A \theta+B \theta \log \theta+\epsilon q \ldots(243),
$$


where $\epsilon_{1}$ is the electrochemical equivalent of hydrogen, $R_{1}$ the value of $R$ for this gas, $\rho_{1}, \rho_{2} \ldots$ the masses in unit volume of those substances which disappear as the chemical action which produces the current goes on, while $\sigma_{1}, \sigma_{2} \ldots$ are the masses in unit volume of those which appear, $a, b, \ldots c, d, \ldots$ are the ratics of the electrochemical equivalents of the substances to that of hydrogen, divided by the molecular weight of the substance, $\epsilon q$ is the mechanical equivalent of the heat which would be evolved at the absolute zero of temperature by the chemical action which takes place when unit of electricity passes through the cell.

From this equation we get as before

$$
\theta^{2} \frac{d^{2} p}{d \theta^{2}}-\theta \frac{d p}{d \theta}+p=\epsilon q \ldots \ldots \ldots \ldots \ldots(244) .
$$

By v. Helmholtz's principle $\S(48) \theta d p / d \theta$ is the heat which must be supplied to the cell in order to keep the temperature constant when the unit of electricity passes through the cell, or in other words $-\theta d p \mid d \theta$ is the mechanical equivalent of the heat which is reversibly generated when unit of electricity passes through the cell. Now $p$ the work done in driving this quantity of electricity through the cell plus $-\theta d p / d \theta$ the heat reversibly generated must be equal to $\epsilon w$ the heat equivalent of the chemical action which takes place in the cell, hence by $(243)$ we have

$$
\epsilon q-\theta^{\mathrm{g}} \frac{d^{\mathrm{q}} p}{d \theta^{\mathrm{a}}}=\epsilon w \ldots \ldots \ldots \ldots(245) .
$$

Now $\epsilon ?$ and $\epsilon q$ are the mechanical equivalents of the heat developed by the same combination when it takes place at the temperatures $\theta$ and absolute zero respectively, and the difference between these quantities must be the difference between the mechanical equivalents of the quantities of heat required to raise them from zero to $\theta$ degrees in their 
combined and uncombined states. If we consider the case when two gases $A$ and $B$ combine to form two others $C$ and $D$, then if $c_{1}, c_{2}, c_{3}, c_{4}$ are the mechanical equivalents of the specific heats at constant volume of these gases, $\epsilon_{1}, \epsilon_{2}, \epsilon_{3}, \epsilon_{4}$ their electrochemical equivalents, if we start with $A$ and $B$ at zero and raise them to $\theta$ degrees and then let them combine, we shall spend $\left(\epsilon_{1} c_{1}+\epsilon_{2} c_{2}\right) \theta$ units of work in raising the temperature and gain $\epsilon w$ by their combination, so that the net result in our favour will be

$$
\epsilon w-\left(\epsilon_{1} c_{1}+\epsilon_{2} c_{2}\right) \theta \text {. }
$$

If we let them combine at zero temperature and then raised them to $\theta^{0}$ we should gain $\epsilon q$ and spend $\left(\epsilon_{3} c_{3}+\epsilon_{4} c_{4}\right) \theta$ units of work, hence since the balance of work in our favour must be the same in both cases, we have

$$
\epsilon q-\left(\epsilon_{3} C_{3}+\epsilon_{4} c_{1}\right) \theta=\epsilon \mho U-\left(\epsilon_{1} C_{1}+\epsilon_{2} C_{2}\right) \theta,
$$

and therefore by (245)

$$
\theta^{3} \frac{d^{2} p}{d \theta^{2}}=\left(\epsilon_{3} c_{3}+\epsilon_{4} c_{4}-\epsilon_{1} c_{1}-\epsilon_{2} c_{2}\right) \theta .
$$

But by (24I)

$$
\theta^{2} \frac{d^{2} p}{d \theta^{2}}=B \theta
$$

so that

$$
B=\epsilon_{3} c_{3}+\epsilon_{4} c_{4}-\epsilon_{1} c_{1}-\epsilon_{2} c_{2} \ldots \ldots \ldots \ldots(246) .
$$

If the combination is attended by the production of an amount of heat comparable with that which occurs when hydrogen and oxygen combine, then $\theta^{2} d^{2} p / d \theta^{2}$, which is comparable with the heat required to raise the temperature of the substances $\theta$ degrees and is therefore at the most a few hundred calories per gramme of substance, will be small compared with $q$, which is measured by thousands of calories, so that when the combination is attended by the evolution of a large quantity of heat we may at ordinary temperature neglect $\theta^{2} d^{2} p / d \theta^{2}$ and write 


$$
p-\theta \frac{d p}{d \theta}=\epsilon q \text {. }
$$

Since by Dulong's and Petit's law $c_{1}, c_{2}, c_{3}, c_{4}$ are inversely proportional to the combining weights of the gases $A, B, C, D$, we see that whenever the combination leaves the number of molecules unaltered $B$ will vanish and the equation

$$
p-\theta \frac{d p}{d \theta}=\epsilon q
$$

will be rigorously true. We see by this equation that when the electromotive force increases as the temperature increases the electromotive force is greater, while when the electromotive force diminishes as the temperature increases it is less than that calculated from the formula $p=\epsilon q$, which is often employed.

If $k$ be the coefficient of the chemical combination ( $\S$ I I 5). which goes on in the cell, i.e. the value of

$$
\frac{\rho_{1}^{a} \rho_{2}^{b}}{\sigma_{1}{ }^{c} \sigma_{2}^{d^{d}}}
$$

when the densities of the gases or solutions have the values they possess when in chemical equilibrium with each other, then since any small change cannot alter the value of the mean Lagrangian function of the gases or dilute solutions when in equilibrium, we get if we suppose the change is that which would take place if unit of electricity were to pass through the solutions

$$
\circ=\epsilon_{1} R_{1} \theta \log k+A \theta+B \theta \log \theta+\epsilon q \ldots \ldots(247) ;
$$

combining this with (245) we get

$$
p=\epsilon_{1} R_{1} \theta\left\{\log \frac{\rho_{1}^{a} \rho_{2}^{b} \cdots}{\sigma_{1}^{c} \sigma_{2}{ }^{d} \cdots}-\log k\right\}
$$

or

$$
\log k=\log \frac{\rho_{1}^{a} \rho_{9}^{b}}{\sigma_{1}{ }^{c} \sigma_{2}{ }^{d}}-\frac{p}{\epsilon_{1} R_{1} \theta} \ldots \ldots \ldots \ldots\left(24^{8}\right) \text {. }
$$


This equation affords a very easy method of finding the coefficient of any chemical combination if we can make a cell in which this combination takes place, for then if we measure the electromotive force and the densities of the solutions, equation (247) will at once give $k$. Thus the Daniell's cell enables us to calculate the coefficient of the combination

$$
\mathrm{Zn}+\mathrm{H}_{2} \mathrm{SO}_{4}+\mathrm{CuSO}_{4}=\mathrm{ZnSO}_{4}+\mathrm{H}_{2} \mathrm{SO}_{4}+\mathrm{Cu} \text {. }
$$

Here if $\rho$ and $\sigma$ are the masses per unit volume of the $\mathrm{CuSO}_{4}$ and $\mathrm{ZnSO}_{4}$ respectively when there is chemical equilibrium

$$
\log k=\log \frac{\rho^{\frac{1}{2}}}{\sigma^{\frac{1}{2}}},
$$

so that if $\rho^{\prime}$ and $\sigma^{\prime}$ are the densities of the $\mathrm{CuSO}_{4}$ and the $\mathrm{ZnSO}_{4}$ when the electromotive force is $p$ we have

$$
\log k=\frac{\mathrm{x}}{2} \log \frac{\rho^{\prime}}{\sigma^{\prime}}-\frac{p}{\epsilon_{1} R_{1} \theta} .
$$

Now at $\circ^{\circ} \mathrm{C} . \epsilon_{1} R R_{1} \theta$ is nearly $10^{6}$ and $p$ is about $10^{8}$ so that

$$
\log k=\frac{\mathrm{r}}{2} \log \frac{\rho^{\prime}}{\sigma^{\prime}}-100,
$$

or approximately since for ordinary strengths of solution $\log \rho^{\prime} / \sigma^{\prime}$ is small compared with roo

$$
\log _{\varepsilon} \frac{\rho}{\sigma}=-200,
$$

hence we see that in this case when there is equilibrium practically all the sulphuric acid goes to the zinc.

If we determined the electromotive force of a battery when lead wire dipped respectively into acid solutions of lead nitrate and lead chloride, we should be able by equation (247) to determine the coefficient of the action

$$
2 \mathrm{HCl}+\mathrm{Pb}\left(\mathrm{NO}_{3}\right)_{2}=2 \mathrm{HNO}_{3}+\mathrm{PbCl}_{2} \text {, }
$$


and so determine the way in which lead divides itself between hydrochloric and nitric acids.

If we return now to the hydrogen and oxygen gas battery, equation (247) is for this case

$$
p=\epsilon_{1} R_{1} \theta \log \frac{\rho \rho^{\prime \frac{1}{2}}}{\rho^{\prime \prime} k} \ldots \ldots \ldots \ldots \ldots . . . . .(249) \text {. }
$$

We can easily deduce from this equation the way in which the electromotive force of a gas battery depends upon the pressure of the gases in the vessels above the electrodes. If $p_{1}$ is the electromotive force of the battery when the densities of the hydrogen and oxygen are $\rho, \rho^{\prime}$ respectively, $p_{2}$ the electromotive force when the densities are $\sigma$ and $\sigma^{\prime}$, then we have by (249)

$$
p_{2}-p_{1}=\epsilon_{1} R_{1} \theta \log \frac{\sigma \sigma^{\prime \frac{1}{2}}}{\rho \rho^{\frac{1}{2}}} \text {. }
$$

If the densities of the oxygen and hydrogen were diminished one thousand times then at the temperature $\circ$ C. since $\epsilon_{1}=10^{-4}, R_{1} \theta=\mathrm{I} \cdot \mathrm{I} \times 10^{10}$

$$
\begin{aligned}
p_{2}-p_{1} & =-I \cdot 1 \times 10^{6} \log _{c} 10^{\frac{9}{2}} \\
& =-1 \cdot 1 \times 4.5 \times 2 \cdot 3 \times 10^{6} \\
& =-1 \cdot 14 \times 10^{7} \text { approximately, }
\end{aligned}
$$

so that the electromotive force is diminished by rather less than the ninth of a volt. By making the densities of the gases above the electrodes sufficiently small we could reverse the electromotive force, though in the case when the gases are oxygen and hydrogen the rarefaction required would be more than could practically be obtained.

The diminution in the electromotive force caused by rarefaction does not however depend upon the magnitude of the electromotive force of the battery, so that in the case

T. D. 
of gas batteries with small electromotive forces this reversal might be practicable.

The condensation which accompanies the combination of oxygen and hydrogen diminishes the effect of rarefaction; if the combination were to take place without condensation, the diminution in the electromotive force caused by diminishing the density one thousand times would be about one-seventh of a volt.

We see too from equation (249) that the electromotive force in all cases tends to produce a current the chemical action of which would make the densities of the gases or dilute solutions approach the values they have when in chemical equilibrium with each other. When they have these values the electromotive force of the battery is zero, and the electromotive force is in one direction or the opposite according as there is more or less of some substance present than there would be if the mixture of gases or dilute solutions were in chemical equilibrium.

Experiments on the electromotive force of gas batteries charged with various gases have been made by Pierce (Wiedemann's Annalen, vir. p. 98). The following table taken from his paper gives the electromotive force of a large number of batteries at $15^{\circ} \mathrm{C}$. and of a few at $75^{\circ}-80^{\circ} \mathrm{C}$.

It will be seen from this table that the effect of an increase in temperature on the electromotive force of gas batteries is very variable, for of the five batteries whose electromotive forces were determined at different temperatures, the electromotive forces of three were less and of two greater at the high temperature than the low. 
ELECTROMOTIVE FORCE AT $18^{\circ} \mathrm{C}$.

\begin{tabular}{|c|c|c|c|c|c|}
\hline Gases. & $\begin{array}{l}\text { Fluid between the } \\
\text { electrodes. }\end{array}$ & $\begin{array}{l}\text { Ratio of elec- } \\
\text { tromotive } \\
\text { force to that } \\
\text { of a Daniell. }\end{array}$ & Gases. & Fluid. & $\begin{array}{l}\text { Electro- } \\
\text { motive } \\
\text { force. }\end{array}$ \\
\hline $\begin{array}{l}\mathrm{H} \text { and } \mathrm{O} \\
\mathrm{H} \text { and } \mathrm{N}_{2} \mathrm{O} \\
\mathrm{H} \text { and } \mathrm{CO}_{2} \\
\mathrm{H} \text { and } \mathrm{NO}^{2} \\
\mathrm{H} \text { and air } \\
\mathrm{H} \text { and } \mathrm{H}_{2} \mathrm{O} \\
\mathrm{H} \text { and } \mathrm{CO} \\
\mathrm{H} \text { and } \mathrm{O} \\
\mathrm{H} \text { and } \mathrm{CO} \\
\mathrm{H} \text { and } \mathrm{NO} \\
\mathrm{H} \text { and } \mathrm{O} \\
\mathrm{H} \text { and } \mathrm{O} \\
\mathrm{H} \text { and } \mathrm{O} \\
\mathrm{H} \text { and } \mathrm{CO}_{2} \\
\mathrm{H} \text { and } \mathrm{NO}^{2}\end{array}$ & $\begin{array}{c}\text { water } \\
\text { water } \\
\text { water } \\
\text { water } \\
\text { water } \\
\text { water } \\
\text { water } \\
\mathrm{H}_{2} \mathrm{SO}_{4}+\text { water } \\
\mathrm{H}_{2} \mathrm{SO}_{4}+\text { water } \\
\mathrm{H}_{2} \mathrm{SO}_{4}+\text { water } \\
\mathrm{Na}_{2} \mathrm{SO}_{4}+\text { water } \\
\mathrm{K}_{2} \mathrm{SO}_{4}+\text { water } \\
\mathrm{ZnSO}_{4}+\text { water } \\
\mathrm{ZnSO}_{4}+\text { water } \\
\mathrm{ZnSO}_{4}+\text { water }\end{array}$ & $\begin{array}{l}\cdot 874 \\
\cdot 790 \\
.981 \\
.933 \\
.807 \\
.807 \\
.404 \\
.926 \\
.892 \\
.768 \\
.698 \\
.698 \\
.771 \\
.820 \\
.860\end{array}$ & $\begin{array}{l}\mathrm{I} \text { and } \mathrm{Br} \\
\mathrm{H} \text { and } \mathrm{Br} \\
\mathrm{H} \text { and } \mathrm{Br} \\
\mathrm{O} \text { and } \mathrm{Br} \\
\mathrm{O} \text { and } \mathrm{I} \\
\mathrm{H} \text { and } \mathrm{I} \\
\mathrm{H} \text { and } \mathrm{NO} \\
\mathrm{H} \text { and } \mathrm{O} \\
\mathrm{H} \text { and } \mathrm{Cl} \\
\mathrm{H} \text { and } \mathrm{Cl} \\
\mathrm{H} \text { and } \mathrm{Cl} \\
\mathrm{H} \text { and } \mathrm{O} \\
\mathrm{H} \text { and } \mathrm{CO} \\
\mathrm{H} \text { and } \mathrm{NO}^{2}\end{array}$ & $\begin{array}{l}\quad \text { water } \\
\mathrm{NaBr} \text { + water } \\
\mathrm{KBr} \text { + water } \\
\mathrm{KBr} \text { + water } \\
\mathrm{KI} \text { + water } \\
\mathrm{KI} \text { + water } \\
\mathrm{HCl} \text { + water } \\
\mathrm{HCl} \text { + water } \\
\mathrm{HCl} \text { + water } \\
\mathrm{KCl} \text { + water } \\
\mathrm{NaCl} \text { + water } \\
\mathrm{NaCl} \text { + water } \\
\mathrm{NaCl} \text { + water } \\
\mathrm{NaCl} \text { + water }\end{array}$ & $\begin{array}{c}\cdot 335 \\
1 \cdot 252 \\
1 \cdot 253 \\
\cdot 5 \\
\cdot 057 \\
.861 \\
\cdot 765 \\
\cdot 855 \\
1 \cdot 36 \\
1 \cdot 39 \\
1 \cdot 39 \\
\cdot 766 \\
\cdot 846 \\
\cdot 750\end{array}$ \\
\hline
\end{tabular}

ELECTROMOTIVE FORCE AT $75^{\circ} \mathrm{C}-80^{\circ} \mathrm{C}$.

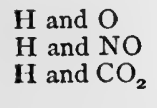

\begin{tabular}{l|l} 
water & $\cdot 828$ \\
water & $\cdot 945$ \\
water & $\cdot 875$
\end{tabular}

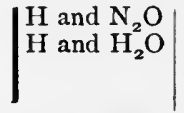

water water

The electromotive force of the hydrogen and oxygen gas battery where $\epsilon q=3.4 \times 4^{.2} \times \mathrm{IO}^{7}$ is less than that given by the formula (246) even when the variation of the electromotive force with temperature is taken into account. This seems most probably to arise from the arrangements being such that the complete combination of the hydrogen and oxygen contemplated in the preceding theory would not take place, for we see from the table that the substitution of acidulated water for water between the terminals increases the electromotive force, this change favours the production of ozone instead of oxygen when the current passes and so increases the chance of complete combination. 
In the case of the hydrogen and chlorine battery with a solution of hydrochloric acid between the electrodes, $q$ in formula (244) will be the heat in mechanical units given out in the combination of one gramme of hydrogen with chlorine plus the heat given out when 36.5 grammes of hydrochloric acid are dissolved in a large quantity of water.

The gas battery will work even if we have the same gas (say hydrogen) above the electrodes provided it is at different pressures. In this case on closing the circuit there will be no change in the volume of the liquid between the terminals, but when the unit of electricity passes through the battery an electrochemical equivalent of hydrogen will be transferred from the vessel where the pressure is high to the one where it is low. The electromotive force in this case is easily seen to be

$$
\epsilon_{1} R_{1} \theta \log \rho / \sigma,
$$

where $\rho$ and $\sigma$ are the densities of the hydrogen (or oxygen) in the two vessels, at $0^{\circ} \mathrm{C}$. this equals

$$
{ }_{10}{ }^{6} \log \rho / \sigma \text { approximately, }
$$

so that if the density in one vessel is $e$ times that in the other the electromotive force will be one-hundredth of a volt.

In fact when we have any arrangement in which the passage of an electric current in a certain direction increases the Lagrangian function of the system, there will be an electromotive force tending to produce a current in this direction and equal to the increase in the mean Lagrangian function produced by the passage of unit of electricity.

134. We can sometimes transform equation (244) by means of the following considerations. If we have a mixture of chemical reagents in various proportions we can in many cases though not in all find a temperature at which they 
would be in equilibrium if mixed in these proportions. Let us suppose that it is possible to find a temperature $\theta_{0}$ at which the reagents constituting the battery would be in equilibrium in the proportion in which they exist in the battery at the temperature $\theta$.

Then iy (243)

and by (246)

$$
\frac{p}{\bar{\theta}}=\epsilon_{1} R_{1} \log \frac{\rho_{1}^{a} \rho_{2}{ }^{b} \cdots}{\sigma_{1}^{c} \sigma_{9}{ }^{d} \cdots}+A+B \log \theta+\frac{\epsilon q}{\theta},
$$

$$
\mathrm{o}=\epsilon_{1} R_{1} \log \frac{\rho_{1}^{a} \rho_{\mathrm{g}}^{b} \cdots}{\sigma_{1}{ }^{c} \sigma_{\mathrm{g}}{ }^{d} \cdots}+A+B \log \theta_{0}+\frac{\epsilon q}{\theta_{0}} .
$$

Subtracting these equations we get

$$
\frac{p}{\theta}=\epsilon q\left(\frac{\mathrm{r}}{\theta}-\frac{\mathrm{r}}{\theta_{0}}\right)+B \log \frac{\theta}{\theta_{0}} \ldots \ldots \ldots \ldots . .(250) .
$$

If a considerable quantity of heat is given out by the combination which takes place when unit of electricity passes through the cell, then at ordinary temperatures the last term on the right-hand side of this equation will be small compared with the first and we may write equation $\left(25^{\circ}\right)$ in the form

$$
p=\epsilon q\left(\mathbf{I}-\frac{\theta}{\theta_{0}}\right) .
$$

An equation identical in form with this is given by Professor Willard Gibbs in a letter to the Electrolysis Committee of the British Association (British Association Report, I886, p. 388). According to Prof. Gibbs $\theta_{0}$ is the highest temperature at which the radicles can combine with evolution of heat, while according to our view it is the temperature at which the chemical system forming the battery would be in equilibrium, and as it is not always possible to find such a temperature the formula is not of universal application. We see that for all cells to which the formula 
can be applied the temperature coefficient of the electromotive force must be negative, and therefore by v. Helmholtz's principle, the passage of the current through the cell must be attended by the evolution of heat. When the temperature $\theta_{0}$ exists it is given by the equation

$$
\theta_{0}=-\frac{\epsilon q}{\frac{d p}{d \theta}} \text { approximately. }
$$

We shall now investigate under what conditions it is possible to find a temperature $\theta_{0}$ at which the system would be in equilibrium. We shall consider the case of the equilibrium of four substances $(A),(B),(C),(D)$.

If $\rho_{1}, \rho_{2}, \sigma_{1}, \sigma_{2}$ are the densities of $(A),(B),(C),(D)$ when there is equilibrium we have by equation $(246)$

$$
\mathrm{o}=\epsilon_{1} R_{1} \theta \log \frac{\rho_{1}^{a} \rho_{\mathrm{g}}^{b}}{\sigma_{1}^{c}{ }^{c} \sigma_{\mathrm{g}}}+A \theta+B \theta \log \theta+\epsilon q .
$$

Now if $e_{1}, e_{2}, e_{3}, e_{4}$ are the electrochemical equivalents and $c_{1}, c_{3}, c_{3}, c_{4}$ the specific heats at constant volume of the substances $(A),(B),(C),(D)$ respectively, then by equation (246)

so that

$$
B=e_{4} c_{4}+e_{3} c_{3}-e_{2} c_{2}-e_{1} c_{1},
$$

$$
\frac{\rho_{1}^{a} \rho_{2}^{b}}{\sigma_{1}^{c} \sigma_{2}^{d}}=C \theta^{\frac{\left(e_{1} c_{1}+e_{2} c_{2}-e_{2} c_{3}-e_{4} c_{4}\right)}{R_{1} e_{1}}} e^{-\frac{q e}{R_{1} \theta e_{1}}} .
$$

Now if $M_{1}, M_{2}, M_{3}, M_{4}$ are the molecular weights of the substances

$$
\begin{array}{ll}
\frac{e_{1}}{\epsilon_{1}}=M_{1} a, & \frac{e_{3}}{\epsilon_{1}}=M_{3} c, \\
\frac{e_{g}}{\epsilon_{1}}=M_{2} b, & \frac{e_{4}}{\epsilon_{1}}=M_{4} d,
\end{array}
$$


and for gases by Dulong and Petit's Law

$$
\begin{gathered}
M_{1} c_{1}=M_{2} c_{2}=M_{3} c_{3}=M_{4} c_{4}=\text { c say } \\
\frac{\rho_{1}^{a} \rho_{3}^{b}}{\sigma_{1}^{c} \sigma_{2}^{d}}=C \theta^{\frac{(a+b-c-d) \varepsilon}{R_{2}}} e^{-\frac{g e}{R_{1} \theta_{1}}}
\end{gathered}
$$

$\epsilon q$ is the heat given out when the quantity of $(A)$ decomposed by one unit of electricity combines with the equivalent quantity of $B$. Let us first suppose that this quantity is positive, and consider the following cases.

Ist case. When $a+b=c+d$, i.e. when there is no change in volume on combination. In this case as $\theta$ increases from zero to infinity $\rho_{1}{ }^{a} \rho_{2}{ }^{b} / \sigma_{1}{ }^{c} \sigma_{2}{ }^{d}$ ranges from zero to $C$, and therefore since it never exceeds $C$ it is not always possible to find a temperature which should be one of equilibrium for any arbitrarily chosen set of values of $\rho_{1}, \rho_{2}, \sigma_{1}, \sigma_{2}$.

and case. When $a+b<c+d$, i.e. when there is an increase in volume after combination. In this case as $\theta$ increases from zero to infinity $\rho_{1}{ }^{a} \rho_{2}{ }^{b} / \sigma_{1}{ }^{c} \sigma_{2}{ }^{d}$ starts from zero then reaches a maximum and decreases again to zero, so that again as $\rho_{1}{ }^{a} \rho_{2}{ }^{b} / \sigma_{1}{ }^{c} \sigma_{2}{ }^{d}$ never exceeds a certain maximum it is not always possible to find a temperature which should be one of equilibrium for any arbitrarily chosen set of values of $\rho_{1}, \rho_{8}, \sigma_{1}, \sigma_{z}$.

$3^{\text {rd }}$ case. When $a+b>c+d$, i.e. when there is a diminution in volume after combination. In this case as $\theta$ increases from zero to infinity $\rho_{1}{ }^{a} \rho_{8}{ }^{b} / \sigma_{1}{ }^{c} \sigma_{2}{ }^{d}$ also increases from zero to infinity, so that in this case it is always possible to find a temperature which should be one of equilibrium for any arbitrarily chosen set of values of $\rho_{1}, \rho_{2}, \sigma_{1}, \sigma_{2}$.

We see too that when the combination is attended with an absorption of heat it is in general only possible to find a temperature which shall be one of equilibrium for any arbitrarily 
chosen set of values of $\rho_{1}, \rho_{2}, \sigma_{1}, \sigma_{2}$ when the combination is attended by an increase of volume.

Summing up the results of this investigation we see that equation (250) can only in general be applied to cases where the reaction producing heat is accompanied by a diminution in volume.

In these cases where $\rho_{1}{ }^{a} \rho_{\mathbf{g}}{ }^{b} / \sigma_{1}{ }^{c} \sigma_{g}^{d}$ has a maximum value at a finite temperature the mixture of gases after passing this temperature will be in an unstable state, for any increase in the temperature will promote combination and produce an evolution of heat which will increase the temperature still further. 


\section{CHAPTER XVIII.}

IRREVERSIBLE EFFECTS.

135. WE have hitherto left out of consideration the effect of such things as frictional and electrical resistances which destroy the reversibility of any process in which they play a part. If however we take the view that the properties of matter in motion, as considered in abstract dynamics, are sufficient to account for any physical phenomenon, then irreversible processes must be capable of being explained as the effect of changes all of which are reversible.

It would not be sufficient to explain these irreversible effects by means of ordinary dynamical systems involving friction, as friction itself ought, on this view, to be explained by means of the action of frictionless systems.

But if every physical phenomenon can be explained by means of frictionless dynamical systems each of which is reversible, then it follows that if we could only control the phenomenon in all its details, it would be reversible, so that as was pointed out by Maxwell, the irreversibility of any system is due to the limitation of our powers of manipulation. The reason we can not reverse every process is because we only possess the power of dealing with the molecules en masse and not individually, while the reversal of some processes 
would require the reversal of the motion of each individual molecule.

We are not only unable to manipulate very minute portions of matter, but we are also unable to separate events which follow one another with great rapidity. The finite time our sensations last causes any phenomenon which consists of events following each other in rapid succession to present a blurred appearance, so that what we perceive at any moment is not what is happening at that moment, but merely an average effect which may be quite unlike the actual effect at any particular instant. In consequence of the finiteness of the time taken by our senses to act, we are incapable of separating two events which happen within a very short interval of each other, just as the finiteness of the wave length of light prevents us from seeing any separation between two points which are very close together. Thus if we observe any effect we cannot tell by our senses whether it represents a steady state of things or a state which is rapidly changing, and whose mean is what we actually observe. We are therefore at liberty, if it is more convenient for the purposes of explanation, to look upon any effect as the average of a series of rapidly changing effects of a different kind.

Let us now consider the case of a system whose motion is such that in order to represent it frictional terms proportional to the velocity have to be introduced, and let us assume at first that the motion is represented at each instant by the equations with these terms in, so that the dynamical equations are not equations which are merely true on the average.

It might appear at first sight as if we could explain the frictional terms in the equations of motion as arising from the connexion of subsidiary systems with the original system 
just as in $\S$ II we explained the "positional" forces as due to changes in the motion of a system connected with the original system. Let us suppose for a moment that this is possible. Then if $T$ is the kinetic energy of the original system, and $T^{\prime}$ that of the subsidiary system whose motion is to explain the frictional forces, we have, by Lagrange's equations,

$\frac{d}{d t} \frac{d T}{d \dot{x}}-\frac{d T}{d x}-\frac{d}{d t} \frac{d T^{\prime}}{d \dot{x}}-\frac{d T^{\prime}}{d x}+\frac{d V}{d x}=$ external force of type $x$;

thus the term

$$
-\frac{d}{d t} \frac{d T^{\prime}}{d \dot{x}}-\frac{d T^{\prime}}{d x}
$$

must be equal to the "frictional term" which is proportional to $\dot{x}$. For this to be the case, it is evident that $T^{\prime}$ must involve $\dot{x}$. The momentum of the system is, however, $d\left(T+T^{\prime}\right) / d \dot{x}$, and this momentum must be the same as that given by the ordinary expression in Rigid Dynamics, viz. $d T / d \dot{x}$. If these two expressions are identical, $d T^{\prime} / d \dot{x}$ must vanish for all values of $\dot{x}$, that is, $T^{\prime}$ cannot involve $\dot{x}$, which is inconsistent with the condition necessary in order that the motion of the subsidiary system should give rise to the "frictional" terms. Hence we conclude that the frictional terms cannot be explained by supposing that any subsidiary system with a finite number of degrees of freedom is in connexion with the original system.

If we investigate the case of a vibrating piston in connexion with an unlimited volume of air, we shall find that the waves starting from the piston dissipate its energy just as if it were resisted by a frictional force proportional to its velocity; this, however, is only the case when the medium surrounding the piston is unlimited, when it is bounded by fixed obstacles the waves originated by the piston get 
reflected from the boundary, and thus the energy which went from the piston to the air gets back again from the air to the piston. Thus the frictional terms cannot be explained by the dissipation of the energy by waves starting from the system and propagated through a medium surrounding it, for in this case it would be possible for energy to flow from the subsidiary into the original system, while, if the frictional terms are to be explained by a subsidiary system in connexion with the original one, the connexion must be such that energy can flow from the original into the subsidiary system, but not from the subsidiary into the original system.

Hence we conclude that the equations of motion, when they contain frictional terms, represent the average motion of the system, but not the motion at any particular instant.

Thus, to take an example, let us suppose that we have a body moving rapidly through a gas; then, since the body loses by its impacts with the molecules of a gas more momentum than it gains from them, it will be constantly losing momentum, and this might on the average be represented by the introduction of a term expressing a resistance varying as some power of the velocity; but the equations of motion, with this term in, would not be true at any instant, neither when the body was striking against a molecule of the gas, nor when it was moving freely and not in collision with any of the molecules. Again, if we take the resistance to motion in a gas which arises from its own viscosity, the kinetic theory of gases shows that the equations of motion of the gas, with a term included expressing a resistance proportional to the velocity, are not true at any particular instant, but only when the average is taken over a time which is large compared with the time a molecule takes to traverse its own free path. 
Since frictional forces cannot be explained by means of a system in a uniform state, we shall consider the dynamics of a system which is subject to the action of forces which last only for a short time but which recur very frequently.

Let us suppose that in the expression for the Lagrangian function of the system we are considering there is a term $L^{\prime}$ which is intermittent. It has for some small time a finite value, then vanishes, then springs into existence again, then vanishes, and so on, repeating its value $n$ times in a second. We shall for brevity speak of each of the epochs during which the function $L^{\prime}$ has a finite value as a collision, and shall call $n$ the number of collisions per second. For example, in the case of a body moving through a gas $L^{\prime}$ may be the part of the Lagrangian function which represents the action of a molecule of the gas on the body, when the body is in collision with a molecule $L^{\prime}$ has a finite value, when however the body is free from collision $L^{\prime}$ is so small that it may be assumed to be zero without appreciable error.

Lagrange's equation corresponding to the coordinate $x$ is, if $L$ is the steady part of the Lagrangian function,

$$
\frac{d}{d t} \frac{d L}{d \dot{x}}-\frac{d L}{d x}+\frac{d}{d t} \frac{d L^{\prime}}{d \dot{x}}-\frac{d L^{\prime}}{d x}=0 .
$$

Integrating this equation over a time $T$ we get

$$
\int_{0}^{T}\left(\frac{d}{d t} \frac{d L}{d \dot{x}}-\frac{d L}{d x}\right) d t+\left[\frac{d L^{\prime}}{d \dot{x}}\right]_{0}^{T}-\int_{0}^{T} \frac{d L^{\prime}}{d x}=0 .
$$

Now unless the structure of the system is steadily chang. ing $\left[d L^{\prime} / d \dot{x}\right]_{0}^{T}$ will either vanish or be exceedingly small, so that in general we may neglect it and write

$$
\int_{0}^{T}\left(\frac{d}{d t} \frac{d L}{d \dot{x}}-\frac{d L}{d x}\right) d t-\int_{0}^{T} \frac{d L^{\prime}}{d x} d t=0 \ldots \ldots(25 \mathrm{I})
$$


Let us choose $T$ so that though a great many collisions occur in this time, yet the values of $x, \dot{x}, \ddot{x}$ are not changed in it by a finite amount.

Now if $\tau$ be the time a collision lasts and if there are $n$ of them per second

$$
\int_{0}^{T} \frac{d L^{\prime}}{d x} d t=n T \int_{0}^{\tau} \frac{d L^{\prime}}{d x} d t \ldots \ldots \ldots \ldots(252) ;
$$

if as in a numerous class of cases $x$ may be supposed to remain constant during the collision, we may write $\left(25^{2}\right)$ as

$$
\int_{0}^{T} \frac{d L^{\prime}}{d x} d t=n T \frac{d \chi}{d x}
$$

where

$$
\chi=\int_{0}^{\tau} L^{\prime} d t
$$

Since

$$
\int_{0}^{T}\left(\frac{d}{d t} \frac{d L}{d \dot{x}}-\frac{d L}{d x}\right) d t=T\left(\frac{d}{d t} \frac{d L}{d \dot{x}}-\frac{d L}{d x}\right)
$$

equation (25 I) becomes

$$
\frac{d}{d t} \frac{d L}{d \dot{x}}-\frac{d L}{d x}=n \frac{d x}{d x} .
$$

Thus the effect produced by these intermittent forces is the same as that which would be produced by a steady force $X$ of type $x$ and given by the equation

$$
X=n \frac{d x}{d x} \text {. }
$$

Similarly they would produce the same effect as a force $Y$ of type $y$ where

$$
Y=n \frac{d \chi}{d y} .
$$

If $d \chi / d x, d \chi / d y$ do not involve the velocities $\dot{x}, \dot{y}$ and if $n$ the number of collisions per second is a linear function 
of these velocities, these forces will be of the character of frictional forces.

If $n$ does not involve the coordinates $x, y$ explicitly then we have

$$
\frac{d Y}{d x}=\frac{d X}{d y} \ldots \ldots \ldots \ldots \ldots \ldots(253),
$$

the consequences of this equation will be similar to those developed in $\$ 44$. Thus for example suppose we were to find that the logarithmic decrement of the torsional vibrations of a wire depended on the extension of the wire, then it would follow from $(252)$ that when the wire was vibrating there would be a force tending to alter its length. If the frictional resistance to the torsional vibrations were $\mu \dot{\theta}$, where $\dot{\theta}$ is the angular velocity of a pointer attached to the wire, then if the above equation is true, there would be a force $X$ tending to lengthen the wire and given by the equation

$$
\frac{d X}{d \theta}=-\dot{\theta} \frac{d \mu}{d x}, \text { where } x \text { is the length of the wire. }
$$

Thus if $\frac{d \mu}{d x}$ is constant we have

$$
X=-\theta \dot{\theta} \frac{d \mu}{d x} .
$$

Whence it follows that if the torsional vibrations were periodic there would be a force tending to produce longitudinal vibrations of half their period; or again, if the viscosity of an iron wire were altered by magnetization there would be a periodic magnetizing force acting on a vibrating wire whose period would be half that of the torsional vibrations.

The relation (253) is only satisfied when $n$ is independent of $x$ and $y$, if $n$ is a function of these quantities we shall have the relation 


$$
\frac{d Y}{d x}-\frac{d X}{d y}=X \frac{d \log n}{d y}-Y \frac{d \log n}{d x}
$$

instead, and if we consider forces of a third type $z$, the two additional relations

and

$$
\begin{aligned}
& \frac{d X}{d z}-\frac{d Z}{d x}=Z \frac{d \log n}{d x}-X \frac{d \log n}{d z} \\
& \frac{d Z}{d y}-\frac{d Y}{d z}=Y \frac{d \log n}{d z}-Z \frac{d \log n}{d y}
\end{aligned}
$$

so that

$$
Z\left(\frac{d Y}{d x}-\frac{d X}{d y}\right)+Y\left(\frac{d X}{d z}-\frac{d Z}{d x}\right)+X\left(\frac{d Z}{d y}-\frac{d Y}{d z}\right)=0 \ldots(254)
$$

In these relations $X, Y, Z$ are only those parts of the forces of types $x, y, z$ which are intermittent in their action.

If from the nature of the case we can see that the number of collisions is independent of some one coordinate $x$, then it follows from the above equations that

$$
\log n=\int \frac{\mathrm{I}}{X}\left(\frac{d Y}{d x}-\frac{d X}{d y}\right) d y+\int \frac{\mathrm{I}}{X}\left(\frac{d Z}{d x}-\frac{d X}{d z}\right) d z .
$$

If the viscous forces arise from collisions with several distinct systems, instead of with one as we have hitherto assumed, we shall have

$$
\begin{aligned}
& X=n_{1} \frac{d \chi_{1}}{d x}+n_{2} \frac{d \chi_{2}}{d x}+\ldots \\
& Y=n_{1} \frac{d \chi_{1}}{d y}+n_{2} \frac{d \chi_{2}}{d y}+\ldots
\end{aligned}
$$

where $n_{1}, n_{2}$ are the numbers of collisions per second with the systems (1), (2)... respectively, and

$$
\dot{\chi}_{r}=\int_{0}^{r} L_{r}^{\prime} d t
$$

where $L_{r}^{\prime}$ is the Lagrangian function of the $r$ th system. 
If $n_{1}, n_{\mathrm{g}} \ldots$ are independent of $x, y$ then as before

$$
\frac{d X}{d y}=\frac{d Y}{d x},
$$

but if $n_{1}, n_{2}$ involve the coordinates $x, y$, then the relation (253) must be replaced by one involving higher differential coefficients.

The preceding considerations show that in those cases where the viscous forces are due to "collisions" we have several criteria the fulfilment or non-fulfilment of which will afford us information about the constitution of the system. Thus if $(\mathbf{2 5 2})$ is not fulfilled we conclude that the number of collisions depends upon the value of the coordinates, if (253) is not fulfilled we conclude that the viscous forces are due to collisions with more systems than one and so on.

There is a great dearth of experiments on the influence of various physical conditions on viscous forces except when these forces are those which resist the passage of electricity through conductors. It does not seem probable however that in this case the resistance can be due to a succession of impulses whose number is proportional to the strength of the current; for the case is not analogous to that of a viscous force depending on the change of shape or configuration of a system, where we might reasonably expect the number of effective collisions to be proportional to the velocity of the change.

In order to get some idea as to how discontinuous forces can produce the effect of electric resistance, let us consider some cases in which effects analogous to resistance are produced by a succession of changes following one another in quick succession. A very good example of a case of this kind is the arrangement given by Maxwell (Electricity and Magnetism, II. p. 385) for measuring in

T. D. 
electromagnetic measure the capacity of a condenser, in which by means of a tuning fork interruptor the plates of a condenser are alternately connected with the poles of a battery and with each other. If the rate of discharge is very rapid, this arrangement of condenser and tuning fork produces the same effect as a resistance $\mathrm{I} / n C$ where $C$ is the capacity of the condenser and $n$ the number of times it is discharged per second. Thus in this case a combination of induction and discharge produces the same effect as a resistance. Another case in which the conditions are plainly discontinuous but which produces the same effect as a continuous current, if the rate of alternation is sufficiently rapid, is when electricity passes through a closed glass tube filled with air. If electrodes are fused into the tube and connected to an electrical machine in action there will be no discharge of electricity across the tube until the electromotive force gets large enough to break down the electric strength of the air, when a spark will pass, an interval will elapse before the second spark passes, during which the electromotive force inside the tube will be increasing to the value necessary to overcome the electric strength of the air. If this interval is very short then the successive discharges will produce the same effect as a continuous current through the tube. The consideration of this case may also throw some light on the mechanism by which the discharge is effected, for there are many reasons for believing that in this case the discharge is accomplished by the decomposition of the molecules of the gas, the energy required for this decomposition coming from the electric field, and the consequent exhaustion of the electric energy producing the electric discharge. The reasons which lead us to this conclusion are as follows :

(I) Different gases differ much more in their electric 
strengths than they do in other physical qualities, the difference is much more comparable with the differences between their chemical properties than their physical ones, and the difference between a chemical and a physical process seems to be that in the chemical process the molecules are split up while in the physical one they are not.

(2) In many cases there is direct evidence from both spectroscopic and chemical analysis that this decomposition takes place, and again gases of complex composition whose molecules are easily split up are also electrically very weak.

(3) We can explain by this hypothesis in a general way (Proc. Camb. Phil. Soc. v. 40o) why the electric strength should gradually diminish as the gas gets rarer and rarer, until when the pressure is about that due to a millimetre of mercury the electric strength is a minimum, when the pressure falls below this value the electric strength increases again until at the highest exhaustion which can be got by the best modern air pumps the strength is so great that it is almost impossible to get a spark through the gas.

(4) Dr Schuster has shown (Proc. Royal Society, xxxvir. p. 3 I8) that the electrical discharge through mercury vapour which is supposed to be a monatomic gas presents a peculiar appearance and passes with great difficulty, and quite recently Hertz (Wied. Ann. xxxı. p. 983, 1887) has shown that the electric discharge passes more easily through a gas when it is exposed to the action of violet or ultra-violet light than when it is in the dark; since ultra-violet light has a strong tendency to decompose the molecules of a gas through which it is passing, this is very strong evidence in favour of the view that the discharge is caused by the splitting up of the molecules of the gas.

In the case of the electric discharge through gases the 
insulation seems to be perfect until the electromotive force reaches a definite value, when a spark passes. Thus the field can apparently not be discharged by a rearrangement of the molecules unaccompanied by decomposition. There is evidence however that when the molecules are split up into constituents a state of molecular structure is produced in which the discharge may be produced by rearrangement without further decomposition. Thus Dr Schuster has shown (Proc. Roy. Soc. XLII. p. 37I) that when a strung electric discharge passes through a gas, a very small electromotive force is sufficient to produce a current in a region of the gas screened off from the electrical influence of the primary discharge. Again Hittorf found that a gas was weakened for discharges in the horizontal direction by passing a vertical discharge through it. The diminution in the electric strength of a gas after the passage of a spark can be accounted for in the same way. Again in Mr Varley's experiments on the electric discharge through gases (Proc. Roy. Soc. xix. 236) the quantity of electricity which passed through a tube filled with gas was proportional to $E-E_{0}$ where $E$ is the difference between the potentials of the electrodes and $E_{0}$ a constant electromotive force, in other words the quantity of electricity which flowed through the tube was proportional to the excess of the electromotive force above that which broke the dielectric down; this seems to indicate that the electromotive force $E_{0}$ produces a supply of atoms in the nascent condition and that the rearrangement of these atoms discharges the field.

In the case of fluid insulators the insulation for low electromotive forces is not as in the case of gases perfect. A condenser the plates of which are separated by a liquid dielectric always leaks however small the difference between the potentials of the plates may be. Some experiments 
recently made by Mr Newall and myself (Proc. Roy. Soc. XLII. p. 4IO) showed that for small electromotive forces the leakage obeyed Ohm's law, that is, was proportional to the difference of potential between the plates. This indicates that the leakage is produced by the rearrangement under the electromotive force of some molecular condition, and that this condition is not produced by the electric field, for if it were the leakage would vary as a higher power than the first of the electromotive force. Quincke, who investigated the passage of electricity through the same liquids, using however electromotive forces comparable with those which would produce sparks through the dielectric, found that under these circumstances the quantity of electricity passing through the dielectric varied as a higher power than the first of the electromotive forces, which is just what we should have expected if the electric field split up the molecules of the fluid.

There are many liquids which, though they only conduct electricity with great difficulty when pure, yet when salts or other substances (which may themselves be non-conductors) are dissolved in them, conduct readily. This kind of conduction is called electrolytic and is accompanied by effects which are not observed in other cases.

Since the solvent is not a conductor, the discharge of the electric field which constitutes conduction must in some way or other be due to the action of the substance dissolved in it. The consideration of the discharge through gases as well as the chemical decomposition which always accompanies this kind of conduction suggests that in this case the discharge is caused either by the splitting up of the molecules of the salt by the electric field, or else by the rearrangement when in a nascent condition of the atoms of a molecule of the salt or the constituents of a more complex molecule containing both salt and solvent, the splitting up of 
the molecule being done independently of the electric field. The first of these methods is unlikely for the following reasons.

(I) If it were true it would require a finite electromotive force to start a current through an electrolyte, just as to send a spark through a gas, whilst from the evidence of many experiments it seems clear that the smallest electromotive force is sufficient to start a current through an electrolyte.

(2) The experiments of Prof. Fitzgerald and $\mathrm{Mr}$ Trouton (Report of the British Association Committee on Electrolysis, I886, p. 312) have shown that Ohm's Law is obeyed with great exactness by a current flowing through an electrolyte, whereas if the electromotive force had to break up the molecules the current would be proportional to a higher power than the first of the electromotive force.

(3) If the molecules were split up by the current then the salt will form a greater number of individual systems when the current is flowing than when it is not. Now the rise of the solution in an osmometer and the lowering of its vapour pressure depend upon the number of molecules in unit volume of the liquid and not upon their kind, so that if the number of separate systems is increased by the passage of the current these effects ought to be increased by the passage of a current through the solution. I have lately made some experiments on both these effects and have not been able to detect that the slightest change was made by the current.

For these reasons we conclude that the splitting up of the molecules which allows the current to pass is not caused by the electromotive force but takes place quite independently of the electric field.

The forces between the atoms in a molecule are usually too strong to allow of any arrangement under the electric field, but when the molecule breaks up and these interatomic 
forces either vanish or become very small the constituents of the molecule are free to move under the electromotive force, and they will move so as to diminish the strength of the electric field. In order to form a definite idea of the way in which the field gets discharged we may take the usual view that the constituents into which the molecule splits up are charged with opposite kinds of electricity, and that when the molecule splits up the positively charged constituent travels in one direction, the negatively charged one in the other; in this way we get two layers of positive and negative electricity formed, the electric force due to which neutralizes in the region between the layers the external electric force. The positively charged molecules soon come into the neighbourhood of some negatively charged ones travelling in the opposite direction and they recombine, while the negatively charged ones do the same with some positive molecules, thus the force due to the layers vanishes and the external electric field is re-established to be soon demolished again by the decomposition and rearrangement of other molecules.

Although we suppose that the current is transmitted by the molecules of the electrolyte breaking up, this does not necessarily imply that the electrolyte should when free from electromotive force be largely dissociated, for all that is necessary on this view for the passage of a current is that the molecules of the electrolyte should split up, and there is nothing to prevent our supposing, if other reasons render it probable, that they would instantly re-unite if no electromotive force acted upon them. And since the state of dissociation depends upon the ratio of the time the atoms remain dissociated to the time during which they are combined, we may make this as small as we please and yet have continual splitting up of the molecules. 
There does not seem any necessity for supposing that the passage of electricity through metals and alloys is accomplished in a fundamentally different way from that through gases and electrolytes. For the chief differences between conduction through metals and through electrolytes are (I) that in electrolytic conduction the components of the electrolyte appear at the electrodes, and we have polarization, and (2) that the conductivities of electrolytes increase while those of metals diminish as the temperature increases.

Let us begin by considering the first of these differences, that of polarization. A little consideration will show that we could hardly expect to detect it in the case of metals or alloys, for here instead of, as in electrolytes, the property of splitting up being confined to a few molecules sparsely scattered through a non-conducting solvent, the whole of the molecules can split up, thus the rate of disappearance of any abnormal condition would be almost infinitely greater than in the case of electrolytes, so that if any polarization were produced it would probably die away before it could be detected. Let us next consider the appearance of the constituents of the conductor at the electrodes. The only case in which we could expect to detect this is that of the alloys, but even in this case Prof. Roberts-Austen was unable to detect any change of composition in the alloy round the electrodes; we nuust remember however that an alloy differs very materially from an electrolyte because while in the latter we have a few "active" molecules embedded in a non-conductor, in the former it is as if the solvent as well as the salt conducted, so that the discharge is not concentrated on a few molecules of definite composition but can travel by an almost infinite variety of paths. 
Then again the statements about the effect of heat on the conductivity of elements and electrolytes though true in general are subject to exceptions, thus the conductivities of selenium, phosphorus and carbon increase as the temperature increases; that of bismuth is said to increase at certain temperatures, and I have lately found that the conductivity of an amalgam containing about $3 \circ$ per cent. of zinc and 70 of mercury is greater at $80^{\circ} \mathrm{C}$. than at $15^{\circ} \mathrm{C}$. We must remember too that the rate of increase of conductivity with temperature for electrolytes diminishes as the concentration increases. No sharp line of demarcation can therefore be drawn between the two classes of conductors on this account.

There does not seem any difference between metallic and electrolytic conduction which could not be attributed to the vastly greater number of molecules taking part in metallic conduction, whilst assuming that in all cases the current consists of a series of intermittent discharges caused by the rearrangement of the constituents of molecular systems.

We shall therefore proceed to examine the dynamical results to which such a conception of the electric current leads.

Let us consider the case of an electric field where the electromotive force is everywhere parallel to the axis of $x$. Let the electric displacement in this direction be $f$, then in the Lagrangian function of unit volume of the medium there is the term

$$
-\frac{2 \pi f^{y}}{K}
$$

where $K$ is the specific inductive capacity of the medium. 
This term gives rise to the force

$$
-\frac{4 \pi f}{K}
$$

parallel to the axis of $x$. In consequence of the continual rearrangement of the molecular systems $f / K$ is not uniform but keeps alternately vanishing and rising to a maximum value. If these alterations are sufficiently rapid the effect represented by this term will be the same as that of a steady force equal to its mean value, that is to

$$
-\int_{0}^{1} \frac{4 \pi f}{K} d t \text {. }
$$

Let us suppose that in consequence of the rearrangement of molecular systems $f$ vanishes $n$ times a second, and that $\tau$ is the period which elapses between the end of one period of extinction and the end of the next, then

$$
\int_{0}^{1} \frac{4 \pi f}{K} d t=4 \pi n \int_{0}^{\tau} \frac{f}{K} d t=\frac{n 4 \pi}{K} \beta \mathfrak{f}_{\tau},
$$

where $\mathbf{f}$ is the maximum value of $f$, and $\beta$ a quantity which depends upon the ratio of the time the field is destroyed to that during which it exists.

When the molecular systems rearrange themselves so as to discharge the electric field molecules charged with $f$ units of electricity pass through unit area in one direction, while $\mathbf{f}$ units of negative electricity are carried by molecules moving in the opposite direction.

Thus $2 n f$ is the sum of the positive electricity moving in one direction and of the negative in the opposite passing through unit area in unit time, it is therefore equal to $u$ where $u$ is the intensity of the current, and since $n \tau$ is equal to unity, the force we are considering equals

$$
-\frac{2 \pi \beta}{n K} u
$$


so that this continual breaking down of the field produces the same effect as if the substance possessed the specific resistance $2 \pi \beta / n K$. Thus the greater the number of times per second the displacement breaks down \&c., the better the conductivity.

Now the breaking down of the displacement is caused by the rearrangement of the molecules, and the rearrangement of the molecules in a solid will produce much the same effects as the collisions between the molecules of a gas, and will tend to equalize the condition of the solid, thus we might expect the rate of equalization of temperature to increase with the number of molecular rearrangements. The electrical conductivity would also increase in the same way, so that this view fits in with the correspondence which exists between the orders of the metals when arranged according to thermal and to electrical conductivities.

The preceding investigation of the resistance of such a medium is only valid when the electromotive force is approximately constant over a time which includes a great many discharges. If the displacement were to be reversed during the interval between two successive rearrangements of the molecules the substance would behave like an insulator and not like a conductor. If $\sigma$ is the specific resistance of the substance then

$$
\frac{2 \pi \beta}{n K}=\sigma,
$$

or

$$
n=\frac{2 \pi \beta}{\sigma K},
$$

where all we know about $\beta$ is that it cannot be greater than unity. To find a superior limit to $n$ let us assume that $\beta$ has its maximum value, and that $K$ is $7 / 9 \times 10^{20}$ which is about the same as for light flint glass, then the 
number of times the field breaks down a second is given by the following table:

\begin{tabular}{|c|c|c|c|}
\hline & $\sigma$ & & $n$ \\
\hline Silver & $1 \cdot 6 \times 10^{3}$ & 5 & $\times 10^{17}$ \\
\hline Copper & $1.6 \times 10^{3}$ & 5 & $\times 10^{17}$ \\
\hline Gold & $2.1 \times 10^{3}$ & 4 & $\times 10^{17}$ \\
\hline Platinum & $9 \times 10^{3}$ & 9 & $\times 10^{16}$ \\
\hline Lead & $2 \times 10^{4}$ & 4 & $\times 10^{16}$ \\
\hline Mercury & $9.6 \times 10^{4}$ & 8 & $\times 10^{15}$. \\
\hline $\begin{array}{l}\text { Water with } 8 \cdot 3 \text { per cent. of } \\
\text { sulphuric acid }\end{array}$ & $3.3 \times 10^{9}$ & & $\times 10^{11}$ \\
\hline $\begin{array}{l}\text { Copper sulphate and water } \\
\left(\mathrm{CuSO}_{4}+45 \mathrm{H}_{2} \mathrm{O}\right)\end{array}$ & $10 \times 10^{10}$ & 4 & $\times 10^{10}$ \\
\hline
\end{tabular}

According to the electromagnetic theory of light the electric displacements which constitute light are reversed nearly $10^{15}$ times per second; comparing this with the number of times the field is discharged in an electrolyte, we see that the displacement would be reversed many times a second before it was discharged and hence that such substances would behave like insulators to these rapidly alternating displacements, and so according to the electromagnetic theory of light should be transparent, which as a matter of fact most of them are. Again, we have certainly overestimated $\beta$ and probably underestimated $K$; if we take this into consideration we may conclude that the number of times the field is discharged is probably even in the best metallic conductors not much greater than the number of times the displacements accompanying the propagation 
of light are reversed, hence we need not be surprised that metals in thin films possess a transparency almost infinitely greater than that calculated on the assumption. that their conductivity is the same as that for steady currents.

The number of times the field is discharged at any point will depend upon the number of molecules which split up in unit time and the distance which these travel before combining. If $m$ is the number of times the molecules in unit volume split up in unit time and if it requires $q$ molecules per unit area to be split up in order to discharge the field, then if the molecules after being split up travel a distance $x$ under the influence of the electromotive force before again entering into combination, we shall have

$$
n=\frac{m}{q} x,
$$

since any $q$ molecules which break up within a distance $x / 2$ on either side will discharge the field. Since both $x$ and $q$ will be directly proportional to the electromotive force, $n$ will be independent of it, if the splitting up of the molecules is accomplished by other means.

Since

$$
u=2 n \mathfrak{f}=2 \frac{m}{q} x \mathbf{f},
$$

and since, if the substance is an electrolyte,

$$
\mathfrak{f}=q \boldsymbol{\epsilon},
$$

where $\epsilon$ is the charge on either of the ions into which the molecule splits up, we have

$$
u=2 m x \epsilon .
$$

So that if $N$ be the number of molecules of the salt in unit volume

$$
\frac{u}{2 N \epsilon}=\frac{m}{N} \cdot x
$$


$=x \times$ (number of times each molecule breaks up per second) $=$ the distance between the two ions at the end of one second.

- But $u / 2 N \epsilon$ is, see Lodge, Report on Electrolysis, British Association Report, 1885, p. 755, the quantity called by Kohlrausch the sum of the velocities of the ions, and if we assume that the ratio of the velocities is given by experiments on the migration of the ions, this view of the current would lead to the same expression for the absolute distance travelled by each ion in unit time as that given by Kohlrausch.

A full discussion of this would however lead us too far from our purpose, which is merely to use this conception of a current to deduce reciprocal relations from the effects of various physical agencies on resistance.

The specific resistance of a substance according to our view is

$$
\frac{2 \pi \beta}{n K},
$$

and if this varies when the circumstances are changed it may be because either $\beta$, $n$, or $K$ are changed. To take an example the resistance of a metal wire seems to be slightly affected by strain, this may arise either from the specific inductive capacity being altered by strain, or by the strain altering the number of times a second the molecules split up, or finally by an alteration in the time the field remains discharged. The term

$$
-\frac{2 \pi f^{2}}{K}
$$

in the Lagrangian function corresponds, see $\S 35$, to a force equal to

$$
2 \pi f^{2} \frac{d}{d e} \frac{\mathbf{I}}{K}
$$


tending to produce an extension $e$. Thus unless the alteration in the resistance was due to the alteration of $K$ with the strain there would be no corresponding elastic force. If however it does arise from the alteration of $K$ with the strain the mean value of the elastic force is

$$
\begin{array}{r}
2 \pi \frac{d}{d e} \frac{\mathrm{I}}{K} \int_{0}^{x} f^{2} d t, \\
\int_{0}^{x} f^{2} d t=\alpha \mathbf{f}^{2}=\alpha \frac{u^{2}}{n^{2}},
\end{array}
$$

and

where $a$ is a number which cannot be greater than unity, and which like $\beta$ depends upon the time the field remains discharged.

Thus the mean value of the elastic force

$$
\begin{aligned}
& =-\frac{2 \pi u^{2} \alpha}{K n^{2}} \cdot \frac{d \log K}{d e} \\
& =-\frac{\sigma u^{2} a}{\beta n} \frac{d \log K}{d e} .
\end{aligned}
$$

For good conductors this term will be exceedingly small on account of the smallness of $\sigma / n$, see the table p. 300 , and even for bad conductors it will never get large enough to make it comparable with the large forces required to produce an appreciable change in the extension.

If $x$ is a coordinate of any type this term indicates a force of type $x$ equal to

$$
\frac{\sigma}{\beta} \frac{a u^{2}}{n} \frac{d \log K}{d x},
$$

or as it may be written

$$
=\frac{\sigma^{2} u^{2} \alpha K}{2 \pi \beta^{2}} \frac{d \log K}{d x} .
$$

Now $K$ is of the order $10^{-21}$ and $\sigma u$, the electromotive 
force, even for a fall of ro volts per centimetre, is only $10^{9}$, so that in this case the force of type $x$ is of the order

$$
10^{-3} \frac{\alpha}{2 \pi \beta^{2}} \frac{d \log K}{d x},
$$

and so is exceedingly small; hence we conclude that the reciprocal effects corresponding to the effects observed on the resistances are probably much too small to be capable of detection unless for very bad conductors under the influence of electromotive forces comparable with those used in experiments on static electricity. 


\section{INDEX.}

Absolute temperature, 95

Absorbed air, effect of on vapour pressure, $\mathbf{I} 73$

Absorption of gases by liquids, 179

Action, Least, 14

Adie, membranes for osmometers, 186

Affinity, determination of coefficient of, by the observation of the - electromotive force of a galvanic cell, 271

Alloys, electric resistance of, $29^{6}$

Battery, electromotive force of, $26_{5}$

- Gas, 275

Baur, effect of temperature on magnetization, I05

Berthelot, Law of Maximum work, 220

Bertrand, vapour pressure, $16 \mathbf{I}$

Bevan, Young's modulus for ice, 259

Bidwell, effect of magnetization on the length of an iron bar, 54

Boltzmann, residual torsion, 130

Bosscha, forms of clouds, 203

Bunsen, absorption of gases by liquids, 181

Capillarity, effect of, on electromotive force required to decompose an electrolyte, 87

effect of, on vapour pressure, $16_{2}-16_{3}$

effect of, on solubility, $251-255$

effect of, on freezing point, 262

effect of, on density of salt solutions, $191-192$

effect of, on dissociation of gases, 203

effect of, on chemical equilibrium, $234-237$

Cassie, effect of temperature on specific inductive capacity, $6_{5}, 102$

Chemical equilibrium, 215

$$
\text { effect of pressure on, } 221,237
$$

effect of surface tension on, $234-237$

effect of magnetization on, $24^{\circ}$

Clirystal, article on electricity quoted, 136 effect of temperature on, 221

\section{magnetism quoted, $6 \mathbf{1}$}

Circularly polarized light, magnetic effects produced by, 78

Clausius, I 43

-_- expression for force between two moving electrified spheres, $3^{6}$

- - formula for an imperfect gas, 197

T. D. 
Coefficient of magnetization, effect of temperature on, 105

Coefficient of self induction, 40

Compressibility of salt solutions, 183

Constitution of bodies, Maxwell on, 133-134

Controllable coordinate defined, 94

Coordinates, definition of, is

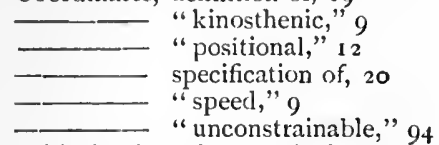

Critical value of magnetic force, $5^{6}$

Currents, induction of, $4 \mathrm{I}$

- mechanical force between, 4o

- effect of, on elasticity of wires, 43

Czapski, variation of electromotive force with temperature, 99

Dielectric, effect of moving conductors on stiffness of, $3^{8}$

strain in due to electrification, 62

Diffusion of salts, 182

Discharge, electric, through gases, 29 !

Discrough liquids, 293

Discharges, number of, in electric field, 300

Dissociation, 193

of nitrogen tetroxide, 200

- of phosphorus pentachloride, 208

Boltzmann on, 200

Willard Gibbs on, 200

effect of electricity on, 207

effect of presence of neutral gas on, 207

effect of surface tension on, $2 \mathrm{O}_{4}$

effect of pressure on, $22 \mathrm{I}$

effect of temperature on, 201

- of a solid into two gases, 210

of salts in solution, 2 I 2

Dissolved salt, effect on vapour pressure, 175

Dupré, on vapour pressure, $16 \mathrm{I}$

Dynamical interpretation of temperature, 90

Elasticity of a wire, effect of current on, 43

Electric resistance, 289

Electric discharge through gases, 291

Electricity, inertia of, $3^{2}$ through liquids, 293

—_ specific heat of, ro6

-__ effect of, on vapour pressure, $16_{4}-16_{7}$

- effect on dissociation, 207

thermal effects produced by charge of, 1 IO- $I_{12}$

Electrification, strain in a dielectric due to, $6_{2}$ thermal effects due to change of, 102 
Electrolyte, effect of pressure on electromotive force required to decompose, $83-85$

capillarity on electromotive force required to decompose, 87

Electrolytic conduction, 293

Electromotive force due to variation of the magnetic field, 68

produced by twisting a maguetized wire, $7 \mathrm{I}$

required to decompose an electrolyte, effect of pressure on, $84,85,87$

required to decompose an electrolyte, effect of capillarity on, 87

produced by inequalities in temperature, I 66

of batteries, 265

Energy, "free," 95

of gas batteries (table of), 275

Entropy, 157

Equilibrium, chemical, 2 I 5

- effect of strain on, 239

- $\begin{aligned} & \text { effect of pressure on, } 22 \mathrm{r}, 238 \\ & \text { effect of surface tension on, 234-237 }\end{aligned}$

- - effect of magnetization on, $24^{\circ}$

effect of temperature on, 22 I

v. Ettinghausen and Nernst, effect produced by flow of heat in a magnetic field, i 6

Evaporation, ${ }_{15} 8$

- effect of strain on, $\mathbf{r} 68$

- effect of pressure on, $168-172$
effect of surface tension on, $16_{2}$
effect of electrification on, $16_{4}$

Ewing, effect of strain on magnetization, 55

- critical value of magnetic force, ${ }_{5} 6$

- hysteresis, ${ }^{0} 4$

Faraday, force on soft iron in magnetic field, 47

Fitzgerald, on Hall's phenomenon, 73

- on Ohm's law in electrolytic conduction, 294

Force on a body in a magnetic field, 47

- a conductor carrying a current, 69

- magnetic, due to currents, 68

- electromotive, due to variation in magnetic field, 68

Free energy, 95

Freezing point, effect of pressure on, 257-259

- effect of torsion on, 260

effect of surface tension on, 262

effect of dissolved salt on, 262

Gas, Lagrangian function for a perfect gas, I 54

Gas batteries, 265

- _ Pierce's determination of electromotive force of, 275

- with one gas, 276

Gases, absorption of, by liquids, I 79 
Gases, electric discharge through, 291

Gibbs, Willard, on dissociation, 200

on the electromotive force of batteries, 277

Glazebrook, on Hall's phenomenon, 73

Groves gas batteries, 266

Guldberg and Waage, on chemical equilibrium, 225

Hall's phenomenon, 72

Hamilton, principle of varying action, 9

Height to which a salt solution rises in an osmometer, 188

v. Helmholtz, conservation of energy, 2

- objection to Weber's law of force between two elcctrified spheres, 37

__ "freie energie," 95

variation of electromotive force with temperature, 99,269

strain produced by a magnetic field, $5^{2}$

Hertz, action of light on the electric discharge, 29I

Hittorf, discharge of electricity through gases, 292

Hoff, van $t^{\prime}$, pressure in dilute solutions, I 75

osmotic pressure, 188

effect of walls of vessel on chemical action inside, 206

Hopkinson, J., residual charge of a Leyden jar, 136

Horstmann, chemical equilibrium, 229

Hysteresis, 104

Induction of electric currents, $4^{\mathrm{I}}$

Inertia of electricity, $3^{2}$

Irreversible effects, 28 I

Jahn, variation of electromotive force with temperature, 99

Joule, elongation of a bar produced by magnetization, 54,59

- effect of magnetization on the volume of a magnet, $5^{6}$

Kinosthenic coordinates defined, 9

Kirchhoff, strain produced by a magnetic field, 52, 54

Kohlrausch, electrolytic conduction, 302

Lagrangian function, expressions for, 23,29

mean value of stationary, $145,146,147$

expression of a gas, 152

Larmor on varying action, 18

Law, second, of Thermodynamics, 99

Least action, 14

Leyden jar, residual charge of, I 36

Liebreich, inert space in chemical reactions, $23^{6}$

Light, magnetic force produced by, 78

Liquefaction, 255

Liquids, discharge of electricity through, 293

Liveing, effect of surface tension on chemical action, 237 
Lodge, Report on Electrolysis, 302

Magnetic field, stress produced by, 47

- force due to currents, 68

- produced by twisting a wire conveying a current, $7^{2}$

inertia, $6_{5}$

arising from the Hall effect, 74

Magnetism, Ewing's researches on, 53, 54

Magnetization, terms in Lagrangian function depending on, 44

effect of torsion on, $6 \mathbf{I}$

due to torsion, $6 \mathrm{I}$

strains produced by, $50-58$

change of length due to, 54

effect of strain on, $50-58$

thermal effects due to, 103

effect on chemical action, 240

Mass, effects due to, in chemical equilibrium, 223

Maximum work, Berthelot's Law of, 220

Maxwell, Electricity and Magnetism, 41, 44, 45, 47, 49, 67, 289

- Theory of Heat, $81,90,98,164$

- strains produced by an electric field, 64

a magnetic field, 52

on the constitution of bodies, $\mathrm{I}_{33}, \mathrm{I}_{34}$

Mechanical force between circuits conveying currents, to

Mercury vapour, electric discharge through, 29 I

Meyer, Lothar, Modernen Theorien der Chemie, 225

Meyer, Victor, effects of surface of 'vessel on dissociation, 207

Monckmann, effect of surface tension on the density of salt solutions, $19^{2}$

Muir's Principles of Chemistry, 2 1 2, 225

Natanson, E. and L., dissociation of nitrogen tetroxide, 200

Neesen on residual torsion, 130

Nernst and v. Ettinghausen, electromotive forces due to flow of heat in a magnetic field, 116

Neutral gas, effect of, on the dissociation of a solid into two gases, 212

Newall, discharge of èlectricity through liquids, 293

Ohm's Law for electrolytes, $29+$

Osmometer, 186

Osmosis, 186

Peltier effect, II 5

Pfeffer on osmotic pressure, 175

Pfeffer's Osmotische Untersuchungen, 186

Phosphorus pentachloride, dissociation of, 208

Pierce, electromotive force of gas batteries, 275

Polarization, rotation of plane of, by magnetic field, 78

"Positional " coordinates, I 2

Potential energy, I 4 
Pressure, effect of, on chemical equilibrium, 22 I, 237 solubility, $245-25 \mathrm{I}$ the electromotive force required to decompose an electrolyte, $84-85$ of gas batteries, 273

the freezing point of liquids, 257 evaporation, $168-172$

osmotic, I 75, I 88

Quincke, discharge of electricity through liquids, 293

strains in a dielectric due to electrification, 64

Rain drops, effect of pressure on the formation of, $\mathbf{1 7 2}$

Raoult, effect of dissolved salt on vapour pressure, 178 the freezing point of solutions, 175,263

Rayleigh, Lord, coefficient of magnetization for small magnetic forces, 46 reciprocal relations, 8 I Theory of Sound, 8.

Reciprocal relations, $8 \mathbf{I}$

Residual charge of a Leyden jar, 136

Residual effects, 128

Resistance, electrical, $\mathbf{2 8 9}$

effect of strain on, 302

Reversible thermal effects due to a current of electricity, ro9, 269

Riemann, law of force between two moving electrified spheres, 37

Roberts-Austen, conduction through alloys. 296

Röntgen and Schneider on the compressibility of salt solutions, 83,183

Routh, Rigid Dynamics, 9 surface tension of salt solutions, 87,254

- Stability of Motion, Io

Rowland, rotation of plane of polarization of light in a magnetic field, 79

Salt solutions, compressibility of, $8_{3}, 183$

Salts, diffusion of, 182 surface tension of, 87,254

- dissociation of, in solutions, 212

- effect of, on freezing point, 262

- effect of pressure on the solubility of, $245-251$

Schneider, see Röntgen and Schneider.

Schumann, compressibility of salt solutions, 183

Schuster, electric discharge through mercury vapour, 29 I

Second Law of Thermodynamics, 99 gases, 292

Self induction, coefficient of, to

Soluhility, effect of pressure on, $245-25 \mathrm{I}$ of liquids in fine drops, $25^{2}$

Solutions, compressibility of, 183 pores, 255 
Solutions, dissociation of salts in, 212

Sorby, effect of pressure on solubility, 247

Specific heat of electricity, 106

Specific inductive capacity, effect of temperature on, $6_{5}, 102$

Spheres, force between two moving electrified spheres, 35

Steady state, 8o

Strain in a dielectric due to electrification, 62

- effeci on vapour pressure, 168

chemical equilibrium, 239

- freezing point, 259

- solubility, $25 \mathrm{I}$

electric resistance, $3^{\circ 2}$

change of temperature due to, Ior

thermoelectric effects of, i i 3

Streintz, effect of current on elasticity of wire, 43

Stresses produced by magnetic field, 47

Surface tension of solutions, $19^{\circ}$

Surface tension, effect of on the electromotive force required to decompose an electrolyte, 87

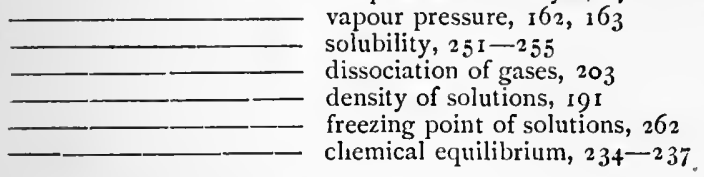

Tait, Thomson and, Natural Philosophy, 18

Temperature, absolute, 95

effect on specific inductive capacity, 65,102

- _ electromotive force of batteries, 99 coefficient of magnetization, 105

chemical equilibrium, 22 I

dissociation, 201

change of, produced by strain, 101

Thermal effects due to change of electrification, 102

- magnetization, 103

change of strain, io

reversible, due to a current of electricity, 109

produced by communicating a charge of electricity to a body, $110-112$

Thermodynamics, Second Law of, 99

Thermoelectric effects of strain, 113

Thermoelectricity, $110-127$

Thomsen, Thermochemische Untersuchungen, 227

Thomson, James, effect of pressure on the freezing point of water, 257

Thomson, Sir William, effect of strain on magnetization, 48,52

- change of temperature due to strain, 101 
Thomson, Sir William, effect of pressure on the freezing point of water, 257

thermoelectric effect due to inequalities of temperature in a conductor, 106

Thomson and Tait, Natural Philosophy, I 8

Tomlinson, effect of a current on the elasticity of wire, 43

Torsion, in a magnetized wire due to current, $7 \circ$

- produced by magnetization, $6 \mathbf{I}$

- effect on magnetization, $6 \mathbf{r}$

- effect on freezing point, 260

Torsional viscosity, 139,287

Trouton, Ohm's Law in electrolytes, 294

Unconstrainable coordinate, defined, 94

Vapour pressure, $\mathbf{I} 6 \mathbf{r}$

- effect of surface tension on, I $63, \mathrm{r} 6_{4}$

- effect of electrification on, 165,166

- effect of strain on, 168

- - effect of pressure on, $168-172$

- effect of absorbed air on, 173

Varley, discharge of electricity through gases, 292

Villari, effect of strain on magnetization, $48-5^{2}$

Viscosity, torsional, 287

Waage and Guldberg, on chemical equilibrium, p. 225

Waals, van der, $9 \circ$

_ formula fơr an imperfect gas, 197

surface tension of gases, 204

Weber's expression for the force between two electrified spheres in motion, 36

v. Helmholtz's objection to, $\mathbf{3 7}$

Wiedemann, G., connexion between torsion and magnetization, $6 \mathbf{r}$ torsion produced by a current flowing along a magnetized wire, 70,72

Work, Berthelot's Law of Maximum, 220 


\section{MESSRS MACMILLAN \& CO.'S PUBLICATIONS.}

\section{BY THE SAME AUTHOR.}

The Motion of Vortex Rings, a Treatise on. An Essay to which the Adams Prize was adjudged in 1882 in the University of Cambridge. With Diagrams. 8vo. $6 s$. Nature says:-

"Both as regards the interest of the subject and the treatment it has received at the hands of the author we do not doubt that the essay before us is destined to take a foremost place amongst the essays which have been called forth, or at all events distinguished, by the Adams Prize.... Besides discussing the theory of gases, Mr Thomson goes somewhat fully into a vortex atom theory of chemical combinations; in this he raises many points which will doubtless be of great interest should the hypothesis survive the crucial test by the theory of gases which this essay now for the first time renders possible. Of the mathematical interest of the essay we can only say that to those who can appreciate it this will be found to be very great."

Reprint of Papers on Electrostatics and Magnetism. By Sir William Thomson, D.C.L., LL.D., F.R.S.E., Fellow of St Peter's College, Cambridge, and Professor of Natural Philosophy in the University of Glasgow. Second Edition. 8vo. I $8 s$.

The Mathematical Theory of perfectly Elastic Solids, with a short account of Viscous Fluids. An Elementary Treatise. By William John IbBetson, M.A. 8vo. 2 Is.

Four-Figure Mathematical Tables. Comprising Logarithmic and Trigonometrical Tables, and Tables of Squares, Square Roots, and Reciprocals. By J. T. BotTomley, F.C.S. 8vo. $2 s .6 d$. Physical Arithmetic. By Alexander Macfarlane, M.A., D.Sc., F.R.S.E. Crown 8 vo. 7 s. $6 d$.

A Text-Book of the Principles of Physics. By Alfred DANiell, M.A., LL.B., D.Sc., F.R.S.E. With Illustrations. Second Edition. Revised and Enlarged. Medium 8vo. $21 s$.

Applied Mechanics : an Elementary General Introduction to the Theory of Structures and Machines. By Professor James H. Cotterill, F.R.S. Medium 8vo. i $8 s$.

The Mechanics of Machinery. By A. B. IV. KennedY, F.R.S., M.Inst.C.E. With Illustrations. Crown 8vo. I $2 s .6 d$.

Absolute Measurements in Electricity and Magnetism. By ANDREw GraY, M.A., F.R.S.E. Two Vols. Crown 8vo. Vol. I.

[Immediately.

The Differential Calculus. With Applications and numerous Examples. An Elementary Treatise by Joseph Edwards, M.A. Crown 8vo. ros. 6ul.

Differential and Integral Calculus. With Applications. By A. G. Greenhill, M.A. Crown 8vo. $7 s .6 d$.

Constructive Geometry of Plane Curves. By T. H. EAGLES, M.A. With numerous Examples. Crown 8vo. i2s.

Heat and Electricity, a Collection of Examples on. By H. H. TURner, B.A. Crown 8vo. 2s. $6 d$. 


\section{MESSRS MACMILLAN \& CO.'S PUBLICATIONS.}

BY CHARLES SMITH, M.A.

Elementary Algebra. Globe 8vo. 4s. 6d.

A Treatise on Algebra. Crown 8vo. $7 s .6 d$.

Conic Sections. Fourth Edition. Crown 8vo. 7 s. $6 d$.

An Elementary Treatise on Solid Geometry. Second Edition. Crown 8vo. 9s. 6d.

By Messrs H. S. HALL and S. R. KNIGHT.

Elementary Algebra for Schools. Fourth Edition, Revised and Corrected. Globe 8vo. $3 s .6 d$.; with Answers, $4 s .6 d$.

Algebraical Exercises and Examination Papers. To accompany "Elementary Algebra." 2nd Edition. Globe 8vo. . 2s. 6d. Higher Algebra. A Sequel to "Elementary Algebra for Schools." Second Edition. Crown 8vo. 7s. $6 d$.

By the Rev. J. B. LOCK, M.A.

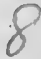

Higher Trigonometry. Fifth Edition. Globe 8vo. 4s. $6 d$. Dynamics for Beginners. Globe 8vo. 3s. $6 d$.

Statics for Beginners. Globe 8vo. [In the press.

Higher Arithmetic and Elementary Mensuration, for the Senior Classes of Schools and Candidates preparing for Public Examinations. By P. Goyen. Globe 8vo. 5s.

Kinematics and Dynamics. An Elementary Treatise. By James Gordon MacGregor, M.A., D. Sc. Crown 8vo. Ios. $6 d$. A Treatise on Elementary Statics. By JoHn Greaves, M.A. Crown 8vo. 6s. 6 d.

A Treatise on Differential Equations. By ANDREw Russell Forsyth, M.A., F.R.S. 8vo. Its.

The Elements of Dynamic. An Introduction to the Study of Motion and Rest in Solid and Fluid Bodies. By W. K. ClifFord, F.R.S., London. Part I. - Kinematic. Crown 8vo. Books I-III. 7s. 6d.; Book IV. And Appendix. 6s.

Introduction to Quaternions, with numerous Examples. By P. Kelland, M.A., F.R.S., and P. G. TAit, M.A. Cr. 8 vo. ${ }^{3}$ s. $6 d$.

A Treatise on the Theory of Determinants. With graduated sets of Examples. For use in Colleges and Schools. By THos. Muir, M.A., F.R.S.E. Crown 8vo. 7s. $6 d$.

By the Rev. John J. Milne, M.A.

Weekly Problem Papers. With Notes intended for the use of Students preparing for Mathematical Scholarships, and for the Junior Members of the Universities who are reading for Mathematical Honours. Pott. 8vo. 4s. $6 d$. Solutions. Crown 8vo. Ios. $6 d$.

Companion to "Weekly Problem Papers." Cr. 8vo. 10s. $6 d$.

MACMILLAN AND CO, LONDON. 


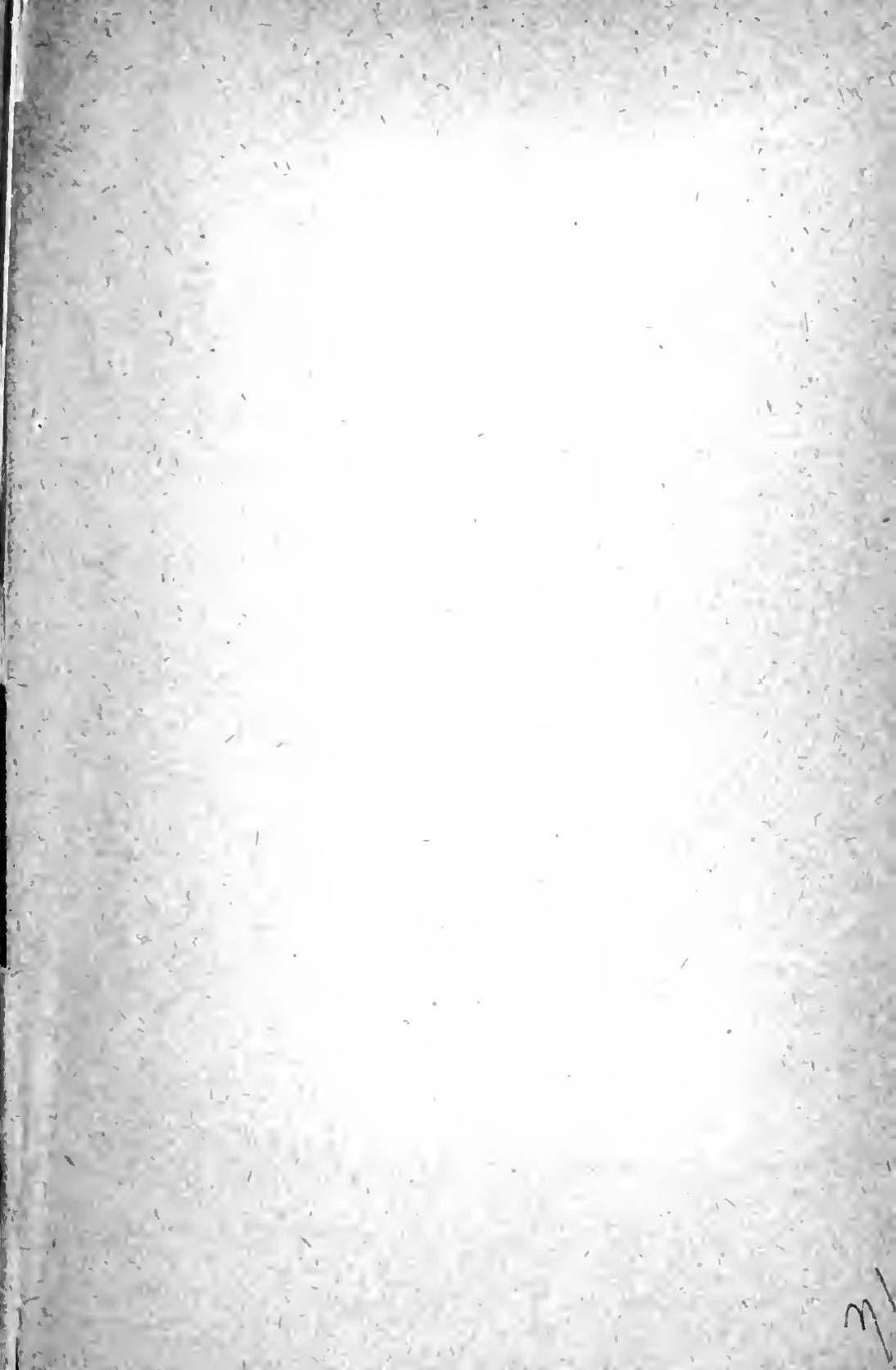





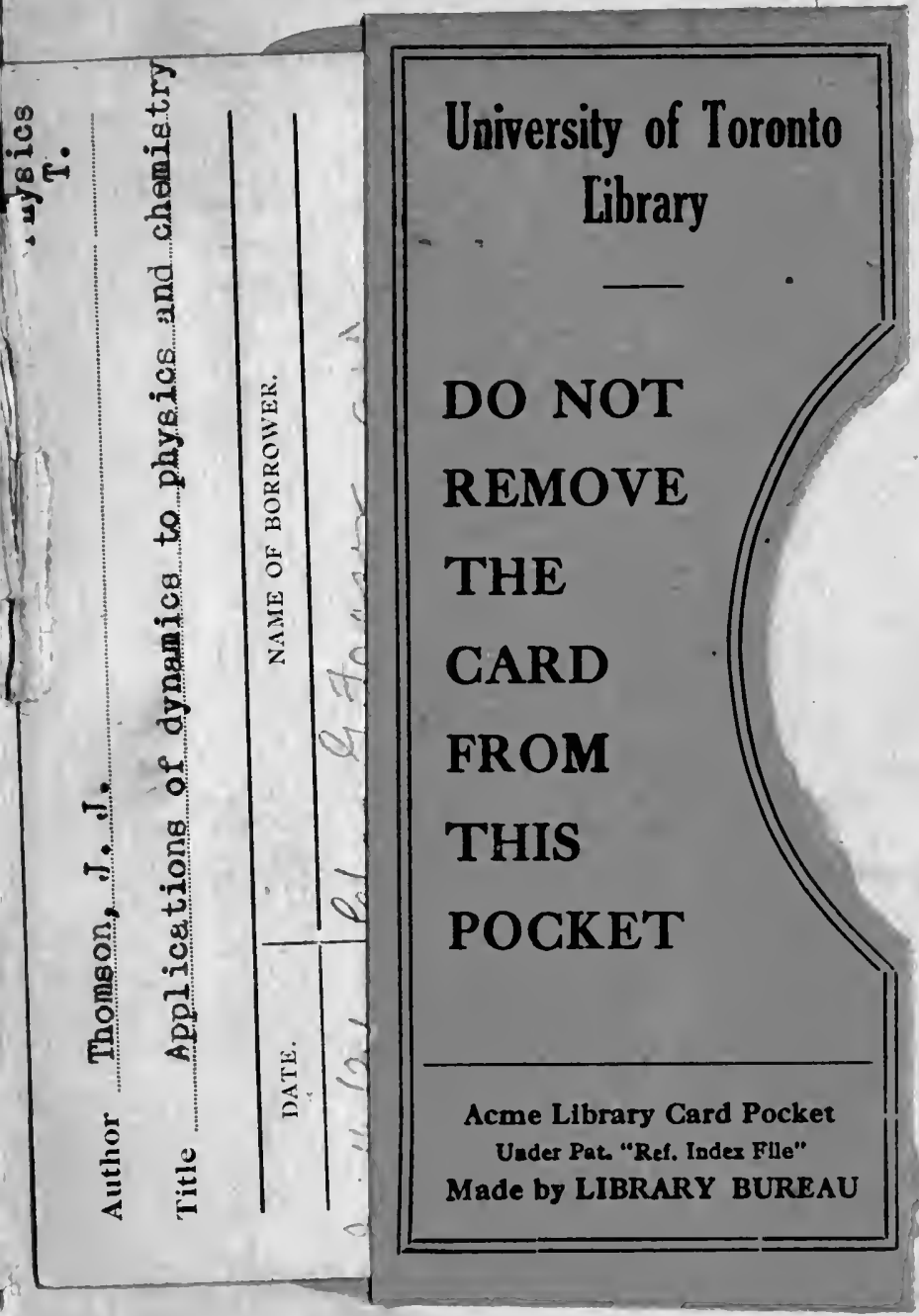




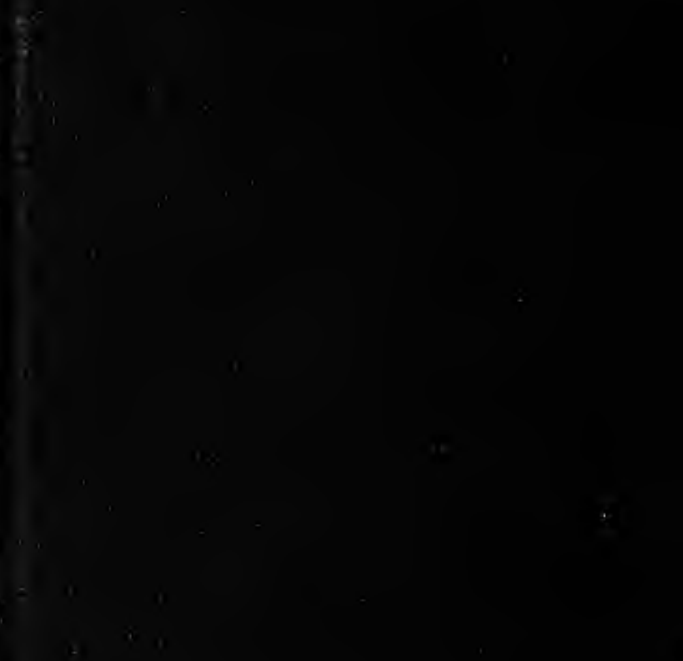

